José Anderson do Nascimento Batista

\title{
Modelagem de Escoamento em Aqüíferos Longos Baseada no Método de Elementos Analíticos
}

\author{
Tese apresentada à Escola de Engenharia de \\ São Carlos como parte dos requisitos para a \\ obtenção do Título de Doutor em Ciências da \\ Engenharia Ambiental. \\ Orientação: Prof. Harry Edmar Schulz \\ Co-orientação: Prof. Edson Cezar Wendland
}

São Carlos - SP 
Ficha catalográfica preparada pela Seção de Tratamento da Informação do Serviço de Biblioteca - EESC/USP

B333m

Batista, José Anderson do Nascimento

Modelagem de escoamento em aqüiferos longos baseada no método de elementos finitos / José Anderson do Nascimento Batista. -- São Carlos, 2006.

Tese (Doutorado) - Escola de Engenharia de São Carlos-Universidade de São Paulo, 2006.

Área: Ciências da Engenharia Ambiental.

Orientador: Prof. Dr. Harry Edmar Schulz.

Co-orientador : Prof. Edson Cezar Wendland.

1. Hidráulica Ambiental. 2. Método de elementos analíticos. 3. Aqüíferos longos. I. Título. 
FOLHA DE JULGAMENTO

Candidato: Engenheiro JOSÉ ANDERSON DO NASCIMENTO BATISTA

Tese defendida e julgada ena $10-02-2006$ perante a Comissão Julgadora:

Prof. Titular HARRY/EDMAR SCHULZ (Orientador)

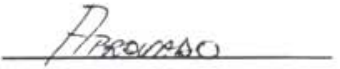

(Escola de Engenharia de São Carlos/USP)

Prof. Associađo FERNANDO MARTINI CATALANO

Apuovado

(Escola de Engenharia de São Carlos/USP)

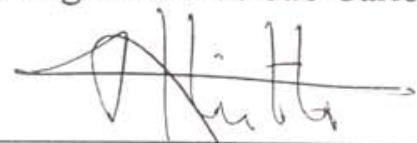

Prof. Associado ANTONIO MAROZZI RIGHETTO

Aproverdo

(Escola de Engenharizd de São Carlds/USP)

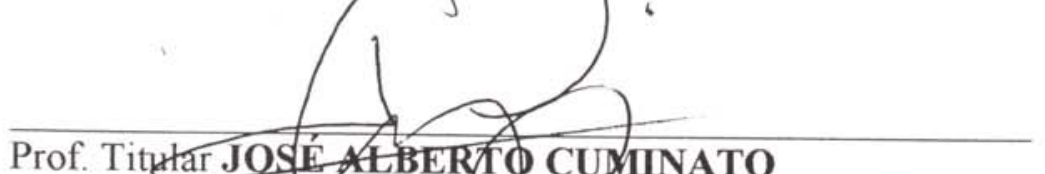

Prof. Tityar JOSE EBERT $\phi$ CUMINATO

(Instituto de Ciêncras Matemáticas e de Computação/USP)

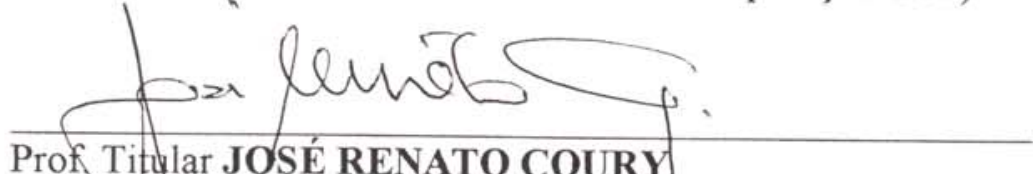

Prof Titular JOSÉ RENATO COURY

APOOVADC

(Universidade Federal de São Carlos/UFSCar)
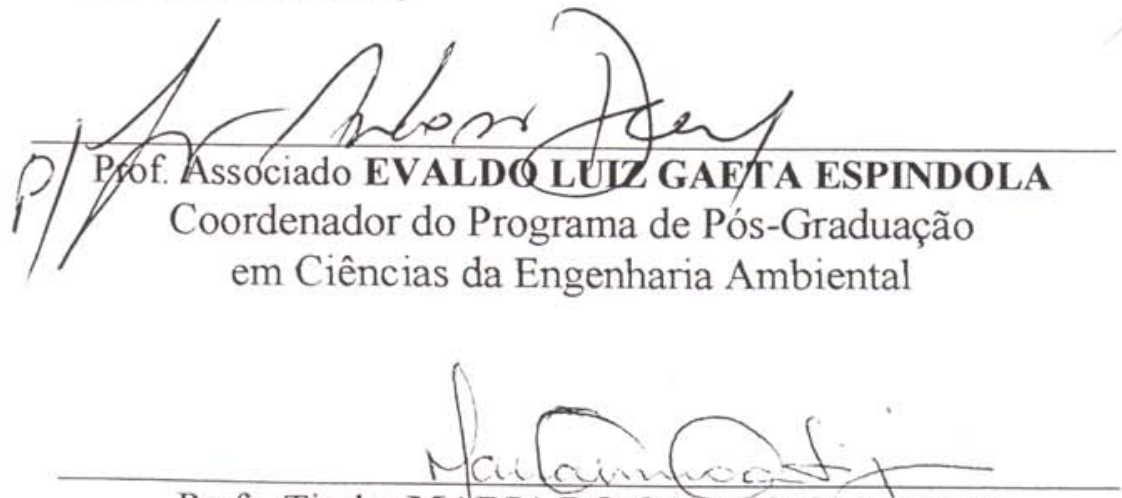

Profa. Titular MARIA DO CARMO CALIJURI

Presidente da Comissão de Pós-Graduação da EESC 
Dedico para

Sabrina, Filipe e Pedro. 


\section{Agradecimentos}

Os agradecimentos a seguir são informais, porém, necessários e existem em função dos inúmeros atos igualmente desobrigados realizados em benefício deste trabalho. Esta tese é fruto de muitas conversas, discussões, sugestões, críticas que produziram esclarecimentos, dúvidas e, principalmente, motivação. Logo no início, colegas de classe com quem conversei, ou apenas comentários de amigos a respeito estimularam-me à curiosidade sobre o tema. Com a chegada dos primeiros resultados, as dúvidas levantadas por pessoas interessadas em palestras que fiz e, de outro lado, correspondências que mantive com diversos autores via email foram necessárias para me alertarem para dificuldades. No entanto, ao longo de todo trabalho, o simples fato de expor um problema a um colega muitas vezes permitiu obter colocações ou questões que valeram de preciosos "feedbacks". Neste sentido, posso dizer que Jorge e Quaresma são as pessoas mais pacientes e solidárias que existem. A caminho do final do trabalho, próximo ao término do prazo, a compreensão do Professor Harry pôs fim a qualquer ansiedade. Tenho também muito a agradecer ao Professor Edson pela confiança que dispensou a mim nos seus projetos de extensão durante o período pós-bolsa. Entretanto, é preciso, ainda, mencionar o fato de que este trabalho não teria sido possível sem a oração de meus pais, minha sogra e minha esposa (Sabrina). Portanto, esta tese é fruto de muita motivação, construída por meio da didática formal, mas, também, da informal e esta regida por Deus. A Ele toda gratidão e louvor. 
"Passa diante do povo, e toma contigo alguns anciãos de Israel, leva contigo em mão a vara, com que feriste o rio, e vai. Eis que estarei ali diante de ti sobre a rocha de Horebe; ferirás a rocha, e dela sairá água, e o povo beberá".

Êxodo 17. 5, 6

"Toma a vara, ajunta o povo, tu e Arão, teu irmão, e, diante dele, falai à rocha, e dará a sua água; asssim lhe tirareis água da rocha, e dareis a beber à congregação e aos seus animais".

Números 20. 8 


\section{Resumo}

Batista, J. A. N. (2006) Modelagem de Escoamento em Aqüíferos Longos Baseada no Método de Elementos Analíticos. Tese de Doutorado. Universidade de São Paulo. São CarlosSP.

O Método de Elementos Analíticos tem se mostrado uma alternativa conveniente para a construção de modelos regionais de escoamento subterrâneo. O Método utiliza soluções elementares analíticas e, portanto, não necessitam de contornos artificiais. Entretanto, a caracterização de fronteiras externas de aqüíferos considerados longos (Aqüíferos Longos) acarreta dúvidas quanto à presença do efeito de borda na simulação. Assim, neste trabalho, o Método de Elementos Analíticos é estendido para a modelagem de escoamentos em Aqüíferos Longos eliminando-se a possibilidade da presença de efeitos de borda. O domínio desses aqüíferos é mapeado em um semi-plano utilizando-se a transformação de SchwarzChristoffel. Dessa forma, elementos de regime estacionário são mapeados juntamente com o domínio para o semi-plano e, portanto, sua formulação é modificada. Os contornos do semiplano, por sua vez, são representados pelo Método de Imagens. Elementos de escoamento transiente são abordados utilizando-se a formulação de Zaadnoordijk. São realizadas correções algébricas e computacionais na formulação desses elementos. No entanto, o mapeamento de domínio não é extensivo aos elementos transientes. Sua aplicação em Aqüíferos Longos é feita de maneira aproximada utilizando-se apenas o Método de Imagens diretamente sobre o aqüífero. Em seguida, a extensão proposta para o Método de Elementos Analíticos é analisada em um problema hipotético. Finalmente, é feita uma modelagem para a avaliação de impactos ambientais devidos a um sistema de captação de água localizado em uma porção do Aqüífero Barreiras no Estado do Rio Grande do Norte sobre a Lagoa do Bonfim. O modelo é calibrado em regime permanente fornecendo uma estimativa de recarga de $24 \mathrm{~mm} / \mathrm{mês}$ e, então, estima os impactos permanentes do sistema sobre a Lagoa do Bonfim, onde é feita a captação. O impacto do bombeamento reduz aproximadamente 5,36m do nível de água natural da Lagoa. Um cenário de seca é, também, simulado em regime transiente. A partir dos resultados obtidos avalia-se que dois meses de ausência de recarga direta aumentam aproximadamente $0,5 \mathrm{~m}$ a redução do nível de água da Lagoa do Bonfim.

Palavras-chaves: Hidráulica Ambiental, Método de Elementos Analíticos, Aqüíferos Longos. 


\begin{abstract}
Batista, J. A. N. (2006) Elonged Aquifer Flow Modeling Based on Analytic Element Method. Doctoral Thesis. Universidade de São Paulo. São Carlos-SP.

The Analytic Element Method has shown itself an elegant alternative to regional groundwater flow modeling. The Method applies elementary analytic solutions and, therefore, artificial boundaries are not a necessity. However, frontiers of such considered Elonged Aquifers carries out some questions about "edging effects" in the model results. In this work, the Analytic Element Method is extended to Elonged Aquifer flow completely eliminating any "edging effect". To do this, such aquifers are mapped into a semi-plan by means of the Schwarz-Christoffel transform. Next, steady flow elements are mapped accordingly to the domain transformation and consequently its formulas are modified suitably. Then, Method of Image gives boundary conditions in semi-plan. Transient flow elements due to Zaadnoordijk are considered. Mistakes in algebraic and computational aspects were corrected. Nevertheless, domain mappings are not performed to transient elements. Appliance of such elements to Elonged Aquifers is performed in an approximated manner with the Method of Image just upon the aquifer. After that, a hypothetic example is performed in order to analyze the new elements, given successful results. Then, a case study is developed in order to assess the impact due to a water supply system in the Barreiras Aquifer, Rio Grande do Norte State, on Bonfim Lake. The model is calibrated, obtaining the recharge rate parameter equal to $24 \mathrm{~mm} / \mathrm{month}$, and then is used to evaluate water level depletion in Bonfim Lake. Water level changing in Bonfim Lake is given by the steady state flow $5.36 \mathrm{~m}$ down. Finally, a transient flow model is built in order to obtain water level changes due to recharge absence. According to results, recharge absence during two month long enlarges $0.5 \mathrm{~m}$, approximately, water depletion in Bonfim Lake.
\end{abstract}

Key-words: Environmental Flow, Analytic Element Method, Elonged Aqüífers 


\section{Lista de Simbolos}

A matriz de coeficientes

A, $B$ constantes determinadas por condições de contorno

B vetor de termos independentes

C contorno de uma superfície de controle; constantes de integração

c vetor de intensidade do dipolo no espaço

$c_{m} \quad$ resistência transversal da camada semiconfinante inferior do aqüífero $m$

d diâmetro médio dos grãos do meio

D domínio da solução

D tensor de proporcionalidade entre a descarga aparente e o campo gravitacional do escoamento

E campo gravitacional

e índice identificador de espelhos

el índice identificador de elementos

F função que satisfaz a teoria do potencial

$f, \mathrm{~g}, F, \mathrm{G} \quad$ funções

$g \quad$ aceleração da gravidade no local

$h \quad$ altura da lâmina de água do escoamento

$H \quad$ espessura do aqüífero

h vetor formado pelos elementos $\phi_{m}$

$H^{*} \quad$ espessura de paredes (estruturas horizontais) presentes no aqüífero

$H_{m} \quad$ espessura do aqüífero $m$

i indice da linha do elemento do tensor de condutividades do meio poroso

I integral

$i, j \quad$ índices utilizados na identificação de termos de uma série

$\hat{i}, \hat{j}, \hat{k}$ versores do sistema cartesiano de coordenadas

$j \quad$ indice da coluna do elemento do tensor de condutividades do meio poroso

$K \quad$ condutividade hidráulica do meio poroso saturado

$K^{-}$condutividade hidráulica em posições onde a parte imaginária da coordenada complexa adimensional encontra-se no limite negativo 
$k \quad$ permeabilidade intrínseca do material poroso; índice utilizado em séries

$K^{*} \quad$ condutividade hidráulica de paredes (estruturas horizontais) presentes no aqüífero

$K^{+} \quad$ condutividade hidráulica em posições onde a parte imaginária da coordenada complexa adimensional encontra-se no limite positivo

$K_{i j} \quad$ elementos do tensor de condutividades do meio poroso

$K_{m} \quad$ condutividade hidráulica do aqüífero $m$

$\underline{\mathbf{K}} \quad$ tensor de condutividades do meio poroso

L comprimento do tubo utilizado no Experimento de Darcy

$L_{m} \quad$ espessura da camada semiconfinante inferior do aqüífero $m$

$m \quad$ índice que identifica o aqüífero em um sistema de camadas aqǘferas

$n \quad$ número de termos de uma série

$N \quad$ taxa de recarga do aqüífero

$n_{e} \quad$ porosidade efetiva do meio

$N_{m} \quad$ taxa de recarga do aqüífero $m$

p pressão do fluido; polinômio de correção do potencial no infinito

pc ponto de controle

Q velocidade aparente do escoamento totalizada na direção vertical

q velocidade aparente do escoamento, ou seja, a descarga de água por unidade área perpendicular à sua direção

$Q^{*} \quad$ velocidades aparentes distribuídas ao longo de paredes (estruturas horizontais) presentes no aqüífero

$Q^{+} \quad$ velocidade aparente bidimensional em posições onde a parte imaginária da coordenada complexa adimensional encontra-se no limite positivo

$Q^{-} \quad$ velocidade aparente bidimensional em posições onde a parte imaginária da coordenada complexa adimensional encontra-se no limite positivo

$Q_{\xi}^{+} \quad$ componente horizontal da velocidade aparente bidimensional em posições onde a parte imaginária da coordenada complexa adimensional encontra-se no limite positivo

$Q_{\xi}^{-} \quad$ componente horizontal da velocidade aparente bidimensional em posições onde a parte imaginária da coordenada complexa adimensional encontra-se no limite positivo 
$Q_{\eta}^{+} \quad$ componente vertical da velocidade aparente bidimensional em relação a um segmento de reta em posições onde a parte imaginária da coordenada complexa adimensional encontra-se no limite positivo

$Q_{\eta}^{-} \quad$ componente vertical da velocidade aparente bidimensional em relação a um segmento de reta em posições onde a parte imaginária da coordenada complexa adimensional encontra-se no limite negativo

$q_{x} \quad$ velocidade aparente do escoamento na direção $x$

$q_{\mathrm{y}} \quad$ velocidade aparente do escoamento na direção $y$

$q_{z} \quad$ velocidade aparente do escoamento na direção $Z$

r posição relativa a um ponto

$R \quad$ reflexo obtido pelo Método de Imagens diante de uma determinada fronteira

$S \quad$ coeficiente de armazenamento bidimensional do aqüífero

$S$ conjunto de pontos obtidos de um domínio refletido mediante o Método de Imagens

s deslocamento do escoamento

$s \quad$ intensidade de um dipolo plano

$S_{0} \quad$ coeficiente de armazenamento específico para solos verticalmente consolidantes

$t$ instante de tempo onde o fenomeno ocorre

$T \quad$ transmissividade do aqüífero

$u, v, w$ variáveis auxiliares

$v \quad$ índice para identificação de vértices de uma poligonal

V volume de controle

W variável complexa que representa a velocidade aparente totalizada na vertical

$X \quad$ parte real da coordenada complexa adimensional

$x, y, z$ coordenadas do sistema cartesiano de localização no espaço

$Y \quad$ limite negativo da parte imaginária da coordenada complexa adimensional

$Y \quad$ parte imaginária da coordenada complexa adimensional

$Y^{+} \quad$ limite positivo da parte imaginária da coordenada complexa adimensional

Z coordenada complexa adimensional de um ponto em relação a um segmento de reta

Z $\quad$ coordenada do plano complexo

$z_{0} \quad$ coordenada complexa do centro geométrico de uma poligonal

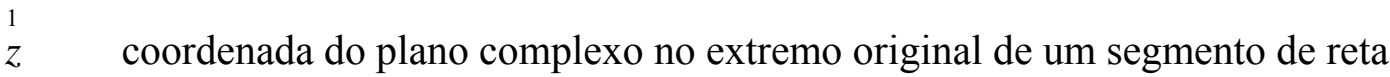


Z coordenada do plano complexo no extremo terminal de um segmento de reta

Zeta plano adimensional de mapeamento de elementos utilizados em Aqüíferos Longos

$\Delta \quad$ variável adimensional de integração sobre um segmento de reta

$\bar{\phi} \quad$ carga piezométrica média

$\stackrel{2}{\phi} \quad$ carga piezométrica na extremidade de maior abscissa do tubo utilizado no Experimento de Darcy; função qualquer que satisfaz equações lineares

$\stackrel{1}{\phi} \quad$ carga piezométrica na extremidade de menor abscissa do tubo utilizado no Experimento de Darcy; função qualquer que satisfaz equações lineares

$\gamma \quad$ produção de massa fluida por unidade de área; divergente

$v$
$\gamma$ produção de massa fluida por unidade de volume

$\Phi \quad$ potencial de descarga

$\Phi$ parcela do potencial de descarga devido a elementos de esforços calculados

$\underset{e}{\Phi} \quad$ parcela do potencial de descarga devido a elementos de esforços especificados

$\stackrel{k}{\Phi} \quad$ parcela do potencial de descarga devido a um efeito ocorrido em determinado instante

$\stackrel{b}{\Phi} \quad$ potencial de descarga do lado externo de um elemento Areasink

$\stackrel{a}{\Phi} \quad$ potencial de descarga do lado interno de um elemento Areasink

$\Phi_{\text {ss }} \quad$ potencial de descarga em regime estacionário em um ponto

$\Phi^{+} \quad$ potencial de descarga em posições onde a parte imaginária da coordenada complexa adimensional encontra-se no limite positivo

$\Phi^{-} \quad$ potencial complexo de descarga em posições onde a parte imaginária da coordenada complexa adimensional encontra-se no limite negativo

$\Gamma \quad$ condições da solução no contorno $C$

$\lambda_{j}^{e l} \quad$ coeficiente da distribuição de esforços de um dado elemento

$\Lambda \quad$ função de influência de um elemento

$\stackrel{e}{\Theta} \quad$ operação do Método de Imagens sobre um elementos 
$\Omega \quad$ potencial complexo de descarga

$\Omega^{+} \quad$ potencial complexo de descarga em posições onde a parte imaginária da coordenada complexa adimensional encontra-se no limite positivo

$\Omega^{-} \quad$ potencial complexo de descarga em posições onde a parte imaginária da coordenada complexa adimensional encontra-se no limite negativo

$\Xi \quad$ parte real de um ponto do plano Zeta

$\Psi \quad$ linhas de corrente

$\Psi^{+} \quad$ linhas de corrente em posições onde a parte imaginária da coordenada complexa adimensional encontra-se no limite positivo

$\Psi^{-} \quad$ linhas de corrente em posições onde a parte imaginária da coordenada complexa adimensional encontra-se no limite negativo

$\alpha \quad$ coeficiente de compressibilidade da água; ângulo de um segmento de reta; coeficiente de difusividade do escoamento

$\beta \quad$ coeficiente de compressibilidade do material poroso; rotacional da velocidade aparente; direção da intensidade do dipolo

$\chi$ constante real da Equação de Helmholtz; função paramétrica das linhas de corrente; potencial de Zhukovski; fun

$\delta \quad$ variável de integração sobre um segmento de reta

$\phi \quad$ carga, ou energia, piezométrica da água; função qualquer que satisfaz equações lineares

$\phi_{m} \quad$ carga, ou energia, piezométrica do aqüífero $m$

$\phi^{+} \quad$ carga piezométrica em posições onde a parte imaginária da coordenada complexa adimensional encontra-se no limite positivo

$\phi^{-} \quad$ carga piezométrica em posições onde a parte imaginária da coordenada complexa adimensional encontra-se no limite negativo

$\eta \quad$ ordenada de um ponto em relação ao centro de um segmento de reta ordenada de um ponto localizado em um plano utilizado para mapeamento

$\varphi \quad$ variável auxiliar

$\lambda$ função paramétrica das linhas de corrente; distribuição de esforços devidos a dipolos transversais a um dado segmento de reta

$\lambda \quad$ vetor de incógnitas dos esforços dos elementos 
$\mu \quad$ viscosidade dinâmica do fluido; distribuição de esforços devidos a dipolos alinhados a um segmento de reta

$\theta \quad$ ângulo; coordenada angular de um ponto

$\theta^{+} \quad$ coordenada angular de um ponto em posições onde a parte imaginária da coordenada complexa adimensional encontra-se no limite positivo

$\theta^{-}$coordenada angular de um ponto em posições onde a parte imaginária da coordenada complexa adimensional encontra-se no limite negativo

$\rho \quad$ massa específica do fluido

$\sigma \quad$ distribuição da taxa de descarga ao longo de um segmento de reta

$\tau \quad$ instante de tempo inicial de integração

$\tau \quad$ variável de integração temporal

$\omega \quad$ coeficientes presentes nos termos de uma equação diferencial

$\xi \quad$ abscissa de um ponto em relação ao centro de um segmento de reta; abscissa de um ponto localizado em um plano utilizado para mapeamento

$\zeta \quad$ plano de coordenadas complexas em relação ao centro de um segmento de reta; ponto localizado em um plano utilizado para mapeamento

I parte imaginária de um número (variável) complexo

$\Re \quad$ parte real de um número (variável) complexa 


\section{Sumário}

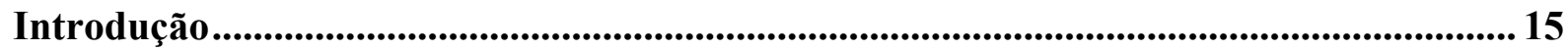

\section{Capítulo 1}

FUNDAMENTOS DO ESCOAMENTO DE ÁGUA EM AQÜÍFEROS ................... 17

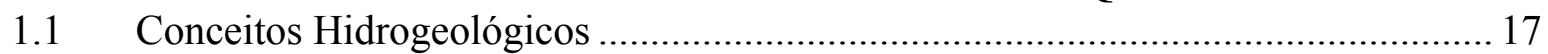

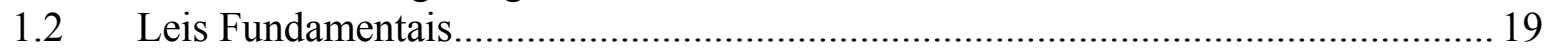

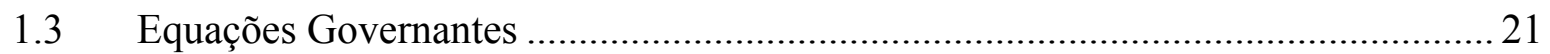

\section{Capítulo 2}

FERRAMENTAS ANALÍTICAS EM ÁGUAS SUBTERRÂNEAS ........................... 27

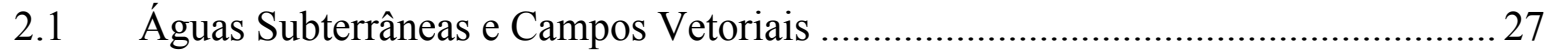

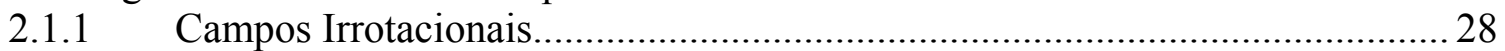

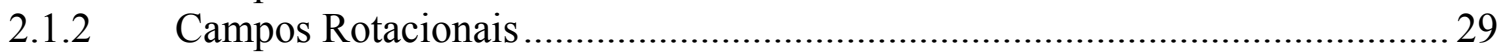

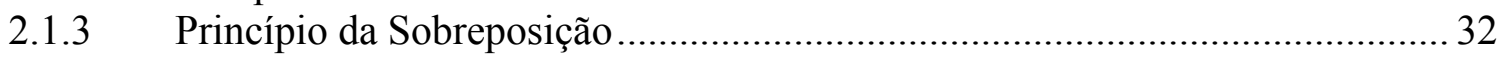

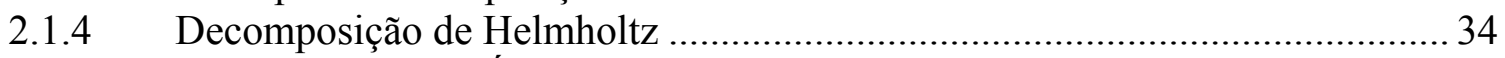

2.2 Funções Analíticas em Águas Subterrâneas........................................................... 35

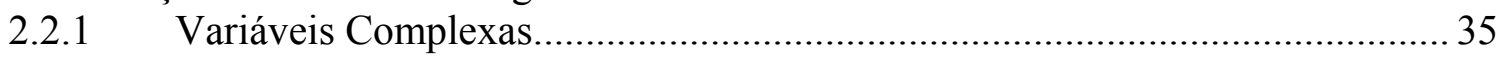

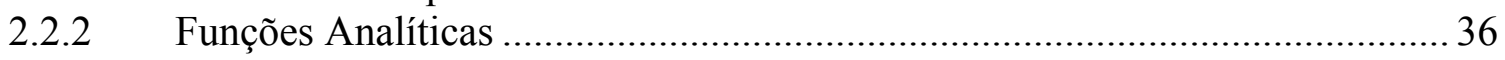

2.2.3 Funções Harmônicas ...................................................................................... 37

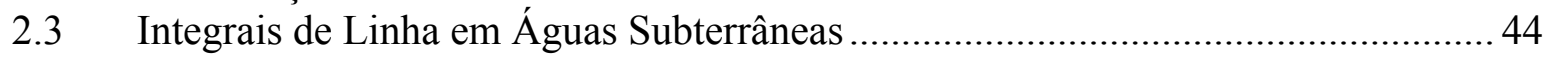

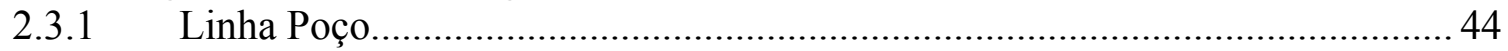

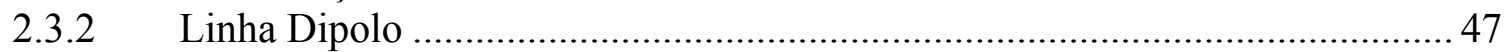

2.3.3 Integração do Potencial de Newton sobre uma Linha....................................... 49

2.3.4 Integral do Dipolo Elétrico sobre uma Linha...................................................50

2.3.5 Integração do Potencial de Newton sobre um Anel .......................................... 51

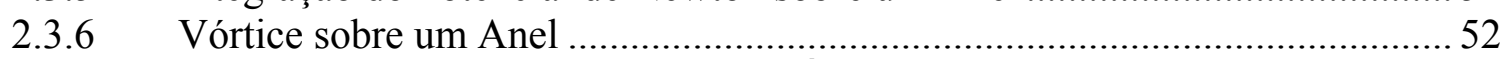

2.4 Representação de Fronteiras Externas em Águas Subterrâneas................................. 53

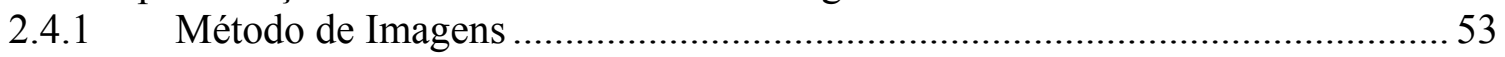

2.4.2 Transformações de Schwarz-Christoffel ..................................................... 54

Capítulo 3

MÉTODO DE ELEMENTOS ANALÍTICOS .......................................................... 58

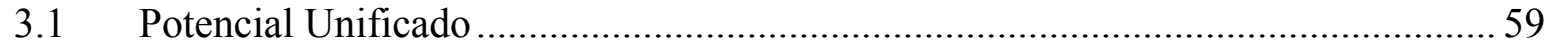

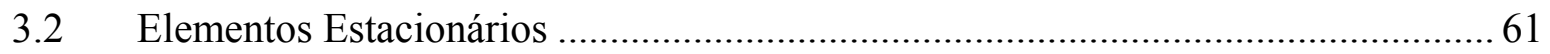

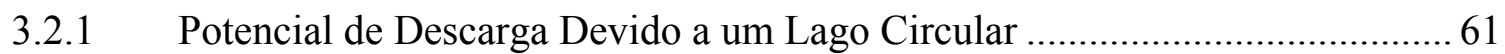

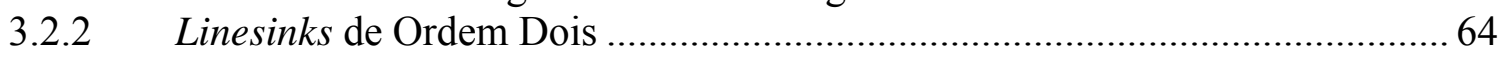

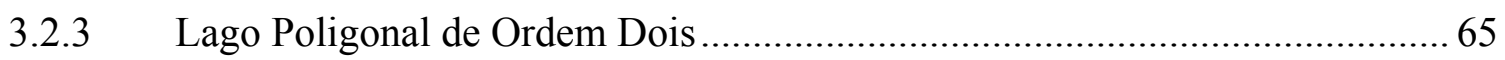

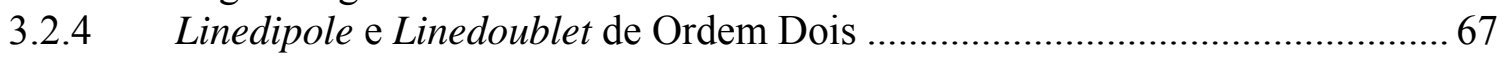

3.2.5 Areasink de Esforço Uniformemente Distribuído ............................................ 72

3.3 Elementos Transientes..................................................................................... 78

3.3.1 Potencial de Descarga Devido a um Lago Circular ........................................ 79

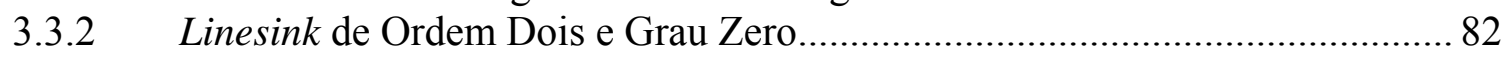

3.3.3 Lago Poligonal de Ordem Dois e Grau Zero.................................................. 89

3.3.4 Linedoublet de Ordem Dois e Grau Zero ......................................................... 90

3.3.5 Areasink Ordem Zero e Grau Zero.................................................................... 95

3.4 Condições de Contorno .................................................................................... 98

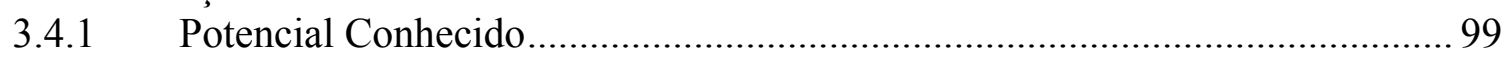

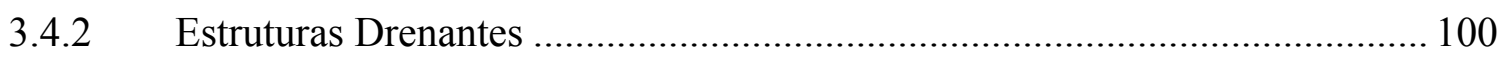

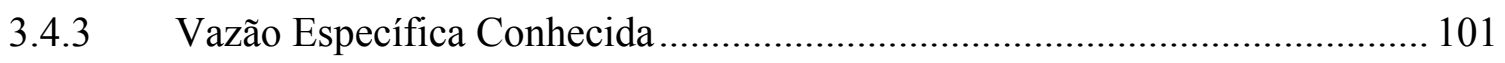

3.4.4 Condições de Contorno Combinadas ............................................................... 103 


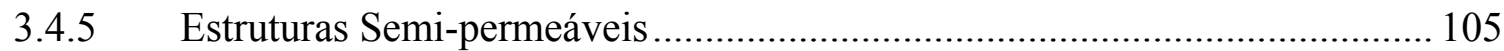

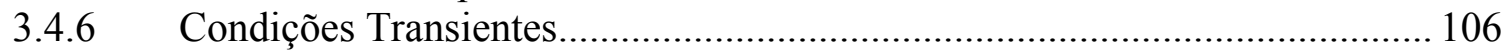

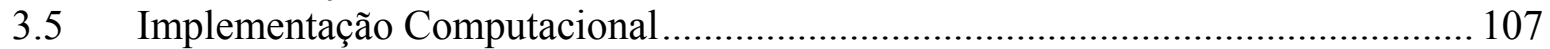

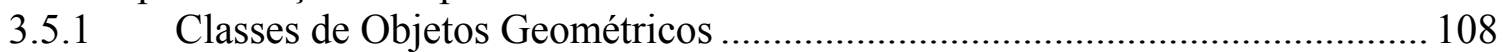

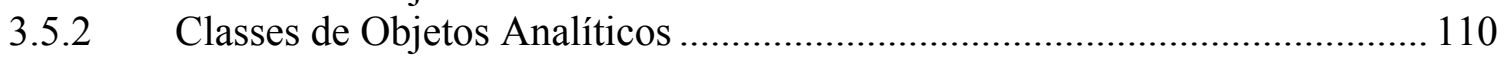

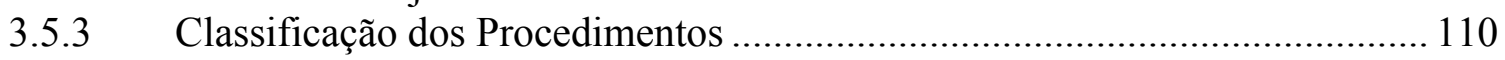

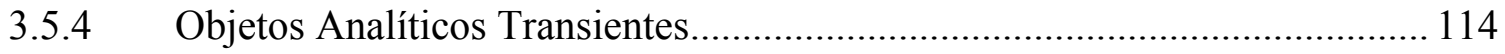

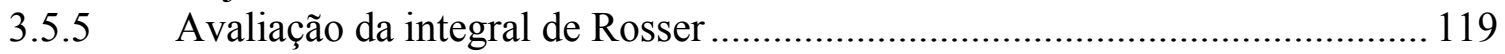

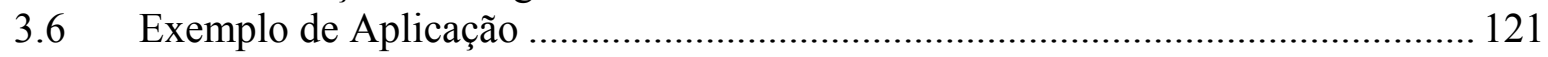

\section{Capítulo 4}

ALTERNATIVAS AO USO DE ELEMENTOS EM MODELOS DE AQÜÍFEROS

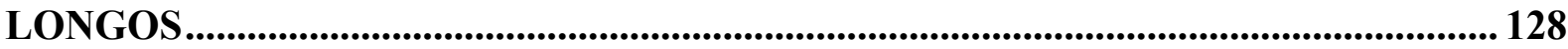

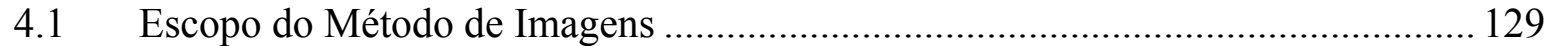

4.2 Transformações de Schwarz-Christoffel para Duas Fronteiras Retilíneas............. 132

4.3 Uso de Elementos Analíticos em Domínios Alongados ......................................... 136

4.3.1 Recarga Direta Compreendida por Duas Fronteiras Paralelas ........................ 137

4.3.2 Aplicação do Método de Imagens sobre Elementos Analíticos ...................... 138

4.3.3 Aplicação de Transformações de Schwarz-Christoffel sobre Elementos

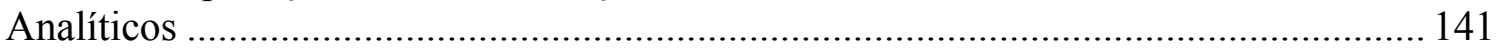

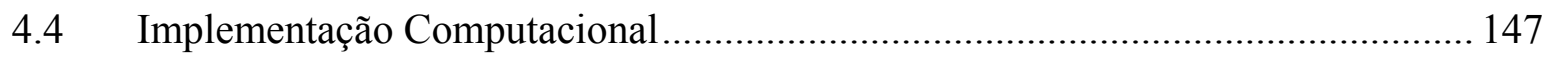

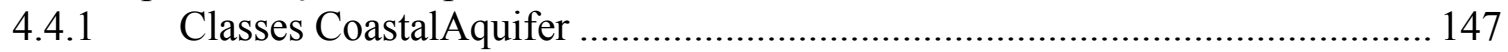

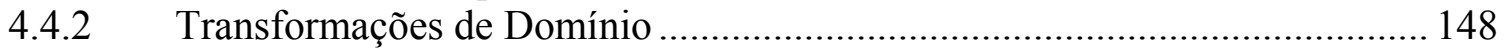

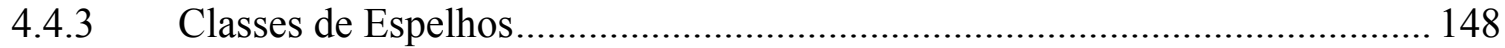

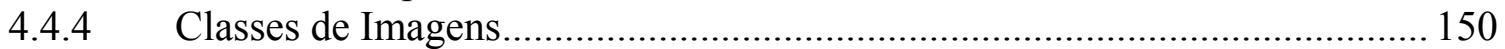

4.4.5 Procedimento de Construção de Objetos Imagens .......................................... 152

4.4.6 Procedimentos de Solução nos Objetos Imagens ........................................... 152

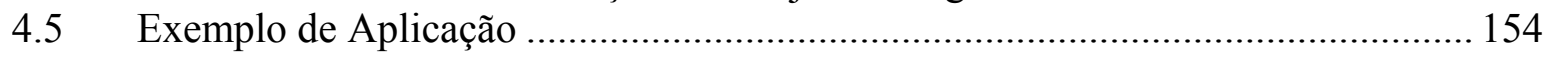

\section{Capítulo 5}

IMPACTO DA CAPTAÇÃO DA ADUTORA AGRESTE/TRAIRI/POTENGI

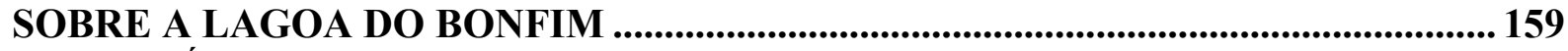

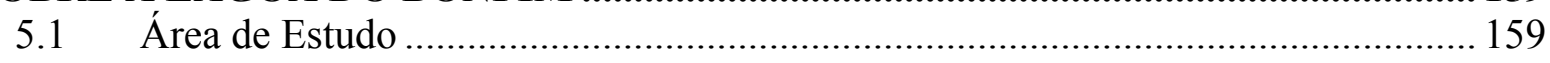

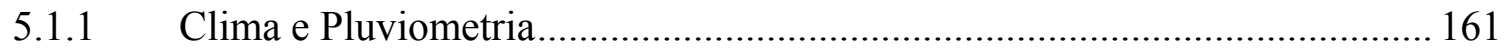

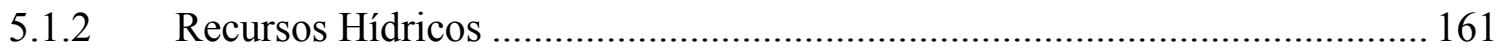

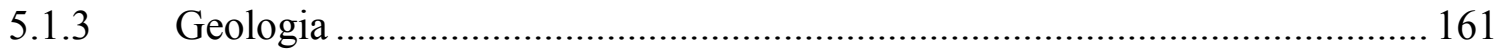

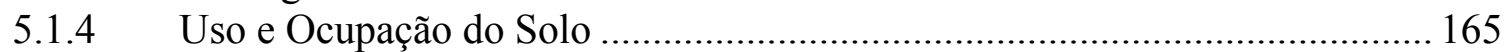

5.1.5 Estudos Anteriores de Potenciometria na Área de Estudo ................................. 167

5.1.6 Estudos Anteriores de Impactos sobre a Lagoa do Bonfim ............................ 170

5.2 Modelagem do Escoamento Subterrâneo e Calibração........................................... 171

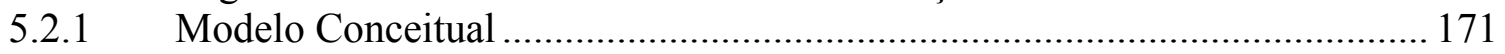

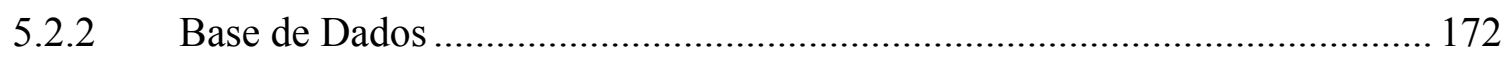

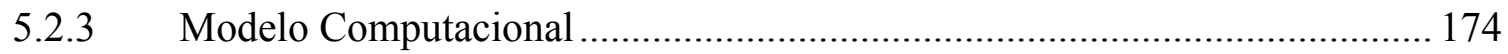

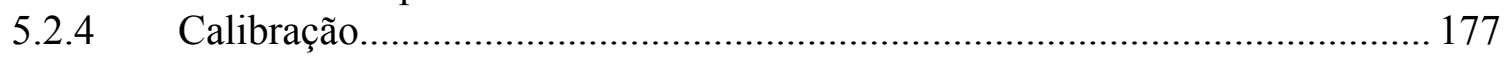

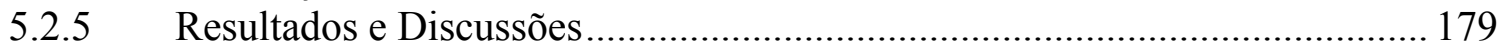

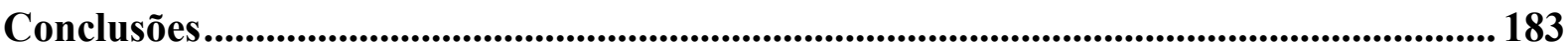

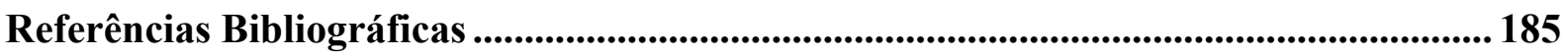

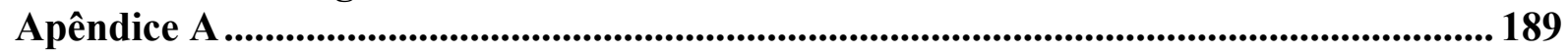

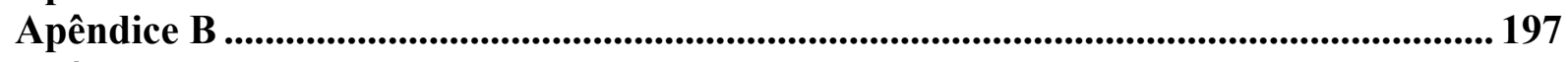

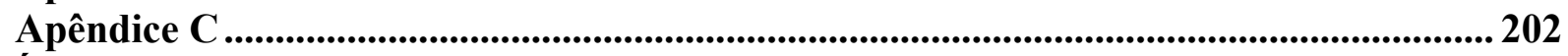

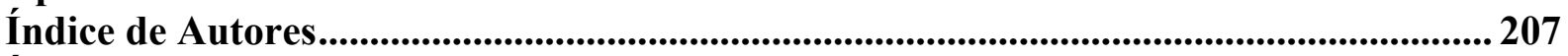

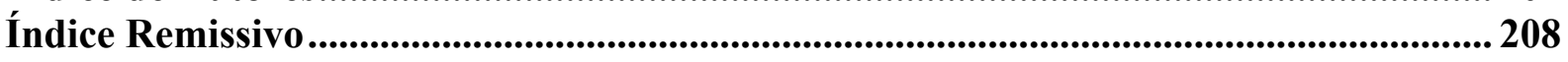




\section{Introdução}

A modelagem de escoamento tem sido amplamente utilizada para o planejamento e a gestão de recursos hídricos. A necessidade da utilização de modelos para esses fins tem crescido à medida que cresce a demanda desses recursos. As formações geológicas onde é possível a exploração de águas subterrâneas, geralmente, estendem-se muito além das regiões para as quais são construídos os modelos. Sob essas circunstâncias, lança-se mão do uso de fronteiras artificiais para limitar o domínio do modelo. Alternativamente à "prática" de utilização de fronteiras artificiais, o Método de Elementos Analíticos (na literatura internacional, Analytic Element Method ou AEM) é formulado em termos de funções analíticas, e portanto, é adequado para a modelagem de regiões abertas (Strack, 1989).

Essas formações geológicas comumente apresentam-se, ainda, em formas alongadas, ou seja, estreitas em relação à sua direção de maior comprimento. Entretanto, a representação de fronteiras de domínios semi-infinitos por meio do AEM comumente acarreta dúvidas quanto aos critérios a serem adotados para se evitar eventuais efeitos de borda. Aqüíferos com essa conformação são chamados neste trabalho de Aqüíferos Longos e podem ser caracterizados por meio de uma faixa de domínio infinita, sobre a qual componentes bi ou tridimensionais do escoamento podem ser modeladas. No Brasil, o Aqüífero Barreiras compreende uma largura muito pequena em relação à sua extensão e, portanto, constitui-se em um exemplo de Aqüífero Longo.

No Estado do Rio Grande do Norte, o Aqüífero Barreiras fornece água para diversos sistemas de abastecimento público de água. O Sistema Adutor Agreste/Trairi/Potengi possui o maior sistema de captação instalado na porção do Aqüífero gerenciada pelo Estado. A captação do Sistema encontra-se instalada na regão do Sistema Lacustre Bonfim, onde encontram-se, ainda, diversos córregos e lagoas utilizados para atividades recreativas. Os impactos ambientais do sistema têm sido amplamente discutidos na sociedade, bem como na comunidade técnico-científica, desde sua fase de concepção, em 1996, até hoje. No entanto, embora relatórios de consultoria apontem poços de captação alternativos (e.g. Manuel Filho e Castro, 2002), a modelagem da hidrodinâmica do sistema realizada em estudos anteriores não tem reproduzido o comportamento observado em campo (Pereira et al., 2003).

Este trabalho tem o objetivo de, em primeiro lugar, estender o Método de Elementos Analíticos para a modelagem de escoamentos em Aqüíferos Longos, uma configuração de domínio ainda não abordada na literatura do método. Em segundo lugar, este trabalho visa 
descrever o escoamento subterrâneo da região do Sistema Lacustre Bonfim, fazendo simulações baseadas em Elementos Analíticos e, assim, introduzir novos subsídios à discussão sobre a hidrodinâmica da área de estudo. A modelagem baseada em Elementos Analíticos permite o enriquecimento da análise do escoamento à medida que são utilizadas funções explícitas para cada efeito introduzido ao modelo. Portanto, esse trabalho contribui tanto para a extensão do Método de Elementos Analíticos, ampliando-se o seu alcance de aplicação, como para o conhecimento da hidrodinâmica do Sistema Lacustre Bonfim.

No primeiro capítulo, apresenta-se os conceitos e equações fundamentais no estudo de escoamentos em meios porosos no âmbito da Engenharia de Recursos Hídricos. No segundo capítulo, apresenta-se uma visão geral sobre ferramentas analíticas comumente utilizadas no estudo do escoamento de águas subterrâneas. No terceiro capítulo, o Método de Elementos Analíticos é apresentado. Como contribuição ao estado-da-arte do método são revistas algumas equações utilizadas na literatura e introduzidos aperfeiçoamentos de modo a corrigir expressões que descrevem de forma insatisfatória o comportamento de alguns elementos. No quarto capítulo, é desenvolvida uma nova formulação para a simulação de escoamento em Aquiferos Longos baseada no Método de Elementos Analíticos. Observa-se que essa técnica dispensa o uso de elementos em fronteiras (externas) permitindo, também, que a definição de elementos nos contornos internos do Aquiferos Longos seja feita de maneira apropriada. Por fim, no quinto capítulo, a metodologia desenvolvida é testada em um problema real construindo-se um modelo de Elementos Analíticos para o estudo do escoamento subterrâneo no Sistema Lacustre Bonfim. Embora a implantação da captação tenha motivado diversos estudos, o presente trabalho contribui para a questão, fornecendo uma calibração dos parâmetros hidráulicos e simulando cenários de seca na região. 


\section{Capítulo 1}

\section{FUNDAMENTOS DO ESCOAMENTO DE ÁGUA EM AQÜÍFEROS}

Neste capítulo são apresentados conceitos e equações fundamentais para o estudo de escoamentos em meios porosos.

\subsection{Conceitos Hidrogeológicos}

A água existe em uma quantidade finita e é encontrada nos seus três estados físicos na atmosfera, sobre a superfície terrestre e no interior de nosso subsolo. $O$ estudo de sua ocorrência, movimentação e reações com o meio ambiente, define, em poucas palavras, o objeto da Hidrologia (Villela e Mattos, 1975). De maneira geral, as rochas encontradas no subsolo são constituídas de materiais porosos, capazes de absorver água. Penetrando-se a superfície terrestre após uma certa profundidade, os poros do material perfurado serão encontrados completamente preenchidos com água. A água encontrada nos poros saturados do subsolo é denominada Água Subterrânea (Fetter, 1994).

Hidrogeologicamente, rochas e solos são classificados de acordo com sua capacidade de transmitir água. Formações impermeáveis recebem a denominação de Aqüicludes. Formações geológicas com baixa permeabilidade são denominadas Aqüitardes. Finalmente, os Aqüíferos constituem-se formações capazes de armazenar e transmitir água suficiente para fornecer quantidades razoáveis para poços (Fetter, 1994).

A ocorrência de águas subterrâneas pode ser observada com baixa concentração de sais (doce) ou com uma concentração elevada de sais dissolvidos das rochas (salobra). Na Tabela 1.1.1 apresenta-se a distribuição da água na Terra destacando a ocorrência de águas subterrâneas. Os valores entre parênteses referem-se a subtotais da linha superior. Reservas permanentes de gelo, vapor de água na atmosfera e água nos seres vivos não estão disponíveis para o abastecimento humano. Pode-se inferir da Tabela 1.1.1 que as águas subterrâneas doces constituem $96,3 \%$ de toda água doce disponível no planeta. Somente 3,7\% de toda água disponível (rios, lagos e pântanos) encontram-se sobre os continentes. 
Tabela 1.1.1 - Distribuição da água no ciclo hidrológico.

\begin{tabular}{l|l|l|l}
\hline Fonte Hídrica & $\begin{array}{c}\text { Volume } \\
\left(1.000 \mathrm{~km}^{3}\right)\end{array}$ & $\begin{array}{l}\text { Percentual } \\
\text { do Volume } \\
\text { Total }\end{array}$ & $\begin{array}{l}\text { Percentual } \\
\text { do Volume } \\
\text { Doce }\end{array}$ \\
\hline $\begin{array}{l}\text { Oceanos, Mares e } \\
\text { Baías. }\end{array}$ & 1.338 .000 & 96,5 & - \\
\hline $\begin{array}{l}\text { Capas e Montanhas } \\
\text { de Gelo e Neve } \\
\text { Permanentes }\end{array}$ & 24.064 & 1,74 & 68,7 \\
\hline Água Subterrânea & 23.400 & 1,7 & - \\
\hline Doce & $(10.530)$ & $(0,76)$ & 30,1 \\
\hline Salobra & $(12.870)$ & $(0,94)$ & - \\
\hline Umidade do Solo & 16,5 & 0,001 & 0,05 \\
\hline "permafrost" & 300 & 0,022 & 0,86 \\
\hline Lagos & 176,4 & 0,013 & - \\
\hline \multicolumn{1}{c}{ Doce } & $(91,0)$ & $(0,007)$ & 0,26 \\
\hline Salobra & $(85,4)$ & $(0,006)$ & - \\
\hline Atmosfera & 12,9 & 0,001 & 0,04 \\
\hline Pântanos & 11,47 & 0,0008 & 0,03 \\
\hline Rios & 2,12 & 0,0002 & 0,006 \\
\hline Seres Vivos & 1,12 & 0,0001 & 0,003 \\
\hline Total & 1.385 .984 & 100,0 & 100,0 \\
\hline $\begin{array}{l}\text { Fonte: Gleick, P. H., } 1996: \text { Water resources. In. Schneider, S. H } \\
\text { ed. Encyclopedia of Climate and Weather, Oxford University Press, }\end{array}$ \\
New York, vol. 2, pp.817-823.
\end{tabular}

Em toda Terra, a água é submetida a uma renovação dinâmica e perene com diferentes intensidades e escalas de tempo de acordo com o ambiente onde se encontra. De uma forma geral, ambientes onde a água é encontrada com maior abundância detêm a água durante maiores intervalos de tempo, como é o caso das águas subterrâneas. $\mathrm{O}$ inverso acontece em meios como a atmosfera, onde a água permanece menos tempo. Portanto, em nenhum meio que contém água no ambiente o armazenamento é estático. Ou seja, as águas de qualquer meio estão em movimento e trocam massas continuamente entre as diversas componentes encontradas no ciclo hidrológico.

Em ambientes hidrogeológicos (aqüíferos), a água escoa de forma muito lenta $(\mathrm{cm} / \mathrm{dia}$ ou m/dia) se comparada ao escoamento superficial ou ao atmosférico, função da resistência imposta pelos poros das rochas. Hidrologicamente, a resistência das rochas ao escoamento é necessária para que as cargas piezométricas dos aqüíferos sejam mantidas em níveis elevados. Dessa forma, a água subterrânea interage com rios, lagos e nascentes tornando-os perenes. A gestão de recursos hídricos subterrâneos, portanto, deve ser responsável pela manutenção das cargas piezométricas próximas às condições naturais, a fim de promover o manejo sustentável dos recursos hídricos. 


\subsection{Leis Fundamentais}

Henry Darcy, em 1856, quantificou a perda de carga piezométrica necessária para a produção de determinados valores de descarga de água em um cilindro de areia de comprimento fixo. A descarga mostrou-se diretamente proporcional à perda de energia e inversamente proporcional ao comprimento do cilindro. As variáveis estudadas estariam relacionadas por intermédio de uma constante de proporcionalidade $K$, representativa das propriedades do fluido e do meio.

$$
\mathbf{q}=-K^{\frac{2}{\phi}-\phi}
$$

A expressão traz $\mathbf{q}\left[\mathrm{LT}^{-1}\right]$ como a descarga por unidade de área transversal, $\stackrel{1}{\phi}$ e $\stackrel{2}{\phi}$ [L] como as cargas piezométricas nas extremidades do tubo, $L[\mathrm{~L}]$, o comprimento do percurso e $K\left[\mathrm{LT}^{-1}\right]$, a condutividade hidráulica. Reescrevendo-se a Eq. 1.2.1 na forma diferencial, a expressão resultante é fisicamente interpretada como a aplicação da lei de conservação da energia piezométrica $(\phi)$ do fluido em meios porosos sobre um eixo $x$.

$$
\mathbf{q}=-K \frac{d \phi}{d x}
$$

Esse ponto de vista permite interpretar a condutividade hidráulica $K$ como o produto de $k$, a permeabilidade intrínseca $\left[\mathrm{L}^{2}\right]$ do meio pelo fator hidrodinâmico $\rho g / \mu$ do fluido,

$$
K=k . \rho g / \mu
$$

onde $\rho g$ é o peso específico do fluido $\left[\mathrm{MT}^{-2} \mathrm{~L}^{-2}\right]$ e $\mu$ a viscosidade dinâmica $\left[\mathrm{MT}^{-1} \mathrm{~L}^{-1}\right]$. Aplicando-se as equações de conservação de quantidade de movimento do fluido (Equações de Navier-Stokes) à escala dos poros, Hubbert (1956) obteve a seguinte expressão para a permeabilidade intrínseca do meio:

$$
k=\frac{d^{2} n_{e}^{3}}{54(1-n)^{2}}
$$


onde $n_{e}$ é a porosidade efetiva do meio e $d$ o diâmetro médio dos grãos.

A equação do movimento do fluido no meio poroso orientada em um sistema de eixos $(x, y, z)$ cartesiano é chamada lei de Darcy generalizada, e é dada pela seguinte expressão:

$$
\mathbf{q}=-\left[\begin{array}{lll}
K_{x x} & K_{x y} & K_{x z} \\
K_{y x} & K_{y y} & K_{y z} \\
K_{z x} & K_{z y} & K_{z z}
\end{array}\right] \nabla \phi, \text { ou } \mathbf{q}=-\underline{\underline{\mathbf{K}} \nabla \phi}
$$

onde a matriz $\left[K_{i j}\right](i=x, y, z$ e $j=x, y, z)$ é o tensor de condutividades do meio. Mesmo em casos onde os eixos de coordenadas não coincidem com as direções principais de condutividade, observa-se (Bear, 1972 pág. 136) que o tensor de condutividades do meio é sempre simétrico em relação à diagonal principal $\left(K_{i j}=K_{j i}\right)$. Caso haja coincidência entre os eixos de coordenadas e os eixos principais de condutividade, o tensor é dado por uma matriz diagonal. Em ambos os casos, cada ponto do meio oferece diferentes componentes de condutividade para cada componente do escoamento. Formações geológicas assim constituídas são denominadas aqüíferos anisotrópicos. Essa é uma situação comumente encontrada em formações geológicas estratificadas e em formações fraturadas.

Reescrevendo a equação Eq. 1.2.5 para cada eixo de coordenadas, tem-se:

$$
\left\{\begin{array}{l}
q_{x}=-K_{x x} \frac{\partial \phi}{\partial x}-K_{x y} \frac{\partial \phi}{\partial y}-K_{x z} \frac{\partial \phi}{\partial z} \\
q_{y}=-K_{y x} \frac{\partial \phi}{\partial x}-K_{y y} \frac{\partial \phi}{\partial y}-K_{y z} \frac{\partial \phi}{\partial z} \\
q_{z}=-K_{z x} \frac{\partial \phi}{\partial x}-K_{z y} \frac{\partial \phi}{\partial y}-K_{z z} \frac{\partial \phi}{\partial z}
\end{array}\right.
$$

Quando são observados valores iguais de condutividade em todas as direções de cada ponto do meio, o tensor da equação Eq. 1.2.5 torna a ser expresso novamente por um escalar. As formações assim caracterizadas são denominadas hidrogeologicamente de aqüíferos isotrópicos. Meios isotrópicos são comumente encontrados em formações geológicas sedimentares recentes. 
$\mathbf{q}=-K \nabla \phi$

A condutividade de uma formação isotrópica é, portanto, expressa por um único valor em todas as direções de um único ponto. A condutividade hidráulica pode, por outro lado, variar entre dois pontos em duas situações: 1) em uma formação que apresenta uma variação suave de sua condutividade; 2) considerando-se dois pontos localizados sobre formações diferentes. No primeiro caso, a caracterização da condutividade é feita, por exemplo, por meio de métodos geoestatísticos. No segundo, são identificadas regiões diferentes de mesma condutividade. Em ambos os casos, as formações constituem-se meios denominados aqüíferos heterogêneos e sua condutividade $K$ é considerada uma função do espaço.

Para o fechamento do equacionamento das incógnitas $\mathbf{q}$ e $\phi$ é necessário ainda, observar-se a continuidade da massa de fluido, especificando-se então o campo de escoamento e de alturas piezométricas $\phi$ (o campo piezométrico). A expressão da conservação de uma massa fluida de densidade constante é dada pela Equação da Continuidade escrita da seguinte forma:

$$
\vec{\nabla} \cdot \mathbf{q}+S_{0} \frac{\partial \phi}{\partial t}=0
$$

onde $S_{0}\left[\mathrm{~L}^{-1}\right]$ é o coeficiente de armazenamento específico para solos verticalmente consolidantes. $\mathrm{O}$ coeficiente $\mathrm{S}_{0}$ é definido como o volume de água liberado/recebido dos poros do aqüífero por uniade de volume do material poroso para cada unidade de variação de carga piezométrica no aqüífero, dado por $S_{0}=\rho g[\alpha(1-n)+n \beta]$ com $\alpha$ e $\beta$ sendo os coeficientes de compressibilidade do solo e da água, respectivamente (Bear, 1972).

\subsection{Equações Governantes}

O acoplamento das equações Eq. 1.2.7 e Eq. 1.2.8 fornece a equação governante que descreve de forma geral, para o caso onde os eixos de coordenadas encontram-se alinhados aos eixos principais de condutividade, o escoamento de águas subterrâneas.

$$
\frac{\partial}{\partial x}\left(K_{x x} \frac{\partial \phi}{\partial x}\right)+\frac{\partial}{\partial y}\left(K_{y y} \frac{\partial \phi}{\partial y}\right)+\frac{\partial}{\partial z}\left(K_{z z} \frac{\partial \phi}{\partial z}\right)=S_{0} \frac{\partial \phi}{\partial t}
$$


A aplicação da Eq. 1.3.1 exige mais conhecimento sobre as propriedades físicas do aqüífero e suas condições de contorno do que geralmente se dispõe. No entanto, determinados casos particulares permitem que sejam introduzidas simplificações que conduzem o equacionamento a algumas equações diferenciais amplamente estudadas.

Em aqüíferos confinados, a seção transversal ao escoamento é considerada constante no tempo e a distribuição de pressão hidrostática, ou seja, as linhas equipotenciais na seção são independentes da posição $z$. Dessa forma, o termo referente à variação de $\phi$ na direção do eixo $z$ torna-se nulo na equação geral (Eq. 1.3.1). Reescrevendo-se, então, a Eq. 1.3.1 nas duas dimensões restantes e adicionado um termo de fonte $N$ que expressa a passagem de água através das superfícies de topo e de base do aqüífero, tem-se:

$$
\frac{\partial}{\partial x}\left(K b \frac{\partial \phi}{\partial x}\right)+\frac{\partial}{\partial y}\left(K b \frac{\partial \phi}{\partial y}\right)+N=S \frac{\partial \phi}{\partial t}
$$

onde $K$ é a condutividade hidráulica; $H$ é a espessura do aqüífero; $S$, o coeficiente de armazenamento definido como $S=S_{0} b$ e $N\left[\mathrm{LT}^{-1}\right]$, a função de distribuição espacial e temporal da recarga.

Por sua vez, fazendo o termo à direita do sinal de igualdade na Eq. 1.3.2 igual a zero, o escoamento permantente confinado torna-se expresso por:

$$
\frac{\partial}{\partial x}\left(K H \frac{\partial \phi}{\partial x}\right)+\frac{\partial}{\partial y}\left(K H \frac{\partial \phi}{\partial y}\right)+N=0
$$

Em aqüíferos livres, ao observar-se que de modo geral as propriedades do escoamento subterrâneo variam suavemente, Dupuit (1863 apud Bear, 1972) ${ }^{1}$ afirmou que (1) a perda de carga na direção do escoamento é igual à queda de altura de água; (2) a direção dos vetores de descarga é quase horizontal; e (3) as linhas equipotenciais na seção independem da posição $z$ $(\phi=p / \rho g+z$, onde $p$ é aproximada pela pressão hidrostática, $p=(\phi-z) \rho g)$. Assim, em aqüíferos livres onde são satisfeitas as suposições de Dupuit, a equação Eq. 1.3.1 torna-se também bidimensional, porém não-linear, uma vez que $b$ torna-se igual a $\phi$. A princípio, as

${ }^{1}$ Dupuit, J. (1863) Études Théoriques et Pratiques sur le Mouvment des Eaux dans les Canaux Découverts et à Travers les Terrains Permeables, $2^{\mathrm{a}}$ ed. , Dunod, Paris 
suposições de Dupuit foram introduzidas para problemas de escoamentos estacionários $\left(\frac{\partial \phi}{\partial t}=0\right)$. Assim, o escoamento permanente livre torna-se governado pela seguinte equação:

$$
\frac{\partial}{\partial x}\left(\frac{K}{2} \frac{\partial \phi^{2}}{\partial x}\right)+\frac{\partial}{\partial y}\left(\frac{K}{2} \frac{\partial \phi^{2}}{\partial y}\right)+N=0
$$

Boussinesq (1903, 1904 apud Bear, 1972) 2, 3 aplica as suposições de Dupuit a problemas de escoamento em regime transiente $\left(\frac{\partial \phi}{\partial t} \neq 0\right)$ admitindo o meio homogêneo. A partir das simplificações de Dupuit obtém-se a Equação da Continuidade da seguinte forma (Bear, 1972 pág. 374):

$$
-\frac{\partial \mathbf{Q}_{x}}{\partial x}-\frac{\partial \mathbf{Q}_{y}}{\partial y}+N=n_{e} \frac{\partial \phi}{\partial t}
$$

Utilizando-se a lei de Darcy (Eq. 1.2.7) e definindo-se $\mathbf{Q}$ como a soma das componentes de descarga $\mathbf{q}_{x}$ e $\mathbf{q}_{y}$ totalizada ao longo da espessura $H$, com base na segunda hipótese de Dupuit, e fazendo $\phi=H$ com base na hipótese três, tem-se em meios homogêneos:

$$
\frac{K}{2}\left[\frac{\partial^{2} \phi^{2}}{\partial x^{2}}+\frac{\partial^{2} \phi^{2}}{\partial y^{2}}\right]+N=n_{e} \frac{\partial \phi}{\partial t}
$$

onde o coeficiente de armazenamento é dado pela porosidade efetiva do aqüífero $\left(n_{e}\right)$. A equação (Eq. 1.3.6) é conhecida como Equação de Boussinesq e também pelo nome do pesquisador Forchheimer (1930 apud Bear, 1972) ${ }^{4}$ devido aos trabalhos de ambos na solução

${ }^{2}$ Boussinesq, J. (1903) Recherches theóriques sur l'écoulement des nappes d'eau infiltrées dans le sol et sur debit de sources, J. Math. Pures Appl., CRH Acad. Sci., v10, pp5-78;

${ }^{3}$ Boussinesq, J. (1904) Recherches theóriques sur l'écoulement des nappes d'eau infiltrées dans le sol et sur debit de sources, J. Math. Pures Appl., CRH Acad. Sci., v10, pp363-394

${ }^{4}$ Forchheimer, P. (1930) Hydraulik, $3^{\mathrm{a}}$ ed. Teubner, Leipzig, Berlin 
da equação. De modo semelhante, admitindo-se a homogeneidade da condutividade e da espessura de aqüíferos confinados, a equação Eq. 1.3.2 é reduzida à forma seguinte:

$$
K H\left[\frac{\partial^{2} \phi}{\partial x^{2}}+\frac{\partial^{2} \phi}{\partial y^{2}}\right]+N=S \frac{\partial \phi}{\partial t}
$$

ou ainda,

$$
\frac{K H}{S}\left[\frac{\partial^{2} \phi}{\partial x^{2}}+\frac{\partial^{2} \phi}{\partial y^{2}}\right]+N / S=\frac{\partial \phi}{\partial t} .
$$

onde o termo $\frac{K H}{S}$ é conhecido como difusividade do aqüífero e a equação é referida como Equação do Calor.

Reduzindo-se, ainda as equações Eq. 1.3.6 e Eq. 1.3.8 ao regime estacionário, produz-se a Equação de Poisson, expressa para o escoamento livre e confinado, respectivamente, por :

$$
\begin{aligned}
& \frac{\partial^{2} \phi^{2}}{\partial x^{2}}+\frac{\partial^{2} \phi^{2}}{\partial y^{2}}=-2 N / K \\
& \frac{\partial^{2} \phi}{\partial x^{2}}+\frac{\partial^{2} \phi}{\partial y^{2}}=-N / K H
\end{aligned}
$$

$\mathrm{Na}$ ausência do termo de fonte, as equações Eq. 1.3.9 e Eq. 1.3.10 são reduzidas à forma da Equação de Laplace, respectivamente equações Eq. 1.3.11 e Eq. 1.3.12. Na equação geral Eq. 1.3.1, a suposição de meio homogêneo, isotrópico e inelástico produz igualmente a forma da Equação de Laplace .

$$
\begin{aligned}
& \nabla^{2} \phi^{2}=0 \\
& \nabla^{2} \phi=0
\end{aligned}
$$


Em aqüíferos estratificados, por outro lado, comumente são encontradas formações pouco permeáveis interpostas a formações de maior condutividade. Nesse caso, as formações de maior condutividade são chamadas de aqüíferos semiconfinados e a camada pouco condutiva, semiconfinante, constituindo-se uma camada que transmite água apenas em sua direção transversal. A ocorrência alternada dessas camadas configura os sistemas multicamada, onde a perda ou recarga $\left(N_{i}\right)$ do aqüífero semiconfinado para o(s) aqüífero(s) adjacente(s) é obtida em função da piezometria dos aqüíferos relacionados. A relação de recarga ou perda entre os aqüíferos, por sua vez, é obtida aplicando-se a expressão de Darcy (Eq. 1.2.1) para as camadas pouco condutoras adjacentes ao aqüífero semiconfinado. Portanto, pode-se escrever o termo de fonte da expressão Eq. 1.3.10, considerando-se a presença de duas camadas semiconfinantes, da seguinte maneira:

$$
N_{m}=\left(\frac{\phi_{m}-\phi_{m-1}}{C_{m-1}}\right)+\left(\frac{\phi_{m}-\phi_{m+1}}{c_{m}}\right)
$$

onde $m$ é o índice que numera cada camada de aqüífero, $c_{m-1}=\frac{H_{m-1}}{K_{m-1}}$ [T] é a resistência transversal da camada semiconfinante inferior do aqüífero definida em função de sua espessura $H_{m-1}$ e sua condutividade hidráulica $K_{m-1}$. Assim, a equação governante torna-se expressa da seguinte maneira:

$$
\frac{\partial^{2} \phi_{m}}{\partial x^{2}}+\frac{\partial^{2} \phi_{m}}{\partial y^{2}}=-N_{m} / K_{m} H_{m} \text { ou } \nabla^{2} \phi=\mathbf{A} \phi
$$

onde A é a matriz de coeficientes formada pelos termos que multiplicam $\phi_{m-1}, \phi_{m}$ e $\phi_{m+1}$ definidos na expressão Eq. 1.3.13. Dessa forma, a Equação de Helmholtz é introduzida como a equação governante de sistemas aqüíferos homogêneos e isotrópicos em multicamadas (Hemker, 1984).

De uma forma geral, as equações governantes do escoamento subterrâneo (Laplace, Poisson, Calor e Helmholtz) são utilizadas apenas em meios homogêneos e isotrópicos. No entanto, embora as imposições de homogeneidade e isotropia sejam simplificações bastante difundidas e bem aceitas, a Equação de Helmholtz é aplicada ainda em casos onde a 
condutividade do meio é dada por uma distribuição obtida da seguinte equação, onde $\chi$ é uma constante real:

$$
\nabla^{2} K^{1 / 2}+\chi^{2} K^{1 / 2}=0
$$

Georghitza (1969) demonstra que a equação governante do escoamento através de meios cuja condutividade seja assim caracterizada é escrita na seguinte forma:

$$
\nabla^{2} \varphi+\chi^{2} \varphi=0
$$

onde a variável $\varphi$ é definida como $\varphi=\phi K^{1 / 2}$. Em aqüíferos confinados $(N=0)$, a demonstração de Georghitza é aplicada a aproximações bidimensionais Eq. 1.3.3 utilizandose distribuições de transmissividade $(T=K b)$ obtidas da Eq. 1.3.15. 


\section{Capítulo 2}

\section{FERRAMENTAS ANALÍTICAS EM ÁGUAS SUBTERRÂNEAS}

Neste capítulo, é apresentada uma revisão de ferramentas matemáticas comumente utilizadas na análise de problemas de escoamento em meios porosos e em disciplinas voltadas a problemas matematicamente análogos. Na primeira seção, alguns conceitos úteis à análise de campos vetoriais são apresentados em termos de variáveis comumente utilizadas para descrever o escoamento de águas subterrâneas. Na segunda seção, são introduzidos os conceitos de variáveis complexas e funções analíticas culminando na apresentação de algumas funções harmônicas importantes na formulação do Método de Elementos Analíticos. Por fim, são apresentadas duas técnicas auxiliares bastante utilizadas na obtenção de soluções analíticas de problemas em aqüíferos alongados.

\section{1 Águas Subterrâneas e Campos Vetoriais}

A lei de Darcy é discutida por Hubbert $(1956,1969)$ em termos de uma relação entre o campo gravitacional ou campo de forças (E) e o campo de escoamento (q). Para um fluido cuja massa específica é invariável, a força que atua no fluido por unidade de massa $\mathbf{E}$ é determinada fazendo:

$$
\mathbf{E}=-g \nabla \phi
$$

onde $\phi=\frac{p}{\rho g}+z, g$ é a aceleração da gravidade $\left[\mathrm{LT}^{-2}\right], z[\mathrm{~L}]$ é a posição do ponto na direção vertical, $p\left[\mathrm{MT}^{-2} \mathrm{~L}^{-1}\right]$, a pressão do fluido no ponto e $\rho\left[\mathrm{ML}^{-3}\right]$ a massa específica do fluido.

O campo de escoamento, por sua vez, independentemente do campo de forças, deve satisfazer a conservação de massa, produzindo a Equação da Continuidade em termos do vetor de descarga de volume por unidade de área da seção transversal $\mathbf{q}\left[\mathrm{LT}^{-1}\right]$ para o fluido incompressível na forma de:

$$
\nabla \mathbf{q}=\stackrel{v}{\gamma}
$$


onde $v^{v}\left[\mathrm{~T}^{-1}\right]$ é a produção de massa fluida por unidade de volume.

Ambos, escoamento e força gravitacional, estão relacionados pela lei de Darcy da seguinte forma (Hubbert, 1956):

$$
\mathbf{q}=D \cdot \mathbf{E},
$$

encontrando analogia em diversas aplicações da física, como eletrostática e condução de calor. Definindo o fator $D$ (Eq. 2.1.3) a partir de $K$ (condutividade hidráulica) como $D=K / g$ a relação é expressa ainda como:

$$
\mathbf{q}=-K \cdot \nabla \phi
$$

onde $K$ é a condutividade hidráulica do meio, podendo ser generalizada pelo tensor $\underline{\underline{K}}$ definido na equação Eq. 1.2.5.

\subsubsection{Campos Irrotacionais}

Em casos onde o meio é isotrópico e homogêneo, o tensor $\underline{\underline{K}}$ torna-se de ordem zero e, portanto um escalar K. Dessa forma, a Eq. 2.1.4 pode ser reescrita definindo um potencial de descarga $\Phi=K \phi$, mais comumente chamado apenas de "potencial” (Bear, 1972 pág. 129) da seguinte forma:

$$
\mathbf{q}=-\nabla \Phi
$$

Assumindo esta forma, o campo de escoamento é chamado de campo conservativo ou escoamento potencial e matematicamente, $\Phi$, é tratado sob a ótica da teoria do potencial (e.g. Bear, 1972; Kellogg, 1959). A denominação de um potencial de descarga $\Phi$ em suas diversas aplicações, incluindo escoamentos em meios porosos, é feita de maneira a obter funções $\mathbf{F}$ que satisfaçam a expressões escritas na forma:

$$
\mathbf{F}=-\nabla \Phi
$$


Considerando um fluido incompressível, a equação Eq. 2.1.5 acoplada à Equação da Continuidade (Eq. 2.1.2) produz:

$$
\vec{\nabla}^{2} \Phi=-\gamma^{v}
$$

O campo caracterizado pela equação Eq. 2.1.7 é também chamado de irrotacional. Campos irrotacionais onde $\nabla \cdot \mathbf{q}=0$ são também chamados solenóides e são determinados pela Equação de Laplace:

$$
\vec{\nabla}^{2} \Phi=0
$$

A condição de irrotacionalidade, por sua vez, é definida pelo teorema de Stokes (e.g. Bear, 1972; Kellogg, 1959):

$$
\oint_{(C)} \mathbf{q} \cdot d \mathbf{s}=\iiint_{(V)} \operatorname{rot} \mathbf{q} \cdot d V=0
$$

Portanto, o campo não trivial $(\mathbf{q} \neq 0)$ que satisfaz a equação Eq. 2.1.9 é encontrado fazendo $\operatorname{rot} \mathbf{q}=0$, ou seja,

$\nabla \times \mathbf{q}=\left|\begin{array}{ccc}\hat{i} & \hat{j} & \hat{k} \\ \partial / \partial x & \partial / \partial y & \partial / \partial z \\ \mathbf{q}_{x} & \mathbf{q}_{y} & \mathbf{q}_{z}\end{array}\right|=\left(\frac{\partial \mathbf{q}_{z}}{\partial y}-\frac{\partial \mathbf{q}_{y}}{\partial z}\right) \hat{i}+\left(\frac{\partial \mathbf{q}_{x}}{\partial z}-\frac{\partial \mathbf{q}_{z}}{\partial x}\right) \hat{j}+\left(\frac{\partial \mathbf{q}_{y}}{\partial x}-\frac{\partial \mathbf{q}_{x}}{\partial y}\right) \hat{k}=0$ Eq. 2.1 .10

Para que $\frac{\partial \mathbf{q}_{z}}{\partial y}=\frac{\partial \mathbf{q}_{y}}{\partial z} ; \frac{\partial \mathbf{q}_{x}}{\partial z}=\frac{\partial \mathbf{q}_{z}}{\partial x} ; \frac{\partial \mathbf{q}_{y}}{\partial x}=\frac{\partial \mathbf{q}_{x}}{\partial y}$ é necessário que $K_{x x}=K_{y y}=K_{z z}$ e que $\nabla K=0$, o que produz igualmente a equação Eq. 2.1.5. Assim, estas propriedades são válidas somente para aqüíferos homogêneos e isotrópicos.

\subsubsection{Campos Rotacionais}


Embora a definição do potencial de descarga $\Phi$ forneça uma formulação bastante simplificada para a solução de problemas de escoamento em meios porosos, sua aplicação está fortemente atrelada à irrotacionalidade do escoamento. No entanto, a obtenção de funções chamadas linhas de corrente torna possível descrever o campo de escoamento, de maneira independente do gravitacional, em duas e três dimensões. Linhas de corrente são curvas em cada instante de tempo que são tangentes à direção da velocidade, matematicamente definidas por (Bear, 1972, pág. 224):

$$
\mathbf{q} \times d \mathbf{s}=0
$$

onde o vetor $\mathbf{s}$ indica o deslocamento ao longo da linha de corrente.

Em duas dimensões horizontais, $\mathbf{q}$ passa a ser totalizado (integrado) na espessura do aqüífero (b) e a ser referido como o vetor $\mathbf{Q}$ da seguinte forma (Bear, 1972, pág. 225):

$$
\mathbf{Q}=\int_{0}^{b}\left(q_{x}+q_{y}+q_{z}\right) d z=q_{x} b+q_{y} b=Q_{x}+Q_{y}
$$

e as linhas de corrente podem ser expressas como:

$$
d x / Q_{x}=d y / Q_{y}, \text { ou ainda, } Q_{y} d x-Q_{x} d y=0 .
$$

A função que satisfaz a equação Eq. 2.1 .13 será dada por uma $\Psi=\Psi(x, y)=$ cte ao longo de uma linha de corrente $\mathbf{s}$, onde $d \Psi / d \mathbf{s}=\frac{\partial \Psi}{\partial x} \frac{d x}{d \mathbf{s}}+\frac{\partial \Psi}{\partial y} \frac{d y}{d \mathbf{s}}=0$, se:

$$
Q_{x}=-\frac{\partial \Psi}{\partial y} \text { e } Q_{y}=\frac{\partial \Psi}{\partial x}
$$

ou ainda,

$$
\mathbf{Q}=\operatorname{rot} \Psi
$$


A Equação da Continuidade (Eq. 2.1.2), por sua vez, pode ser reescrita no plano horizontal como:

$$
\nabla \mathbf{Q}=-h^{h}
$$

onde ${ }^{h}$ é a produção de fluido sobre o plano horizontal. A partir da redução da equação Eq. 2.1.2 sobre o plano horizontal, $\Phi$ é redefinido como $\Phi=b K \phi$ e $\gamma^{v}$ em Eq. 2.1.8 é substituído por $\stackrel{h}{\gamma}$. Observando as equações Eq. 2.1.14 e Eq. 2.1.5, pode ser extraída a seguinte relação entre o potencial $\Phi$ e a função de linhas de corrente $\Psi$ conhecidas como equações (ou condições) de Cauchy-Riemann (Strack, 1989).

$$
\begin{aligned}
& \frac{\partial \Psi}{\partial y}=\frac{\partial \Phi}{\partial x} \\
& \frac{\partial \Psi}{\partial x}=-\frac{\partial \Phi}{\partial y}
\end{aligned}
$$

A definição de linhas de corrente (Eq. 2.1.13) pode ser reescrita ainda como $Q_{y} d x / d \mathbf{s}-Q_{x} d y / d \mathbf{s}=0$, donde vem $Q_{s}-Q_{s-d s}=0$, que representa a conservação de um fluido em escoamento solenóide. Fazendo o divergente na Equação da Continuidade (Eq. 2.1.2) igual a zero, escreve-se $\operatorname{div} \mathbf{Q}=0$ ou ainda, $\partial Q_{x} / \partial x+\partial Q_{y} / \partial y=0$ (Bear, 1972). Isso implica que as propriedades do escoamento rotacional, embora válidas para aqǘferos anisotrópicos e heterogêneos, não abrangem fenômenos transientes nem ocorrências de recarga.

Assim, a representação de campos rotacionais é descrita expressando-se o rotacional de $\mathbf{Q}$ diferente de zero e igual a $\beta$, ou seja:

$$
\nabla \times \mathbf{Q}=\beta
$$


O campo de escoamento será determinado por linhas de corrente $\Psi$ que satisfazem a seguinte Equação de Poisson:

$$
\nabla^{2} \Psi=\beta
$$

onde $\beta$, é o rotacional de $\mathbf{Q}$. Em campos solenóides, tem-se:

$$
\nabla^{2} \Psi=0
$$

Em três dimensões, as linhas de corrente $\psi$ são obtidas pela interseção de duas superfícies características encontradas para a equação Eq. 2.1.11 (Bear, 1972, pág. 227):

$$
\begin{aligned}
& \lambda=\lambda(x, y, z)=c t e \\
& \chi=\chi(x, y, z)=c t e
\end{aligned}
$$

A geometria das linhas de corrente, por sua vez, é descrita por uma função $\Psi$ dada por $\Psi[\lambda(x, y, z), \chi(x, y, z)]=0$. Sendo o vetor $\mathbf{q}$ (não-divergente) perpendicular à normal de ambas as superfícies no ponto de interseção,

$$
\mathbf{Q}=\nabla \lambda \times \nabla \chi
$$

Aplicações de três dimensões da formulação de um campo de escoamento nãodivergente a partir de linhas de corrente são feitas somente em casos particulares conforme discutido por Bear (1972, pág. 230).

\subsubsection{Princípio da Sobreposição}

As equações Eq. 2.1.7 e Eq. 2.1.19 são suficientes para a determinação do campo piezométrico e de escoamento de diversos problemas em meios porosos. As equações possuem coeficientes constantes e, portanto são lineares, possibilitando-se o uso da sobreposição de efeitos. O principio da sobreposição está baseado na propriedade que afirma que se $\stackrel{1}{\phi}=\stackrel{1}{\phi}(x, y, z, t)$ e $\stackrel{2}{\phi}=\stackrel{2}{\phi}(x, y, z, t)$ são duas soluções particulares de uma equação 
diferencial homogênea linear, então qualquer combinação linear de $\stackrel{1}{\phi}$ e $\stackrel{2}{\phi}, \phi=C_{1} \stackrel{1}{\phi}+C_{2} \stackrel{2}{\phi}$, é solução da equação diferencial linear homogênea. Quando se trata de uma equação diferencial linear não-homogênea, como são Eq. 2.1.7 e Eq. 2.1.19, a solução pode ser escrita na forma $\phi=\stackrel{0}{\phi}+C_{1} \stackrel{1}{\phi}+C_{2} \stackrel{2}{\phi}$, onde $\stackrel{0}{\phi}$ é uma solução e é denominada integral particular, enquanto $C_{1} \stackrel{1}{\phi}+C_{2} \stackrel{2}{\phi}$ é a solução da equação homogênea, denominada solução complementar (Bear, 1972, pág. 298).

A aplicação do princípio da sobreposição pode também ser feita no tempo. Considere, por exemplo, a seguinte equação governante:

$$
\frac{\partial}{\partial x}\left(\omega_{1} \frac{\partial \phi}{\partial x}\right)+\frac{\partial}{\partial y}\left(\omega_{2} \frac{\partial \phi}{\partial y}\right)+\frac{\partial}{\partial z}\left(\omega_{3} \frac{\partial \phi}{\partial z}\right)=\omega_{4} \frac{\partial \phi}{\partial t}
$$

onde $\omega_{i}(i=1,2,3,4)$ são coeficientes independentes de $\phi$. A condição inicial no domínio $(D)$ é $\phi=f(x, y, z)$ e no contorno $(C)$ é $\mathbf{L}(\phi)=\mathrm{g}(x, y, z)$, sendo $\mathbf{L}$ um operador diferencial.

Seja $\stackrel{1}{\phi}=\stackrel{1}{\phi}(x, y, z, t)$, a solução para o seguinte escoamento dado por

$$
\frac{\partial^{2} \phi}{\partial x^{2}}+\frac{\partial^{2} \phi}{\partial y^{2}}+\frac{\partial^{2} \phi}{\partial z^{2}}=0
$$

satisfazendo as mesmas condições de contorno. Seja também $\stackrel{2}{\phi}=\stackrel{2}{\phi}(x, y, z, t)$ a solução da equação Eq. 2.1.23 agora com condição inicial

$$
\stackrel{2}{\phi}=\mathrm{f}(x, y, z)-\mathrm{g}(x, y, z)
$$

e no contorno $\mathbf{L}(\phi)=0$, de forma que $\stackrel{1}{\phi}=\mathrm{g}(x, y, z)$.

Verifica-se que $\phi=\stackrel{1}{\phi}+\stackrel{2}{\phi}$ satisfaz a equação Eq. 2.1.23 e as condições iniciais no domínio $D$ e de contorno em $C$. Dessa forma, o problema é analisado por intermédio de um problema de escoamento permanente especificado por suas condições de contorno e um problema transiente prescrito por sua condição inicial e condição de contorno homogênea. 
Segundo Bear (1972, pág. 300) o Teorema de Duhamel permite ainda que diversas soluções $\phi=\mathrm{f}(x, y, z, \tau, t)$ de problemas com condição inicial $\phi=0$ e de contorno $\phi=\Gamma(x, y, z$, $\tau)$ sejam sobrepostas ao longo do tempo. Por exemplo, a solução do problema onde são especificadas com condição inicial $\phi=0$ e de contorno $\phi=\Gamma\left(x, y, z, t_{0}\right)$ para a equação Eq. 2.1.23 é, então, obtida utilizando-se a integral:

$$
\int_{0}^{t} \frac{\partial}{\partial t} \phi(x, y, z, \tau, t-\tau) d \tau
$$

onde $\tau$ é o tempo inicial tomado igual a zero e $t-\tau$ é o tempo final igual a $t$.

Decompondo-se, então, um problema de condição inicial $\phi=\phi(x, y, z, \tau)$ e de contorno $\phi=\Gamma(x, y, z, t)$ em dois problemas, o primeiro, especificado pela condição onde $\stackrel{1}{\phi}$ em $t=0$ vale $\mathrm{f}(x, y, z)$ e zero no contorno $(C)$, e o segundo, especificado pela condição onde $\stackrel{2}{\phi}$ é zero inicialmente $(t=0)$ e vale $\Gamma(x, y, z, t)$ no contorno, sua sobreposição, $\phi=\stackrel{1}{\phi}+\stackrel{2}{\phi}$, resolve o problema. Em outras palavras, o teorema, ou princípio de Duhamel expressa o fato de que diversas ocorrências atuando simultaneamente produzem efeitos independentes. Assim, o efeito resultante pode ser obtido pela soma dos efeitos individuais.

\subsubsection{Decomposição de Helmholtz}

O teorema de decomposição de Helmholtz permite que campos vetoriais que se anulam no infinito (como o de escoamento subterrâneo) sejam completamente determinados se conhecidos seu divergente e seu rotacional em todo o domínio. Kobe (1985) estendeu esse conceito de maneira que um campo vetorial qualquer pode ser decomposto em um campo irrotacional, porém divergente, um campo divergente-nulo, porém rotacional e um campo harmônico (solenóide).

Considerando um campo de escoamento representado por $\mathbf{Q}$, se os valores de divergência $(\gamma)$ e de rotacionalidade $(\beta)$ são conhecidos, então o campo vetorial divergentenulo pode ser representado pelo rotacional do campo escalar $\Psi$ :

$$
\stackrel{\beta}{\mathbf{Q}}=\frac{\partial \Psi}{\partial x}-\frac{\partial \Psi}{\partial y}
$$


A parte irrotacional, por sua vez é representada em termos do gradiente do escalar $\Phi$ :

$$
\stackrel{\gamma}{\mathbf{Q}}=-\left(\frac{\partial \Phi}{\partial x}+\frac{\partial \Phi}{\partial y}\right)
$$

Dessa forma, o divergente de $\stackrel{\gamma}{\mathbf{Q}}$ é o Laplaciano de $\Phi$ e é igual a $\gamma$ (Eq. 2.1.7) e o rotacional de $\stackrel{\beta}{\mathbf{Q}}$ é dado, semelhantemente, por Eq. 2.1.19. A terceira componente do campo de escoamento é harmônica $(\operatorname{div} \mathbf{Q}=\operatorname{rot} \mathbf{Q}=0)$ e pode ser representada por (Strack, 2003):

$$
\stackrel{h}{\mathbf{Q}}=-\frac{\partial \stackrel{h}{\Phi}}{\partial x}-\frac{\partial \stackrel{h}{\Phi}}{\partial y}=\frac{\partial \stackrel{h}{\Psi}}{\partial x}-\frac{\partial \stackrel{h}{\Psi}}{\partial y}
$$

Assim, o campo $\mathbf{Q}$ é determinado pela soma $\mathbf{Q}=\stackrel{\beta}{\mathbf{Q}}+\stackrel{\gamma}{\mathbf{Q}}+\stackrel{h}{\mathbf{Q}}$.

\subsection{Funções Analíticas em Águas Subterrâneas}

\subsubsection{Variáveis Complexas}

Geometricamente, um número complexo é a representação de um par de coordenadas $(x, y)$ em um plano cartesiano ou polar. O plano de coordenadas complexas definidas por:

$$
z=x+i y
$$

é chamado plano complexo $z$ e os valores de $x$ e $y$ são denominados respectivamente de coordenada real e imaginária, individualmente referidas respectivamente como:

$$
x=\mathfrak{R}(z) \text { e } y=\mathfrak{I}(z)
$$

Em termos de coordenadas polares $(r, \theta)$, um número complexo pode ser representado da seguinte forma: 


$$
z=x+i y=r(\cos \theta+i \sin \theta)
$$

Um número complexo é representado, ainda, de uma terceira maneira, conhecida como fórmula de Euler. Expandindo-se as funções seno e cosseno com a série de Taylor, encontrase:

$$
z=r e^{i \theta}=|z| e^{i \arg (z)}
$$

Dessas definições, são advindas diversas propriedades algébricas cuja discussão é encontrada, por exemplo, em Strack (1989).

\subsubsection{Funções Analíticas}

Funções complexas diferenciáveis são definidas pela verificação das condições de Cauchy-Riemann. Os campos escalares $\Phi$ e $\Psi$ são exemplos de funções que satisfazem tais condições, e portanto expressam a parte real e a imaginária de uma função complexa:

$$
\Omega=\Omega(z)=\Phi+i \Psi
$$

A função $\Omega$ é conhecida como o potencial complexo, onde a decomposição $z=x+i y$ e todas demais propriedades algébricas observadas em $z$ são válidas também para $\Omega$. O vetor de descarga $\mathbf{Q}$, por sua vez, pode ser representado por uma variável complexa $(W)$, obtida de maneira semelhante à equação Eq. 2.1.5, diferenciando-se o potencial em relação à variável de espaço (z):

$$
W=-\frac{d \Omega}{d z}=-\frac{\partial \Phi}{\partial x}-i \frac{\partial \Psi}{\partial y}
$$

O potencial $(\Phi)$ pode ser escrito em duas dimensões considerando a espessura do aqüífero $(b)$ constante a partir da lei de Darcy para meios homogêneos isotrópicos (Eq. 1.2.7), tem-se:

$$
\mathbf{Q}=\mathbf{q} b=-b \cdot K \nabla \phi
$$


Se $b$ não varia no plano $x y, \Phi$ pode ser escrito agora como $\Phi=K b \phi$ e assim

$$
\mathbf{Q}=-\nabla \Phi
$$

Utilizando as equações Eq. 2.1.14 e Eq. 2.1.5 para relacionar as coordenadas de $W$ $(\Phi$ e $\Psi)$ às componentes de $\mathbf{Q}$, tem-se:

$$
W=Q_{x}-i Q_{y}
$$

\subsubsection{Funções Harmônicas}

A Equação de Laplace é uma das formas de equação governante mais freqüentes em problemas de escoamento subterrâneo. As funções que satisfazem a Equação de Laplace são chamadas funções harmônicas e são bastante úteis na análise matemática de campos vetoriais. Além disso, o princípio da sobreposição possibilita a aplicação dessas funções à resolução de outras formas de equações diferenciais lineares. Por exemplo, escoamentos governados pela Equação de Poisson podem ser determinados pela sobreposição de funções harmônicas e de uma solução particular de Poisson.

\subsubsection{O potencial complexo}

Demonstra-se que a expressão utilizada para a definição de potencial complexo Eq. 2.2.5 satisfaz a Equação de Laplace da seguinte maneira. Sabendo que $\Phi$ e $\Psi$ são harmônicas (ver Eq. 2.1.8 e Eq. 2.1.20) e seguindo o princípio da sobreposição, a combinação linear de $\Phi$ e $\Psi$ produz também uma função harmônica:

$$
\nabla^{2} \Phi+i \nabla^{2} \Psi=\nabla^{2} \Omega=0
$$

\subsubsection{Escoamento Uniforme}

A função complexa de descarga para o escoamento uniforme com componentes $Q_{x 0} \mathrm{e}$ $Q_{y 0}$ é obtida escrevendo a função de descarga (Eq. 2.2.9) para uma dada coordenada $x_{0}, y_{0}$. 
$W_{u}=Q_{x 0}-i Q_{y 0}$

Eq. 2.2.11

Por sua vez, o potencial complexo é expresso a partir da expressão Eq. 2.2.6 por:

$$
\Omega_{u}=-\int\left(Q_{x 0}-i Q_{y 0}\right) d z=-\left(Q_{x 0}-i Q_{y 0}\right) z+\Omega_{0}
$$

onde $\Omega_{0}$ é um potencial de referência localizado em um ponto do plano também escolhido como referência.

A distribuição de potenciais de descargas devido a um escoamento uniforme é obtida da parte real de $\Omega$ :

$$
\Phi_{u}=\mathfrak{R}\left[-Q_{x 0}(x+i y)+i Q_{y 0}(x+i y)+\Omega_{0}\right]=-Q_{x 0} x-Q_{y 0} y+\Phi_{0}
$$

As linhas de corrente do campo, por sua vez, são obtidas da componente imaginária de $\Omega$

$$
\Psi_{u}=\mathfrak{I}\left[-Q_{x 0}(x+i y)+i Q_{y 0}(x+i y)+\Omega_{0}\right]=Q_{y 0} x-Q_{x 0} y+\Psi_{0}
$$

\subsubsection{Potencial Logarítmico}

O escoamento horizontal gerado por uma descarga uniformemente distribuída ao longo da parede de um cilindro (considere-se um poço de penetração completa) pode ser caracterizado simetricamente em relação ao eixo vertical desse cilindro. O campo assim descrito é determinado em coordenadas polares por:

$$
\frac{\partial \mathbf{Q}}{\partial \mathbf{r}}=0
$$

Em termos de variáveis complexas,

$$
\frac{\partial W}{\partial z}=0
$$


Considerando a origem das coordenadas $z$ localizada no centro do poço, é dado que em $r=r_{0}, \mathbf{Q}_{p}=-2 \pi r \mathbf{Q}_{r}$, sendo $\mathbf{Q}_{r}$ a descarga em qualquer ponto posicionado com distância $r$ a partir do poço, onde a descarga é $\mathbf{Q}_{p}$. Ou seja, escrevendo-se a vazão complexa $W_{r}=\left|\mathbf{Q}_{r}\right| e^{-i \theta}$ e a coordenada complexa $z=r e^{i \theta}$, tem-se:

$W_{p}=-2 \pi z W_{r}$

Assim, a partir da expressão Eq. 2.2.6, tem-se:

$W_{r}=-\frac{d \Omega}{d z}=\frac{W_{p}}{-2 \pi z}$

Integrando-se $\Omega$ em $z$, tem-se:

$\Omega_{p}=\frac{\left|W_{p}\right|}{2 \pi} \ln \left(z-z_{r}\right)+C_{p}$

O módulo de $W_{p}$ é igual à vazão bombeada no poço $(Q)$. Dessa forma, a componente real e a componente imaginária do potencial logarítmico complexo são expressas pelas seguintes funções:

$$
\begin{aligned}
& \Phi_{p}=\frac{Q}{2 \pi} \ln \left|z-z_{r}\right|+C_{p} \\
& \Psi_{p}=\frac{Q}{2 \pi} \theta
\end{aligned}
$$

Observe-se que a função $\Psi_{p}$ é descontínua em toda linha entre $\theta=\pi$ e $\theta=-\pi$ (Figura 2.2.1). O intervalo da função nessa linha é chamado ramo e fornece o bombeamento do poço (Q) substituindo-se os valores de $\theta$ na Eq. 2.2.21 e fazendo a subtração $\Psi^{+}-\Psi$. 


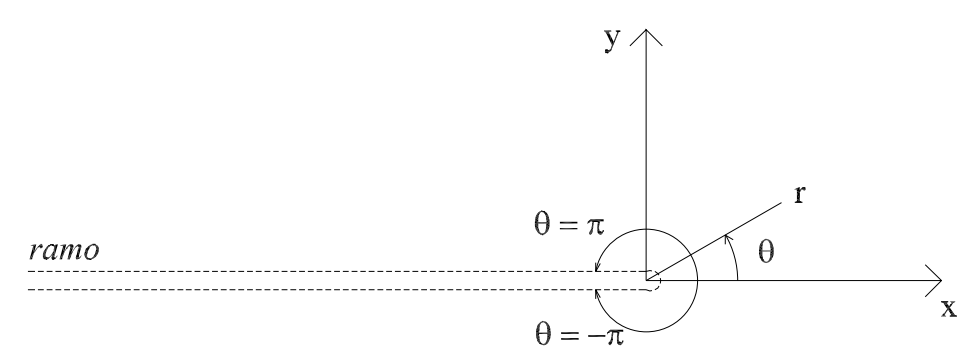

Figura 2.2.1 - Ramo no potencial logarítmico

\subsubsection{Dipolo Plano}

A função dipolo é utilizada em diversos problemas de valor de contorno. Denomina-se "dipolo plano" a função definida pelo caso limite onde dois poços com valores de vazão opostos são posicionadas em uma distância uma da outra que tende a zero. Strack (1989, pág. 245) faz uma interessante apresentação de utilização da função dipolo, deduzindo sua expressão a partir de um problema de escoamento, onde um lago circular é submetido a um campo de escoamento uniforme. O potencial devido ao "dipolo plano" $\left(\Phi_{d}\right)$ é então dado por:

$$
\Phi_{d}=\frac{1}{2 \pi} \frac{s_{x} r_{x}+s_{y} r_{y}}{|\mathbf{r}|^{2}}+C_{d}
$$

ou ainda:

$$
\Phi_{d}=\frac{|s|}{2 \pi} \frac{\cos (\theta-\beta)}{|\mathbf{r}|}+C_{d}
$$

onde $C_{d}$ é a constante de integração; s é o complexo que contém intensidade e direção do dipolo e $s_{x}$ e $s_{y}$ suas componentes real e complexa; $\theta=\arctan \frac{y-y_{0}}{x-x_{0}}$ é a orientação do vetor de posição representado por $\mathbf{r}$ e $\beta=\arg (s)$.

Utilizando-se as condições de Cauchy-Riemann (Eq. 2.1.17), obtém-se: 


$$
\Psi_{d}=-\frac{1}{2 \pi} \frac{s_{x} r_{y}-s_{y} r_{x}}{|\mathbf{r}|^{2}}
$$

ou ainda

$$
\Psi_{d}=-\frac{|s|}{2 \pi} \frac{\operatorname{sen}(\theta-\beta)}{|\mathbf{r}|}
$$

Das equações Eq. 2.2.24 e Eq. 2.2.25 obtém-se o potencial complexo devido ao dipolo expresso por:

$$
\Omega_{d}=\frac{|S|}{2 \pi|\mathbf{r}|}[\cos (\theta-\beta)-i \operatorname{sen}(\theta-\beta)]=\frac{|S|}{2 \pi|\mathbf{r}|} \frac{e^{i \beta}}{e^{i \theta}}=\frac{s}{2 \pi\left(z-z_{0}\right)}
$$

\subsubsection{Vórtice Plano}

Um vórtice plano é o escoamento rotacional gerado em torno de um único ponto. Embora a determinação do escoamento em aqüíferos sob condicionantes que o tornam rotacional em todo domínio venha sendo crescentemente abordada na literatura científica, a função vórtice apresentada a seguir pode ser aplicada somente em determinados pontos do contorno onde o potencial é descontínuo.

Parte-se do princípio de que as linhas de corrente são circulares - o escoamento é rotacional no vórtice, semelhantemente às linhas equipotenciais devido a um poço de bombeamento sob as condições de Cauchy-Riemann. Conseqüentemente, as linhas equipotenciais são radiais. Assim, podemos escrever que:

$$
\Omega_{v}=i A \ln \left(z-z_{r}\right)+\Omega_{v 0}
$$

onde $A$ é uma constante a ser determinada. A parte real e a imaginária de $\Omega_{\mathrm{v}}$ são escritas fazendo

$$
\Omega_{v}=i A \ln \left(\left|z-z_{r}\right| e^{i \theta}\right)+\Omega_{v 0}=A\left(-\theta+i \ln \left|z-z_{r}\right|\right)+\Phi_{v 0}+i \Psi_{v 0}
$$


então,

$$
\Phi_{v}=-A \theta+\Phi_{v 0}
$$

e

$$
\Psi_{v}=A \ln \left|z-z_{r}\right|+\Psi_{v 0}
$$

A constante $A$ pode ser determinada considerando a seguinte aplicação particular. Considere o escoamento produzido pela diferença de potencial devido à construção de uma barragem em um rio (Strack 1989, pág. 255). A carga piezométrica é considerada constante ao longo do rio, sendo que o potencial a montante da barragem vale $\Phi_{1}$ e a jusante, $\Phi_{2}$. Considerando-se ainda que a jusante da barragem $\theta$ vale zero e a montante vale $\pi$, tem-se

$$
A=-\frac{\Phi_{1}-\Phi_{2}}{\pi}
$$

O ramo da função vórtice $\left(\Phi_{v}\right)$ é dado entre $\theta=\pi$ e $\theta=-\pi$ e o valor de $\Phi^{+}-\Phi^{-}$é igual a $2\left(\Phi_{1}-\Phi_{2}\right)$. A função de vórtice tem sido bastante utilizada, por exemplo, em estudos de escoamentos multifásicos, onde ocorrem descontinuidades no potencial $\Phi$ na interface entre os fluidos (Haitjema, 1991; De Josselin De Jong, 1960; etc).

As quatro funções harmônicas apresentadas até aqui são desenvolvidas para o potencial na sua forma complexa $\Omega$ no plano complexo $x+i y$ e, portanto, a derivada do potencial $\Phi$ e da função de corrente $\Psi$ existe em qualquer ponto do plano. As funções harmônicas também podem ser encontradas no espaço $(x, y, z)$. No entanto, com o laplaciano de $\Omega$ restrito ao plano, são consideradas a seguir somente algumas funções obtidas do laplaciano de $\Phi$.

\subsubsection{Potencial de Newton}

Duas funções utilizadas como soluções fundamentais para a descrição de efeitos produzidos sobre campos conservativos tridimensionais são o potencial de Newton e o 
"dipolo elétrico" (em analogia ao eletromagnetismo). O potencial de Newton descreve a distribuição de potencial devido à presença de uma fonte/sumidouro pontual e é dado por:

$$
\Phi=-\frac{Q}{4 \pi r}
$$

onde $r$ é a distancia da posição da fonte ao ponto analisado e $\Phi$ é definido no espaço por $\Phi=K \phi$.

O "dipolo elétrico" apresentado a seguir, como definido na função "dipolo plano" descreve a distribuição do potencial devido ao posicionamento de duas cargas pontuais de valores opostos. No espaço tridimensional, o potencial devido à presença de um "dipolo elétrico" é apresentado em Luther (1998) como:

$$
\Phi=-\sigma \frac{\mathbf{c . r}}{4 \pi r^{3}}
$$

onde c é o vetor de orientação do dipolo; r é o vetor que une a posição do dipolo ao ponto analisado e $r$ é o comprimento do vetor $\mathbf{r}$.

\subsubsection{Potencial de Zhukovski}

Dada a Equação de Laplace no plano vertical (y,z), a função de Zhukovski (Eq. 2.2.34) é também harmônica (Bear, 1972, pág. 403) e pode ser estendida para o espaço tridimensional (Luther, 1998).

$$
\chi=\Phi-K z=\frac{p}{\rho g}
$$

onde $p$ é a pressão relativa e $\rho g$ é o peso específico do fluido. A função de Zhukovski é também conhecida como mapeamento de Zhukovski, uma vez que a superfície freática e superfície de afloramento ("seepage face") são transformadas no eixo $\chi=0$ do plano- $\chi \bar{\chi}$ (Bear, 1972).

Luther (1998) utiliza a função na especificação da condição de contorno em termos de escoamento potencial na superfície freática e de afloramento produzida por um poço de 
penetração parcial. Dessa forma, a função $\chi$ é ainda chamada potencial de Zhukovski. Considerando o caso de não haver recarga sobre o lençol de água, ou seja $\mathbf{q}_{\mathrm{n}}=0$ e $\chi=0$, temse (Luther, 1998 pág. 31):

$$
\nabla \Phi \frac{\nabla \chi}{|\nabla \chi|}=0
$$

Em casos onde considera-se recarga do lençol, Luther (1998, pág. 32) fornece:

$$
\nabla \Phi \nabla \chi=-N \nabla \chi
$$

\subsection{Integrais de Linha em Águas Subterrâneas}

As funções discutidas nesta seção são obtidas integrando-se funções de variáveis complexas sobre uma linha. As integrais de linha complexas são conhecidas pelo nome de integrais de Cauchy. Em se tratando de funções como aquelas discutidas na seção anterior (funções singulares), a integral de linha comumente recebe o nome de integrais singulares devido às aplicações propostas por Muskhelishvili, (1958).

\subsubsection{Linha Poço}

A integração da função Eq. 2.2.19 em uma linha tem importantes aplicações na representação de drenos não só para escoamentos subterrâneos (e.g. Carslaw e Jaeger, 1959; Strack, 1989). Por exemplo, considerando-se poços igualmente espaçados de $\Delta \xi$ ao longo de um segmento de linha reta no plano, como ilustrado na Figura 2.3.1, e a descarga de cada poço dada por $Q=\sigma \Delta \xi$, $\sigma$ será uma taxa de descarga uniformemente distribuída ao longo do linha. 


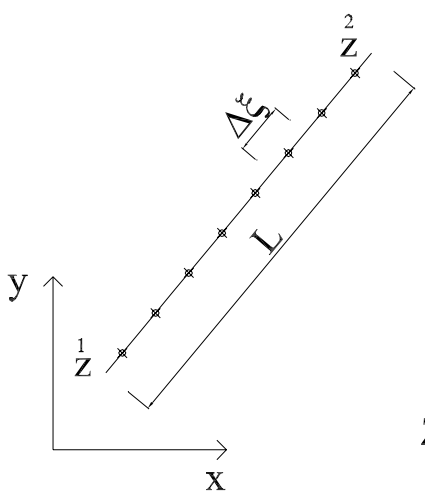

(a)

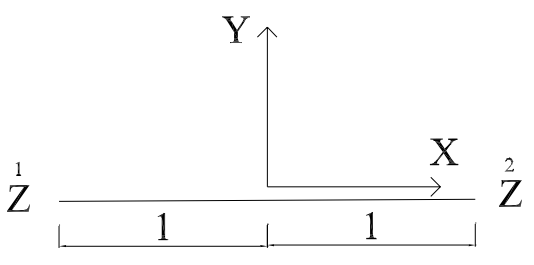

(b)

Figura 2.3.1 - Esboço para uma linha poço

Fazendo $\Delta \xi \rightarrow 0$, o potencial complexo devido à soma dos poços ao longo da linha é dado por:

$$
\Omega=\int_{-L / 2}^{L / 2} \frac{\sigma}{2 \pi} \ln (z-\delta) d \xi
$$

onde $\delta$ indica a coordenada complexa de cada poço e pode ser reescrita como:

$$
\delta=\xi e^{i \alpha}+\frac{1}{2}\left(\begin{array}{ll}
1 & 2 \\
z & z
\end{array}\right)
$$

onde $\frac{1}{2}\left(\begin{array}{cc}1 & 2 \\ Z & +Z\end{array}\right)$ corresponde ao centro do segmento e $\alpha$ é o ângulo que o segmento $z-Z$ forma com o eixo $x$ (ver Figura 2.3.2a). Adimensionalizando-se $\delta$ e $z$ em relação à metade do comprimento $\frac{1}{2}\left(\begin{array}{cc}2 & 1 \\ Z & -Z\end{array}\right)$, em $\Delta \mathrm{e} Z$, respectivamente, tem-se

$$
Z=\frac{Z-\frac{1}{2}\left(\begin{array}{c}
1 \\
Z+Z
\end{array}\right)}{\frac{1}{2}\left(\begin{array}{l}
2 \\
Z-Z
\end{array}\right)} \text { e } \Delta=\frac{\delta-\frac{1}{2}\left(\begin{array}{l}
1 \\
\delta
\end{array}+\stackrel{2}{\delta}\right)}{\frac{1}{2}\left(\begin{array}{l}
2 \\
\delta
\end{array}-\delta\right)}
$$


A definição (Eq. 2.3.3) é utilizada na construção de diversas funções empregadas no Método de Elementos Analíticos. Escrevendo-se $d \xi=e^{-i \alpha} d \delta$ com base na definição Eq. 2.3.2 e $\mathrm{e}^{-\mathrm{i} \alpha} d \delta=L d \Delta / 2$ com base na transformação Eq. 2.3.3, o potencial da linha de poços pode ser reescrito como

$$
\Omega=\int_{-1}^{1} \frac{\sigma}{2 \pi} \ln \left[\frac{1}{2}\left(\begin{array}{cc}
2 & 1 \\
Z & -Z
\end{array}\right)(Z-\Delta)\right] \frac{1}{2} L d \Delta
$$

A integral produz a seguinte função (Strack, 1989 pág. 285):

$$
\Omega=\frac{\sigma L}{4 \pi}\left\{(Z+1) \ln (Z+1)-(Z-1) \ln (Z-1)+2 \ln \left[\frac{1}{2}\left(\begin{array}{ll}
2 & 1 \\
Z & -Z
\end{array}\right)\right]-2\right\} .
$$

O potencial $\Phi$ e a descarga $Q$, podem ser expressos a partir do potencial complexo Eq. 2.3.5 utilizando-se as definições apresentadas na seção 2.2.2. O ramo da função Eq. 2.3.5 na parte imaginária $\Psi$ ocorre sobre o eixo $Y=0$ (ver Figura 2.3.2b) e é analisado a partir da expressão (Strack, 1989):

$$
\begin{aligned}
& \Psi=\frac{\sigma L}{4 \pi}\left[(X+1) \theta_{1}-(X-1) \theta_{2}+2 \alpha\right] \\
& =\frac{\sigma L}{4 \pi}\left[-X\left(\theta_{2}-\theta_{1}\right)+\theta_{1}+\theta_{2}+2 \alpha\right]
\end{aligned}
$$

Para todas as regiões onde se observa ramo, a descontinuidade na função $\Psi$ é igual à vazão $\left(Q_{\eta}^{+}-Q_{\eta}^{-}\right) L=\sigma L$ extraída na linha poço (Figura 2.3.2c):

$$
\left\{\begin{array}{lll}
-\infty<X<-1 & Y^{+}=0 & \Psi^{+}=\frac{\sigma L}{4 \pi}(2 \pi+2 \alpha) \\
-\infty<X<-1 & Y^{-}=0 & \Psi^{-}=\frac{\sigma L}{4 \pi}(-2 \pi+2 \alpha) \\
-1 \leq X \leq 1 & Y^{+}=0 & \Psi^{+}=\frac{\sigma L}{4 \pi}(-X \pi+\pi+2 \alpha) \\
-1 \leq X \leq 1 & Y^{-}=0 & \Psi^{-}=\frac{\sigma L}{4 \pi}(X \pi-\pi+2 \alpha)
\end{array}\right.
$$




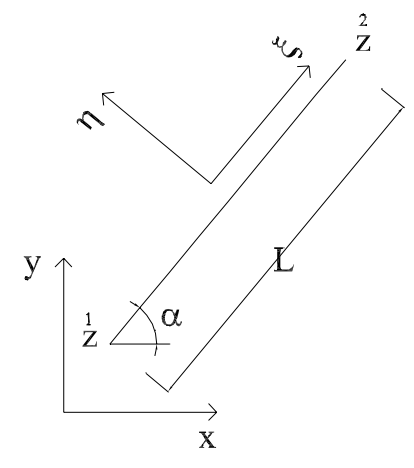

(a)

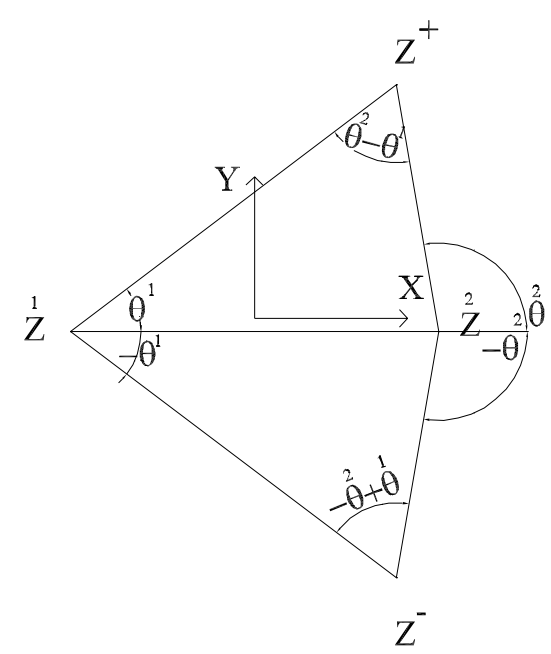

(b)

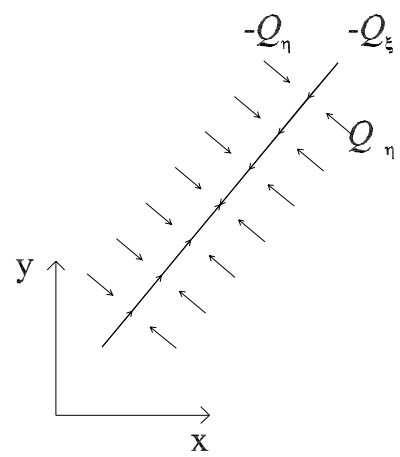

(c)

Figura 2.3.2 - Definições de ângulos e componentes de descarga para a função linha poço.

A vazão $Q_{\eta}$ é definida a partir da expressão Eq. 2.2.6 como:

$W=-\frac{d \Omega}{d \varsigma}=Q_{\xi}-i Q_{\eta}$

onde $\zeta=\xi+i \eta=z-\frac{1}{2}\left(\begin{array}{l}2 \\ z\end{array}\right)$.

\subsubsection{Linha Dipolo}

A linha dipolo é uma distribuição contínua de dipolos alinhados sobre um segmento de reta. Integrações da função dipolo ao longo de linhas têm sido amplamente utilizadas em campos irrotacionais como ocorre em aqüíferos homogêneos isotrópicos. Grande parte dos elementos utilizados no Método de Elementos Analíticos utiliza integrações da função dipolo (Strack, 1989). De maneira semelhante à linha poço, a expressão para a distribuição de dipolos sobre uma linha é obtida integrando-se a função Eq. 2.2.26 ao longo do segmento de reta, produzindo: 


$$
\Omega=-\frac{1}{2 \pi} \int_{\substack{z \\ z}}^{z} \frac{\mu e^{i \alpha}}{z-\delta} d \xi
$$

onde o esforço $s$ é representado por $-\mu$. Utilizando-se a definição (Eq. 2.3.2) a integral do dipolo torna-se:

$$
\Omega=-\frac{1}{2 \pi} \int_{\substack{1 \\ z}}^{2} \frac{\mu}{z-\delta} d \delta
$$

A expressão pode ser adimensionalizada pela definição Eq. 2.3.3, encontrando-se:

$$
\Omega=-\frac{1}{2 \pi} \int_{-1}^{1} \frac{\mu(\Delta)}{Z-\Delta} d \Delta
$$

De maneira a expressar $\mu$ como uma função da posição $Z$, a expressão é reescrita como:

$$
\Omega=-\frac{1}{2 \pi} \int_{-1}^{1} \frac{\mu(Z)}{Z-\Delta} d \Delta+\frac{1}{2 \pi} \int_{-1}^{1} \frac{\mu(Z)-\mu(\Delta)}{Z-\Delta} d \Delta
$$

onde $Z$ e $\Delta$ são independentes. Então, resolvendo a primeira integral, tem-se:

$$
\Omega=-\frac{\mu(Z)}{2 \pi} \ln \frac{Z-1}{Z+1}+\frac{1}{2 \pi} \int_{-1}^{1} \frac{\mu(Z)-\mu(\Delta)}{Z-\Delta} d \Delta .
$$

Strack (1989, pág. 292) demonstra que a integral restante na expressão é uma função analítica e que, representando-se a distribuição de $\mu(Z)$ por um polinômio,

$$
\mu(Z)=\sum_{j=0}^{n} a_{j} Z^{j}
$$

onde $a_{j}$ é um coeficiente real, sua solução também pode ser dada por meio de um polinômio escrito na forma: 


$$
p(Z)=\sum_{m=0}^{n-1} b_{m} Z^{m}
$$

Assim, a linha dipolo pode ser expressa por:

$$
\Omega=-\frac{\mu(Z)}{2 \pi} \ln \frac{Z-1}{Z+1}+q(Z)
$$

Da integral adimensional do dipolo (Eq. 2.3.11) pode-se observar que a função aproxima-se do valor $1 / Z$ para $Z \rightarrow \infty$. Portanto, expressando-se $\mu$ por um polinômio de ordem $n$ que multiplica $\ln \frac{Z-1}{Z+1} \rightarrow-2 / Z$ quando $Z \rightarrow \infty, p(Z)$ será dado sempre por um polinômio de ordem $n-1$.

Definindo $\arg \left(\frac{Z-1}{Z+1}\right)=\theta$ com $-\pi \leq \theta \leq \pi$ (Figura 2.3.2c), $\Omega$ é distribuído ao longo de ambos os lados da linha dipolo como:

$$
\begin{array}{lll}
-1 \leq X \leq 1 & Y^{+}=0 & \Omega^{+}=-\frac{\mu(X)}{2 \pi}\left[\ln \frac{X-1}{X+1}+i \pi\right]+p(X) \\
-1 \leq X \leq 1 \quad Y^{-}=0 & \Omega^{+}=-\frac{\mu(X)}{2 \pi}\left[\ln \frac{X-1}{X+1}-i \pi\right]+p(X)
\end{array}
$$

Assim, o potencial $\Phi$ e a função de corrente $\Psi$ são contínuos ao longo de $\theta=0$, mas $\Psi$ tem um intervalo de $\Psi^{+}-\Psi^{-}=\mu(X)$ entre os dois lados da linha $(-1 \leq X \leq 1$ e $Y=0)$.

\subsubsection{Integração do Potencial de Newton sobre uma Linha}

O Potencial de Newton expressa o campo devido a uma descarga em um ponto posicionado no espaço. Sua integração sobre uma linha tem sido utilizada na formulação do escoamento devido ao bombeamento de poços de penetração parcial (e.g. Haitjema, 1985). Na Figura 2.3.3 é ilustrado um segmento de reta definido por dois vetores, $p_{i}+h_{i}$ e $p_{i}-h_{i}$. Pontos definidos ao longo da linha são definidos por $\lambda h_{i}$, onde $\lambda$ assume valores entre $-1 \mathrm{e}+1$. $\mathrm{O}$ 
vetor $r_{i}$ aponta de um ponto qualquer sobre a linha para um ponto qualquer do espaço em relação ao centro da linha $\left(t_{i}=x_{i}-p_{i}\right)$.

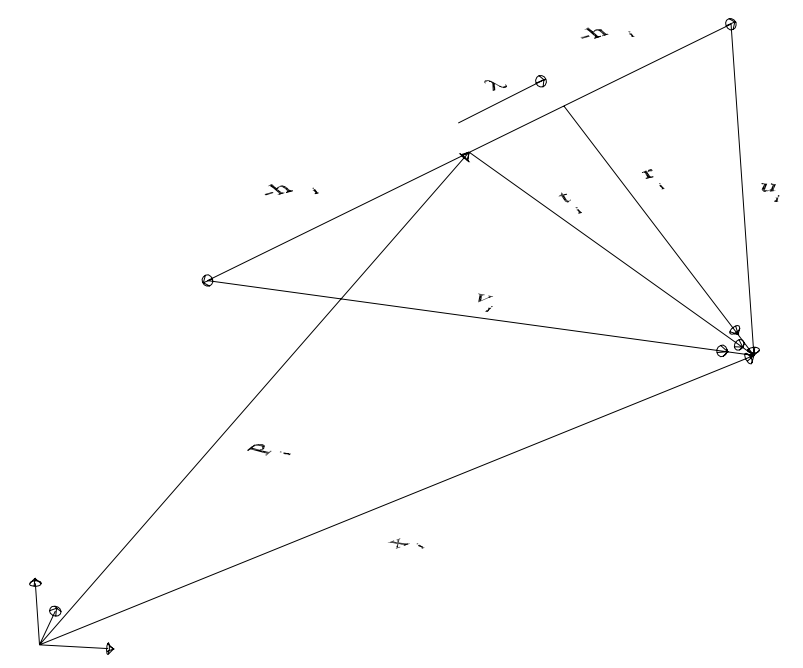

Figura 2.3.3 - Esboço para integralização de funções em uma linha no espaço.

O potencial devido a uma linha com uma distribuição de descarga polinomial $\alpha(\lambda)$ é dado por (Duschek e Hochrainer, 1954)

$$
\Phi=-\frac{1}{4 \pi} \int_{-1}^{1} \frac{\alpha(\lambda) h d \lambda}{\left[\left(t_{i}-\lambda h_{i}\right)\left(t_{i}-\lambda h_{i}\right)\right]^{1 / 2}}
$$

Haitjema (1985) apresenta uma solução para a integral expressa por Duschek e Hochrainer (1954) onde $\alpha(\lambda)$ é definido como um polinômio do terceiro grau (Eq. 2.3.19) e após desenvolvimentos adicionais utiliza a expressão obtida na representação do efeito devido ao bombeamento de poços de penetração parcial em aqüíferos confinados.

$$
\alpha(\lambda)=\alpha_{0}+\alpha_{1} \lambda+\alpha_{2} \lambda^{2}+\alpha_{3} \lambda^{3}
$$

\subsubsection{Integral do Dipolo Elétrico sobre uma Linha}

Duas linhas de dipolos ortogonais (linedoublets) são posicionadas paralelamente ao eixo do poço a fim de representar a passagem do escoamento através do poço (Haitjema, 1985). O 
potencial devido a uma linha de dipolos (Eq. 2.2.33) agrupados transversalmente é dado em Haitjema (1982 apud Haitjema, 1985) ${ }^{5}$ com distribuição de esforços $(\sigma)$ constante ao longo da linha.

$$
\Phi=\frac{\sigma}{4 \pi} \frac{2 c_{i} t_{i}\left(\frac{u_{i}+v_{i}}{2 h_{i}}\right)}{\left[\left(\frac{u_{i}+v_{i}}{2 h_{i}}\right)^{2}-1\right] u_{i} v_{i}}
$$

\subsubsection{Integração do Potencial de Newton sobre um Anel}

Funções obtidas da integração do potencial de Newton sobre uma circunferência (Figura 2.3.4) têm sido utilizadas na especificação de condições de contorno tridimensionais em escoamentos subterrâneos, como a determinação da altura da superfície freática devido ao bombeamento de poços de penetração parcial (e.g. Haitjema, 1991 e Luther, 1998).

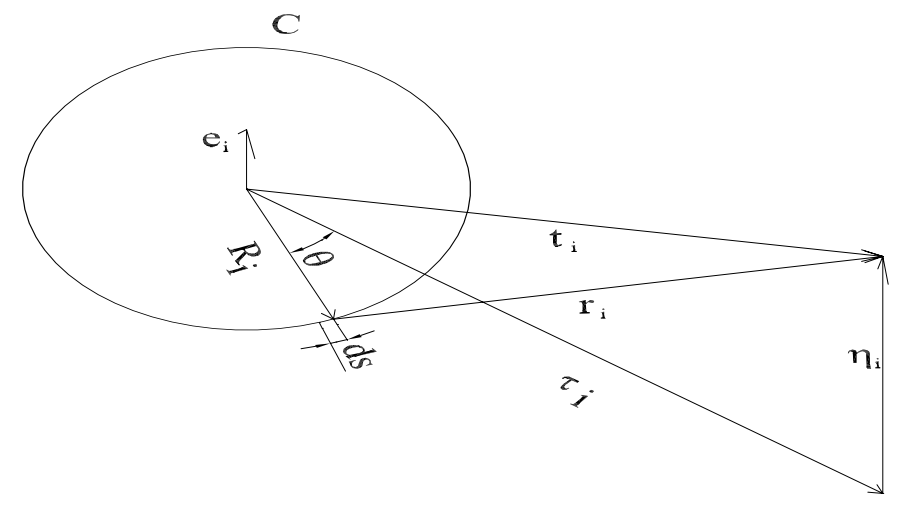

Figura 2.3.4 - Esboço de integralização de funções sobre um anel

O potencial devido a uma circunferência de descarga constante pode ser escrito por

${ }^{5}$ Haitjema, H.M. (1982) Modeling Three-Dimensional Flow in Confined Aquifers Using Distributed Singularities, tese $(\mathrm{PhD})$, University of Minnesota 


$$
\Phi=-\frac{\sigma}{4 \pi} \oint \frac{d s}{\mathbf{r}}
$$

Duschek e Hochrainer (1954) obtêm a seguinte solução para a integral:

$$
\Phi=-\frac{\sigma}{2 \pi} \sqrt{\frac{R}{\tau}} k K(k)
$$

onde $K(k)$ é a integral elíptica na sua forma completa de primeiro tipo com módulo $k$ dado por:

$$
k=\sqrt{\frac{4 \tau R}{t^{2}+R^{2}+2 \tau R}}
$$

\subsubsection{Vórtice sobre um Anel}

O potencial devido a distribuições de vórtices tem sido igualmente útil na determinação de escoamentos subterrâneos. A aplicação foi inicialmente proposta na determinação do escoamento de interface entre fluidos imiscíveis por De Josselin De Jong (1960). O potencial devido à distribuição de vórtices ao longo de uma circunferência é dado por:

$$
\Phi=\frac{\sigma}{4 \pi} \omega
$$

onde $\omega$ é o ângulo sólido que compreende a circunferência a partir do ponto onde o potencial é avaliado. Haitjema (1987) apresenta a seguinte expressão para a avaliação de $\omega$ :

$$
\varpi=\frac{2 \eta_{i} e_{i}}{\sqrt{t^{2}+R^{2}+2 \tau R}}\left[\frac{\tau-R}{\tau+R} \prod\left(\alpha^{2}, R\right)-K(k)\right]+\widetilde{\omega}(\tau \neq R)
$$

onde $\widetilde{\omega}$ é zero quando a projeção do ponto em questão sobre o plano da circunferência cai do lado de fora da mesma e $2 \pi$ quando cai dentro com o vetor $e_{i}$ apontando para fora. 
Finalmente, o valor $-2 \pi$ é encontrado quando o vetor aponta para dentro. A função $\prod\left(\alpha^{2}, R\right)$ é a integral elíptica completa do terceiro tipo com $\alpha^{2}$ definido como

$$
\begin{aligned}
& \alpha^{2}=\frac{4 \tau R}{\tau^{2}+R^{2}+2 \tau R} \\
& \text { Para } \tau=R, \\
& \omega=-\frac{\eta_{i} e_{i}}{\eta}\left[2 k^{\prime} K(k)-\pi\right]
\end{aligned}
$$

\subsection{Representação de Fronteiras Externas em Águas Subterrâneas}

\subsubsection{Método de Imagens}

O Método de Imagens é uma técnica para a especificação de condições de contorno em fronteiras externas infinitas. O método é uma aplicação do princípio da sobreposição de efeitos capaz de produzir funções pares e ímpares e é discutido na seção 4.1 de forma mais abrangente. Uma função $f$ definida em um domínio semi-infinito, por exemplo, $y<0$ é par em relação ao seu domínio complementar, $y>0$, quando $f(x, y, z)=f(x,-y, z)$ e sua derivada parcial em relação à direção de simetria, nesse caso, o eixo $y$ é igual a zero ao longo do plano de simetria $(y=0)$ (Bear, 1972). Essa definição pode ser expressa como:

$$
\begin{gathered}
\frac{\partial f(x, y, z)}{\partial x}=\frac{\partial f(x,-y, z)}{\partial x}, \frac{\partial f(x, y, z)}{\partial z}=\frac{\partial f(x,-y, z)}{\partial z} \mathrm{e} \\
\frac{\partial f(x, y, z)}{\partial y}=-\frac{\partial f(x,-y, z)}{\partial y}
\end{gathered}
$$

Substituindo $y=0$ nas expressões acima, tem-se que num mesmo ponto, $\partial f / \partial y=-\partial f / \partial y$, se $\partial f / \partial y=0$. Em problemas de valor de contorno, o enunciado feito acima pode ser empregado na especificação da condição de Neuman (onde $\partial f / \partial \eta$ é conhecido no contorno) para a determinação de funções que satisfazem equações lineares. 
Essa técnica é baseada no princípio da sobreposição de efeitos e é conhecida em diversas aplicações como Método de Imagens para o problema da barreira não-condutora (ou impermeável) infinita.

Se uma função $f$ é agora definida em $y<0$ como $f(x, y, z)=-f(x,-y, z)$, temos uma imagem impar de $f$ em $y>0$, logo,

$$
\begin{gathered}
\frac{\partial f(x, y, z)}{\partial x}=-\frac{\partial f(x,-y, z)}{\partial x}, \frac{\partial f(x, y, z)}{\partial z}=-\frac{\partial f(x,-y, z)}{\partial z} \mathrm{e} \\
\frac{\partial f(x, y, z)}{\partial y}=\frac{\partial f(x,-y, z)}{\partial y}
\end{gathered}
$$

Observando-se as condições de simetria expressas acima, o valor de $f$ no plano de simetria será o valor médio entre $f$ e $-f$ e, portanto, $f(x, 0, z)=0$. Portanto, tem-se um valor conhecido para a função ao longo de seu contorno, especificando-se condições do tipo Dirichlet por meio de sobreposições. A aplicação do Método de Imagens por meio de funções inversas é bastante difundida na determinação de campos sujeitos a uma fronteira infinita onde seu potencial é conhecido.

Os conceitos e expressões apresentadas acima permitem que o Método de Imagens seja aplicado com a mesma abrangência que o princípio da sobreposição, portanto a qualquer equação linear. Outras formas simétricas e especificações de valor de contorno obtidas por meio do método são discutidas na seção 4.1.

\subsubsection{Transformações de Schwarz-Christoffel}

Em contornos cuja geometria pode ser representada por polígonos (simples ou multiconectados) a especificação do escoamento pode ser feita nos contornos mediante o mapeamento de Schwarz-Christoffel. O mapeamento de Schwarz-Christoffel transforma um semi-plano $\zeta \geq 0 \quad(\zeta=\xi+i \eta)$ em um domínio contornado por um polígono no plano-z de modo que os pontos $\xi_{1}, \xi_{2}, \ldots, \xi_{\mathrm{n}-1}$ do eixo real no plano- $\zeta$ correspondem aos vértices $z_{1}, z_{2}, \ldots$, $Z_{\mathrm{n}-1}$ do polígono (Figura 2.4.1). 

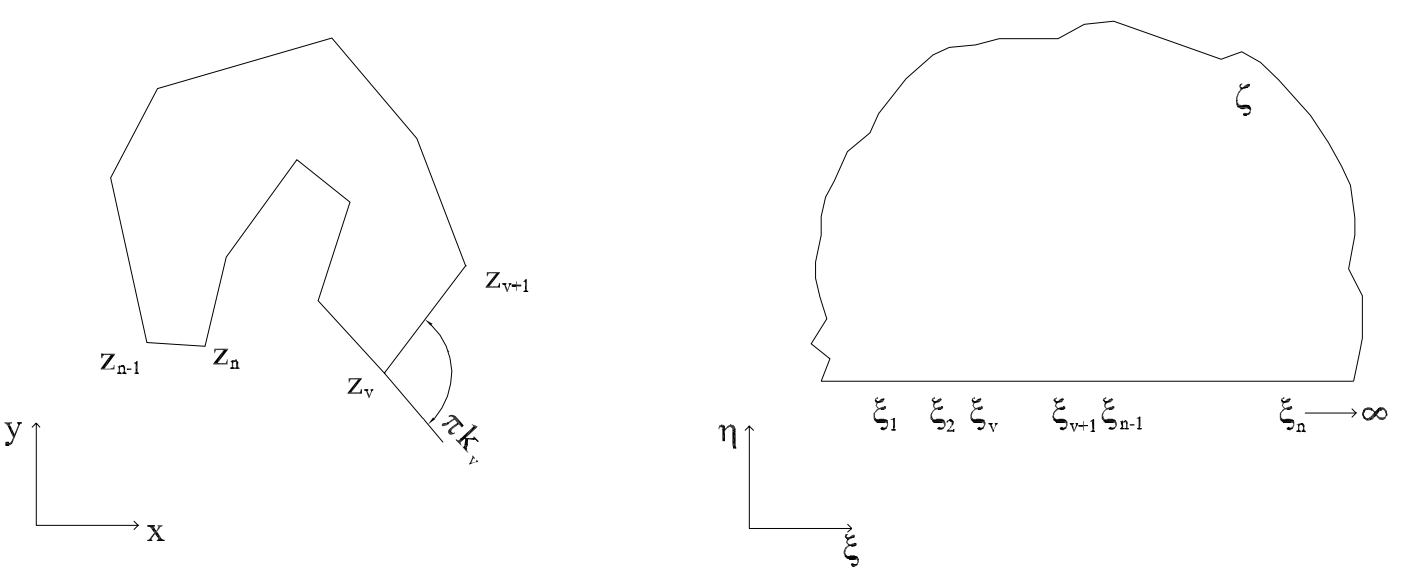

Figura 2.4.1 - Esboço do mapeamento de Schwarz-Christoffel

$O$ vértice $Z_{n}$ corresponde ao infinito no plano- $\zeta$. Um polígono pode ser representado no plano-z como:

$$
\arg (d z)_{v}-\arg (d z)_{v-1}=\pi k_{v}(v=2, \ldots, n)
$$

onde o termo $(d z)_{v}$ representa o incremento de $z$ ao longo do lado $v$ do polígono. A equação significa que $d z$ deve ser constante ao longo de cada lado do polígono, mas aumenta abruptamente de $\pi k_{v}$ se $z$ passar do vértice $z_{v}$, percorrendo-se o contorno na direção dos maiores índices, ou seja, com o domínio à esquerda. A expressão acima pode ser escrita também para o plano $\zeta$ fazendo que $\zeta$ percorra o contorno do semi-plano, o eixo real, à medida que $z$ percorre os vértices do polígono dado. Observando-se que o argumento de $d \zeta$ se mantém nulo, o argumento de $d z / d \zeta$ que é igual a $\arg (d z)-\arg (d \zeta)$ torna-se $\arg (d z / d \zeta)=\arg (d z), \operatorname{assim}:$

$$
\arg \left(\frac{d z}{d \zeta}\right)_{v}-\arg \left(\frac{d z}{d \zeta}\right)_{v-1}=\pi k_{v} \quad(v=2, \ldots, n)
$$

Introduzindo-se uma função complexa $(\chi)$ cuja parte imaginária seja dada por $\arg (d z / d \zeta)$

$$
\chi=\tau+i \omega=\ln (d z / d \zeta)
$$


pode-se escrever:

$$
\omega_{v}-\omega_{v-1}=\pi k_{v} \quad(v=2, \ldots, n)
$$

Strack (1989, pág. 372), faz analogia da condição de contorno $\omega$ com a função de corrente de poços posicionados nos vértices de um polígono cuja soma das descargas é $-2 \pi k_{v}$ para chegar à expressão de $\chi$ como:

$$
\chi=\sum_{v=1}^{n-1}-k_{v} \ln \left(\zeta-\xi_{v}\right)+C
$$

onde $C$ é uma constante complexa. Escrevendo-se a constante $C$ como $\ln A$, onde $A$ é o número complexo $|\mathrm{A}| \exp (\operatorname{iarg} \mathrm{A})$, representa-se sua parte real e imaginária da seguinte forma:

$$
\mathrm{C}=\ln A=|\arg (A)|+\mathfrak{I} \mathrm{C}
$$

Observando-se que o argumento de $\zeta-\xi_{v}$ é anulado ao longo do eixo real $\left(\xi_{n-1}<\xi<\infty\right.$ e $\eta=0$ ) a expressão Eq. 2.4.7 é escrita neste eixo como $\chi=\arg A$ e a partir de da definição Eq. 2.4.5 tem-se:

$$
\arg A=\arg \left(\frac{d z}{d \zeta}\right) \quad\left(\xi_{n-1}<\xi<\infty \text { e } \eta=0\right)
$$

Fazendo $\varphi=\arg (A)$, a função $\chi$ pode ser reescrita como:

$$
\chi=\ln \left[|A| e^{i \varphi} \prod_{v=1}^{n-1}\left(\zeta-\xi_{v}\right)^{-k_{v}}\right]
$$

ou, ainda, escrevendo $\chi=\ln (d z / d \zeta)$ :

$$
\frac{d z}{d \zeta}=|A| e^{i \varphi} \prod_{v=1}^{n-1}\left(\zeta-\xi_{v}\right)^{-k_{v}}
$$


Assim, z é obtida da seguinte integral, chamada mapeamento de Schwarz-Christoffel:

$$
Z=|A| e^{i \varphi} \int \prod_{v=1}^{n-1}\left(\zeta-\xi_{v}\right)^{-k_{v}} d \zeta+B
$$

Em casos onde o $n$-ésimo ponto $z_{v}$ mapeia um valor finito de $\xi$, o produtório estende-se até o índice $n$. 


\section{Capítulo 3}

\section{MÉTODO DE ELEMENTOS ANALÍTICOS}

Neste capítulo, o Método de Elementos Analíticos (da literatura inglesa, Analytic Element Method) é apresentado formalmente. Apenas problemas de escoamentos em aqüíferos de uma única camada são abordados por intermédio da formulação de elementos estacionários (Strack, 1989) e transientes (Zaadnoordijk, 1988). Embora outras formulações tenham sido desenvolvidas mais recentemente (e.g. Jancović, 1997; Furman e Neuman, 2003, 2004), a formulação de Strack (1989) e de Zaadnoordijk (1988) encontra-se disponível no programa $\operatorname{Tim}^{\mathrm{SL}} 0.3$ (Bakker et al., 2002) sob os termos da licença LGPL versão 2.1 (FSF, 1999).

O fundamento da formulação do Método está no princípio da sobreposição de efeitos (seção 2.1.3). Isso implica que o equacionamento governante no problema abordado deve ser linear. Com esse ponto de partida, o objetivo do método é sobrepor soluções, denominadas “elementos", para a construção de modelos realistas. Entenda-se por modelo a representação matemática de processos físicos, e.g. Mecânica dos Fluidos, Eletromagnetismo, Eletrostática, Resistência dos Materiais etc. Modelos realistas são, por sua vez, aplicações de modelos matemáticos em casos concretos produzindo respostas em formatos compreensíveis para a área de aplicação.

Em diversos campos de aplicação, problemas concretos (não hipotéticos) tornam-se complexos geometricamente e fisicamente e, por conseguinte, em sua representação matemática. A representação matemática tornando-se cada vez mais especializada, sua análise passa a ser assistida por métodos computacionais, sejam numéricos, em sua grande maioria, sejam simbólicos (computação simbólica). Embora a computação simbólica auxilie a obtenção de expressões analíticas, a complexidade geométrica de problemas concretos não permite o simples uso desses métodos como técnica de modelagem. Métodos numéricos, por sua vez, permitem uma ampla capacidade de representações espaciais, no entanto exigem a definição do domínio dentro de regiões fechadas sendo, por vezes, necessário introduzir-se contornos artificiais.

$\mathrm{O}$ Método de Elementos Analíticos oferece realismo ao modelo à medida que a necessidade de se introduzir contornos artificiais ao domínio é eliminada. O Método consiste da especificação de condições de contorno de campos vetoriais pelo princípio da sobreposição 
(Strack, 2003). Para tanto, soluções algébricas de problemas elementares descritos em termos de $\Phi, \Psi$ ou $Q$ são utilizadas como elementos do somatório (sobreposição), que expressa a condição de contorno desejada. Qualquer função obtida de uma equação governante linear pode ser empregada pelo método. Os elementos analíticos, a que se refere o nome do método, são definidos por essas funções. Embora os fundamentos e a formulação do método tenham aplicabilidade em diversas engenharias, sua programação computacional e sua aplicação tem estado voltada exclusivamente para a determinação de campos de escoamento subterrâneos.

A seguir são apresentadas algumas funções, bem como suas aplicações. Primeiramente, é definida a relação entre o potencial de descarga $\Phi$ e a altura de água $\phi$. Em seguida, são discutidos os principais elementos, estacionários e transientes, utilizados no método. Adicionalmente, são introduzidos aspectos computacionais, observados neste trabalho, para a correção de comportamentos indesejados na formulação utilizada. São apresentadas, ainda, as expressões mais comumente utilizadas em condições de contorno são apresentadas e, por fim, a implementação computacional do método.

\subsection{Potencial Unificado}

Em escoamentos subterrâneos, as equações governantes (seção 1.3) são comumente escritas em termos da carga piezométrica $\phi$ por expressões distintas para aqüíferos confinados e livres. Como discutido na seção 2.1 a lei de Darcy relaciona vazão específica (q) e carga $\phi$ por uma expressão semelhante à definição Eq. 2.1.6. Strack (e.g. 1981, 1989) reescreve equações governantes de escoamentos subterrâneos utilizando a definição Eq. 2.1.6 em termos de um potencial $\Phi$ em duas dimensões. A expressão para o potencial, válida tanto para aqüíferos livres como para aqüíferos confinados, é obtida a partir da regra de identidade de Leibnitz, expressa com a notação de Einstein, da seguinte maneira:

$$
\frac{\partial}{\partial x_{i}} \int_{0}^{h} \phi\left(x_{1}, x_{2}, x_{3}\right) d x_{3}=\int_{0}^{h} \frac{\partial}{\partial x_{i}} \phi\left(x_{1}, x_{2}, x_{3}\right) d x_{3}+\phi\left(x_{1}, x_{2}, h\right) \frac{\partial h}{\partial x_{i}}
$$

onde a variável $x$ de índice $i=1,2,3$ refere-se aos eixos três eixos cartesianos (notação de Einstein). Isolando-se o primeiro termo do segundo membro e fazendo $\phi\left(x_{1}, x_{2}, h\right)=h$, sendo $h$ a espessura, tem-se: 


$$
\int_{0}^{h} \frac{\partial}{\partial x_{i}} \phi\left(x_{1}, x_{2}, x_{3}\right) d x_{3}=\frac{\partial}{\partial x_{i}} \int_{0}^{h} \phi\left(x_{1}, x_{2}, x_{3}\right) d x_{3}-h \frac{\partial h}{\partial x_{i}}
$$

Assim, a partir da lei de Darcy, o vetor de vazão pode ser totalizado na seção do escoamento em um aqüífero homogêneo como:

$$
Q_{i}=\int_{0}^{h} \mathbf{q} d x_{3}=K \int_{0}^{h} \frac{\partial \phi}{\partial x_{i}}=K \frac{\partial}{\partial x_{i}}\left[\int_{0}^{h} \phi\left(x_{1}, x_{2}, x_{3}\right) d x_{3}-\frac{h^{2}}{2}\right]
$$

O potencial de descarga, por sua vez, torna-se definido por:

$$
\Phi=K \int_{0}^{h} \phi\left(x_{1}, x_{2}, x_{3}\right) d x_{3}-\frac{K h^{2}}{2}
$$

Substituindo-se $\phi\left(x_{1}, x_{2}, x_{3}\right)$ por sua média, $\bar{\phi}\left(x_{1}, x_{2}\right)$, obtém-se que $\int_{0}^{h} \phi\left(x_{1}, x_{2}, x_{3}\right) d x_{3}=\bar{\phi}\left(x_{1}, x_{2}\right) h$. Assim, a definição acima (Eq. 3.1.4) não utiliza as hipóteses de Dupuit (seção 1.3) para a formulação do escoamento em duas dimensões e, portanto, não foram feitas simplificações até o resultado apresentado. Utilizando-se agora as hipóteses de Dupuit, onde $\phi=h$, tem-se:

$$
\begin{array}{ll}
\Phi=K H h-\frac{K H^{2}}{2}, & \text { aqüíferos confinados com espessura } H \\
\Phi=K \frac{h^{2}}{2}, & \text { aqüíferos livres com espessura } h
\end{array}
$$

O potencial assim definido é chamado potencial unificado assumindo a expressão Eq. 3.1.5a para valores de $h \geq H$ e a expressão Eq. 3.1.5b para valores de $h<H$. Substituindo-se as relações acima nas equações Eq. 1.3 .9 e Eq. 1.3.10, por exemplo, o escoamento é governado por uma única equação:

$$
\frac{\partial^{2} \Phi}{\partial x^{2}}+\frac{\partial^{2} \Phi}{\partial y^{2}}=-N
$$


A definição pode ser estendida para um número arbitrário de camadas, separadas por camadas impermeáveis (ver Strack, 1989 pág. 115 e 492, Strack 1981a e Strack e Haitjema, 1981).

\subsection{Elementos Estacionários}

A formulação aqui discutida refere-se à metodologia apresentada em Strack (1989). Os elementos estacionários são escritos a partir de integrais singulares de Cauchy com polinômios de densidade de esforços de segunda ordem ao longo do elemento e invariáveis no tempo. São apresentados a seguir soluções elementares para o potencial devido a: um lago circular; um linesink de ordem dois; um lago poligonal; um linedipole/linedoublet de ordem dois, onde aspectos indesejados são tratados; e um areasink poligonal. Trabalhos recentes desenvolvem elementos baseados em polinômios de Chebyshev para ordens maiores que dois chamados high order elements (elementos de ordem elevada) a partir da metodologia desenvolvida em Janković (1997).

\subsubsection{Potencial de Descarga Devido a um Lago Circular}

Em uma situação hipotética, onde os gradientes encontrados no aqüífero são produzidos apenas por um lago circular, o modelo do escoamento poderia ser obtido utilizando-se a função que representa um poço de raio igual ao do lago em questão. No entanto, se o escoamento tem a influência de elementos vizinhos, a utilização da função de poço faz com que a borda do mesmo se torne diferente da equipotencial esperada para o contorno do lago. Strack (1981b) mostra que em um escoamento dado por um campo de gradientes uniformes em uma direção, a função dipolo (Eq. 2.2.26) pode ser utilizada para produzir uma equipotencial circular de centro no dipolo. Dessa forma, a combinação do bombeamento de um poço concêntrico ao lago à afixação de uma linha equipotencial sobre a borda do lago por meio da função dipolo, pode ser utilizada na modelagem de um lago circular sujeito a um escoamento regional.

Em um problema onde os gradientes piezométricos são desconhecidos, a intensidade e a direção do dipolo utilizado deverão ser obtidas de acordo com as condições de contorno especificadas para o modelo. No problema aqui idealizado, a primeira condição de contorno é obtida considerando-se que os gradientes uniformes são obtidos de medidas em qualquer 
região do aqüífero onde sua direção se mantém constante. Portanto, $Q_{x}$ e $Q_{y}$ da expressão Eq. 2.2.13 são conhecidos:

$$
\Phi_{u}=-Q_{x 0} x-Q_{y 0} y+C_{u}
$$

A segunda condição de contorno impõe que uma linha equipotencial seja mantida sobre a borda do lago e pode ser expressa utilizando-se a função dipolo Eq. 2.2.22:

$$
\Phi_{d}=\frac{1}{2 \pi} \frac{s_{x} r_{x}+s_{y} r_{y}}{|\mathbf{r}|^{2}}+C_{d}
$$

A terceira condição de contorno é obtida pelo balanço hídrico do lago. O efeito de bombeamento, ou de recarga do lago é obtido pela função de poço (Eq. 2.2.20):

$$
\Phi_{p}=\frac{Q}{2 \pi} \ln \left|z-z_{r}\right|+C_{p}
$$

Observe que qualquer função harmônica apresentada na seção 2.2.3, incluindo aquelas utilizadas neste modelo, pode ser escrita como o produto do(s) parâmetro(s) da distribuição de esforços, $\lambda_{(\mathrm{j})}$, por sua(s) função(ões) de influência, $\Lambda_{(j)}(\mathbf{r})$. Referindo-se a uma função harmônica qualquer como $\mathrm{Ha}$, pode-se escrever:

$$
H a=\lambda_{(j)} \cdot \Lambda_{(j)}(\mathbf{r})
$$

Tome-se como exemplo o caso da função dipolo Eq. 3.2.2:

$$
\Phi_{d}=\lambda_{1} \Lambda_{1}+\lambda_{2} \Lambda_{2}=s_{x} \frac{r_{x}}{2 \pi|\mathbf{r}|^{2}}+s_{y} \frac{r_{y}}{2 \pi|\mathbf{r}|^{2}}
$$

Assim a expressão Eq. 3.2.2 é escrita na forma da equação Eq. 3.2.4 separando-se os termos de acordo com os parâmetros presentes na função. A representação do escoamento proposto no problema é, então, obtida pela sobreposição dos três efeitos acima (Eq. 3.2.1, Eq. 3.2.2 e Eq. 3.2.3) da seguinte forma: 


$$
\Phi=\left[-Q_{x 0} x-Q_{y 0} y\right]+\left[\frac{1}{2 \pi} \frac{s_{x} r_{x}+s_{y} r_{y}}{|\mathbf{r}|^{2}}\right]+\left[\frac{Q}{2 \pi} \ln \left|z-z_{r}\right|\right]+C_{T}
$$

Adotando o procedimento descrito para a obtenção da função Eq. 3.2.5 tem-se para a solução do problema (Eq. 3.2.6):

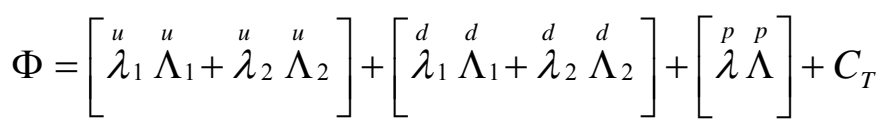

A constante $C_{T}$ é a soma das constantes de integração presentes em cada função $(C)$. Os sobrescritos presentes na expressão acima $(u, d$ e $p)$ fazem correspondência às expressões Eq. 3.2.1, Eq. 3.2.2 e Eq. 3.2.3. As funções de influência $\Lambda_{(j)}$ e os parâmetros $\lambda_{(j)}$ são ordenadas pelo índice $j$ de acordo com a posição de cada parâmetro $(j=1,2, \ldots, n)$ presente em cada expressão.

Dados os valores de vazão no lago $(Q)$, componentes do escoamento regional preferencial $\left(Q_{x}\right.$ e $\left.Q_{y}\right)$ e o valor do potencial no ponto de referência $\left(\Phi_{0}\right)$, resta determinar as componentes do esforço na função dipolo e o potencial na borda do lago $\left(\Phi_{\text {lago }}\right)$. A determinação das incógnitas do problema é feita indicando-se no domínio (no aqüífero) pontos onde o potencial pode ser especificado. Considere-se que o nível de água seja conhecido no lago e igual a $\phi_{\text {lago }}$ e deseja-se determinar seu balanço hídrico $\left(Q_{\text {lago }}\right)$. O nível de água no lago corresponde à equipotencial $\Phi_{\text {lago }}$, que por sua vez, é obtido da expressão Eq. 3.1.5a. Tomando-se três pontos da borda do lago e um quarto ponto, afastado do lago, como o ponto de referência, tem-se:

$$
\begin{aligned}
& C+\stackrel{d}{\lambda} \stackrel{d}{\Lambda}_{1}\left(z_{0}\right)+\stackrel{d}{\lambda} \stackrel{d}{\Lambda}_{2}\left(z_{0}\right)+Q_{\text {lago }} \stackrel{p}{\Lambda}\left(z_{0}\right)=\Phi_{0}-0 \\
& C+\stackrel{d}{\lambda} \stackrel{d}{\Lambda}_{1}^{\Lambda}\left(z_{1}\right)+\stackrel{d}{\lambda}_{2} \stackrel{d}{\Lambda}_{2}\left(z_{1}\right)+Q_{\text {lago }} \stackrel{p}{\Lambda}\left(z_{1}\right)=\Phi_{\text {lago }}+Q_{x} \stackrel{u}{\Lambda}_{1}\left(z_{1}\right)+Q_{y} \stackrel{u}{\Lambda}_{2}\left(z_{1}\right) \quad \text { Eq. 3.2.8 } \\
& C+\stackrel{d}{\lambda} \stackrel{d}{\Lambda}_{1}\left(z_{2}\right)+\stackrel{d}{\lambda} \stackrel{d}{\Lambda}_{2}\left(z_{2}\right)+Q_{\text {lago }} \stackrel{p}{\Lambda}\left(z_{2}\right)=\Phi_{\text {lago }}+Q_{x} \stackrel{u}{\Lambda}_{1}\left(z_{2}\right)+Q_{y} \stackrel{u}{\Lambda}_{2}\left(z_{2}\right) \\
& C+\stackrel{d}{\lambda_{1}} \stackrel{d}{\Lambda}{ }_{1}\left(z_{3}\right)+\stackrel{d}{\lambda}_{2} \stackrel{d}{\Lambda}_{2}\left(z_{3}\right)+Q_{\text {lago }} \stackrel{p}{\Lambda}\left(z_{3}\right)=\Phi_{\text {lago }}+Q_{x} \stackrel{u}{\Lambda}_{1}\left(z_{3}\right)+Q_{y} \stackrel{u}{\Lambda}{ }_{2}\left(z_{3}\right)
\end{aligned}
$$

Observe que na primeira linha do sistema, o potencial ( $\left.\Phi_{0}\right)$ é especificado em um ponto de referência $z_{0}$ e por isso é chamado potencial de referência. Nas linhas seguintes, o potencial 
é especificado com valores iguais a $\Phi_{\text {lago }}$ para os três pontos da borda do lago: $z_{1}, z_{2}$ e $z_{3}$. Do conjunto de equações Eq. 3.2.8, as incógnitas $\stackrel{d}{\lambda}{ }_{1}, \stackrel{d}{\lambda}{ }_{2}, Q_{\text {lago }}$ e $C_{T}$ podem ser determinadas.

\subsubsection{Linesinks de Ordem Dois}

O potencial devido a uma linha reta de descarga uniforme é dado pela equação Eq. 2.3.5. A ordem do elemento é definida pelo maior expoente do polinômio utilizado na representação da descontinuidade encontrada no potencial complexo (Eq. 2.2.5) (Strack, 1989). Na função Eq. 2.3.5, $\sigma$ é dado por uma constante que representa a diferença das componentes normais de descarga entre os dois lados da linesink, da seguinte forma:

$$
\sigma=-\left(Q_{n}^{+}-Q_{n}^{-}\right)
$$

Aplicando-se a definição Eq. 2.2.6 a $\sigma$, onde $\mathrm{Q}_{\eta}$ é a parte imaginária de $W$, tem-se:

$$
\sigma=-\left(Q_{\eta}^{+}-Q_{\eta}^{-}\right)=-\left.\mathfrak{J}\left\{\frac{d \Omega}{\partial \zeta}\right\}\right|_{\eta^{+}}+\left.\mathfrak{T}\left\{\frac{d \Omega}{\partial \zeta}\right\}\right|_{\eta^{-}}
$$

e verifica-se a definição expressa em Eq. 3.2.9. Aplicando-se a regra da cadeia em relação a $Z$, definido como $Z=\frac{\zeta}{L / 2}$ na derivada acima, obtém-se:

$$
-\left.\mathfrak{J}\left\{\frac{2 d \Omega}{L d Z}\right\}\right|_{\eta^{+}}+\left.\mathfrak{J}\left\{\frac{2 d \Omega}{L d Z}\right\}\right|_{\eta^{-}}=\sigma
$$

O parâmetro $\sigma$ é, portanto, definido pela diferença da derivada do potencial complexo $(\Omega)$ entre pontos logo à esquerda e pontos logo à direita do elemento. Assim, a ordem da distribuição de esforços encontrada em $\sigma$ é dada pela ordem dos esforços da descontinuidade do potencial ( $\lambda$ ) menos 1 (um). Por exemplo, no caso de $\sigma$ constante, a distribuição de $\Omega$ ao longo do elemento será 1 (um), o que determina a ordem da linesink.

A distribuição de descargas que descreve a descontinuidade da corrente através de um linesink ordem dois é dada por Strack (1989, pág. 450) pelo seguinte polinômio: 


$$
\sigma=\frac{1}{2}(\stackrel{2}{\sigma}-\stackrel{1}{\sigma}) \Delta+\frac{1}{2}(\stackrel{2}{\sigma}+\stackrel{1}{\sigma})
$$

Observe-se que a distribuição dada contém um termo constante igual à média dos

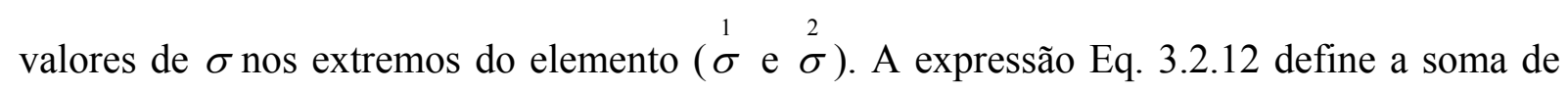
uma distribuição linear com uma distribuição constante. Assim, o elemento linesink de ordem dois é dado pela sobreposição do elemento de ordem um a um potencial complementar encontrado para o termo linear da distribuição descrita na equação Eq. 3.2.12. Substituindo-se a expressão Eq. 3.2.12 em Eq. 2.3.4:

$$
\Omega=\frac{L}{8 \pi} \int_{-1}^{1}\left(\sigma^{2}-\stackrel{1}{\sigma}\right) \Delta \ln (Z-\Delta) d \Delta+\Omega_{1}
$$

onde $\Omega_{1}$ é dado por Eq. 2.3 .5 para $\sigma=\frac{1}{2}(\stackrel{2}{\sigma}+\stackrel{1}{\sigma})$. A integração expressa na Eq. 3.2 .13 fornece:

$$
\stackrel{*}{\Omega}=-\frac{L}{16 \pi}(\stackrel{2}{\sigma}-\stackrel{1}{\sigma})\left[\left(Z^{2}-1\right) \ln \frac{Z-1}{Z+1}+2 Z\right]
$$

E, finalmente, o potencial para uma linesink de ordem dois é escrito como:

$$
\begin{aligned}
& \Omega_{2}=\frac{L}{16 \pi}\left\{-(\stackrel{2}{\sigma}-\stackrel{1}{\sigma})\left[\left(Z^{2}-1\right) \ln \frac{Z-1}{Z+1}+2 Z\right]+\right. \\
& +2\left({ }^{2}+\frac{1}{\sigma}\right)[(Z+1) \ln (Z+1)-(Z-1) \ln (Z-1)+ \\
& \left.\left.+2 \ln \left[\frac{1}{2}\left(2^{2}-1^{\prime}\right)\right]-2\right]\right\}
\end{aligned}
$$

\subsubsection{Lago Poligonal de Ordem Dois}

Um lago poligonal pode ser representado utilizando-se elementos linesink. Dada a distribuição de esforços da Eq. 3.2.12, o balanço hídrico de um lago é obtido integrando-se $\sigma$ sobre os $n$ linesinks da seguinte forma: 


$$
Q=\sum_{i=1}^{n} \frac{\sigma_{1} \sigma_{1}+\sigma_{2}}{2}
$$

Dessa forma, os esforços presentes em cada elemento devem ser calculados levando-se em conta o balanço hídrico no lago. A solução dos parâmetros $(\sigma)$ utilizados no lago é obtida, minimizando-se o potencial no lago (informação verbal de Strack, 2003b) ${ }^{6}$ da seguinte forma:

$$
d \Phi=\sum_{i=1}^{n} \frac{\partial \Phi}{\partial \sigma_{i}} d \sigma_{i}
$$

Observando-se a definição da equação Eq. 3.2.4, o termo $\frac{\partial \Phi}{\partial \sigma_{j}}$ é substituído por cada função de influência $\Lambda_{j}$. Substituindo-se ainda os operadores de derivada total (d) por intervalos discretos $(\Delta)$, tem-se:

$$
\Delta \Phi=\sum_{i=1}^{n} \Lambda_{i} \Delta \sigma_{i}
$$

O operador $\Delta$ refere-se à variação de potencial $\Phi$ devido a $\Delta \sigma_{i}$ em cada $\sigma_{i}$ durante o processo de minimização. Assim, dá-se início ao processo arbitrando-se um potencial $\stackrel{0}{\Phi}=0$ como aproximação inicial do valor mínimo. Como o potencial no lago é dado pela sobreposição de $n$ linesinks, pode-se escrever o potencial devido a um lago na forma da equação Eq. 3.2.4 como $\Phi=\sum \sigma_{i} \Lambda_{i}$ e então $\sum \stackrel{0}{\sigma_{i}} \Lambda_{i}=0$. Por conseguinte, definindo-se $\Delta \Phi=\stackrel{1}{\Phi}-\stackrel{0}{\Phi}$ e $\Delta \sigma=\stackrel{1}{\sigma}-\stackrel{0}{\sigma}:$

$$
\stackrel{1}{\Phi}=\sum_{i=1}^{n} \Lambda_{i} \sigma_{i}
$$

\footnotetext{
${ }^{6}$ Informação fornecida por Otto D.L. Strack durante mini-curso em São Carlos em dezembro de 2003
} 
A variável $\stackrel{1}{\Phi}$ será o potencial minimizado no lago e constitui-se em uma incógnita no sistema. O problema torna-se completamente resolvido, então, incluindo-se a equação do balanço hídrico (Eq. 3.2.16) no sistema. Uma vez que a solução do problema não depende de $\stackrel{0}{\Phi}$, o índice sobrescrito é desnecessário.

\subsubsection{Linedipole e Linedoublet de Ordem Dois}

$\mathrm{Na}$ seção 2.3.2, foi apresentada a formulação de linhas dipolos de primeira ordem. Neste capítulo, essas funções passam a serem designadas como linedipole de primeira ordem. A partir da definição de linedipole (Eq. 2.3.16) uma linedoublet é expressa fazendo $\lambda=i \mu$ na Eq. 2.3.16, obtendo-se:

$$
\Omega=-\frac{\lambda(Z)}{2 \pi i} \ln \frac{Z-1}{Z+1}+i p(Z)
$$

O comportamento da Eq. 3.2.20 é semelhante ao da Eq. 2.3.16, ambas funções apresentam descontinuidades ao longo do elemento. Elementos linedipole e linedoublet são constituídos de infinitos dipolos dispostos ao longo do próprio elemento. Elementos linedipole possui dipolos alinhados com o eixo do próprio elemento enquanto os linedoublet são alinhados transversalmente ao eixo (Figura 3.2.1).

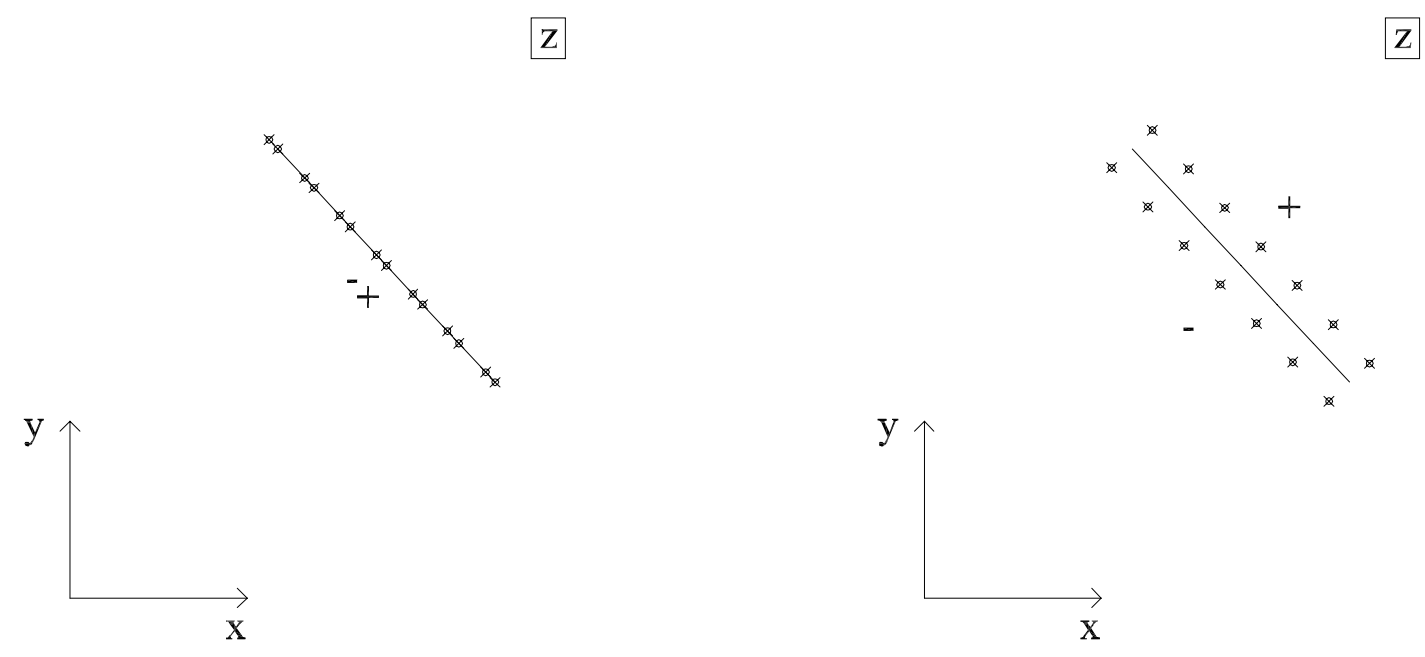

Figura 3.2.1 - Arranjos de dipolos encontrados nos elementos linedipole (à esquerda) e linedoublet (à direita) 
Foi visto que a função linedipole apresenta um intervalo na função de corrente de $\Psi^{+}-\Psi^{-}=\mu(X)$ ao longo do próprio elemento (Eq. 2.3.17), portanto um intervalo na componente imaginária da função $\Omega$. A partir da definição $\arg \left(\frac{Z-1}{Z+1}\right)=\theta$ (Figura 3.2.2) e decompondo-se os termos reais e imaginários da função Eq. 3.2.20 tem-se o seguinte comportamento ao longo do elemento.

$$
\begin{array}{ll}
-1 \leq X \leq 1, \quad Y^{+}=0: & \Omega^{+}=-\frac{\lambda(X)}{2 \pi i}\left[\ln \frac{X-1}{X+1}+i \pi\right]+i p(X) \\
-1 \leq X \leq 1, \quad Y^{-}=0: & \Omega^{+}=-\frac{\lambda(X)}{2 \pi i}\left[\ln \frac{X-1}{X+1}-i \pi\right]+i p(X)
\end{array}
$$

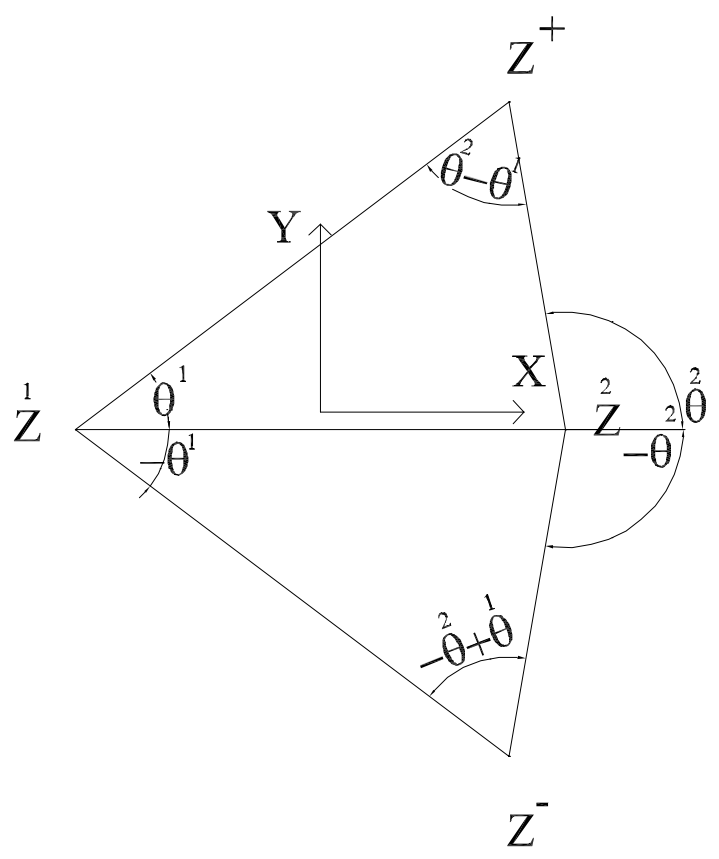

Figura 3.2.2 - Esboço da relação entre distâncias de um ponto ao elemento e seus argumentos

Assim, a Eq. 3.2.20 produz uma descontinuidade no potencial $(\Phi)$ ao longo do elemento $(-1 \leq X \leq 1$ e $Y=0)$ igual ao valor do esforço $\lambda$. Matematicamente, tem-se:

$$
\Phi^{+}-\Phi^{-}=\Re\left(\Omega^{+}-\Omega^{-}\right)=\lambda(X)
$$


O elemento linedoublet de segunda ordem é dado a partir da sobreposição de termos adicionais ao linedoublet de primeira ordem que expressam igualmente termos empregados em elementos linedipole. Strack (1989, pág. 415) define o polinômio $\lambda$ para o elemento de primeira ordem de maneira que $\lambda_{1}$ e $\lambda_{2}$ representem os esforços sobre as extremidades do elemento $j, \delta_{1}$ e $\delta_{2}$, respectivamente. Dessa forma, tem-se:

$$
\lambda(Z)=\lambda_{1} f(Z)+\lambda_{2} g(Z)
$$

onde:

$$
f(Z)=-\frac{1}{2}(Z-1) \text { e } g(Z)=\frac{1}{2}(Z+1)
$$

A definição é agora reescrita como a soma de duas funções $F$ e $G$ de maneira a determinar os coeficientes do polinômio $p(Z)$ na Eq. 3.2.20.

$$
\Omega=\frac{1}{2 \pi i}\left[\lambda_{1} F(Z)+\lambda_{2} G(Z)\right]
$$

Conforme discutido na seção 2.3.2, determina-se $p$ impondo-se que o potencial complexo seja nulo quando $Z \rightarrow \infty$. No caso do linedoublet de primeira ordem, $p$ é reduzido a uma constante e, conforme definido na expressão Eq. 3.2.25, o polinômio $p$ é determinado como - 1 em $F$ e +1 em G. Assim,

$$
\begin{aligned}
& F(Z)=f(Z) \ln \frac{Z-1}{Z+1}-1 \\
& G(Z)=g(Z) \ln \frac{Z-1}{Z+1}+1
\end{aligned}
$$

O linedoublet de ordem dois é obtido, segundo Strack (1989, pág. 452), definindo um termo complementar a ser somado à Eq. 3.2.25 como:

$$
\stackrel{*}{\Omega}=-\frac{L}{2 \pi i}{ }^{*}\left[\left(Z^{2}-1\right) \ln \frac{Z-1}{Z+1}+{ }^{*} p(Z)\right]
$$


Determinado $\stackrel{*}{p}(Z)$ tem-se:

$$
\stackrel{*}{\Omega}=-\frac{L}{2 \pi i}{ }^{*}\left[\left(Z^{2}-1\right) \ln \frac{Z-1}{Z+1}+2 Z\right]
$$

Definindo-se uma variável $\stackrel{c}{\lambda}$ dada pelo valor de $\lambda(Z)$ no centro do elemento escrito da seguinte forma:

$$
\stackrel{c}{\lambda}=\stackrel{*}{\lambda}+\frac{1}{2}\left(\lambda_{1}+\lambda_{2}\right)
$$

pode-se substituir ${ }^{*}$ da Eq. 3.2.30 em Eq. 3.2.29, e definir uma função $F^{*}$ como:

$$
F^{*}(Z)=\left(Z^{2}-1\right) \ln \frac{Z-1}{Z+1}+2 Z
$$

onde o termo polinomial pode ser designado como $f^{*}$ da seguinte forma:

$$
f^{*}(Z)=-\left(Z^{2}-1\right)
$$

Assim, o potencial de descarga é definido como:

$$
\Omega=\frac{1}{2 \pi i}\left\{\lambda_{1}\left[F(Z)+\frac{1}{2} F^{*}(Z)\right]-{ }^{c} F^{*}(Z)+\lambda_{2}\left[G(Z)+\frac{1}{2} F^{*}(Z)\right]\right\}
$$

Strack (1989) examina o comportamento do potencial devido ao elemento linedoublet levando a coordenada complexa $z$ ao limite das extremidades do elemento e obtém as seguintes expressões para pontos próximos aos nós:

$$
\Omega\left(z_{1}\right)=\frac{1}{2 \pi i} \lambda_{2}+\frac{1}{2 \pi i} \lambda_{1} \ln \left(z_{1}-z_{2}\right)
$$




$$
\Omega\left(z_{2}\right)=-\frac{1}{2 \pi i} \lambda_{1}-\frac{1}{2 \pi i} \lambda_{2} \ln \left(z_{2}-z_{1}\right)
$$

As expressões acima evitam que o potencial seja singular nas extremidades do elemento. Embora as expressões evitem as singularidades, não asseguram a suavidade da superfície potenciométrica ao longo dos elementos (Figura 3.2.3, linhas pontilhadas). Neste trabalho, as condições de continuidade e suavidade do escoamento sobre o elemento são asseguradas também sobre as extremidades do elemento por intermédio da distribuição de esforços $\lambda$ (informação verbal de Schulz, 2004) ${ }^{7}$.

Figura 3.2.3 - Potenciometria obtida sem a suavização dos esforços nos extremos dos elementos (linhas pontilhadas) e com a suavização dos esforços (linhas contínuas)

Somando-se as expressões de Eq. 3.2.24 e Eq. 3.2.32 e utilizando-se o termo ${ }^{c}$ definido em Eq. 3.2.30, tem-se uma única expressão que representa o polinômio $\lambda$ em Eq. 3.2.20 da seguinte forma:

$$
\lambda(X)=\left(\frac{\lambda_{2}+\lambda_{1}}{2}-\stackrel{c}{\lambda}\right) X^{2}+\frac{\lambda_{2}-\lambda_{1}}{2} X+\stackrel{c}{\lambda}
$$

\footnotetext{
${ }^{7}$ Informação fornecida por Harry E. Schulz durante discussões cotidianas
} 
Utilizando-se o índice el para a identificação do elemento e o índice $j$ da forma $j=1$, $j+1=c$ e $j+2=2$ a aplicação da Eq. 3.2.36 sobre os nós dos elementos produz:

$$
\stackrel{e l}{\lambda}_{j+2}=\stackrel{e l+1}{\lambda}_{j}
$$

Diferenciando-se Eq. 3.2.36 em relação à coordenada $X$ tem-se:

$$
\frac{d \lambda(X)}{d X}=\left(\lambda_{1}-2 \stackrel{c}{\lambda}+\lambda_{2}\right) X+\frac{\lambda_{2}-\lambda_{1}}{2}
$$

Aplicação da Eq. 3.2.38 sobre os nós dos elementos produz:

$$
\frac{e l}{2} \stackrel{e l}{\lambda}_{j}-2 \stackrel{e l}{\lambda}_{j+1}+\frac{3}{2} \stackrel{e l}{\lambda}_{j+2}=-\frac{3}{2} \stackrel{e l+1}{\lambda}_{j}+2 \stackrel{e l+1}{\lambda}_{j+1}-\frac{1}{2} \stackrel{e l+1}{\lambda}_{j+2}
$$

Na Figura 3.2.3 é ilustrada a melhoria da representação do campo potencial obtida da utilização da expressão nos extremos dos elementos utilizados. Os desenvolvimentos apresentados são válidos da mesma forma para elementos linedipole. O Apêndice A seção (A.2.2), apresenta a utilização de linedipoles para rios e lagos.

\subsubsection{Areasink de Esforço Uniformemente Distribuído}

O potencial devido a uma região finita de recarga é definido em Strack (1989) pela função areasink. A função é definida em todo o espaço plano infinito sobrepondo-se dois problemas: o primeiro regido pela Equação de Poisson no interior da região de recarga e o segundo regido pela Equação de Laplace. Para cada problema define-se um potencial cuja soma produz a expressão desejada:

$$
\Phi=\stackrel{a}{\Phi}+\stackrel{b}{\Phi}
$$

O objetivo da decomposição do problema em duas partes é utilizar as soluções já conhecidas (harmônicas) na resolução do problema. 


$$
\begin{array}{ll}
\nabla^{2} \stackrel{a}{\Phi}=\gamma, & \text { dentro } \\
\nabla^{2} \stackrel{b}{\Phi}=0, & \text { para todo domínio }
\end{array}
$$

O potencial $\stackrel{a}{\Phi}$, que satisfaz a equação no interior da região, é definido como uma solução particular para a Equação de Poisson e no exterior da região $\stackrel{a}{\Phi}=0$. O potencial $\stackrel{b}{\Phi}$, por sua vez, é determinado de maneira a eliminar as descontinuidades ocasionadas por $\stackrel{a}{\Phi}$. Adotando o sobrescrito “+” para a localização de variáveis internas à região e “-” para variáveis localizadas no exterior:

$$
\stackrel{b}{\Phi}^{+}-\stackrel{b}{\Phi}^{-}=\stackrel{b}{\lambda}=-\left(\stackrel{a}{\Phi}^{+}-\stackrel{a-}{\Phi}\right)=-\stackrel{a}{\Phi}^{+}
$$

Observe que ocorre descontinuidade no gradiente de $\stackrel{a}{\Phi}$ através da fronteira: $\frac{\partial \stackrel{a}{\Phi}}{\partial n}=-\stackrel{a}{-}=0$. Assim, $\stackrel{b}{\Phi}$ também deve ser especificada para que a descontinuidade em $Q_{n}$ seja eliminada. Note-se que $\stackrel{b}{\Phi}$ será, então, dado pela soma de duas funções harmônicas cuja sobreposição satisfaz as especificações Eq. 3.2.42 e Eq. 3.2.43, a saber, as funções linedoublet e linesink.

$$
\stackrel{b}{Q}_{n}^{+}-\stackrel{b}{Q}_{n}^{-}=\stackrel{b}{\sigma}=-\left(\stackrel{a}{Q}_{n}^{+}-\stackrel{a}{Q}_{n}^{-}\right)=-\stackrel{a}{Q}_{n}^{+}
$$

Considerando uma região na forma de um polígono qualquer de vértices $z_{j}, j=1,2, \ldots$, n, Strack (1989, pág. 429) sugere que os vértices da poligonal sejam numerados em sentido anti-horário e de modo que o lado entre os vértices $z_{1}$ e $z_{2}$ seja o maior da poligonal (Figura 3.2.4). 

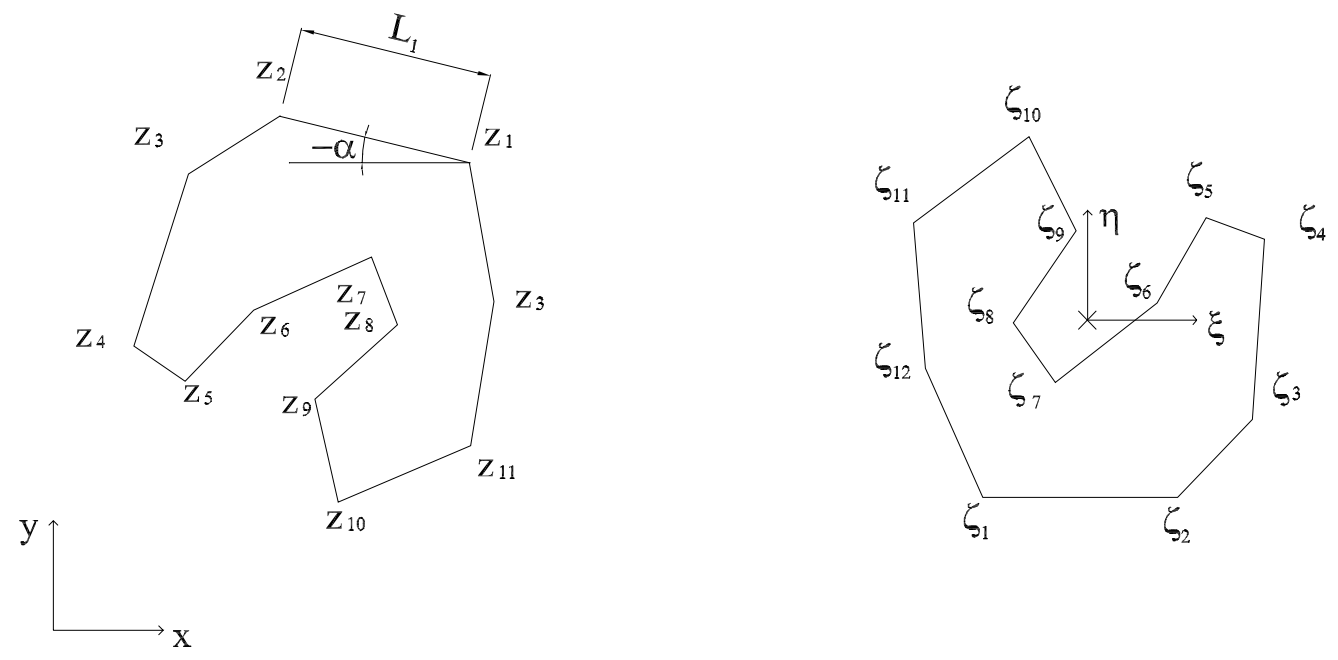

Figura 3.2.4 - Esboço geométrico de uma areasink

As propriedades geométricas da poligonal são definidas da seguinte forma: sejam dois vértices consecutivos ${ }^{b} Z_{j}$ e $Z_{j+1}^{b}$, o comprimento do lado $L_{j}^{b}$ do lado j será dado por:

$$
\stackrel{b}{L}_{j}=\left|\begin{array}{lc}
b & b \\
Z_{j+1}-Z_{j}
\end{array}\right|
$$

como o módulo do número complexo ${ }^{b} Z_{j+1}-Z_{j}$. Escrevendo-se as coordenadas dos vértices agora na forma de vetores ${ }^{b} \mathbf{Z}_{j}$, a área do polígono, por sua vez, será a metade do somatório do produto vetorial:

$$
A=\frac{1}{2} \sum_{j=1}^{n} \mathbf{Z}_{j}^{b} \stackrel{b}{b}_{j+1}
$$

O sistema de coordenadas complexas é, por sua vez, definido tomando-se posições em relação a ${ }^{b} Z_{0}$ e à direção do maior lado por intermédio da seguinte expressão:

$$
\stackrel{b}{\zeta}=\xi+i \eta=\left(z-Z_{0}\right) e^{-i \alpha_{1}}
$$

onde ${ }^{b} Z_{0}$ é definido como o centro geométrico dos vértices da poligonal: 


$$
\mathrm{Z}_{0}=\frac{1}{n} \sum_{j=1}^{n} Z_{j}^{b}
$$

Na Equação de Poisson, a taxa de recarga $\gamma$ é explícita e aparece independente de $\Phi$ sendo portanto, considerada conhecida. Na prática, $\gamma$ independe de. $\Phi$ apenas onde os escoamentos ocorrem em condições livres (não-confinado) e sua distribuição, para efeito de modelagem, é aproximada delimitando-se regiões onde a infiltração pode ser considerada constante. A taxa de recarga, nesses casos, é um parâmetro que está fortemente ligado, dentre outros aspectos, à hidrologia de superfície (precipitação atmosférica e infiltração no solo) que tornam sua distribuição não-uniforme. Dessa forma, $\gamma$ é considerada constante na Eq. 3.2.41 e o potencial que satisfaz essa equação é arbitrado como (Strack, 1989):

$$
\stackrel{a}{\Phi}^{+}=\frac{1}{2} \gamma \xi^{2}
$$

donde se obtém:

$$
\stackrel{a}{Q}_{\xi}^{+}=-\gamma \xi, \stackrel{a}{Q}_{\eta}^{+}=0
$$

De acordo com as equações Eq. 3.2.42 e Eq. 3.2.43:

$$
\stackrel{b}{j}_{j}^{b}=-\stackrel{a}{\Phi}^{+}=-\frac{1}{2} \gamma \xi^{2}
$$

e

$$
\underset{j}{\sigma} \underset{j}{b} \stackrel{a}{Q_{n}^{+}}=\gamma \xi \operatorname{sen}\left(\alpha_{j}-\alpha_{1}\right)
$$

Determinou-se até aqui a distribuição da recarga $(\gamma)$ e as descontinuidades no potencial $\stackrel{a}{\Phi}$ a serem eliminadas pelo potencial $\stackrel{b}{\Phi}$. Obtidos $\underset{j}{\stackrel{b}{\lambda}}$ e $\underset{j}{\sigma}$, a aplicação das funções sobre as 
bordas da poligonal é feita mediante a definição de um sistema de coordenadas adimensional $\mathrm{Z}$ para cada lado $\mathrm{j}$ da poligonal.

$$
Z_{j}=X_{j}+i Y_{j}=\frac{Z-\frac{1}{2}\left(z_{j+1}+z_{j}\right)}{\frac{1}{2}\left(z_{j+1}-z_{j}\right)}=\frac{\zeta-\frac{1}{2}\left(\zeta_{j+1}+\zeta_{j}\right)}{\frac{1}{2}\left(\zeta_{j+1}-\zeta_{j}\right)}
$$

As distribuições dadas pelas equações Eq. 3.2.50 e Eq. 3.2.51 são expressas em cada função de borda em termos de suas próprias coordenadas $Z_{j}$ que expressa $\xi$, isolando $\zeta$ da expressão anterior:

$$
\zeta=\frac{1}{2}\left(\zeta_{j+1}-\zeta_{j}\right) Z_{j}+\frac{1}{2}\left(\zeta_{j+1}+\zeta_{j}\right)
$$

A expressão contém $\xi$ enquanto componente de $\zeta$. Sabendo que as distribuições de esforços dadas nas expressões Eq. 3.2.50 e Eq. 3.2.51 são válidas apenas ao longo de cada lado da poligonal, a componente $\eta$ de $\zeta$ é nula e, portanto, $\zeta$ é reescrita em termos de $\xi$ como segue:

$$
\xi=\frac{1}{2}\left(\xi_{j+1}-\xi_{j}\right) Z_{j}+\frac{1}{2}\left(\xi_{j+1}+\xi_{j}\right)
$$

Assim, as distribuições Eq. 3.2.50 e Eq. 3.2.51 são reescritas como:

$$
\begin{aligned}
& \underset{j}{\lambda}=-\frac{1}{8} \gamma\left(\xi_{j+1}-\xi_{j}\right)\left[\left(\xi_{j+1}-\xi_{j}\right)\left(Z_{j}{ }^{2}-1\right)+2\left(\xi_{j+1}+\xi_{j}\right)\left(Z_{j}+1\right)\right]-\frac{1}{2} \gamma \xi_{j}{ }^{2} \\
& \underset{j}{\sigma}=\gamma\left[\frac{1}{2}\left(\xi_{j+1}-\xi_{j}\right) Z_{j}+\frac{1}{2}\left(\xi_{j+1}+\xi_{j}\right)\right] \operatorname{sen}\left(\alpha_{j}-\alpha_{1}\right)
\end{aligned}
$$

As distribuições $\lambda_{j}^{b}$ e ${ }_{j}^{b}$ podem ser agora utilizadas nas expressões que definem os potenciais cuja sobreposição expressará $\stackrel{b}{\Phi}$. A forma geral a partir das funções linedoublet e 
linesink que definem o potencial $\stackrel{b}{\Phi}$ é dada na equação Eq. 3.2.57 na forma complexa. O procedimento para a obtenção da forma geral do areasink é descrito na seção A.2.3 do Apêndice A.

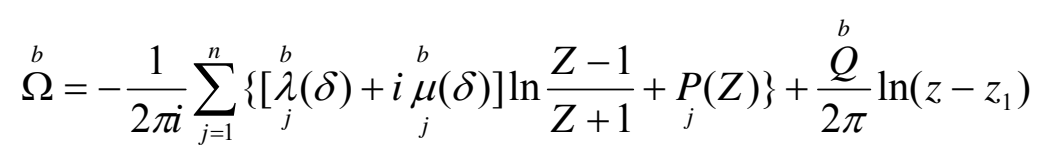

O parâmetro $\stackrel{b}{\mu}$ em Eq. 3.2 .57 pode ser obtido a partir de $\stackrel{b}{\sigma}$ utilizando-se (ver Eq. A.10)

$$
\underset{j}{\sigma}=-\frac{2}{L_{j}} \frac{d}{d Z_{j}} \underset{j}{\mu}
$$

donde $\stackrel{b}{\mu}$ é obtido como:

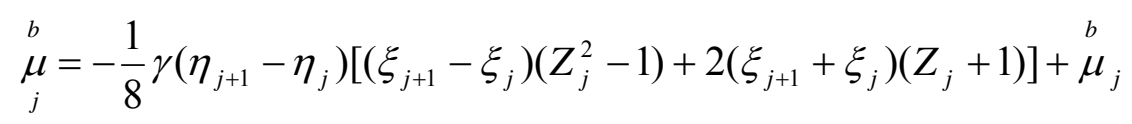

onde $\mu_{j}^{b}=\left[\begin{array}{c}b \\ \mu\end{array}\right]_{\zeta=\zeta_{j}}$ define o valor da função no nó $\zeta_{j}$.

A equação Eq. 3.2.59 define uma expressão recursiva para $\mu_{j}$ onde $\mu_{j+1}^{b}$ é igual a $\underset{j}{\mu}$ no nó $\zeta_{j+1}$, onde $Z_{j}=1$, e adota-se $\mu_{1}^{b}=0$. Assim, a função complexa $\underset{j}{\lambda}+i \underset{j}{b}$ é obtida da soma da equação Eq. 3.2.55 com Eq. 3.2.59 como:

$$
\begin{aligned}
& \underset{j}{\lambda}+i \underset{j}{\stackrel{b}{\mu}=}=-\frac{1}{2} \gamma \xi_{j}^{2}-\frac{1}{8} \gamma\left(\zeta_{j+1}-\zeta_{j}\right)\left[\left(\xi_{j+1}-\xi_{j}\right)\left(Z_{j}^{2}-1\right)+2\left(\xi_{j+1}+\xi_{j}\right)\left(Z_{j}+1\right)\right]+i \underset{j}{\mu} \\
& \stackrel{b}{\Omega}=\sum_{j=0}^{n} \Omega_{j}^{b}+\frac{\stackrel{Q}{2 \pi}}{2 \pi} \ln \left(z-Z_{1}\right)
\end{aligned}
$$


onde $\stackrel{b}{Q}=\gamma A$ é o total descarregado da ou para a poligonal.

Procedendo-se conforme apresentado na seção A.2.3 do Apêndice A, é obtido o termo de correção do escoamento regional $\left(P_{j}\right)$ em Eq. 3.2.57 e o elemento areasink para $\gamma$ constante é finalmente expresso por:

$$
\stackrel{b}{\Phi}=\mathfrak{R}\left\{\frac{\gamma}{2 \pi i} \sum_{j=1}^{n}\left(c_{j}^{2} \zeta^{2}+\underset{j}{c} \zeta+\underset{j}{c}\right) \ln \frac{\zeta-\zeta_{j+1}}{\zeta-\zeta_{j-1}}+\frac{\gamma A}{2 \pi} \ln \left(z-z_{1}\right)-\frac{3 \gamma A}{4 \pi}\right\}
$$

Como definido na equação Eq. 3.2.48, $\stackrel{a}{\Phi}$ deve ser adicionada à parte real de $\stackrel{b}{\Phi}$ no interior do polígono. Strack (1989) define a seguinte função complexa, cuja parte real vale $\frac{1}{2} \gamma \xi^{2}$ no interior do polígono e desaparece no exterior:

$$
\stackrel{a}{\Phi}=\frac{\gamma}{4 \pi i} \xi^{2} \sum_{j=1}^{n} \ln \frac{\zeta-\zeta_{j+1}}{\zeta-\zeta_{j}}
$$

\subsection{Elementos Transientes}

Os elementos apresentados nestas seção seguem a formulação de Zaadnoordijk (1988). Essa formulação consiste de três passos: (1) definição de uma solução fundamental da Equação do Calor (Eq. 3.3.1) adequada ao elemento desejado; (2) um polinômio para a distribuição de esforços no elemento; e (3) um polinômio para a evolução dos coeficientes da distribuição de esforços no elemento. Convenciona-se chamar ordem do elemento o maior expoente do polinômio sobre o eixo do elemento. Convenciona-se, ainda, chamar grau o maior expoente da evolução dos coeficientes do polinômio do elemento no tempo.

$$
\left[\frac{\partial^{2} \Phi}{\partial x^{2}}+\frac{\partial^{2} \Phi}{\partial y^{2}}\right]+N=\frac{1}{\alpha} \frac{\partial \Phi}{\partial t}
$$

Para aqüíferos confinados, o coeficiente de difusão $(\alpha)$ é dado por $\alpha=\frac{K H}{S}$. Para aqüíferos livres, a Equação de Boussinesq é linearizada utilizando-se a definição da Eq. 3.1.5a 
para o potencial e a introdução de uma espessura média do aqǘfero $\bar{\phi}$ (Strack 1989, pág. 195). O coeficiente de difusão, neste caso, é dado por $\alpha=\frac{K \bar{\phi}}{n_{e}}$. A condição inicial das soluções é dada pela solução trivial. Assim, o potencial que expressa a condição inicial de um modelo realista pode ser dado por uma solução do modelo para um tempo qualquer ou mesmo para o regime estacionário. A condição final das soluções da Eq. 3.3.1 utilizadas como elementos é especificada pela estacionaridade da função em $t \rightarrow \infty$.

Dessa forma, o potencial $\Phi(\mathrm{t})$ é dado com base na sobreposição histórica de efeitos e expresso da seguinte forma:

$$
\Phi\left(t_{k}\right)=\sum_{m=0}^{k} \Phi_{m}, \quad \text { para } k>0
$$

Assim, para $t=0$, tem-se:

$$
\Phi_{k}=\Phi\left(t_{k}\right), \quad \text { para } k=0
$$

São apresentadas a seguir soluções de problemas elementares de potenciais devidos a: um lago circular, um linesink ordem um e grau zero, onde, neste trabalho é feita a extensão para ordem dois; um lago poligonal; um linedoublet (também estendido para ordem dois grau zero neste trabalho) e uma areasink poligonal. Em formulações mais recentes, o problema da sobreposição de efeitos em escoamentos transientes tem sido abordado também a partir da Equação de Helmholtz no domínio dado pela transformada de Laplace. A técnica consiste em sobrepor soluções obtidas para a Equação de Helmholtz e transportá-las para o domínio real via transformada de Laplace inversa (Furman e Neuman 2003, 2004).

\subsubsection{Potencial de Descarga Devido a um Lago Circular}

O problema do lago circular é analisado de forma idêntica à apresentada na seção 3.2.1. O lago é sujeito a um escoamento regional e a variações de sua condição de equilíbrio estacionário. A sobreposição de um dipolo com orientação e intensidade específicas sobre o escoamento regional produz uma equipotencial circular coincidente com a borda do lago. Por sua vez, as tensões hidrológicas sobre a superfície do lago são representadas por uma vazão 
totalizada em um poço concêntrico ao lago. A função utilizada para a representação de transitórios em um poço é dada pela solução de Theis escrita em Strack (1989, pág. 196) como:

$$
\underset{p 0_{k}}{\Phi}=-\frac{Q}{4 \pi} \mathrm{E} 1\left(w^{2}\right)+\Phi_{0}
$$

onde $E 1$ é a integral exponencial $\int_{w^{2}}^{\infty} \frac{\exp (-v)}{v} d v$, também conhecida como função poço e $w^{2}$ é o adimensional $\frac{r^{2}}{4 \alpha\left(t-t_{k}\right)}$. Diversas aproximações são encontradas para a função E1 (e.g. Strack, 1989, pág. 198).

A função dipolo a ser utilizada na obtenção da equipotencial desejada para a borda do lago é obtida a partir da expressão dada por Carslaw e Jaeger (1959 apud Zaadnoordijk (1988) para o dipolo instantâneo da seguinte forma:

$$
\Phi=\frac{1}{8 \pi \alpha\left(t-t_{0}\right)^{2}}\left[s_{x} r \cos \theta+s_{y} r \operatorname{sen} \theta\right] \exp \left(-\frac{r^{2}}{4 \alpha\left(t-t_{0}\right)}\right)
$$

O dipolo responsável pela geração da equipotencial do lago sujeito a um esforço constante ao longo do tempo será encontrado fazendo $\underset{d 0 k}{\Phi}=\int_{0}^{t} \underset{d i}{\Phi d} \tau$, donde obtém-se:

$$
\underset{d 0 k}{\Phi}=\frac{\left[s_{x} r \cos \theta+s_{y} r \operatorname{sen} \theta\right]}{8 \pi \alpha} \int_{0}^{t} \frac{1}{(t-\tau)^{2}} \exp \left(-\frac{r^{2}}{4 \alpha(t-\tau)}\right) d \tau
$$

Efetuando-se agora a seguinte mudança de variáveis conforme a definição de $w$ na Eq. 3.3.4:

$$
w=\sqrt{\frac{r^{2}}{4 \alpha(t-\tau)}}, t-\tau=\frac{r^{2}}{4 \alpha w^{2}}, d \tau=\frac{r^{2}}{4 \alpha w^{3}} d w
$$

obtém-se: 


$$
\underset{d 0 k}{\Phi}=\frac{\left[s_{x} r \cos \theta+s_{y} r \operatorname{sen} \theta\right]}{2 \pi r^{2}} \int_{\sqrt{\frac{r^{2}}{4 \alpha t}}}^{\infty} w \exp \left(-w^{2}\right) d w
$$

cuja solução é dada por:

$$
\Phi_{d 0 k}=\frac{\left[s_{x} r \cos \theta+s_{y} r \operatorname{sen} \theta\right]}{4 \pi r^{2}} \exp \left(-\frac{r^{2}}{4 \alpha\left(t-t_{k}\right)}\right)
$$

Da forma semelhante à que ocorre com as funções harmônicas, as funções obtidas da Equação do Calor podem ser referidas como He e escritas na forma:

$$
\underset{k}{\Phi}=H e=\lambda_{(j)}^{k} \Lambda_{(j)}^{k}
$$

Cada termo das funções $H e$, porém, é definido com base na sobreposição de efeitos ao longo do tempo (Zaadnoordijk, 1988 e Zaadnoordijk e Strack, 1993). Tomando como exemplo a função Eq. 3.3.4, tem-se:

$$
\stackrel{k}{\Lambda}_{1}=-\frac{1}{4 \pi} \operatorname{E} 1(w) \quad(k>0)
$$

$$
\stackrel{k}{\Lambda}_{1}=0 \quad(k=0)
$$

Fazendo ${ }^{k} \lambda_{1}=\stackrel{k}{Q}$ ou ainda ${ }^{k}{ }_{j}=s_{j}^{k}(j=x, y)$ em Eq. 3.3.9, conforme as equações Eq. 3.3.3 e Eq. 3.3.2, tem-se os parâmetros definidos no instante $k$ como:

$$
\stackrel{k}{\lambda}_{j}=\lambda_{j}\left(t_{k}\right)-\sum_{m=0}^{k-1} \lambda_{j}^{m}, \quad \text { para } k>0
$$

$$
\stackrel{k}{k}_{j}=0, \quad \text { para } k=0
$$


onde o tempo inicial é identificado como $t_{k=0}$. Assim, tanto a função de influência $(\Lambda)$ quanto o parâmetro de esforço $(\lambda)$ terão valores diferentes de zero somente para instantes de tempo superiores a $t_{k}=0$.

\subsubsection{Linesink de Ordem Dois e Grau Zero}

O elemento linesink é definido segundo Zaadnoordijk (1988) como o efeito obtido da sobreposição de infinitos poços ao longo de uma linha finita. A partir da função Eq. 3.3.4, Zaadnoordijk (1988) obtém expressões que definem elementos de primeira ordem e grau arbitrário, ou seja, esforços uniformemente distribuídos no elemento e evolução temporal de grau tão alto quanto se queira. Embora o elemento obtido por Zaadnoordijk (1988) seja de grau arbitrário (relativamente à distribuição temporal de esforços), a distribuição espacial por ele descrita (primeira ordem), não favorece a sobreposição das extremidades dos elementos. As condições para a utilização de linesinks encadeadas são discutidas nesta seção. Para isso, é obtida uma solução que expressa o elemento linesink com distribuição linear e constante no tempo (ordem dois e grau zero) e são discutidas as condições que devem ser atendidas pelo elemento quando utilizado em cadeia.

A notação segue a convenção definida por Zaadnoordijk (1988). A numeração no sobrescrito de $\Phi$ refere-se à ordem do polinômio utilizado na distribuição de esforços sobre o elemento. As letras utilizadas nos subscritos estão designadas como: ls referindo-se a linesink, $i$ ou o número 0 (zero) referindo-se respectivamente a instantâneo e grau zero e $l$ ou $q$ referindo-se a linear e quadrático, respectivamente. Matematicamente, os elementos linesink escritos em Zaadnoordijk (1988) são obtidos por integração da solução fundamental de Carslaw e Jaeger (1959) para um pulso pontual instantâneo no plano horizontal:

$$
\underset{p s i}{\Phi}=-\frac{\sigma}{4 \pi\left(t-t_{0}\right)} \exp \left(-\frac{(\xi-\stackrel{0}{\xi})^{2}+(\eta-\stackrel{0}{\eta})^{2}}{4 \alpha\left(t-t_{0}\right)}\right)
$$

onde os subscritos estão designados da seguinte forma: ps para pointsink e $i$ para instantâneo.

A integração da solução fundamental é feita definindo-se uma densidade de esforços constante sobre o elemento (Zaadnoordijk, 1988 Eq. 4.38). O procedimento para a construção da integral que representa a sobreposição dos pointsinks constituintes de uma linesink é apresentado na seção 2.3.1. 


$$
\underset{l s i}{\Phi}=\int_{\substack{\xi \\ \xi}}^{\xi_{c}}-\sigma \frac{1}{4 \pi\left(t-t_{0}\right)} \exp \left(-\frac{(\xi-u)^{2}+\eta^{2}}{4 \alpha\left(t-t_{0}\right)}\right) d u
$$

O potencial devido a uma linesink de ordem um (uniforme ao longo do elemento, ver Eq. 3.2.11) com densidade de esforços constante no tempo é dado por Zaadnoordijk (1988, Eq. 4.56) pela integral de $\underset{\text { lsi }}{\Phi}$ no tempo da seguinte forma:

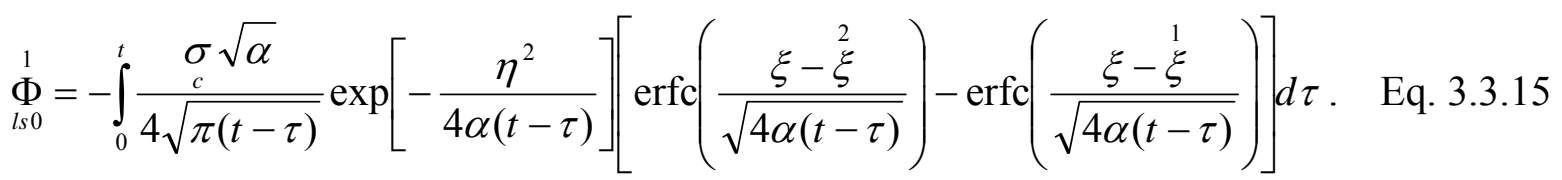

Efetuando-se a mudança de variáveis como apresentado na Eq. 3.3.7:

$$
w=\sqrt{\frac{\eta^{2}}{4 \alpha(t-\tau)}}, t-\tau=\frac{\eta^{2}}{4 \alpha w^{2}}, d \tau=\frac{\eta^{2}}{4 \alpha w^{3}} d w
$$

a solução da integral, por sua vez, é expressa por:

$$
\underset{l s 0}{\Phi}=-\underset{0}{\sigma} \frac{\xi|\eta|}{4 \sqrt{\pi}} I_{1}^{0}
$$

onde $\stackrel{0}{I_{1}}$ é dada em Zaadnoordijk (1988, Eq. 4.43). Barnes e Strack (2003) apresentam correções a se fazer em alguns passos algébricos adotados no trabalho de Zaadnoordijk (1988) devidos à mudança de variáveis (Eq. 3.3.16). Assim, a função $\stackrel{0}{I_{1}}$ corrigida é: 


$$
\begin{aligned}
& \stackrel{0}{I_{1}}=\sqrt{\frac{4 \alpha t}{\eta^{2}}} e^{-\frac{\eta^{2}}{4 \alpha t}}\left[\operatorname{erfc}\left(\frac{\xi-\stackrel{2}{\xi}}{|\eta|} \sqrt{\frac{\eta^{2}}{4 \alpha t}}\right)-\operatorname{erfc}\left(\frac{\xi-\xi}{|\eta|} \sqrt{\frac{\eta^{2}}{4 \alpha t}}\right)\right]+ \\
& -\frac{1}{\sqrt{\pi}}\left[\frac{\xi-\stackrel{2}{\xi}}{|\eta|} \mathrm{E} 1\left(\frac{\left(\xi-\stackrel{2}{\xi}^{2}+\eta^{2}\right.}{4 \alpha t}\right)-\frac{\xi-\xi^{\prime}}{|\eta|} \mathrm{E} 1\left(\frac{\left(\xi-\xi^{2}\right)^{2}+\eta^{2}}{4 \alpha t}\right)\right]+ \\
& -2 \int_{\sqrt{\frac{\eta^{2}}{4 \alpha t}}}^{\infty} \exp \left(-w^{2}\right)\left[\operatorname{erfc}\left(w \frac{\xi-\xi^{2}}{|\eta|}\right)-\operatorname{erfc}\left(w \frac{\xi-\xi}{|\eta|}\right)\right] d w
\end{aligned}
$$

Dessa forma, o elemento linesink de primeira ordem é expresso por:

$$
\begin{aligned}
& \stackrel{1}{\Phi}=-\frac{\sigma}{4} \sqrt{\frac{4 \alpha t}{\pi}} \exp \left(-\frac{\eta^{2}}{4 \alpha t}\right)\left[\operatorname{erfc}\left(\frac{\xi-\xi}{\sqrt{4 \alpha t}}\right)-\operatorname{erfc}\left(\frac{\xi-\xi}{\sqrt{4 \alpha t}}\right)\right]+
\end{aligned}
$$

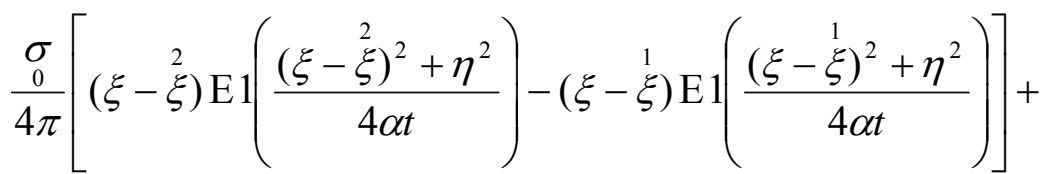

$$
\begin{aligned}
& \frac{\sigma 0}{2 \sqrt{\pi}} \int_{\sqrt{\frac{\eta^{2}}{4 \alpha t}}}^{\infty} \exp \left(-w^{2}\right)\left[\operatorname{erfc}\left(w \frac{\xi-\stackrel{2}{\xi}}{|\eta|}\right)-\operatorname{erfc}\left(w \frac{\xi-\stackrel{1}{\xi}}{|\eta|}\right)\right] d w
\end{aligned}
$$

A integral no último termo da expressão Eq. 3.3.19 é referida neste trabalho como Integral de Rosser:

$$
\int_{\sqrt{\frac{\eta^{2}}{4 \alpha t}}}^{\infty} \exp \left(-w^{2}\right)\left[\operatorname{erfc}\left(w \frac{\xi-\xi^{2}}{|\eta|}\right)-\operatorname{erfc}\left(w \frac{\xi-\xi}{|\eta|}\right)\right] d w
$$


devido ao pioneirismo de Rosser (1948 apud Zaadnoordijk, 1988) ${ }^{8}$ no problema. Litkouhi e Becker (1982 apud Zaadnoordijk, 1988) ${ }^{9}$ utilizaram a integral em um problema de condução de calor e muito contribuíram para a análise do problema.

Embora o elemento linesink de ordem um mantenha o esforço invariável ao longo de seu comprimento, os esforços $(\underset{c}{\sigma})$ dos elementos podem ser especificados individualmente constituindo-se segmentos de polilinhas (e.g. especificações de condições de contorno sobre rios e riachos). Entretanto, sua aplicação em casos de condições não constantes introduz efeitos indesejados nas extremidades dos elementos. A aplicação de elementos linesink (e.g., especificação de contornos) ao longo de polilinhas requer que a distribuição dos esforços seja contínua. Considere-se para tanto que a distribuição seja linear, de maneira que os valores dos esforços coincidam nas extremidades de cada elemento. A distribuição de esforços ao longo do elemento linesink poderá ser definida como

$$
\sigma(\xi)=\underset{c}{\sigma}+\underset{l}{\sigma} \xi
$$

onde o subscrito $l$ refere-se à palavra linear.

Em uma cadeia de elementos, a continuidade entre os valores de esforços em cada nó $i$ será assegurada fazendo:

$$
\sigma^{i}\left(-\frac{i}{2}\right)=\sigma\left(\frac{i-1}{L}\right)=\sigma_{c}^{i}-\sigma_{l}^{i} \frac{i}{L}=\sigma_{c}+\underset{l}{\sigma} \frac{i-1}{2} \frac{i-1}{2}
$$

onde $\stackrel{i}{L}$ é o comprimento do elemento $i$.

Neste trabalho observa-se que a distribuição de potencial ao longo do elemento, $\Phi(\xi, 0)$, não depende da distribuição de esforços $\sigma$. Sabendo que $\sigma$ é definido apenas em função da

8 Rosser, J.B. (1948) Theory and Application of $\int_{0}^{z} \exp \left(-x^{2}\right) d x$ and $\int_{0}^{z} \exp \left(-p^{2} y^{2}\right) d y \int_{0}^{y} \exp \left(-x^{2}\right) d x$, Mapleton House, Brooklyn

${ }^{9}$ Litkouhi e Becker (1982) Temperatures in Semi-Infinite Body Heated by Constant Heat Flux Over Half Space. In: Proceedings of the Seventh International Heat Transfer Conference Muenchen, v2, pp21-27, Washington DC 
direção transversal do elemento (Eq. 3.2.9) e aplicando-se a definição de linhas de corrente $\Psi$ Eq. 2.1.14, tem-se:

$$
\sigma=\frac{\partial\left(\Psi^{+}-\Psi^{-}\right)}{\partial \xi}=-Q_{\eta}^{+}-Q_{\eta}^{-}=-2 Q_{\eta}
$$

A partir da definição de potencial de descarga no plano(Eq. 2.1.5), por sua vez, tem-se:

$$
Q_{\xi}=-\frac{\partial \Phi}{\partial \xi}, Q_{\eta}=-\frac{\partial \Phi}{\partial \eta}
$$

Integrando-se o potencial na direção do eixo do elemento, tem-se uma função apenas da direção $\xi$ da seguinte forma:

$$
\Phi(\xi, 0)=-\int_{0}^{\xi} Q_{\xi} d \xi
$$

Assim, conclui-se que para se ter a continuidade de $\sigma$ assegurada, é necessário incluir a expressão Eq. 3.3.22 na formulação do elemento e, portanto, obter-se a expressão de segunda ordem do elemento.

Neste trabalho, o potencial devido a um linesink de segunda ordem é obtido escrevendose o polinômio de esforços conforme a Eq. 3.3.21. Observa-se que a expressão do elemento poderá ser obtida como a soma do potencial de ordem um com o potencial obtido de uma distribuição linear $(\underset{l}{\sigma} \xi)$

$$
\stackrel{2}{\Phi}=-\int_{\substack{1 \\ \xi}}^{\xi_{c}^{\xi}}(\underset{c}{\sigma}+\underset{l}{\sigma} u) \frac{1}{4 \pi\left(t-t_{0}\right)} \exp \left(-\frac{(\xi-u)^{2}+\eta^{2}}{4 \alpha\left(t-t_{0}\right)}\right) d u
$$

Observe-se que operando-se a integração do primeiro termo, obtém-se a expressão do potencial $\underset{\text { lsi }}{\stackrel{1}{\Phi}}$ (Eq. 3.3.14). Assim, é necessário apenas obter-se a integração do segundo termo da equação Eq. 3.3.26: 


$$
\underset{\text { lsil }}{\Phi}=-\int_{\substack{1 \\ \xi}}^{\xi_{l}^{\xi}} \sigma \frac{u}{4 \pi\left(t-t_{0}\right)} \exp \left(-\frac{(\xi-u)^{2}+\eta^{2}}{4 \alpha\left(t-t_{0}\right)}\right) d u
$$

onde $\underset{\text { lsil }}{\Phi}$ é o potencial devido à distribuição complementar $\underset{l}{\sigma} \xi$.

O potencial devido a uma linesink com densidade de esforços linear (Eq. 3.3.21) mantida constante no tempo $(\stackrel{2}{\Phi})$ poderá ser obtido integrando-se agora $\underset{l s i}{\Phi}$ em relação ao tempo. Escrevendo-se $\underset{\text { ls0 }}{\stackrel{2}{9}}$ a partir da definição expressa em Eq. 3.3.26, tem-se:

$$
\stackrel{2}{\Phi}=\int_{0}^{t} \underset{l s i}{\Phi} d \tau=\int_{0}^{t} \underset{l s i}{\Phi}+\underset{\text { lsil }}{\Phi} d \tau
$$

O potencial de densidade constante Eq. 3.3.19, como é definido por Zaadnoordijk (1988, Eq. 4.40) é obtido do primeiro termo da integral na equação Eq. 3.3.28, portanto, $\underset{\text { Is } 0}{\stackrel{2}{9}}$ poderá ser obtido novamente integrando-se apenas o potencial complementar $(\underset{\text { lsil }}{\Phi})$ da seguinte forma:

$$
\begin{aligned}
& \int_{0}^{t} \Phi=-\frac{l^{\prime}}{4} \int_{0}^{t} \exp \left(-\frac{\eta^{2}}{4 \alpha(t-\tau)}\right) \times \\
& \left\{\xi \frac{\sqrt{\alpha}}{\sqrt{\pi(t-\tau)}}\left[\operatorname{erfc}\left(\frac{\xi-\xi^{2}}{\sqrt{4 \alpha \pi(t-\tau)}}\right)-\operatorname{erfc}\left(\frac{\xi-\xi}{\sqrt{4 \alpha \pi(t-\tau)}}\right)\right]+\right. \\
& \left.\frac{2 \alpha}{\pi}\left[\exp \left(-\frac{(\xi-\xi)^{2}}{4 \alpha(t-\tau)}\right)-\exp \left(-\frac{\left(\xi-\xi^{2}\right)^{2}}{4 \alpha(t-\tau)}\right)\right]\right\} d \tau
\end{aligned}
$$

Efetuando-se a mudança de variáveis como apresentado na Eq. 3.3.7:

$$
w=\sqrt{\frac{\eta^{2}}{4 \alpha(t-\tau)}}, t-\tau=\frac{\eta^{2}}{4 \alpha w^{2}}, d \tau=\frac{\eta^{2}}{4 \alpha w^{3}} d w
$$


produz-se:

$$
\int_{0}^{t} \underset{\text { lsil }}{\Phi} d \tau=-\sigma \frac{\xi|\eta|}{4 \sqrt{\pi}} I_{1}^{0}-\sigma \frac{\eta^{2}}{4 \pi} I_{2}^{1}
$$

onde $\stackrel{0}{I_{1}}$ é dada na Eq. 3.3.18 e $\stackrel{1}{I_{2}}$, após as correções propostas por Barnes e Strack (2003) para Zaadnoordijk (1988, Eq. 4.46), deve ser escrita como:

$$
\begin{aligned}
& I_{2}=\frac{1}{2} \frac{4 \alpha t}{\eta^{2}}\left[\exp \left(-\frac{(\xi-\xi)^{2}+\eta^{2}}{4 \alpha t}\right)-\exp \left(-\frac{\left(\xi-\xi^{2}\right)^{2}+\eta^{2}}{4 \alpha t}\right)\right]+ \\
& \frac{1}{2}\left[\frac{\left(\xi-\xi^{2}\right)^{2}+\eta^{2}}{\eta^{2}} \mathrm{E} 1\left(\frac{\left(\xi-\xi^{2}\right)^{2}+\eta^{2}}{4 \alpha t}\right)-\frac{\left(\xi-\xi^{2}\right)^{2}+\eta^{2}}{\eta^{2}} \mathrm{E} 1\left(\frac{(\xi-\xi)^{2}+\eta^{2}}{4 \alpha t}\right)\right]
\end{aligned}
$$

Por fim, a integral $\int_{0}^{t} \underset{\text { lsil }}{\Phi d} \tau$ produz:

$$
\begin{aligned}
& \int_{0}^{t} \Phi d \tau=-\frac{\sigma \xi}{4 \sqrt{\pi}}\left\{\sqrt{4 \alpha t} \exp \left(-\frac{\eta^{2}}{4 \alpha t}\right)\left[\operatorname{erfc}\left(\frac{\xi-\xi}{\sqrt{4 \alpha t}}\right)-\operatorname{erfc}\left(\frac{\xi-\xi}{\sqrt{4 \alpha t}}\right)\right]+\right. \\
& \frac{1}{\sqrt{\pi}}\left[\frac{\xi-\stackrel{2}{\xi}}{|\eta|} \mathrm{E} 1\left(\frac{(\xi-\stackrel{2}{\xi})^{2}+\eta^{2}}{4 \alpha t}\right)-\frac{\xi-\stackrel{1}{\xi}}{|\eta|} \mathrm{E} 1\left(\frac{\left(\xi-\stackrel{1}{\xi}^{2}+\eta^{2}\right.}{4 \alpha t}\right)\right]+ \\
& \left.2 \int_{\sqrt{\frac{\eta^{2}}{4 \alpha t}}}^{\infty} \exp \left(-w^{2}\right)\left[\operatorname{erfc}\left(w \frac{\xi-\stackrel{2}{\xi}^{2}}{|\eta|}\right)-\operatorname{erfc}\left(w \frac{\xi-\xi}{|\eta|}\right)\right] d w\right\}+ \\
& -\sigma \frac{1}{2} \frac{\eta^{2}}{4 \pi}\left\{\frac{\left(\xi-\stackrel{2}{\xi}^{2}+\eta^{2}\right.}{\eta^{2}} \mathrm{E} 1\left(\frac{\left(\xi-\stackrel{2}{\xi}^{2}\right)^{2}+\eta^{2}}{4 \alpha t}\right)-\frac{\left(\xi-\mathfrak{1}^{2}\right)^{2}+\eta^{2}}{\eta^{2}} \mathrm{E} 1\left(\frac{(\xi-\xi)^{2}+\eta^{2}}{4 \alpha t}\right)+\right. \\
& \left.\frac{1}{2} \frac{4 \alpha t}{\eta^{2}}\left[\exp \left(-\frac{(\xi-\stackrel{1}{\xi})^{2}+\eta^{2}}{4 \alpha t}\right)-\exp \left(-\frac{\left(\xi-\stackrel{2}{\xi}^{2}+\eta^{2}\right.}{4 \alpha t}\right)\right]\right\}
\end{aligned}
$$


A expressão para o elemento de linesink ordem dois e grau zero $(\stackrel{2}{\Phi})$ poderá, então, ser escrita a partir das equações Eq. 3.3.28 e Eq. 3.3.33 como:

$$
\begin{aligned}
& \stackrel{2}{\Phi}=-\left(\sigma_{l} \xi \sqrt{\frac{\alpha t}{4 \pi}}+\sigma \sqrt{\frac{\alpha t}{4 \pi}}\right) \exp \left(-\frac{\eta^{2}}{4 \alpha t}\right)\left[\operatorname{erfc}\left(\frac{\xi-\stackrel{2}{\xi}}{\sqrt{4 \alpha t}}\right)-\operatorname{erfc}\left(\frac{\xi-\xi}{\sqrt{4 \alpha t}}\right)\right]+
\end{aligned}
$$

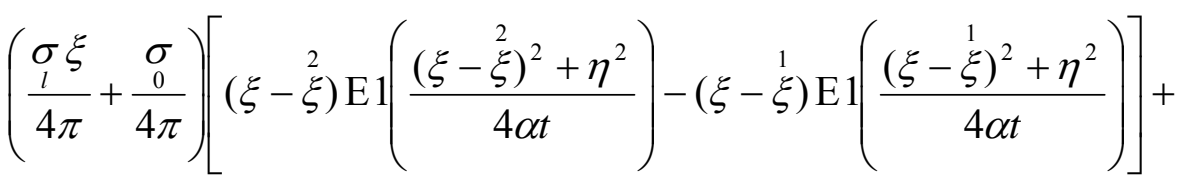

$$
\begin{aligned}
& \left(\sigma \xi \frac{|\eta|}{\sqrt{4 \pi}}+\frac{\sigma|\eta|}{\sqrt{4 \pi}}\right) \int_{\sqrt{\frac{\eta^{2}}{4 \alpha t}}}^{\infty} \exp \left(-w^{2}\right)\left[\operatorname{erfc}\left(w \frac{\xi-{ }^{2}}{|\eta|}\right)-\operatorname{erfc}\left(w \frac{\xi-\xi}{|\eta|}\right)\right] d w+ \\
& -\frac{1}{8 \pi}\left\{\left[\left(\xi-\stackrel{2}{\xi}^{2}\right)^{2}+\eta^{2}\right] \mathrm{E} 1\left(\frac{\left(\xi-\stackrel{2}{\xi}^{2}\right)^{2}+\eta^{2}}{4 \alpha t}\right)-\left[(\xi-\stackrel{1}{\xi})^{2}+\eta^{2}\right] \mathrm{E} 1\left(\frac{\left(\xi-\xi^{\prime}\right)^{2}+\eta^{2}}{4 \alpha t}\right)+\right. \\
& \left.-4 \alpha t\left[\exp \left(-\frac{\left(\xi-{ }^{\prime}\right)^{2}+\eta^{2}}{4 \alpha t}\right)-\exp \left(-\frac{\left(\xi-{ }^{2}\right)^{2}+\eta^{2}}{4 \alpha t}\right)\right]\right\}
\end{aligned}
$$

\subsubsection{Lago Poligonal de Ordem Dois e Grau Zero}

Os efeitos transientes produzidos por lago poligonal podem ser representados utilizando-se elementos linesink. Semelhantemente à formulação apresentada para os efeitos estacionários de um lago poligonal (seção 3.2.3), a sobreposição de $n$ linesinks produzirá $n+1$ incógnitas. Assim, a expressão do balanço hídrico obtida para uma linesink transiente pode ser empregada na formulação do potencial de descarga devido a um lago poligonal a fim de assegurar a integridade do balanço hídrico no lago em cada instante de tempo. O balanço hídrico em um elemento linesink de ordem dois, com esforços distribuídos conforme Eq. 3.3.21 torna-se expresso por:

$$
\int_{\frac{-L}{2}}^{\frac{L}{2}}\left(\sigma+\sigma_{l} \xi\right) d \xi=\sigma_{c} L
$$


O procedimento de solução é obtido conforme apresentado na seção 3.2.3, substituindose a função de influência do elemento linesink de regime estacionário Eq. 3.2.15 pela função de influência da equação Eq. 3.3.34. O balanço hídrico em cada instante, por sua vez, é feito de modo acumulativo pela seguinte expressão:

$$
\stackrel{k}{Q}=\sum_{c}^{k} \sigma_{j} L_{j}+\sum_{m=0}^{k-1} Q, \quad \text { para } k>0
$$

$$
\stackrel{k}{Q}=Q\left(t_{0}\right), \quad \text { para } k=0
$$

\subsubsection{Linedoublet de Ordem Dois e Grau Zero}

Zaadnoordijk (1988) desenvolve expressões para elementos linedoublets de ordem um utilizando-os apenas para obter expressões para a representação de areasinks. Os elementos linedoublets transientes de ordem dois podem ser aplicados diretamente em fronteiras onde podem ser especificadas diversas condições de escoamento como discutido na seção 3.4. Nesta seção, as expressões obtidas para linedoublets de ordem dois são obtidas.

O elemento linedoublet definido por Zaadnoordijk (1988) é obtido a partir da solução de Carslaw e Jaeger (1959) para o potencial devido a um pulso dipolar instantâneo. A notação segue a convenção definida por Zaadnoordijk (1988). A numeração no sobrescrito de $\Phi$ refere-se à ordem do polinômio utilizado na distribuição de esforços sobre o elemento. As letras utilizadas nos subscritos estão designadas como: ld referindo-se a linedoublet, $i$ ou o número 0 (zero) referindo-se respectivamente a instantâneo e grau zero e $l$ ou $q$ referindo-se a linear e quadrático, respectivamente.

Diferentemente dos elementos linesink, onde as cargas piezométricas são conhecidas nos extremos do elemento, em um linedoublet as cargas piezométricas são desconhecidas em qualquer ponto do elemento. Dessa forma, a distribuição de esforços deve assegurar que a distribuição de cargas piezométricas seja, além de contínua, suave (diferenciável) também nas extremidades do elemento (informação verbal de Schulz, 2004) ${ }^{10}$.

Considere-se que a distribuição de esforços sobre o elemento seja dada por um polinômio quadrático.

\footnotetext{
${ }^{10}$ Informação fornecida por Harry E. Schulz durante discussões cotidianas.
} 


$$
\lambda(\xi)=\underset{c}{\lambda}+\underset{l}{\lambda} \xi+\underset{q}{\lambda} \xi^{2}
$$

onde o subscrito $q$ refere-se ao parâmetro na forma quadrática. Os valores de Eq. 3.3.37 devem ser contínuos (coincidentes) nos nós de uma cadeia de elementos, observando-se que:

$$
\stackrel{i}{\lambda}\left(-\frac{L}{2}\right)=\stackrel{i-1}{\lambda}\left(\frac{L}{2}\right)=\stackrel{i}{c}_{c}^{i}-{ }_{l}^{i} L+\lambda_{q}^{i} \frac{L}{4}^{2}={ }_{c}^{i-1}+{\underset{l}{l}}_{l}^{i-1} L+\lambda_{q}^{i-1} \frac{L^{2}}{4}
$$

As inclinações de Eq. 3.3.37, da mesma forma, devem ser iguais nas extremidades de cada elemento a fim de representar a diferenciabilidade das cargas piezométricas $\phi$ em todas as direções. Diferenciando-se Eq. 3.3.37 em cada extremidade $\xi_{i}=L / 2$,

$$
\frac{d}{d \xi} \stackrel{i}{\lambda}\left(-\frac{L}{2}\right)=\frac{d}{d \xi} \stackrel{i-1}{\lambda}\left(\frac{L}{2}\right)=-\underset{l}{\lambda}+\underset{q}{\lambda} \frac{i}{2}={ }_{l}^{i-1}+\underset{q}{i-1} \frac{L}{2}
$$

Definida a aproximação da distribuição de esforços no elemento de acordo com a Eq. 3.3.37, o elemento linedoublet de segunda ordem, por sua vez, pode ser encontrado partindose dos resultados obtidos por Zaadnoordijk (1988). Conforme o autor, a expressão de dipolos instantâneos Eq. 3.3.5 com distribuição linear, orientados perpendicularmente à linha onde são somados, é obtida integrando a função:

$$
\underset{p d i}{\stackrel{1}{\Phi}}=\frac{\underset{l}{\lambda u+\underset{c}{\lambda}}}{8 \pi \alpha\left(t-t_{0}\right)^{2}} \eta \exp \left(-\frac{\left(\xi-\xi_{\xi}^{2}+\left(\eta-\eta^{2}\right)^{2}\right.}{4 \alpha\left(t-t_{0}\right)}\right)
$$

Efetuando-se a integral que define o potencial de um elemento de ordem um e grau zero tem-se:

$$
\underset{l d 0}{\Phi}=\int_{0}^{t} \int_{\substack{1 \\ \xi}}^{2} \underset{p d i}{\Phi} d u d \tau
$$


Após as correções de Barnes e Strack (2003) aplicadas sobre Zaadnoordijk (1988, Eq. 4.80), $\underset{\text { Id } 0}{1}$ deve ser dado por:

$$
\begin{aligned}
& \underset{\text { Id } 0}{\Phi}=(\lambda \underset{l}{\xi}+\underset{c}{\lambda}) \frac{1}{\sqrt{4 \pi}} \int_{\sqrt{\frac{\eta^{2}}{4 \alpha t}}}^{\infty} \exp \left(-w^{2}\right)\left[\operatorname{erfc}\left(w \frac{\xi-\xi}{|\eta|}\right)-\operatorname{erfc}\left(w \frac{\xi-\xi}{|\eta|}\right)\right] d w+ \\
& \underset{l}{\lambda} \frac{|\eta|}{4 \pi}\left[\operatorname{E} 1\left(\frac{\left(\xi-\xi^{2}+\eta^{2}\right.}{4 \alpha t}\right)-\mathrm{E} 1\left(\frac{\left(\xi-\xi^{2}\right)^{2}+\eta^{2}}{4 \alpha t}\right)\right]
\end{aligned}
$$

A expressão Eq. 3.3.42 fornece a solução para uma linedoublet de ordem um e grau zero. A integração da função de dipolo instantâneo Eq. 3.3.40 com a distribuição quadrática (Eq. 3.3.37) define o potencial de ordem dois e grau zero $(\stackrel{2}{\Phi})$ conforme a expressão:

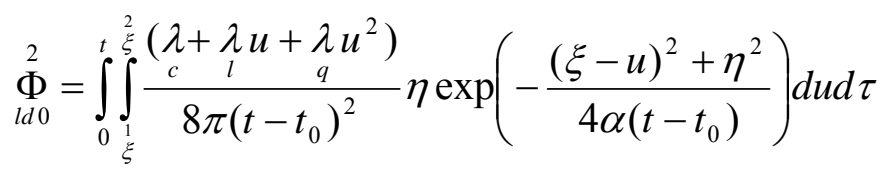

Observe-se que a expressão contém o potencial $\underset{\text { ld } 0}{\stackrel{1}{\Phi}}$ Eq. 3.3 .42 como parte de sua expressão, uma vez que $\underset{\text { ld } 0}{\stackrel{1}{9}}$ pode ser escrito como:

$$
\underset{I d 0}{\Phi}=\int_{0}^{t} \int_{\substack{\xi \\ \xi}}^{\frac{\lambda}{\xi}} \frac{\lambda u+\underset{c}{\lambda}}{8 \pi\left(t-t_{0}\right)^{2}} \eta \exp \left(-\frac{(\xi-u)^{2}+\eta^{2}}{4 \alpha\left(t-t_{0}\right)}\right) d u d \tau
$$

Assim, para obter-se $\stackrel{2}{\Phi}$, é necessário apenas resolver a integral de uma distribuição de esforços $\underset{q}{\lambda} u^{2}$ ao longo de um segmento de reta $\stackrel{\overline{\xi^{2}} \xi}{\xi}$. Separando-se as parcelas da soma presente no integrando e representando-se a integração da segunda parcela da integral por $\int_{0}^{t} \underset{\text { ldiq }}{\Phi} d \tau$, o potencial devido à distribuição complementar $\underset{q}{\lambda u^{2}}(\underset{\text { ldiq }}{\Phi})$ será dado por: 


$$
\begin{aligned}
& \underset{\text { ldiq }}{\Phi}=\underset{q}{\lambda} \exp \left(-\frac{\eta^{2}}{4 \alpha\left(t-t_{0}\right)}\right) \frac{\eta}{8 \pi \alpha\left(t-t_{0}\right)^{2}} \times
\end{aligned}
$$

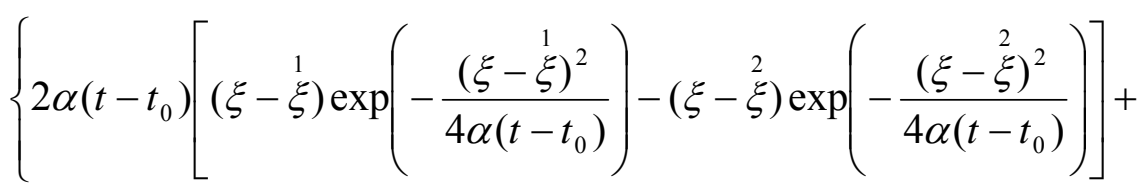

$$
\begin{aligned}
& \sqrt{\alpha \pi\left(t-t_{0}\right)}\left[2 \alpha\left(t-t_{0}\right)+\xi^{2}\left[\operatorname{erfc}\left(\frac{\xi-\xi^{2}}{\sqrt{4 \alpha\left(t-t_{0}\right)}}\right)-\operatorname{erfc}\left(\frac{\xi-\xi}{\sqrt{4 \alpha\left(t-t_{0}\right)}}\right)\right]\right\}
\end{aligned}
$$

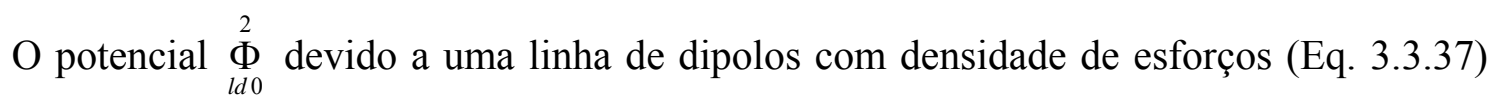
mantida constante no tempo, por sua vez, poderá ser obtido integrando-se agora $\underset{\text { ldiq }}{\Phi}$ em relação ao tempo. A partir de sua definição expressa em Eq. 3.3.43, $\underset{1 d 0}{\Phi}$ pode ser reescrito como:

$$
\stackrel{2}{\Phi}=\underset{I d 0}{\Phi}+\int_{\text {ld } 0}^{t} \underset{\text { ldiq }}{\Phi} d \tau
$$

e o termo complementar $\int_{0}^{t} \Phi d \tau$ ldiq $d$ será dado por:

$$
\begin{aligned}
& \int_{0}^{t} \underset{\text { Idiq }}{\Phi} d \tau=\underset{q}{\lambda} \frac{\eta}{4 \pi} \int_{0}^{t} \frac{1}{t-\tau}\left[\left(\xi-{ }^{1} \xi\right) \exp \left(-\frac{\left(\xi-\stackrel{1}{\xi}^{2}+\eta^{2}\right.}{4 \alpha(t-\tau)}\right)-\left(\xi \stackrel{2}{\xi}^{2}\right) \exp \left(-\frac{\left(\xi-\stackrel{2}{\xi}^{2}\right)^{2} \eta^{2}}{4 \alpha(t-\tau)}\right)\right] d \tau+ \\
& \lambda \frac{2 \alpha \eta}{8 \sqrt{\pi \alpha}} \int_{0}^{t} \frac{1}{\sqrt{t-\tau}} \exp \left(-\frac{\eta^{2}}{4 \alpha(t-\tau)}\right)\left[\operatorname{erfc}\left(\frac{\xi-\xi^{2}}{\sqrt{4 \alpha(t-\tau)}}\right)-\operatorname{erfc}\left(\frac{\xi-\xi}{\sqrt{4 \alpha(t-\tau)}}\right)\right] d \tau+ \\
& \lambda \xi_{q}^{2} \frac{\eta}{8 \sqrt{\pi \alpha}} \int_{0}^{t} \frac{1}{\sqrt{(t-\tau)^{3}}} \exp \left(-\frac{\eta^{2}}{4 \alpha(t-\tau)}\right)\left[\operatorname{erfc}\left(\frac{\xi-\xi^{2}}{\sqrt{4 \alpha(t-\tau)}}\right)-\operatorname{erfc}\left(\frac{\xi-\xi}{\sqrt{4 \alpha(t-\tau)}}\right)\right] d \tau
\end{aligned}
$$

Eq. 3.3.47

Mediante a mudança de variáveis: 


$$
u=\sqrt{\frac{\eta^{2}}{4 \alpha(t-\tau)}}, t-\tau=\frac{\eta^{2}}{4 \alpha u^{2}}, d \tau=\frac{\eta^{2}}{4 \alpha u^{3}} d u
$$

tem-se:

$$
\int_{0}^{t} \underset{\text { Idiq }}{\Phi} d \tau=\underset{q}{\lambda} \frac{\eta^{2}}{4 \sqrt{\pi}} I_{1}^{k}+\underset{q}{\lambda}(\xi-\xi) \frac{\eta}{2 \pi} \stackrel{k}{I}_{2}+\frac{\lambda \xi^{2}}{\sqrt{4 \pi}} \int_{\sqrt{\frac{\eta^{2}}{4 \alpha t}}}^{\infty} e^{-u^{2}}\left\{\operatorname{erfc}\left(u \frac{\xi-\xi_{\xi}^{2}}{\eta}\right)-\operatorname{erfc}\left(u \frac{\xi-\xi}{\eta}\right)\right\} d u
$$

Eq. 3.3.49

para $j=1,2$ e $k=0$, onde $\stackrel{k}{I_{1}}$ é dada por Eq. 3.3.18 e $\stackrel{k}{I}_{2}$ é dada em Zaadnoordijk (1988 Eq. 4.45) por:

$$
I_{2}^{k}=\int_{\sqrt{\frac{\eta^{2}}{4 \alpha t}}}^{\infty} \frac{1}{u^{2 k+1}} \exp \left(-u^{2} \frac{(\xi-\xi)^{2}+\eta^{2}}{\eta^{2}}\right) d u .
$$

As funções Eq. 3.3.18 e Eq. 3.3.50 são obtidas por Zaadnoordijk (1988, Apêndice E) conforme apresentado no Apêndice B deste estudo. Utilizando-se Eq. 3.3.18 e Eq. 3.3.50, a

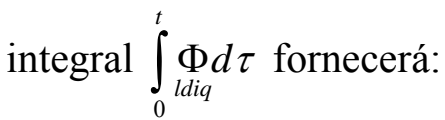




$$
\begin{aligned}
& \int_{0}^{t} \Phi_{\text {diq }}^{t} d \tau=\lambda \underset{q}{\lambda} \frac{\eta^{2}}{4 \sqrt{\pi}} \times\left\{\sqrt{\frac{4 \alpha t}{\eta^{2}}} e^{-\frac{\eta^{2}}{4 \alpha t}}\left[\operatorname{erfc}\left(\frac{\xi-\xi^{2}}{\eta} \sqrt{\frac{\eta^{2}}{4 \alpha t}}\right)-\operatorname{erfc}\left(\frac{\xi-\frac{1}{\xi}}{\eta} \sqrt{\frac{\eta^{2}}{4 \alpha t}}\right)\right]+\right. \\
& -\frac{1}{\sqrt{\pi}}\left[\frac{\xi-\stackrel{2}{\xi}}{\eta} \mathrm{E} 1\left(\frac{(\xi-\stackrel{2}{\xi})^{2}+\eta^{2}}{4 \alpha t}\right)-\frac{\xi-\stackrel{1}{\xi}}{\eta} \mathrm{E} 1\left(\frac{(\xi-\stackrel{1}{\xi})^{2}+\eta^{2}}{4 \alpha t}\right)\right]+ \\
& \left.-2 \int_{\sqrt{\frac{\eta^{2}}{4 a t}}}^{\infty} e^{-u^{2}}\left[\operatorname{erfc}\left(u \frac{\xi-\xi}{\eta}\right)-\operatorname{erfc}\left(u \frac{\xi-\xi}{\eta}\right)\right] d u\right\}+
\end{aligned}
$$

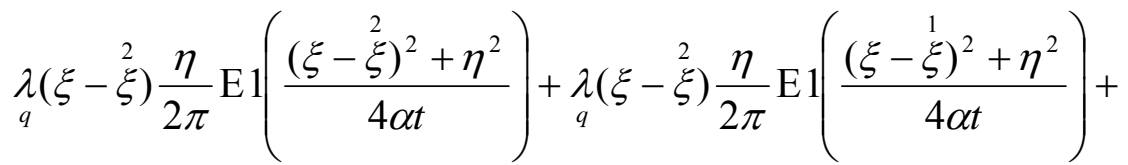

$$
\begin{aligned}
& \lambda_{q} \xi^{2} \frac{1}{\sqrt{4 \pi}} \int_{\sqrt{\frac{\eta^{2}}{4 \alpha t}}}^{\infty} e^{-u^{2}}\left[\operatorname{erfc}\left(u \frac{\xi-\xi}{\eta}\right)-\operatorname{erfc}\left(u \frac{\xi-\xi}{\eta}\right)\right] d u
\end{aligned}
$$

Assim, a expressão para o elemento de linedoublet ordem dois e grau zero $(\stackrel{2}{\underset{l d}{\infty}})$ pode, então, ser escrita a partir das equações Eq. 3.3.46 e Eq. 3.3.51 como:

$$
\begin{aligned}
& \stackrel{2}{\Phi}=\lambda \underset{q}{\lambda} \sqrt{\frac{\alpha t}{4 \pi}}_{e} e^{-\frac{\eta^{2}}{4 \alpha t}}\left[\operatorname{erfc}\left(\frac{\xi-\stackrel{\xi}{\xi}^{2}}{\eta} \sqrt{\frac{\eta^{2}}{4 \alpha t}}\right)-\operatorname{erfc}\left(\frac{\xi-\xi}{\eta} \sqrt{\frac{\eta^{2}}{4 \alpha t}}\right)\right]+ \\
& \frac{\underset{q}{\lambda} \eta}{4 \pi}\left[(\xi-\stackrel{2}{\xi}) \mathrm{E} 1\left(\frac{\left(\xi-\stackrel{2}{\xi}^{2}+\eta^{2}\right.}{4 \alpha t}\right)+3(\xi-\stackrel{1}{\xi}) \mathrm{E} 1\left(\frac{\left(\xi-\xi^{\prime}\right)^{2}+\eta^{2}}{4 \alpha t}\right)\right]+ \\
& \frac{\lambda\left(\xi^{2}-\eta^{2}\right)+\lambda \underset{l}{\xi}+\underset{c}{\lambda}}{\sqrt{4 \pi}} \int_{\sqrt{\frac{\eta^{2}}{4 \alpha t}}}^{\infty} e^{-u^{2}}\left[\operatorname{erfc}\left(u \frac{\xi-\stackrel{2}{\xi}^{2}}{\eta}\right)-\operatorname{erfc}\left(u \frac{\xi-\stackrel{1}{\xi}^{\prime}}{\eta}\right)\right] d u+ \\
& \lambda \frac{\eta}{4 \pi}\left[\mathrm{E} 1\left(\frac{\left(\xi-\xi^{\prime}\right)^{2}+\eta^{2}}{4 \alpha t}\right)-\mathrm{E} 1\left(\frac{\left(\xi-\xi^{2}\right)^{2}+\eta^{2}}{4 \alpha t}\right)\right]
\end{aligned}
$$

\subsubsection{Areasink Ordem Zero e Grau Zero}

Zaadnoordijk (1988) obtém uma expressão para o potencial devido a uma areasink com as mesmas propriedades geométricas que uma areasink estacionária definidas na seção 3.2.5. 
A expressão que define a solução para uma areasink (Eq. 3.2.40) pode ser reescrita da seguinte forma:

$$
\underset{a s}{\Phi}=\stackrel{a}{\Phi}+\stackrel{b}{\Phi}
$$

onde o subscrito as refere-se à areasink, $\stackrel{a}{\Phi}$ é igual a zero fora da área e $\stackrel{b}{\Phi}$ é igual ao potencial devido a uma determinada fonte fora da área.

A expressão que define a fonte pode ser arbitrada por uma distribuição de ordem um e grau um da seguinte forma:

$$
\stackrel{a}{\Phi}=\left\{\begin{array}{cl}
0, & \text { fora } \\
-\gamma \alpha t, & \text { dentro }
\end{array}\right.
$$

onde $\gamma$ é o esforço no elemento. Como condições de contorno, a descontinuidade do potencial $\stackrel{a}{\Phi}$ e da componente normal de seu gradiente são equacionadas através do contorno da área. Assim, um potencial $\stackrel{b}{\Phi}$ é definido pela sobreposição de linedoublets e linesinks ao longo dos lados da poligonal que cerca a área. Os esforços dos linedoublets são definidos de forma a se igualarem às diferenças do potencial $\stackrel{a}{\Phi}$ através de cada lado da poligonal. Da mesma forma, os esforços dos linesinks são definidos de maneira a eliminar as diferenças de gradiente do potencial através de cada lado. Assim, o potencial $\stackrel{b}{\Phi}$ pode ser expresso por Zaadnoordijk (1988) como:

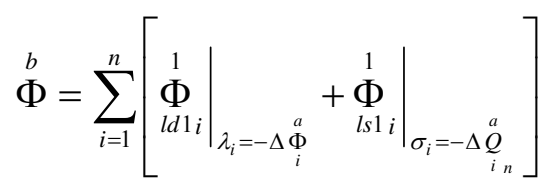

onde $\underset{i n}{Q}$ é a descarga normal devida ao potencial $\stackrel{a}{\Phi}$ no lado $i$ da poligonal, definida como positiva no sentido para fora da poligonal.

Entretanto, para uma fonte definida por um valor constante sobre toda a área na equação Eq. 3.3.54, $\stackrel{a}{\Phi}$ se torna constante, $\log 0, \stackrel{a}{Q}_{n}^{+}-\stackrel{a}{Q}_{n}^{-}=0$. Portanto, utilizando-se a definição do 
potencial $\stackrel{a}{\Phi}, \stackrel{a+}{\Phi}-\stackrel{a}{\Phi}=-\gamma \alpha t$, constante no espaço e linearmente variado no tempo, o potencial $\stackrel{a}{\Phi}$ será dado apenas por linedoublets com esforços conhecidos e iguais a $-\gamma \alpha \mathrm{t}$. Zaadnoordijk (1988, Eq. 4.89) apresenta a expressão para uma linedoublet de primeira ordem e grau um como:

$$
\begin{aligned}
& \underset{l d 1}{\Phi}=(\underset{c}{\lambda}+\underset{l}{\ln })\left\{\frac{\eta}{8 \pi}\left[(\xi-\stackrel{2}{\xi}) \mathrm{E} 1\left(\frac{\left(\xi-\stackrel{2}{\xi}^{2}+\eta^{2}\right.}{4 \alpha t}\right)-(\xi-\stackrel{1}{\xi}) \mathrm{E} 1\left(\frac{\left(\xi-\stackrel{1}{\xi}^{2}+\eta^{2}\right.}{4 \alpha t}\right)\right]+\right. \\
& \frac{1}{2 \pi}\left[\frac{\eta^{2}}{2 \alpha}+t\right] \int_{\sqrt{\frac{\eta^{2}}{4 \alpha t}}}^{\infty} e^{-u^{2}}\left[\operatorname{erfc}\left(u \frac{\xi-\stackrel{2}{\xi}^{2}}{\eta}\right)-\operatorname{erfc}\left(u \frac{\xi-\xi}{\eta}\right)\right] d u+ \\
& \left.-\frac{\eta \sqrt{t}}{4 \sqrt{\pi \alpha}} e^{-\frac{\eta^{2}}{4 \alpha t}}\left[\operatorname{erfc}\left(\frac{\xi-\xi}{\eta} \sqrt{\frac{\eta^{2}}{4 \alpha t}}\right)-\operatorname{erfc}\left(\frac{\xi-\xi}{\eta} \sqrt{\frac{\eta^{2}}{4 \alpha t}}\right)\right]\right\}+ \\
& -\lambda\left\{\frac{\eta}{4 \pi}\left[\frac{\left(\xi-\stackrel{2}{\xi}^{2}+\eta^{2}\right.}{2 \eta^{2}} \mathrm{E} 1\left(\frac{\left(\xi-\xi_{\xi}^{2}\right)^{2}+\eta^{2}}{4 \alpha t}\right)-\frac{\left(\xi-{ }^{\prime}\right)^{2}+\eta^{2}}{2 \eta^{2}} \mathrm{E} 1\left(\frac{\left(\xi-\xi^{2}\right)^{2}+\eta^{2}}{4 \alpha t}\right)\right]+\right. \\
& \left.-\frac{t \eta}{4 \pi}\left[\exp \left(-\frac{\left(\xi-\stackrel{2}{\xi}^{2}+\eta^{2}\right.}{4 \alpha t}\right)-\exp \left(-\frac{\left(\xi-\mathfrak{r}^{2}\right)^{2}+\eta^{2}}{4 \alpha t}\right)\right]\right\}
\end{aligned}
$$

Eq. 3.3.56

Fazendo $\underset{l}{\lambda=0}$ em $\quad$ Eq. 3.3.56, o potencial de descarga devida a $\stackrel{b}{\Phi}$ é dada em Zaadnoordijk (1988, Eq. 4.97) por:

$$
\begin{aligned}
& \stackrel{b}{\Phi}=\gamma \alpha \sum_{i=1}^{n}\left\{\frac{\eta_{i}}{8 \pi}\left[\left(\xi_{i}-\stackrel{2}{\xi}_{i}\right) \mathrm{E} 1\left(\frac{\left(\xi_{i}-\stackrel{2}{\xi}_{i}\right)^{2}+\eta_{i}^{2}}{4 \alpha t}\right)-(\xi-\stackrel{1}{\xi}) \mathrm{E} 1\left(\frac{\left(\xi_{i}-\stackrel{1}{\xi}_{i}\right)^{2}+\eta_{i}^{2}}{4 \alpha t}\right)\right]+\right. \\
& \frac{1}{2 \pi}\left[\frac{\eta_{i}^{2}}{2 \alpha}+t\right] \int_{\sqrt{\frac{\eta^{2}}{4 \alpha t}}}^{\infty} e^{-u^{2}}\left[\operatorname{erfc}\left(u \frac{\xi_{i}-\stackrel{2}{\xi}_{i}}{\eta}\right)-\operatorname{erfc}\left(u \frac{\xi_{i}-\stackrel{1}{\xi}_{i}}{\eta}\right)\right] d u+ \\
& \left.-\frac{\eta_{i} \sqrt{t}}{4 \sqrt{\pi \alpha}} e^{-\frac{\eta_{i}^{2}}{4 \alpha t}}\left[\operatorname{erfc}\left(\frac{\xi_{i}-\xi_{i}}{\eta} \sqrt{\frac{\eta_{i}^{2}}{4 \alpha t}}\right)-\operatorname{erfc}\left(\frac{\xi_{i}-\xi_{i}^{2}}{\eta} \sqrt{\frac{\eta_{i}^{2}}{4 \alpha t}}\right)\right]\right\}
\end{aligned}
$$


onde $\xi_{i}$ e $\eta_{i}$ são as coordenadas locais definidas sobre cada lado $i$ da poligonal. A expressão final do potencial devido a uma areasink ordem um e grau um é dada por (Zaadnoordijk, 1988, Eq. 4.98):

$$
\stackrel{1}{\Phi}=\left\{\begin{array}{cl}
\stackrel{b}{\Phi}, & \text { fora } \\
-\gamma \alpha t+\stackrel{b}{\Phi}, & \text { dentro }
\end{array}\right.
$$

\subsection{Condições de Contorno}

Uma expressão de uma condição de contorno é especificada apenas em pontos contidos nos contornos de forma que as distribuições de densidade de esforços presentes nos elementos sejam ajustadas. Assim, as condições de contorno são impostas em dois passos: no primeiro, uma função é escolhida para especificar o contorno e, no segundo, são escolhidos os pontos do contorno (pontos de controle) onde as distribuições de esforços utilizadas nos elementos serão ajustadas a fim de representar a condição ao longo de todo elemento (Figura 3.4.1).

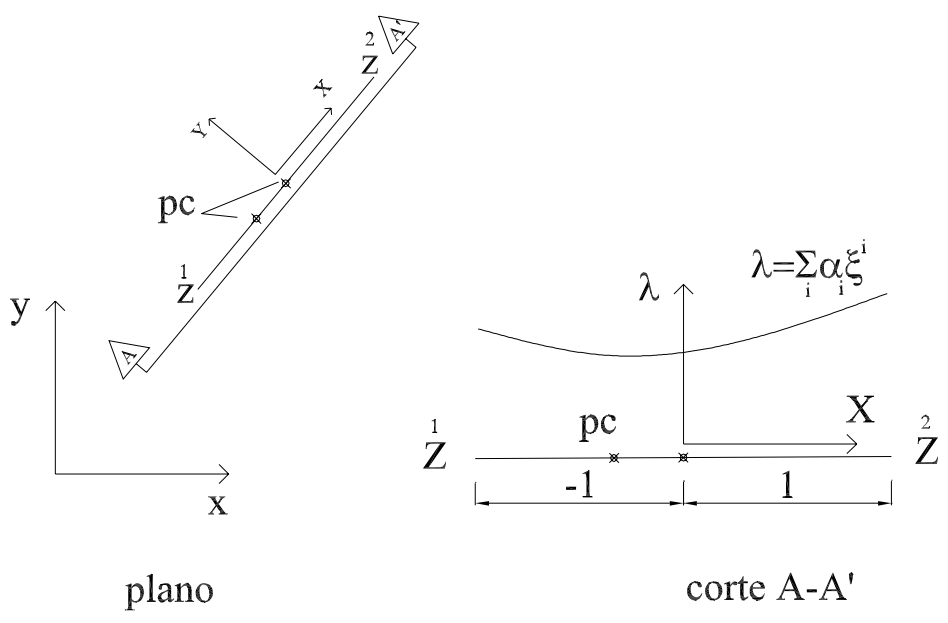

Figura 3.4.1 - Esboço da distribuição de esforços $(\lambda)$ sobre um elemento (pontos de controle indicados pelo símbolo pc)

Os parâmetros de distribuição de esforços utilizados nas funções que representam $\Phi, \Psi$ ou $Q$ de um elemento são os mesmos. Isso significa que, especificando-se uma condição de 
contorno, por exemplo, em termos de $\Phi$, a solução poderá ser expressa também em $\Psi$ ou Q. Ou seja, a especificação de condições de contorno em termos de uma variável de campo qualquer é válida como especificação para qualquer outra variável de campo. Isso permite que um modelo possua simultaneamente especificações de condições de contorno de diferentes naturezas.

Duas denominações podem ser distintas a princípio. Elementos cujos parâmetros são conhecidos são denominados "elementos especificados" (referidos pelo índice e) e os elementos cujos parâmetros são desconhecidos são denominados "elementos calculados" (referidos pelo índice c). Após a especificação da expressão de contorno em cada ponto desejado, os parâmetros desconhecidos de cada elemento são obtidos de maneira a satisfazer cada expressão especificada mediante a equação matricial:

$\mathbf{A} \lambda=\mathbf{B}$.

Eq. 3.4.1

onde $\boldsymbol{A}$ é a matriz de coeficientes, $\lambda$ é o vetor de incógnitas $\left(\lambda_{\mathrm{c}}\right)$ e $\boldsymbol{B}$, o vetor de termos independentes. A seguir, são discutidas diversas expressões utilizadas em contornos comumente encontrados em escoamentos subterrâneos. As condições impostas em cada espécie de contorno são expressas em termos da sobreposição de efeitos elementares (elementos analíticos). Dessa forma, cada linha da matriz de coeficientes reunirá as funções de influência daqueles elementos cujos parâmetros ainda não foram determinados constituindo-se uma matriz denominada "matriz cheia".

Deve-se notar que a constante de integração da solução do modelo $\left(C_{\text {modelo }}=\Sigma C_{e l}\right)$ deve ser determinada como uma incógnita do sistema. Assim, é especificado um ponto de referência no domínio onde um potencial de referência é arbitrado, longe o suficiente para que o comportamento do campo próximo aos contornos não seja influenciado pela escolha (ver seção 3.2.1).

\subsubsection{Potencial Conhecido}

As expressões especificadas nos contornos de um modelo de elementos analíticos são escritas aplicando-se o princípio da sobreposição de efeitos. Dado um ponto do domínio (pc) onde o valor do potencial $\Phi(\mathbf{p c})$ é conhecido, seu valor será expresso sobrepondo-se os efeitos de elementos especificados e elementos calculados. Os parâmetros dos elementos calculados 
serão obtidos de forma que o somatório dos potenciais produzidos por todos os elementos utilizados no modelo no ponto pc seja igual a $\Phi(\mathbf{p c})$. Assim,

$$
\Phi=\Phi_{\mathbf{p c}}
$$

ou ainda, expressando-se a sobreposição de efeitos:

$$
\sum_{c} \stackrel{c}{\Phi}=\Phi_{\mathbf{p c}}-\sum_{e} \stackrel{e}{\Phi}
$$

A especificação de potenciais é feita comumente em elementos linesink (ver seção 3.2.2). Assim, embora esteja especificando-se carga, o contorno analisado não caracteriza o primeiro tipo (Dirichlet), mas sim, um contorno do tipo Neumann (segundo tipo). Como discutido na equação Eq. 3.3.25, a distribuição de potenciais sobre uma linesink não depende da distribuição de esforços utilizada ao longo desses elementos e somente são asseguradas nos pontos de controle (símbolo pc na Figura 3.4.1).

\subsubsection{Estruturas Drenantes}

Fraturas, falhas ou outras estruturas drenantes que ocorrem em aqüíferos podem ser especificados pela descontinuidade da função de corrente entre os extremos do objeto. Pela definição da função de corrente (Eq. 2.1.14), a diferença entre as linhas de corrente nos extremos fornece a vazão que atravessa a direção longitudinal do objeto (Figura 3.4.2) da seguinte forma:

$$
\Psi^{+}-\Psi^{-}=-\stackrel{*}{Q_{s}}
$$

Considerando uma espessura $H^{*}$ e uma condutividade $K^{*}$ para o objeto, a partir da expressão do experimento de Darcy (Eq. 1.2.1), Strack (1989) obtém:

$$
\stackrel{Q}{Q}_{s}=-\frac{K^{*} H^{*}}{K} \frac{\partial \Phi}{\partial s}
$$




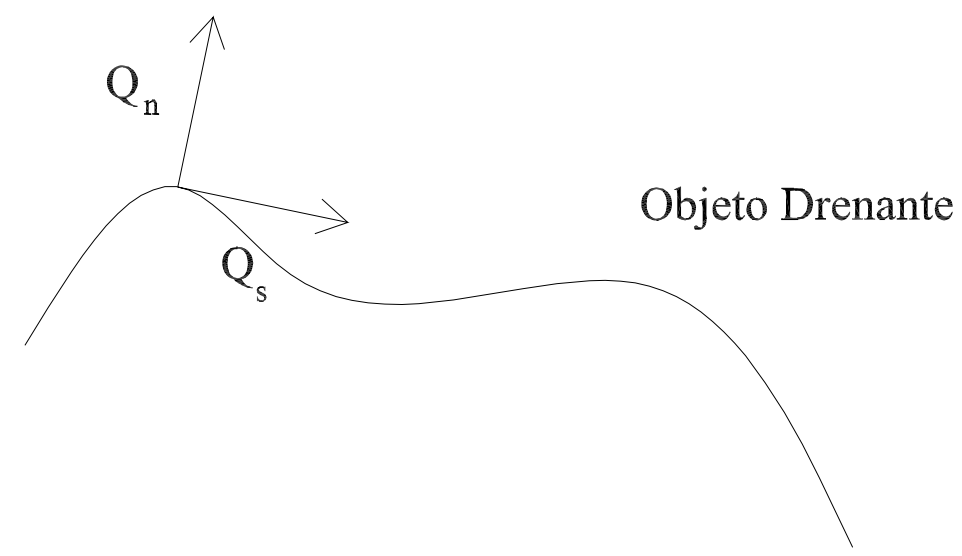

Figura 3.4.2 - Componentes de Vazão nas direções tangente e normal à estrutura.

O termo $\partial \Phi / \partial s$ refere-se à componente tangencial do gradiente do potencial imediatamente fora da estrutura. Dessa forma, pode-se definir uma componente de descarga tangencial e fora da estrutura $Q_{s}=-\partial \Phi / \partial s$ de maneira que $Q_{s}=-\mathfrak{R} \frac{2 d \Omega}{L d Z}$ (ver Figura 3.4.2). Assim, tem-se:

$$
Q_{s}=-\frac{K}{K^{*} H^{*}}\left(\Psi^{+}-\Psi^{-}\right)
$$

ou ainda,

$$
-\frac{K}{K^{*} H^{*}} \sum_{c}\left(\stackrel{c}{\Psi}^{+}-\stackrel{c}{\Psi}^{-}\right)-\sum_{c} \stackrel{c}{Q}_{s}=\frac{K}{K^{*} H^{*}} \sum_{e}\left(\stackrel{e}{\Psi}^{+}-\stackrel{e}{\Psi}^{-}\right)+\sum_{e} \stackrel{e}{Q}_{s}
$$

A condição de contorno torna-se completamente especificada distribuindo-se linedipoles, definidos pela expressão Eq. 3.2.5 multiplicada pelo número imaginário $i$.

\subsubsection{Vazão Específica Conhecida}


São discutidas duas aplicações da condição de contorno do segundo tipo (Neumann). Em casos de dreno ideal, a diferença de carga entre as extremidades do dreno é nula e conseqüentemente $Q_{s}=0$ e $\Psi^{+}=\Psi^{-}$Strack (1989) sugere:

$$
\Phi-\Phi_{A}=0
$$

ou ainda,

$$
\sum_{c} \stackrel{c}{\Phi}=\Phi_{A}-\sum_{e} \stackrel{e}{\Phi}
$$

A expressão exige a imposição de um potencial $\Phi_{A}$ em um dos extremos do dreno. Alternativamente, pode-se utilizar:

$$
Q_{s}=0
$$

ou seja,

$$
\sum_{c} \stackrel{c}{Q}_{s}=-\sum_{e}^{e} \stackrel{Q}{s}_{s}
$$

onde $Q_{s}$ é definida como na subseção anterior (3.4.2). A distribuição de esforços ao longo do contorno pode ser feita utilizando-se elementos linedipoles.

A segunda aplicação é a modelagem de paredes impermeáveis $Q_{n}=0$ (ver Figura 3.4.2), assim:

$$
\Psi-\Psi_{A}=0
$$

A expressão é sugerida em Strack (1989), embora haja advertências quanto a inconveniências e assim, o autor sugere ainda que expressão melhor utilizada no contorno então é:

$$
Q_{n}=0
$$


ou

$$
\sum_{c} \stackrel{c}{Q}_{n}=-\sum_{e} \stackrel{c}{Q}_{n}
$$

Semelhantemente à definição sugerida para $Q_{s}$ na subseção anterior, $Q_{n}$ pode ser definida, por sua vez como $Q_{n}=-\mathfrak{I} \frac{2 d \Omega}{L d Z}$.

\subsubsection{Condições de Contorno Combinadas}

A especificação de condições de contorno em regiões que definem porções do aqüífero com diferentes transmissividades é feita utilizando-se as condições do primeiro e do segundo tipo de forma combinada. As diferenças de propriedades podem ser exibidas na condutividade hidráulica, na espessura do aqüífero e na elevação da base do aqüífero. Para assegurar a continuidade do escoamento $Q$ e da carga piezométrica $\phi$, é necessário impor que:

$$
\phi^{+}=\phi^{-} \text {e } Q_{n}^{+}=Q_{n}^{-}
$$

Considerando a relação entre o potencial $\Phi$ e a carga $\phi$ na expressão Eq. 3.1.5a, uma fronteira entre diferentes condutividades pode ser escrita a partir da equação Eq. 3.4.15 em termos de potencial como:

$$
\Phi^{+} K^{-}=\Phi^{-} K^{+}
$$

ou

$$
K^{-} \sum_{c} \stackrel{c}{\Phi}^{+}-K^{+} \sum_{c} \stackrel{c}{\Phi}^{-}=-K^{-} \sum_{e} \stackrel{e}{\Phi}^{+}+K^{+} \sum_{e} \stackrel{e}{\Phi}^{-}
$$

A segunda imposição redunda em que:

$$
\Psi^{+}-\Psi^{-}=0
$$


A diferença de potencial exibida através do contorno pode ser representada empregando-se elementos linedoublets de segunda ordem impondo-se a condição Eq. 3.4.17 e que a distribuição de esforços tenha valores iguais (Eq. 3.3.38) e com a mesma inclinação (Eq. 3.3.39) nas extremidades do elemento. Observando que um elemento linedoublet não apresenta descontinuidade na componente $\Psi$ por definição, a segunda condição especificada será sempre satisfeita e os valores de seus esforços serão suficientemente especificados pela primeira condição.

Em aqüíferos confinados, a expressão Eq. 3.4.17 pode ser estendida para os casos de heterogeneidade na espessura $H$ e na cota da base $B$ da seguinte forma:

$$
\frac{\Phi^{+}+\frac{1}{2} K^{+} H^{2^{+}}}{K^{+} H^{+}}+B^{+}=\frac{\Phi^{-}+\frac{1}{2} K^{-} H^{2^{-}}}{K^{-} H^{-}}+B^{-},\left(\phi^{+}>H^{+} \text {e } \phi^{-}>H^{-}\right)
$$

ou

$$
\sum_{c} \frac{\stackrel{c}{\Phi}^{+}}{K^{+} H^{+}}-\sum_{c} \frac{\stackrel{c}{\Phi}^{-}}{K^{-} H^{-}}=-\sum_{e} \frac{\stackrel{e}{\Phi}^{+}}{K^{+} H^{+}}+\sum_{e} \frac{\stackrel{e}{\Phi}^{-}}{K^{-} H^{-}}+B^{-}-B^{+}+\frac{1}{2} H^{-}-\frac{1}{2} H^{+} \text {Eq. 3.4.20 }
$$

Em aqüíferos livres, a condição Eq. 3.4.15 redunda nas seguintes expressões nãolineares:

$$
\begin{aligned}
& \sqrt{\frac{2 \Phi^{+}}{K^{+}}}+B^{+}=\sqrt{\frac{2 \Phi^{-}}{K^{-}}}+B^{-}, \quad\left(\phi^{+}<H^{+} \text {e } \phi^{-}<H^{-}\right) \\
& \sqrt{\frac{2 \Phi^{+}}{K^{+}}}+B^{+}=\frac{\Phi^{-}+\frac{1}{2} K^{-} H^{2^{-}}}{K^{-} H^{-}}+B^{-}, \quad\left(\phi^{+}<H^{+} \text {e } \phi^{-}>H^{-}\right) \\
& \frac{\Phi^{+}+\frac{1}{2} K^{+} H^{2^{+}}}{K^{+} H^{+}}+B^{+}=\sqrt{\frac{2 \Phi^{-}}{K^{-}}}+B^{-}, \quad\left(\phi^{+}>H^{+} \text {e } \phi^{-}<H^{-}\right)
\end{aligned}
$$

Pode-se escrever a descontinuidade do potencial através da fronteira em cada ponto pc como: 


$$
\Phi_{\mathrm{pc}}^{+}-\Phi_{\mathrm{pc}}^{-}=\lambda_{\mathrm{pc}}=A_{\mathrm{pc}} \Phi_{\mathrm{pc}}^{-}
$$

onde $A_{i}$ é uma expressão obtida da equação Eq. 3.4.15, conforme cada um dos casos de heterogeneidade na espessura do aqüífero. Strack (1989, pág. 413) apresenta um processo iterativo para a determinação de $\lambda$ para os casos onde a equação Eq. 3.4.22 acarreta em expressões que dependem de $\phi$. Fazendo

$$
A_{\mathrm{pc}}=\frac{\Phi_{\mathrm{pc}}^{+}-\Phi_{\mathrm{pc}}^{-}}{\Phi_{\mathrm{pc}}^{-}}
$$

A equação Eq. 3.4.22 torna-se:

$$
\sum_{c} \stackrel{c}{\Phi}^{+}-\sum_{c} \stackrel{c}{\Phi}^{-}-A_{\mathrm{pc}} \sum_{c} \stackrel{c}{\Phi}^{-}=-\sum_{e} \stackrel{e}{\Phi}^{+}+\sum_{e} \stackrel{e}{\Phi}^{-}+A_{\mathrm{pc}} \sum_{e} \stackrel{e}{\Phi}^{-}
$$

O processo inicia-se com uma estimativa para $\Phi^{+}$e $\Phi^{-}$, por exemplo, negligenciando-se os parâmetros internos à região $\left(^{+}\right)$. A solução do sistema de equações fornece a próxima estimativa de $\lambda_{i}$ e conseqüentemente $A_{i}$, até que um critério de parada $\varepsilon$ seja atendido.

$$
\lambda_{\mathbf{p c}}=\Phi_{\mathbf{p c}}^{+}-\Phi_{\mathbf{p c}}^{-} \pm \varepsilon
$$

\subsubsection{Estruturas Semi-permeáveis}

Uma condição de contorno é classificada como do terceiro tipo (Cauchy) quando seu valor é dado em termos da variável dependente (e.g. $\phi$ - a carga piezométrica). Barragens subterrâneas e outros obstáculos semi-impermeáveis são exemplos de contornos do terceiro tipo. Escrevendo a expressão do experimento de Darcy (Eq. 1.2.1) através de uma parede delgada, obtém-se $Q_{n}($ ver Figura 3.4.2) da seguinte forma:

$$
Q_{n}=\frac{K}{K} \frac{K^{*} \frac{1}{2}\left\lfloor\phi^{+^{2}}-\phi^{-2}\right\rfloor}{b^{*}}=\frac{K^{*}}{K b^{*}}\left(\Phi^{+}-\Phi^{-}\right)
$$


Em aqüíferos confinados, escreve-se a mesma expressão da seguinte forma:

$$
Q_{n}=\frac{K}{K} \frac{K^{*} H\left[\phi^{+}-\phi^{-}\right]}{b^{*}}=\frac{K^{*}}{K b^{*}}\left(\Phi^{+}-\Phi^{-}\right) .
$$

Reescrevendo a expressão agora utilizando-se da sobreposição de efeitos, tem-se:

$$
\frac{K^{*}}{K b^{*}} \sum_{c}\left(\stackrel{c}{\Phi}^{+}-\stackrel{c}{\Phi}^{-}\right)-\stackrel{c}{Q},=-\frac{K^{*}}{K b^{*}} \sum_{e}\left(\stackrel{e}{\Phi}^{+}-\stackrel{e}{\Phi}^{-}\right)+\stackrel{e}{Q_{n}}
$$

onde $Q_{n}$ pode ser definida mais uma vez como $Q_{n}=-\mathfrak{I} \partial \Omega / \partial Z$.

Outra aplicação é encontrada em corpos de água cujos leitos sofreram colmatação. O coeficiente de drenança $\left(\gamma_{d}\right)$ relaciona as cargas piezométricas em torno da fronteira da seguinte maneira:

$$
\gamma_{d}=\left(\frac{\phi-\phi_{Y=0}}{c}\right)
$$

onde $c=\frac{H^{*}}{K^{*}}, \phi_{Y=0}$ é o nível de água superficial do corpo de água e $\phi$ é a carga piezométrica no aqüífero. O potencial em aqüíferos livres é uma função quadrática de $\phi$ (Eq. 3.4.26) e, portanto, o sistema de equações deixa de ser linear. Strack (1989) apresenta uma linearização da expressão do potencial, onde a matriz é resolvida em um processo iterativo (Strack, 1989 pág. 498, Mitchell-Bruker e Haitjema, 1996 e De Lange 1999).

\subsubsection{Condições Transientes}

Condições de contorno em escoamentos transientes podem ser impostas utilizando-se as convenções para as funções de influência e parâmetros de esforço definidas na seção 3.3.1 e a sobreposição de efeitos no tempo. As condições de contorno previamente discutidas podem ser estendidas para condições transientes escrevendo-se o potencial em cada intervalo de tempo. Assim, tomando-se, por exemplo, a imposição de um valor conhecido para o potencial, tem-se: 


$$
\Phi(\mathbf{p c}, t)=\stackrel{k}{\Phi}+\Phi_{s s}
$$

onde o índice $k$ refere-se ao $k$-ésimo instante de tempo (ou intervalo de tempo) no qual $\Phi(\mathbf{p c}, t)$ é obtido e $\Phi_{s S}$ refere-se a um modelo estacionário sobre o qual ocorrem os eventos transitórios. Valendo-se da sobreposição de efeitos, pode-se escrever:

$$
\sum_{c} \underset{c}{k}=\Phi(\mathbf{p c}, t)-\Phi_{s s}-\sum_{e} \underset{e}{k}-\sum_{e} \sum_{t t}^{k-1} \Phi_{e}^{t t}
$$

Observe-se que o potencial no $k$-ésimo instante $(\stackrel{k}{\Phi})$ é desmembrado em elementos calculados e elementos especificados e, em seguida, os elementos especificados são desmembrados novamente em elementos especificados no instante $k$ e elementos de intervalos anteriores a $k(t t<k-1)$. Deve-se observar, finalmente, que os parâmetros dos elementos são calculados apenas em um único instante de tempo $(k)$ e que apenas os elementos especificados em instantes anteriores ou iguais a $k$ estão "ativos", ou seja terão a função de influência não $\operatorname{nula}(\stackrel{0<t \leq k k}{\Lambda} j \neq 0)$

\subsection{Implementação Computacional}

O Método de Elementos Analíticos tem sido implementado em diversos programas computacionais. Nesta seção é feita uma discussão acerca do programa GNU $\operatorname{Tim}^{\mathrm{SL}}$ apresentado por Bakker et al. (2002) e distribuido sob os termos da licença LGPL (FSF, 1999), modificado para as necessidades deste trabalho. O código fonte do programa encontrase disponível no $\mathrm{CD}$ em anexo. O programa define três classes fundamentais de objetos que representam as expressões e variáveis definidas no método: classes de objetos geométricos, classes de objetos analíticos e classes de objetos aqüíferos. Cada objeto descreve propriedades e procedimentos característicos de seu respectivo tema.

O programa é escrito em Python (linguagem de programação interpretada e orientada com objetos), porém inclui rotinas escritas em Fortran (linguagem de grande difusão no meio científico). A comunicação entre as linguagens é feita por intermédio da compilação do código Fortran em um arquivo de vínculo dinâmico (.PYD). Python tem sido bastante utilizada 
em trabalhos científicos por fornecer uma sintaxe de alto nível e ainda ser interpretada com grande desempenho (e.g. Bakker e Strack, 2003).

Python é freqüentemente comparada a outras linguagens interpretadas como NewLisp e Perl. A terminologia utilizada em linguagens orientadas com objetos visa à associação com propriedades e processos observados na maioria dos objetos ao nosso redor. O projeto de cada tipo de objeto é referido como classe. A construção de cada exemplar é referida como instanciação. O comportamento de um determinado tipo de objeto é descrito em cada procedimento e propriedade definidos no contexto de sua classe. E, por fim, a extinção de um exemplar é referida como destruição. O ciclo compreendido entre a construção e a destruição de um objeto é chamado ciclo de vida. Linguagens orientadas com objetos oferecem poucas regras de sintaxe e grande modularidade, conseqüentemente, grande legibilidade e redução do código.

\subsubsection{Classes de Objetos Geométricos}

A primeira classe fundamental é a classe de objetos geométricos: Geometry2d. A classe Geometry2d é especificada de modo a representar os conceitos que definem uma geometria plana no programa. Nesta classe estão definidos apenas os nomes dos procedimentos e os critérios de verificação da integridade de suas entradas. Cada forma geométrica é derivada a partir da definição de geometria feita na classe Geometry2d. A extensão ou derivação de conceitos ou espécies (classes) nas linguagens orientadas com objetos é feita por intermédio de heranças. A principal vantagem da passagem de heranças para uma classe é evitar a necessidade de redundâncias no programa. Assim, as instruções peculiares a cada forma geométrica são "sobre-escritas" às definições feitas na classe. Se ocorrerem propriedades ou

procedimentos idênticos aos da classe primitiva, estes podem ser recuperados diretamente a partir da classe derivada. Em cada geometria são definidas, por exemplo, as transformações de coordenadas, cálculos como comprimento e área, formatação de saída para arquivos (.BLN) etc. Na Figura 3.5.1 é apresentado o diagrama de herança das classes especificadas no programa. A figura é constituída de três listas de blocos que contêm o nome da classe especificada. No topo de cada lista encontra-se o nome da respectiva classe fundamental de onde partem setas que apontam na direção das classes que recebem sua herança. As classes de objetos geométricos são encontradas na primeira coluna apresentada na Figura 3.5.1. 


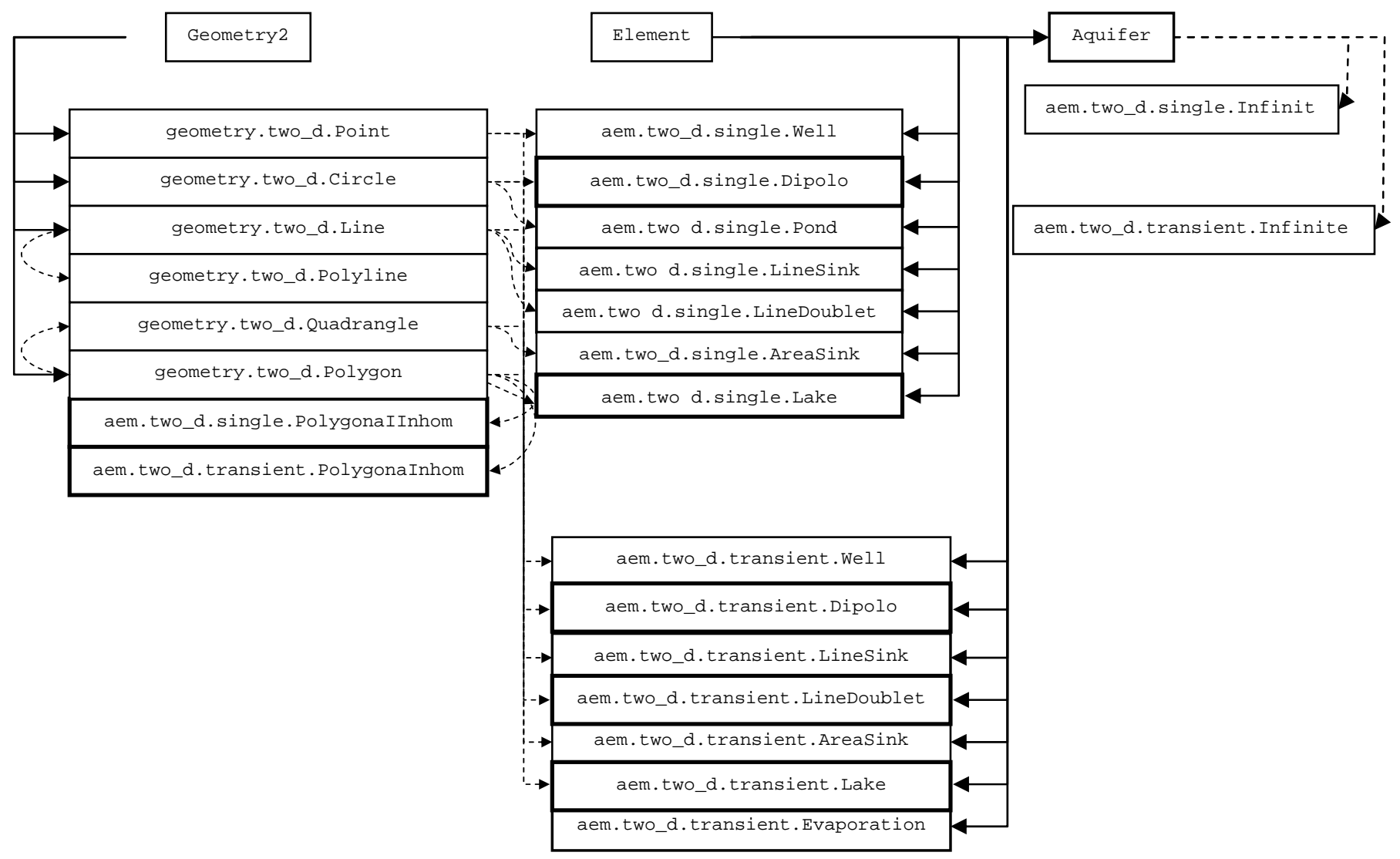

Figura 3.5.1 - Estrutura de heranças entre as classes do programa. Blocos no topo de cada lista designam classes fundamentais. Setas indicam a direção da herança. Linhas contínuas e tracejadas indicam respectivamente primeiro e segundo grau das heranças. 


\subsubsection{Classes de Objetos Analíticos}

Elementos estacionários e transientes são representados no programa por objetos analíticos. Para isso, são necessárias representações geométricas e físicas do comportamento dos elementos. As propriedades geométricas observadas em cada tipo de elemento são encontradas nas classes derivadas de Geometry2d. Assim, uma classe de objetos analíticos poderá ser especificada no programa derivando-se definições feitas em duas classes fundamentais: Geometry2d e Element. A classe Element, portanto, será responsável pela definição apenas de propriedades e procedimentos que descrevem o comportamento físico do elemento. Na segunda lista de blocos da Figura 3.5.1 apresenta-se a estrutura de heranças utilizada nas classes de objetos analíticos do programa.

As propriedades do meio, assim como condutividade hidráulica, porosidade etc., e efeitos regionais, como campo preferencial, potencial de referência etc., são representados no programa por intermédio de classes de objetos aqüíferos (Aquifer). Os objetos aqüíferos não possuem propriedades geométricas, porém desempenham influência física sobre os demais elementos, portanto, são representados no programa por classes derivadas apenas a partir de Element (ver terceira lista de blocos na Figura 3.5.1). São os objetos Aquifer que definem parâmetros e descrevem procedimentos que gerenciam elementos e variáveis no modelo.

\subsubsection{Classificação dos Procedimentos}

Tanto classes de objetos analíticos (representativos dos elementos) como as classes de objetos aqüíferos são especificadas de forma a incluir procedimentos do tipo: a) procedimentos de construção, b) procedimentos de influência, c) procedimentos de contribuição, d) procedimentos de acumulação e e) procedimentos de solução. A seguir é discutido cada tipo de procedimento.

a) Procedimentos de Construção

Os procedimentos construtores são responsáveis pela definição das propriedades de um objeto. Algumas propriedades são comuns a qualquer objeto analítico. A primeira propriedade comum é a lista de elementos presentes no modelo, chamada elementList. Essa lista contém pelo menos o próprio objeto. Cada objeto derivado de Element possui ainda um parent, uma propriedade que armazena qual o objeto que ocupa a primeira posição na elementList. A 
propriedade elementList é melhor explicada na discussão dos procedimentos de solução. A última propriedade de qualquer objeto Element é chamada parameters. A propriedade parameters é definida como um vetor que contém os parâmetros da distribuição de densidade de esforços utilizados na expressão do elemento representado. Assim, qualquer expressão definida para um elemento (seja ela uma expressão para $\Phi, \Psi$ ou $\mathbf{Q}$ ) é computada como o produto escalar do vetor parameters pelo vetor de termos de influência (ver Eq. 3.2.4).

Os argumentos utilizados nos procedimentos de construção dos elementos, a menos dos aqüíferos, estão padronizados de forma a receber: um objeto geométrico, uma lista de parâmetros e o objeto aqüífero. A lista de parâmetros, no entanto, é atribuída à propriedade parameters somente para objetos onde é conhecido o parâmetro a priori. Por exemplo, poços cuja operação é conhecida, ou elementos areasink representativos de áreas cujas taxas de recargas são conhecidas, e assim por diante. Elementos cujos parâmetros são incógnitas são derivados dos elementos de parâmetros pré-determinados, de forma que a lista de parâmetros passada como argumento para a construção recebe valores de campo a serem especificados como condições de contorno. Por exemplo, poços onde a operação não é conhecida, mas seus potenciais sim, devem ser construídos (instanciados) para seu determinado valor de potencial. Assim, os parâmetros de cada poço são determinados a partir da solução do modelo de modo a satisfazer o valor de potencial atribuído ao poço. Portanto, um objeto analítico é definido no programa não apenas pelos seus procedimentos de influência, mas também pelas suas condições de contorno.

O Método de Elementos Analíticos é concebido de modo que é necessário especificar um potencial de referência. Como já discutido, o potencial de referência é imposto ao modelo em um ponto de referência para que a soma das constantes de integração de cada elemento (ver seção 3.2.1) seja determinada. Uma vez que o objeto aqüífero é concebido para gerenciar elementos no modelo, o potencial de referência é um dos parâmetros (parameters) deste objeto.

b) Procedimentos de Influência

Os procedimentos de influência especificam o tipo de efeito a ser analisado. Três tipos de procedimentos de influência podem ser encontrados: potentialinfluence, streamInfluence e dischargeInfluence. Cada tipo de procedimento representa, 
respectivamente: as funções de influência do potencial, as funções de influência das linhas de corrente, as funções de influência do vetor de descarga. A saída de cada função é especificada de forma a retornar um vetor contendo os $n$ termos da função que devem ser multiplicados por cada um dos $n$ parâmetros de esforços previstos na expressão do elemento (ver Eq. 3.2.4).

c) Procedimentos de Contribuição

Os procedimentos de contribuição são responsáveis pelo cômputo individual do efeito desejado. Ou seja, é o procedimento responsável pela multiplicação do vetor parameters pelo vetor obtido de um procedimento de influência. Assim, os procedimentos de contribuição têm seu nome baseado na variável de campo estudada: potentialcontribution, streamContribution e dischargecontribution.

d) Procedimentos de Acumulação

Os procedimentos de acumulação totalizam as contribuições individuais dos efeitos desejados. Os procedimentos de acumulação são especificados para cada variável de campo que se deseja analisar. Esses métodos são responsáveis pela sobreposição dos efeitos de cada elemento no modelo. Assim, os procedimentos de acumulação não dependem do elemento e são especificados no contexto da classe que gerencia o modelo: Aquifer. Os procedimentos de acumulação, por sua vez, também são baseados no nome das variáveis de campo: potential, discharge, stream, waterBalance e trace, que acumula o deslocamento de uma partícula imaginária lançada no aqüífero a partir de uma determinada posição.

Originalmente, o programa $\operatorname{Tim}^{\mathrm{SL}}$ (versão 0.3 ) não prevê o procedimento de acumulação waterBalance. A necessidade de implementar esse procedimento surge quando da implementação do elemento lago poligonal (ver seção 3.2.3 e 3.3.3). Ambos, lago poligonal e funções de balanço hídrico, estão inseridos no programa $\operatorname{Tim}^{\mathrm{SL}}$ em decorrência das particularidades apresentadas ao longo do presente trabalho. Assim, neste trabalho, o balanço hídrico é implementado no programa computacional por intermédio dos seguintes procedimentos: waterBalance, waterBalanceContribution e waterBalanceInfluence, classificados respectivamente como procedimento de acumulação, procedimento de contribuição e procedimento de influência.

e) Procedimentos de Solução 
Este grupo de procedimentos implementa a construção do sistema de equações, sua solução e a obtenção dos parâmetros de cada elemento (parameters) a partir da solução do sistema. Os processos de forma geral são versáteis, incluindo a possibilidade de manipulação de quantidades arbitrárias de pontos de controle e permitindo a sobre-especificação dos elementos. São especificados quatro tipos de procedimentos de solução no programa: o gerenciador do sistema (solver), a obtenção dos termos da matriz de coeficientes (getMatrixCoefficients), a obtenção de linhas para a matriz e para o vetor de termos independentes (getMatrixContribution) e a atribuição dos resultados para os devidos parâmetros dos elementos (getParameters), discutidas respectivamente a seguir.

A matriz de coeficientes produzida em um modelo tem o número de colunas igual ao número de parâmetros desconhecidos nos elementos contidos na elementList do elemento eleito como parent do modelo. O processo de construção da matriz utiliza o procedimento getMatrixContribution de cada elemento. O processo é invocado do interior do procedimento solve. Assim, o procedimento getMatrixContribution é sobre-escrito apenas para as classes daqueles objetos que não possuem parâmetros conhecidos a priori. $\mathrm{O}$ procedimento getMatrixContribution dos elementos pré-determinados não retorna qualquer valor. A saída dos procedimentos contém uma linha de ambos, da matriz de coeficientes e do vetor de termos independentes, para cada ponto de controle definido para o elemento.

Como visto na seção 3.4, cada linha da matriz de coeficientes deve ser escrita de forma a especificar uma condição de contorno. Assim, os termos da matriz expressam o somatório das funções de influência dos elementos não predeterminados. Por outro lado, diferentemente das expressões da seção 3.4, os termos do vetor independente expressam o valor especificado no contorno menos o somatório dos efeitos de todos os elementos, incluindo aquele do qual é requisitado o procedimento getMatrixContribution. Portanto, a solução obtida fornece a diferença dos parâmetros antes e depois da solução do sistema.

Como o número de linhas na matriz de coeficientes é igual à quantidade de pontos de controle utilizada em todos os elementos, adotando-se o número de pontos de controle igual ao número de parâmetros desconhecidos obtém-se uma matriz quadrada. Se o número de pontos de controle é maior que o número de incógnitas, a matriz passa a ter mais linhas que colunas, o que consiste na sobre-especificação do problema. No entanto, a solução da matriz, neste caso, não pode ser obtida de forma exata. Assim, o objetivo do procedimento solve é 
percorrer toda lista de elementos, incluindo os de parâmetros pré-determinados, resultando no preenchimento da matriz de coeficientes e no vetor de termos independentes. Montado o sistema, é utilizado um pacote numérico de álgebra linear para a obtenção da solução.

O procedimento getMatrixCoefficient, da mesma forma que os demais tipos de procedimentos discutidos até aqui, é único para cada elemento. A necessidade de um procedimento exclusivo para a obtenção de valores de coeficientes torna-se evidente em objetos onde é necessário incluir como incógnita do sistema algum termo além daqueles presentes no procedimento de influência. Por exemplo, o caso estudado na seção 3.2.1, onde é estudado um lago sujeito a um escoamento uniforme. Identifica-se no exemplo três objetos analíticos: o aqüífero, o poço e o dipolo. Somente o poço possui o parâmetro pré-determinado. Embora sejam conhecidos a descarga preferencial (uniforme) do aqüífero e seu potencial de referência, é necessário definir uma incógnita para obter o valor da soma das constantes de integração de todos os efeitos. Assim, a função de influência do aqǘfero possui dois termos conhecidos e um desconhecido. Da mesma forma, a função de influência do dipolo possui dois parâmetros, porém seu potencial é desconhecido. Assim, observa-se que cada elemento, além de possuir um procedimento de influência, deve também possuir um procedimento de representação de suas incógnitas no sistema.

Essa representação é implementada, então, utilizando-se a estrutura lambda (recurso disponível em Python e em outras linguagens de alto nível). A estrutura lambda permite a definição temporária de procedimentos, que pode ser passada para um outro procedimento como se fosse um argumento. Assim, o procedimento getMatrixCoefficient recebe como argumento o procedimento de influência desejado e retorna os termos que estão sendo requisitados como incógnitas do sistema.

O procedimento getParameters é especificado de modo semelhante a getMatrixCoefficient. A necessidade de especificação do procedimento para cada objeto torna-se da mesma forma evidente diante de situações onde os termos da função de influência não expressam necessariamente as incógnitas do elemento. Assim, fazendo o processo inverso de getMatrixCoefficient, o procedimento tem o objetivo de identificar, dentre as incógnitas resolvidas, quais devem ser atribuídas à propriedade parameters do objeto.

\subsubsection{Objetos Analíticos Transientes}

O pacote de simulações transientes no $\operatorname{Tim}^{\mathrm{SL}}$ realiza o cálculo do campo de transitórios em águas subterrâneas a partir de uma solução estacionária inicial. A construção dos 
elementos transientes é feita, da mesma forma que os elementos estacionários, a partir de uma geometria, uma lista de parâmetros e um parent. Entretanto, os parâmetros dos elementos transientes devem permitir variação temporal e, portanto, sua lista contém pares de valores, o primeiro assinalando o instante de tempo e o segundo, o valor do parâmetro. Alguns elementos, à semelhança dos elementos estacionários, também podem ter seus valores prédefinidos, e.g. poços e areasinks. Os instantes onde são assinalados os valores indicam apenas o momento em que ocorre a mudança do valor do parâmetro. Os valores, por sua vez, são mantidos constantes até o instante do próximo evento. Outros elementos devem receber especificações de valores de campo, e.g. carga, para que sejam especificadas as condições de contorno no processo de solução. Os instantes de tempo devem ser ordenados de forma crescente e iniciar no instante zero, para o qual é obtido o modelo estacionário.

Uma outra propriedade importante é o initialsteadystate. Essa propriedade caracteriza o objeto correspondente à condição inicial de um elemento transiente. Durante o processo de construção dos elementos transientes, os respectivos objetos estacionários são também invocados e atribuídos à variável initialsteadystate. Não obstante essa relação entre elementos transientes e estacionários, estes últimos não participam da lista de elementos (elementList) do primeiro. A lista de elementos de um elemento transiente é especificada de modo a conter um objeto da classe Unitstep para cada mudança obtida para os parâmetros dos elementos durante a simulação. Assim, para cada instante de tempo em que o processo de solução é acionado, um elemento UnitStep é construído a partir da herança do elemento que o requisitou. A variável elementlist dos elementos transientes, diferentemente dos elementos estacionários, não contém o próprio elemento.

Os objetos aqüíferos do pacote de simulações transientes são especificados de modo a incluírem propriedades como coeficiente de armazenamento, espessura média (para aqüíferos livres) e difusividade, além dos métodos já considerados no aqüífero estacionário. Sua lista de elementos (elementList), ao contrário dos demais elementos transientes, contém o próprio objeto. Os parâmetros contidos em parameters não armazenam os valores da lista de parâmetros passada para o processo de construção do elemento. Em modelos transientes, à semelhança das classes de elementos estacionários, elementos transientes pré-determinados têm sua lista de parâmetros (parameters) especificada de forma a conter as diferenças entre os resultados ao longo do intervalo de tempo entre cada evento.

Devido à formulação dos elementos ser em termos de distribuição de esforços, uma evolução do campo de escoamento onde utiliza-se elementos pré-determinados é exata 
quando o contorno representado exibir valores de esforços constantes ao longo de determinados intervalos de tempo. Considere-se a situação onde os esforços de apenas uma linesink sofrem um acréscimo instantâneo no instante inicial da simulação. A variação do esforço é ilustrada na Figura 3.5.2 e a variação da carga piezométrica sobre um dos pontos de controle $(p c)$ da linesink e em um ponto qualquer fora do elemento, por sua vez, é ilustrada na Figura 3.5.3. Devido à formulação do elemento, tem-se que a variação da carga piezométrica sobre o elemento é exata para as condições apresentadas. Isso ocorre por que os elementos são concebidos matematicamente para expressar variações de parâmetros que se mantêm constantes ao longo do tempo.

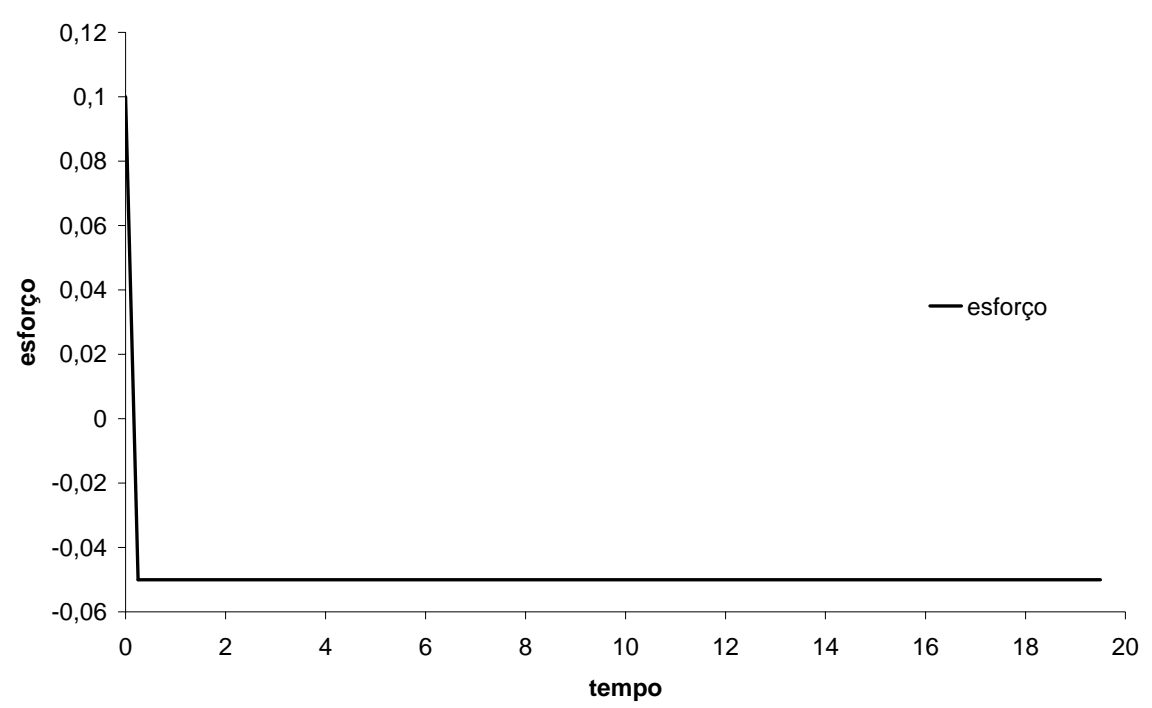

Figura 3.5.2 - Parâmetros pré-determinados constantes ao longo do tempo 


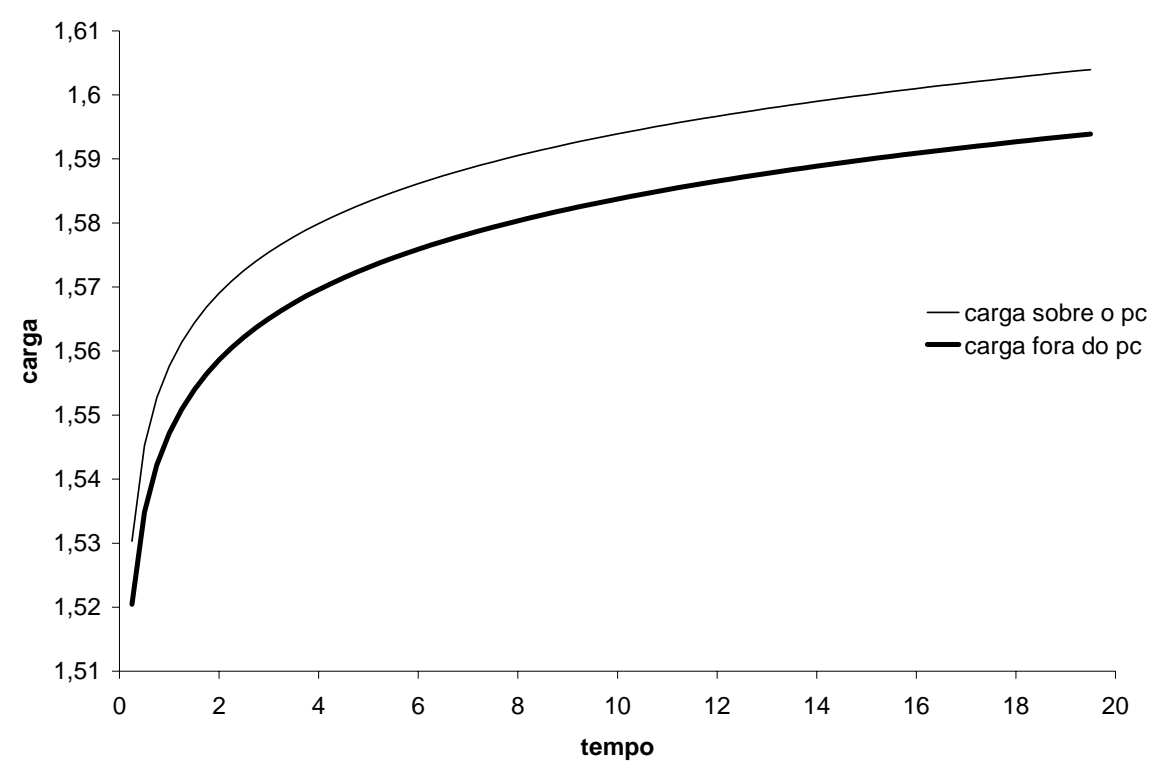

Figura 3.5.3 - Evolução da carga piezométrica em um ponto posicionado sobre o elemento determinadas a partir de esforços pré-determinados

Por outro lado, os elementos não pré-determinados, que especificam condições de contorno ao longo do tempo, devem ter os valores de seus esforços obtidos em pequenos intervalos de tempo de modo a descrever a evolução desses parâmetros utilizando-se valores constantes em cada intervalo. Uma vez que é atribuída uma variação, não mais do esforço para o qual o elemento foi concebido, mas uma variação de uma variável do campo, a evolução do esforço é aproximada a partir das soluções obtidas para os esforços nos elementos em cada intervalo de tempo subseqüentemente. Considere o caso onde a carga piezométrica no início da simulação é unitária e no instante seguinte vale 1,5 L (ver Figura 3.5.4). A evolução dos esforços obtidos para que sejam atendidas as condições impostas sobre o elemento é ilustrada na Figura 3.5.5. 


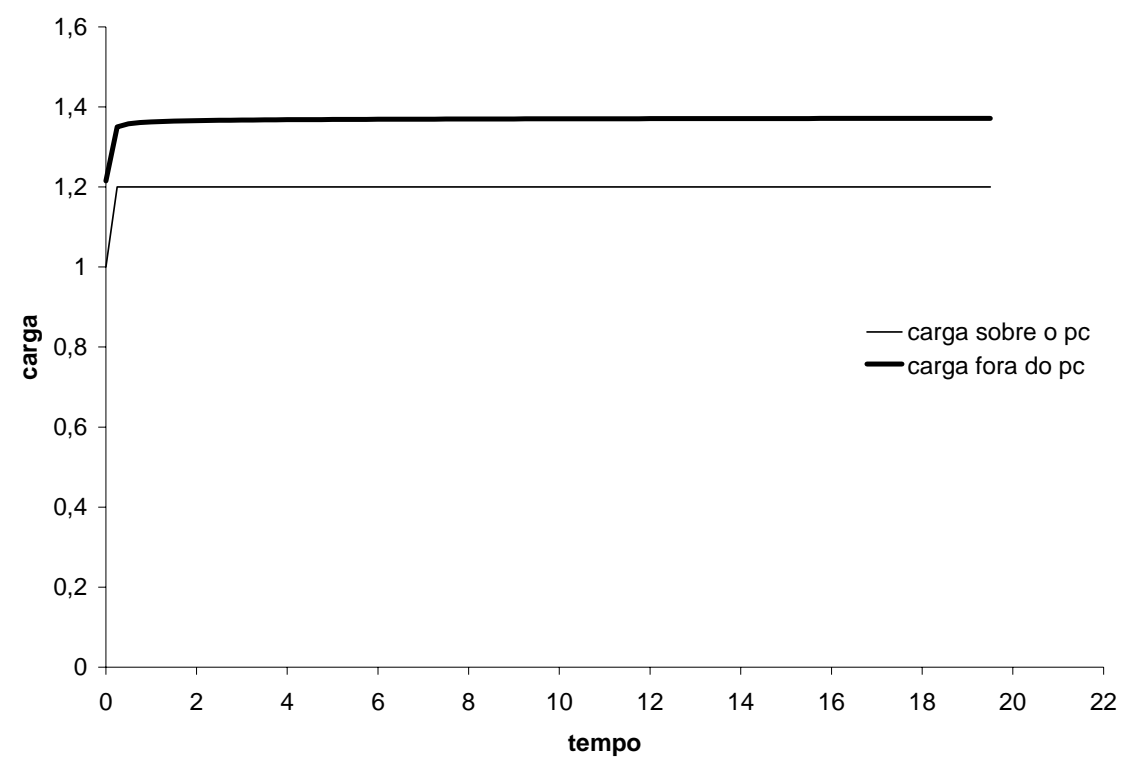

Figura 3.5.4 - Variação pré-determinada da carga piezométrica sobre o elemento e variação da carga em um ponto posicionado fora do elemento

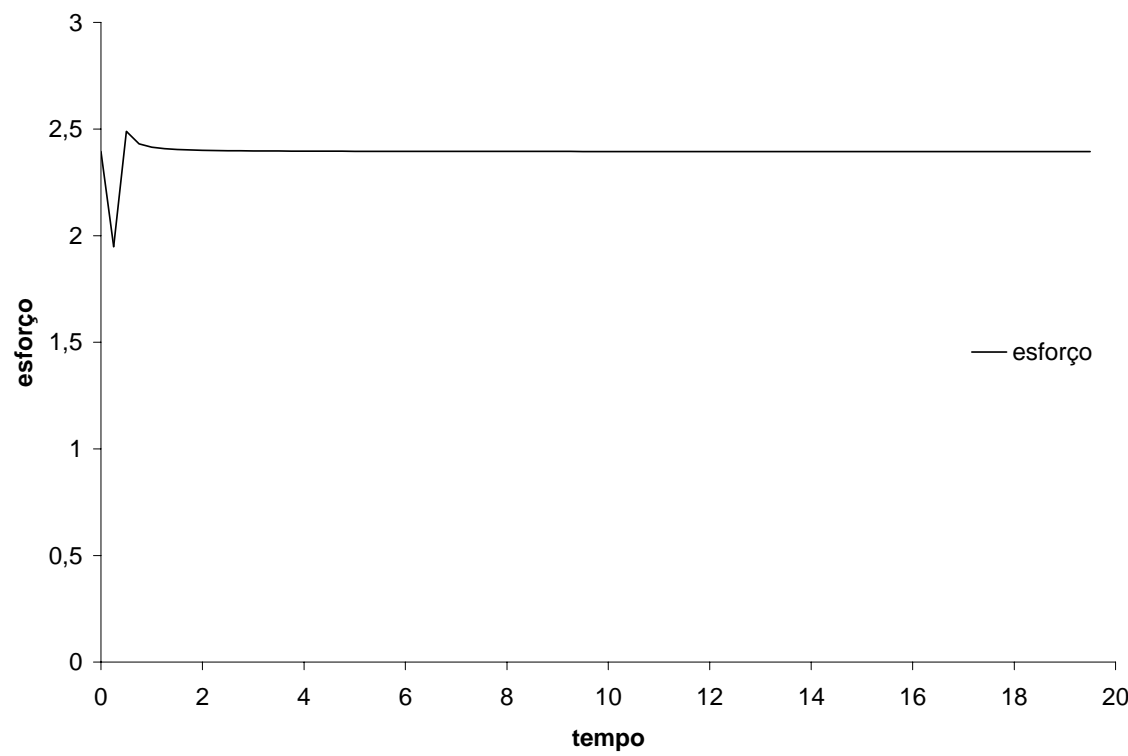

Figura 3.5.5 - Evolução de parâmetros determinados a partir de cargas piezométricas pré-determinadas sobre o elemento 


\subsubsection{Avaliação da integral de Rosser}

Embora a formulação proposta por Zaadnoordijk (1988) seja elegante, a avaliação da integral da Eq. 3.3.20 (chamada de integral de Rosser) por intermédio das expansões de séries propostas por Litkouhi e Becker (1982 apud de Barnes e Strack, 2003) ${ }^{11}$ converge lentamente quando $\frac{\xi-\xi_{j}}{|\eta|} \approx 1$. Recentemente, Barnes e Strack (2003) discutiram a utilização de funções que produzem uma convergência mais rápida na avaliação da integral da Eq. 3.3.20 quando $\frac{\xi-\xi_{j}}{|\eta|} \approx 1$. Barnes e Strack (2003) sugerem arbitrariamente os limites 0,6 e 1,3 como limites inferior e superior para a definição de o que significa $\frac{\xi-\xi_{j}}{|\eta|} \approx 1$. A avaliação proposta por Barnes e Strack (2003) é vantajosa em grande parte do domínio do plano $\left(\sqrt{\frac{\eta^{2}}{4 \alpha t}} \frac{\xi-\xi_{j}}{|\eta|}, \frac{|\eta|}{\xi-\xi_{j}}\right)$ (ver Figura 3.5.6, gráfico à esquerda). No entanto, observa-se neste trabalho, que quando $\sqrt{\frac{\eta^{2}}{4 \alpha t}} \frac{\xi-\xi_{j}}{|\eta|}<1$ a avaliação de Barnes e Strack (2003) exibe descontinuidades nos limites de $\frac{|\eta|}{\xi-\xi_{j}} \approx 1$, qualquer que tenha sido a definição adotada para os limites superior e inferior dos valores de $\frac{|\eta|}{\xi}$ próximos a 1,0 (ver Figura 3.5.6, gráfico $\xi-\xi_{j}$

à direita).

${ }^{11}$ Litkouhi e Becker (1982) Temperatures in Semi-Infinite Body Heated by Constant Heat Flux Over Half Space. In: Proceedings of the Seventh International Heat Transfer Conference Muenchen, v2, pp21-27, Washington DC 

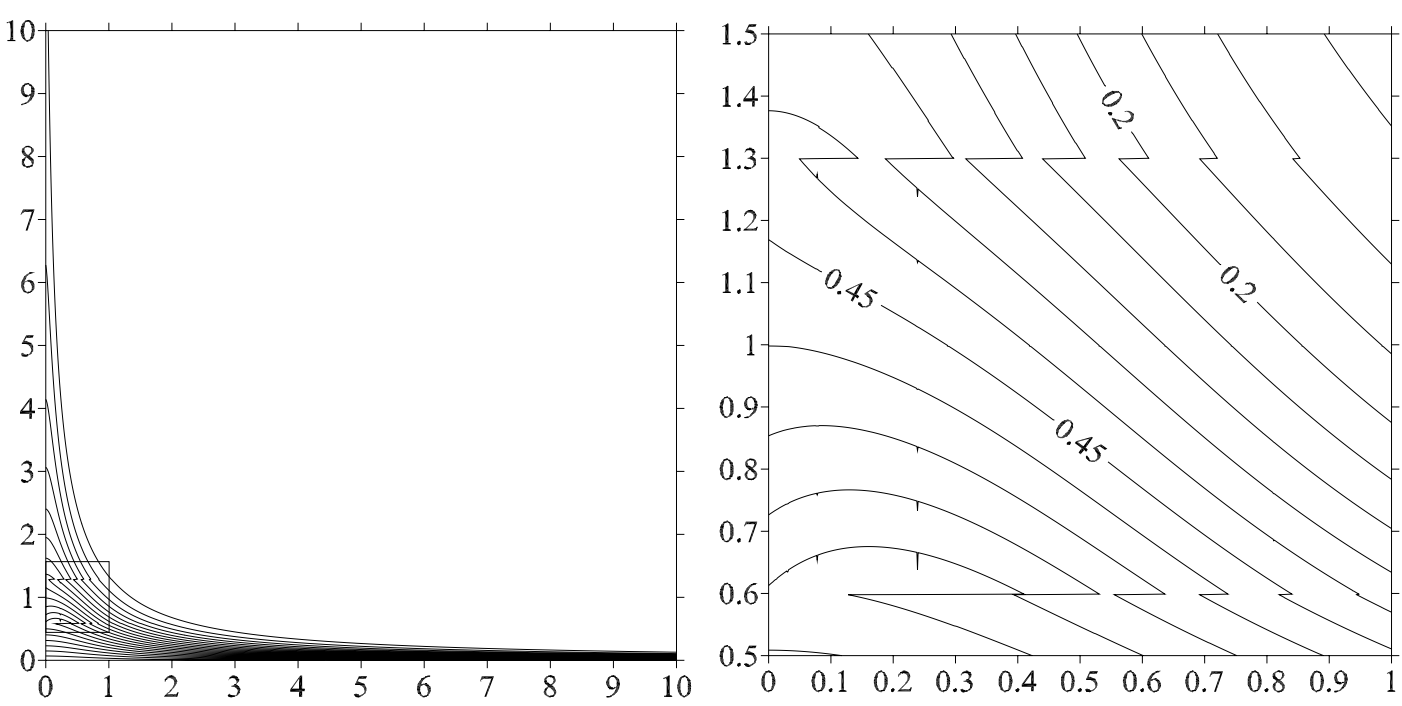

Figura 3.5.6 - Curvas de isovalores obtidas da avaliação de Barnes e Strack (2003) da integral de Rosser. À direita, detalhe da descontinuidade observada na transição em torno da ordenada igual a um.

Observa-se, ainda, que uma vez que a avaliação de Barnes e Strack (2003) não seja válida para $\sqrt{\frac{\eta^{2}}{4 \alpha t}} \frac{\xi-\xi_{j}}{|\eta|}<1$ na faixa de ordenadas $\frac{|\eta|}{\xi-\xi_{j}} \approx 1$, a avaliação de Litkouhi e Becker (1982, apud Barnes e Strack, 2003) permanece válida em todo o domínio e pode permanecer sendo utilizada em $\frac{|\eta|}{\xi-\xi_{j}} \approx 1$ quando a abscissa $\sqrt{\frac{\eta^{2}}{4 \alpha t}} \frac{\xi-\xi_{j}}{|\eta|}$ for menor que 1,0 . Por outro lado, embora seja necessária uma elevada quantidade de termos para a convergência das séries utilizadas neste caso, introduz oscilações na avaliação, consideradas toleráveis no escopo deste trabalho, à medida que o valor da ordenada $\frac{|\eta|}{\xi-\xi_{j}}$ se aproxima de 1,0 (ver Figura 3.5.7). 

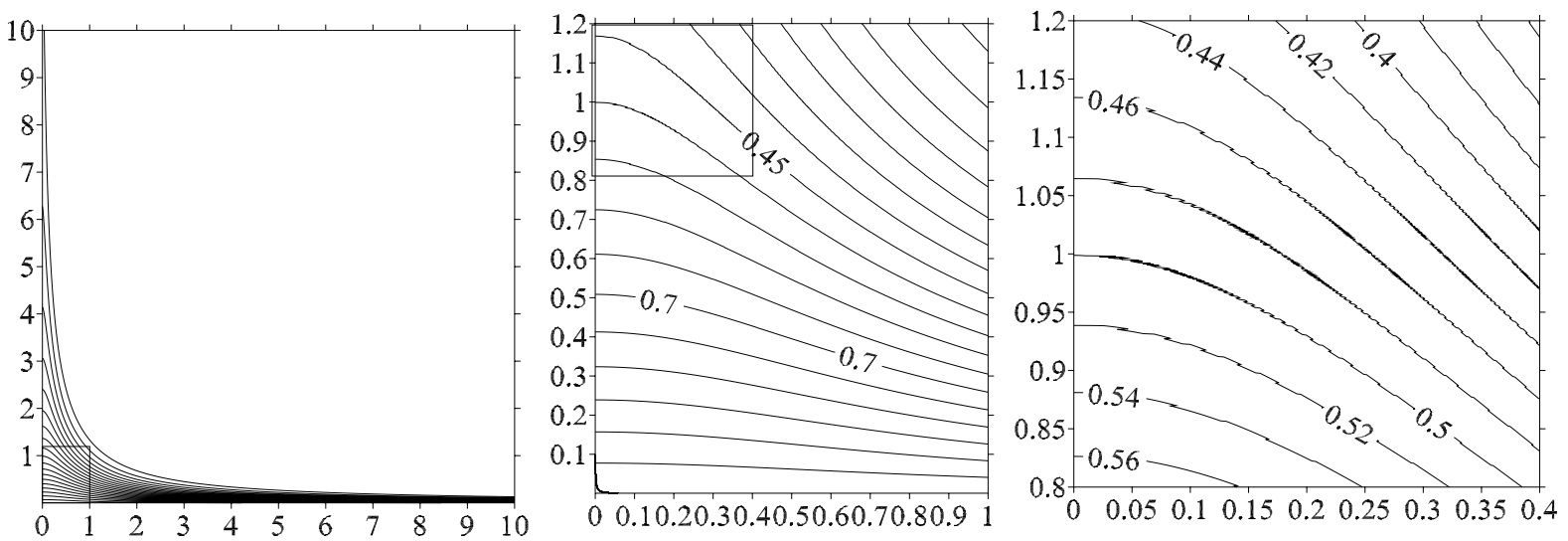

Figura 3.5.7 - Curvas de isovalores para a integral de Rosser. Avaliação obtida em torno de $y=1$ sem descontinuidade

\subsection{Exemplo de Aplicação}

A necessidade do uso de sobreposição de efeitos no tempo e os problemas encontrados na avaliação da integral de Rosser têm restringido o emprego da formulação de Zaadnoordijk (1988) em problemas práticos. Por outro lado, a formulação apresenta-se bastante útil para o estudo de pequenos problemas de escoamento em planos infinitos fornecendo excelentes resultados teóricos.

Zaadnoordijk e Strack (1993) apresentam um estudo de validação e um de aplicação da formulação proposta em Zaadnoordijk (1988). No estudo de validação, Zaadnoordijk e Strack (1993) obtiveram boa concordância com a solução analítica unidimensional da elevação súbita do potencial na fronteira do semi-plano $x \geq 0$ utilizando-se elementos linesink de grau zero. O estudo apresenta, também, um sistema hipotético de irrigação intermitente modelado com elementos analíticos transientes. Os exemplos apresentados em Zaadnoordijk e Strack (1993) são novamente utilizados neste trabalho com o objetivo de avaliar os resultados obtidos da modelagem com elementos de segunda ordem e grau zero (seção 3.3). Os exemplos são simulados com o programa $\operatorname{Tim}^{\mathrm{SL}}$ modificado, conforme apresentado anteriormente nesta seção. Os arquivos de entrada das simulações de ambos exemplos encontram-se disponíveis no CD em anexo.

O primeiro exemplo trata de um escoamento unidimensional gerado a partir de uma mudança súbita de potencial na fronteira. O domínio é semi-infinito e fronteira é definida no eixo $x=0$. Até o instante $t=0$ o potencial vale $\Phi_{0}=0$. Nesse instante, o potencial é subitamente elevado para $\Phi_{1}=1$. O escoamento devido à tal variação de potencial é descrito 
pela Equação do Calor (Eq. 3.3.1) e resolvida pela função Eq. 3.6.1, encontrada, por exemplo, em Carslaw e Jaeger (1959).

$$
\Phi=\Phi_{0}+\left(\Phi_{1}-\Phi_{0}\right) \operatorname{erfc}\left(\frac{x}{\sqrt{4 \alpha t}}\right)
$$

onde $\alpha$ é o coeficiente de difusividade do meio.

Zaadnoordijk e Strack (1993) constroem o modelo de elementos analíticos utilizando-se elementos linesink ao longo do eixo $x=0$ desde $y=-20$ até $y=20$. Para manter a condição de $\Phi=\Phi_{1}$ em $x=0$ é preciso calcular os parâmetros de esforços sobre cada linesink necessários para fornecer o potencial desejado. Os parâmetros são calculados utilizando-se intervalos de tempo $2 \frac{e^{2}{ }^{2}}{\alpha}$ e $\frac{1}{2} \frac{\stackrel{e l}{2}^{2}}{\alpha}\left(L_{e l}\right.$ representa o comprimento dos elementos utilizados $)$ conforme definidos arbitrariamente em Zaadnoordijk e Strack (1993). Na Figura 3.6.1 os resultados obtidos por Zaadnoordijk e Strack (1993) e obtidos com os elementos linesink deduzidos na seção 3.3.2 são confrontados com a solução exata no ponto de coordenadas $x=0, y=0$.

Em decorrência da escolha do ponto $(y=0)$, a ordem da distribuição espacial dos esforços sobre o elemento não faz diferença. Observe-se que ambas aproximações de carga piezométrica na fronteira, primeira ordem (Zaadnoordijk e Strack,1993) e segunda ordem (seção 3.3.2), são quase coincidentes (Figura 3.6.1, gráfico à esquerda). As diferenças encontradas são, provavelmente, devido ao diferente número de elementos utilizados em cada trabalho. As características da aproximação temporal de grau zero dos esforços tornam-se mais evidentes na resposta dada para a vazão na fronteira (Figura 3.6.1, gráfico à direita). A curva de recessão é aproximada por degraus, da mesma forma que em Zaadnoordijk e Strack (1993). Os valores obtidos de elementos analíticos não coincidem com a solução exata, mas os erros são menores para menores intervalos de tempo com que são calculados os parâmetros. 


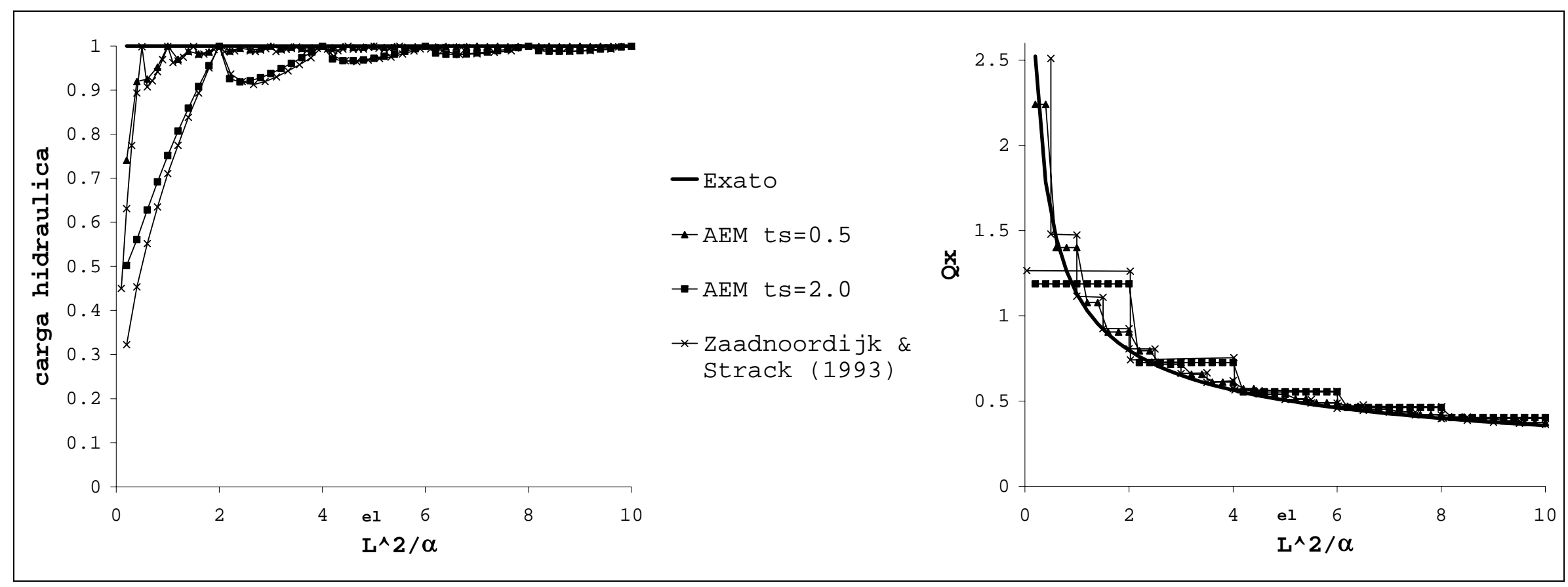

Figura 3.6.1 - Evoluções da carga piezométrica e da vazão obtidas de elementos analíticos em comparação com solução exata 
No segundo exemplo, é definido um sistema de irrigação e seus dados hidrogeológicos. Três áreas de cultivo irrigado são implantadas em uma região compreendida por dois braços de rio com cota de elevação do nível de água em $10 \mathrm{~m}$. O aqüífero sobre o qual encontra-se a área de plantio é confinado e possui uma cota média de elevação do fundo igual a $0,0 m$, condutividade hidráulica de $40 \mathrm{~m} /$ dia, espessura de 7,5m e coeficiente de armazenamento de $30 \%$, de maneira que a difusividade do aqǘfero tem o valor de $1000 \mathrm{~m}^{2} / \mathrm{dia}$. Os valores de recarga são descritos semestralmente e alternam entre $0,0 \mathrm{~mm} /$ dia e 1,0mm/dia. O sistema é abastecido por meio de cinco poços localizados na mesma região e tem o início da operação no quarto semestre considerado na simulação, correspondente ao segundo semestre seco. As condições de operação (canto superior esquerdo da Figura 3.6.2a) e o esboço do sistema foram retirados de Zaadnoordijk e Strack (1993) e são apresentados na Figura 3.6.2a.

A condição inicial da simulação é definida pelo modelo estacionário do escoamento anterior à implantação do sistema utilizando-se o valor médio das recargas semestrais. Essa parte da modelagem é feita com elementos estacionários. São utilizados, um areasink com quatrocentos e oitenta nós, representado pelo retângulo maior na Figura 3.6.2b e quinze linesinks. Semelhantemente, o escoamento não-permanente devido às variações de recarga até o início da operação do sistema é modelado utilizando a mesma quantidade de elementos, porém, agora, transientes. A partir do início da operação, são adicionados elementos analíticos ao modelo para a representação do bombeamento dos poços (círculos pequenos na Figura 3.6.2b) e da infiltração devido à irrigação das áreas de plantio representados por areasinks construídos com nós a cada vinte e dois metros (quadriláteros pequenos na Figura 3.6.2b).

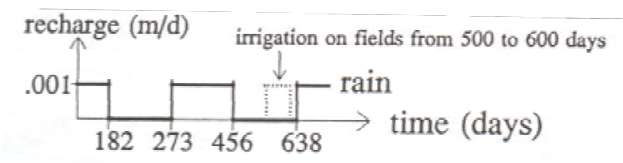

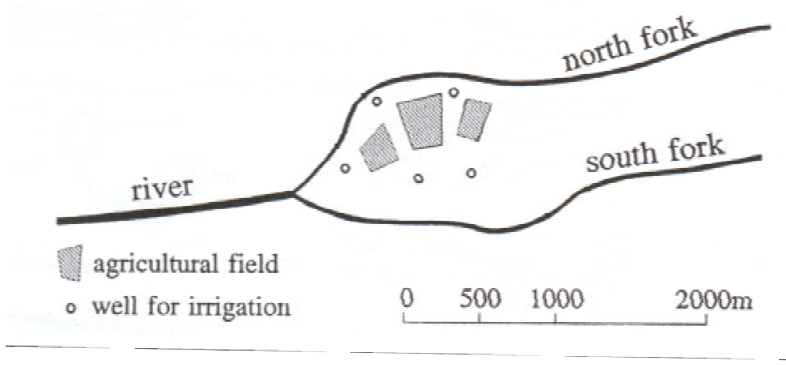

(a)

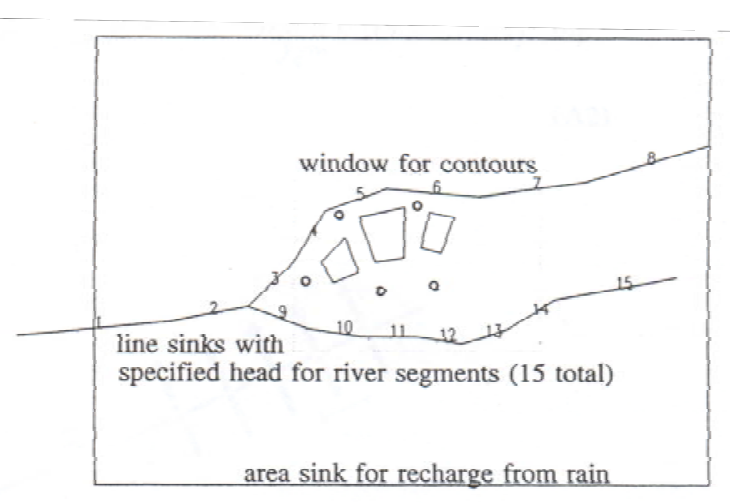

(b)

Figura 3.6.2 - Esboços para a simulação do sistema hipotético. (a) Mapa hipotético e operação do sistema. (b) Disposição de elementos analíticos para simulação do sistema (Retirado de Zaadnoordijk e Strack, 1993) 
Elementos areasink e poços representam fenômenos (efeitos) caracterizados por parâmetros previamente conhecidos. Elementos areasink devidos à precipitação possuem

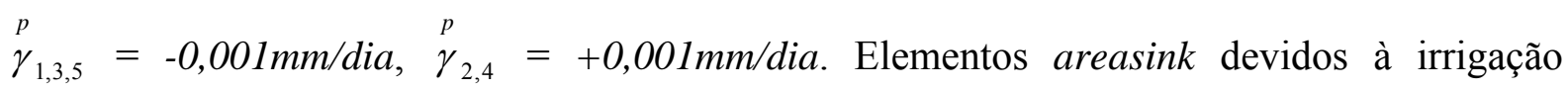
possuem $\stackrel{i r}{\gamma_{1}}=-0,001 \mathrm{~mm} /$ dia, ${ }_{2}^{i r} \gamma_{2}=+0,001 \mathrm{~mm} /$ dia. A partir da vazão necessária para irrigar as três áreas, os elementos poços devem possuir, cada, $\stackrel{i r}{Q_{1}}=+30,89 \mathrm{~m}^{3} / \mathrm{dia}, \stackrel{i r}{Q_{2}}=-30,89 \mathrm{~m}^{3} / \mathrm{dia}$. Os índices sobrescritos nos parâmetros acima indicam a ordem com que eles serão utilizados no modelo. Assim, os valores opostos de cada parâmetro significam que estão sendo introduzidos efeitos contrários, via-de-regra, para eliminar um efeito anterior.

Os elementos linesink, entretanto, não possuem os esforços pré-determinados. Assim, o valor do potencial equivalente à carga piezométrica observada nos rios é especificado em pontos de controle ao longo das linhas. Semelhantemente ao primeiro exemplo, é estabelecida uma marcha de cálculo dos parâmetros, de modo, mais uma vez, a impor o potencial ao contorno (os rios) a cada intervalo de 25dias, o mesmo definido no trabalho anterior (ver Eq. 3.4.31). Não foram fornecidas a posição do ponto de referência nem a carga piezométrica de referência. Assim, o modelo construído para este exemplo, considera o ponto de referência situado $1000 \mathrm{~m}$ à esquerda e $400 \mathrm{~m}$ abaixo do vértice inferior esquerdo do grande areasink representado na Figura 3.6.2b). O valor da carga piezométrica naquele ponto foi considerado igual a $10 \mathrm{~m}$.

Os resultados obtidos do modelo construído para este exemplo são apresentados a seguir. Muito embora os resultados de Zaadnoordijk e Strack (1993) não tenham podido ser reproduzidos quantitativamente por falta de dados disponíveis, são evidenciadas melhorias na forma das curvas potenciométricas em relação ao trabalho anterior. Essas melhorias são atribuídas à elevação da ordem dos elementos (3.3.2) e da implementação conjunta das avaliações disponíveis para a integral de Rosser (3.5.5). Na Figura 3.6.3 apresenta-se as linhas equipotenciais obtidas do modelo acima para o tempo $t=730$ dias em uma janela que abrange todos os elementos utilizados. Embora não tenham sido disponibilizados resultados dessa abrangência em Zaadnoordijk e Strack (1993), para que se pudesse produzir comparações, observa-se suavidade na superfície potenciométrica aqui obtida. 


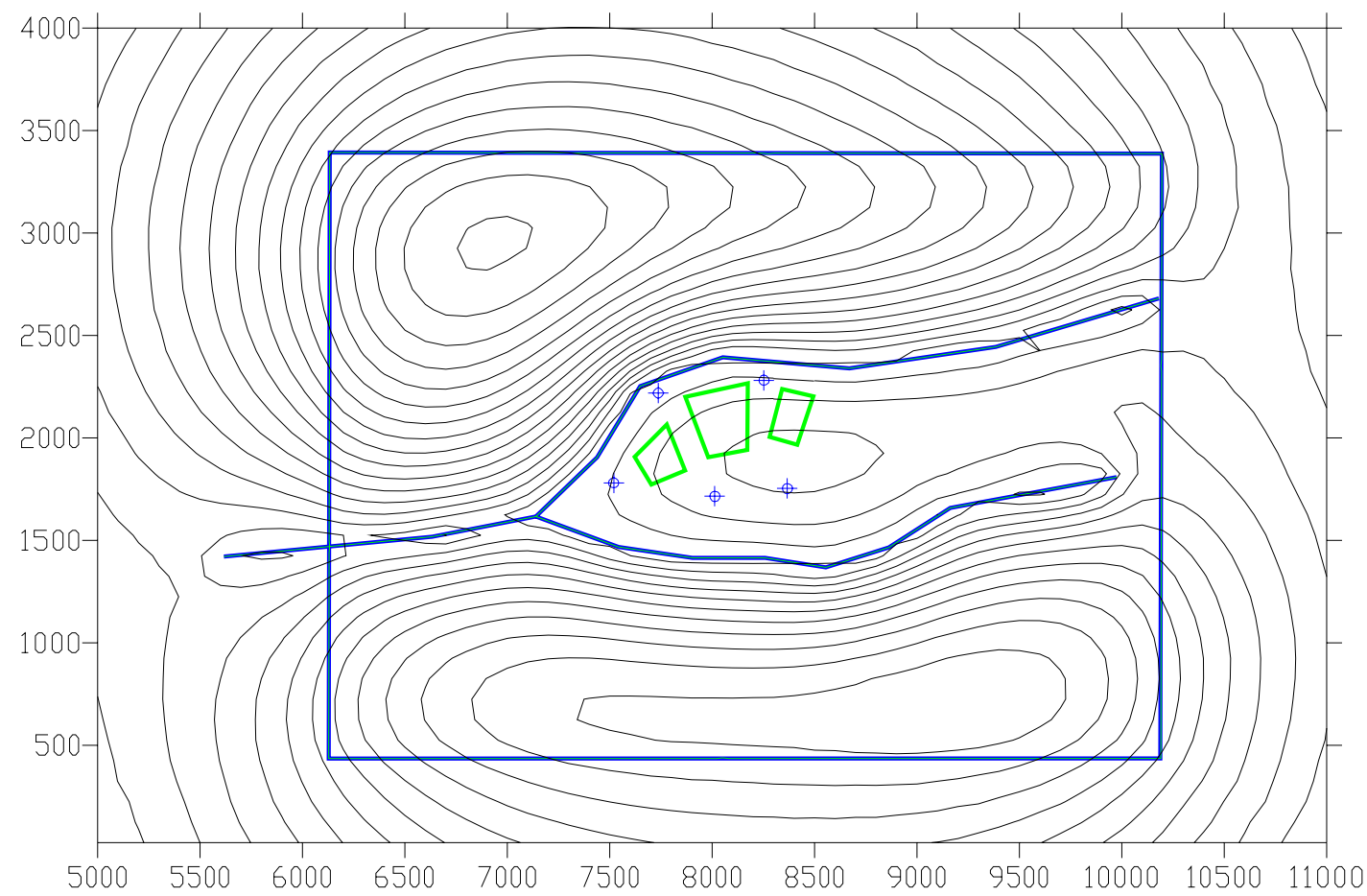

Figura 3.6.3 - Distribuição de cargas piezométricas sobre todos os elementos do modelo no instante $t=730$ dias

Os resultados podem ser comparados com o trabalho anterior na Figura 3.6.4. São apresentadas oito janelas assinaladas com o instante de tempo com os quais se relacionam. Novamente observa-se (linhas pontilhadas na Figura 3.6.4) a suavidade da superfície aqui obtida em cada instante representado. Problemas relacionados à avaliação da integral de Rosser são evidenciados nos resultados apresentados por Zaadnoordijk e Strack (1993) em locais próximos aos braços de rio e em tempos finais de períodos secos, $t=273$ dias e $t=$ 637dias (ver linhas contínuas na Figura 3.6.4) 


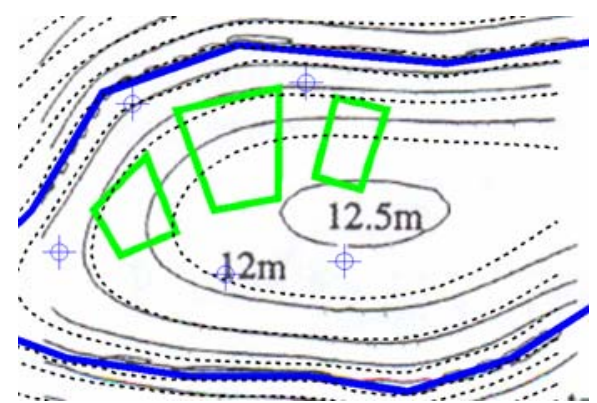

91 dias

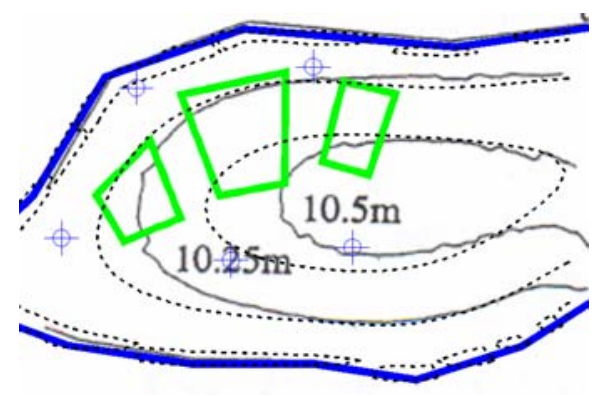

273 dias

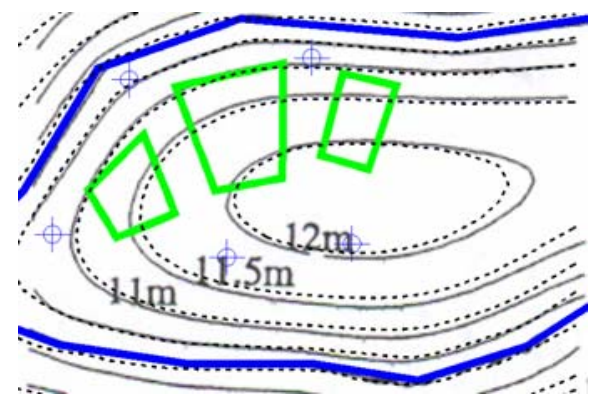

455 dias

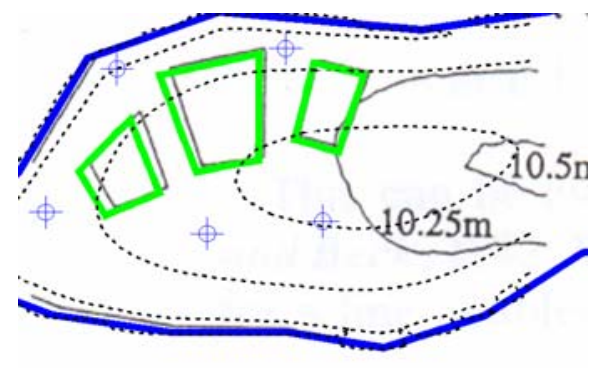

637 dias

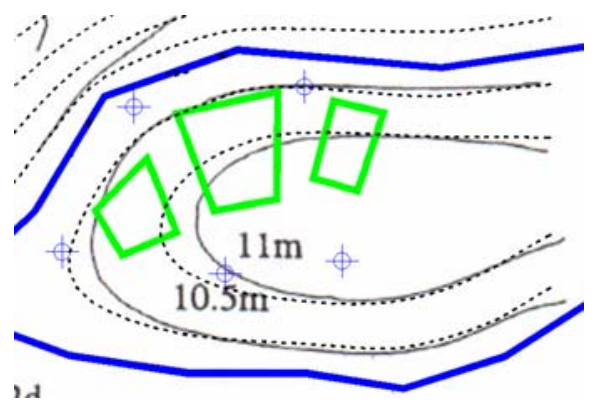

182 dias

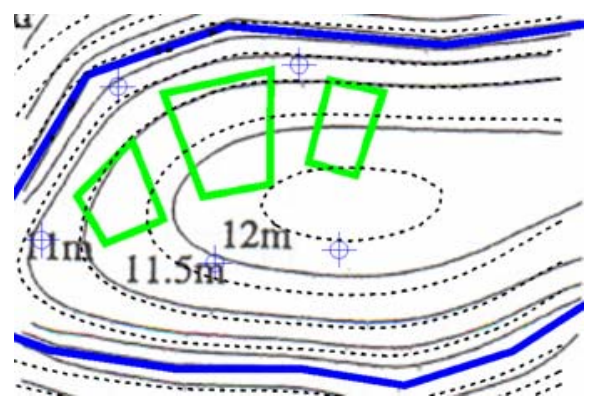

365 dias

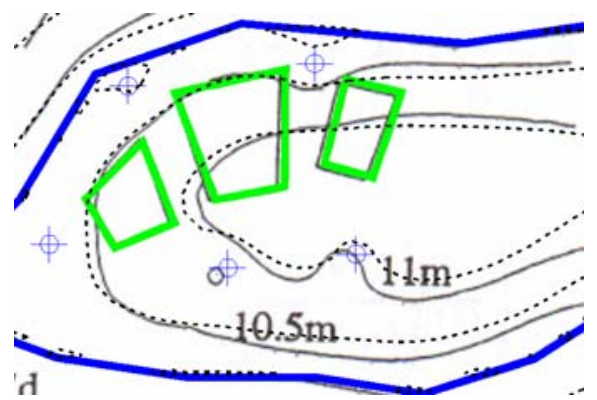

546 dias

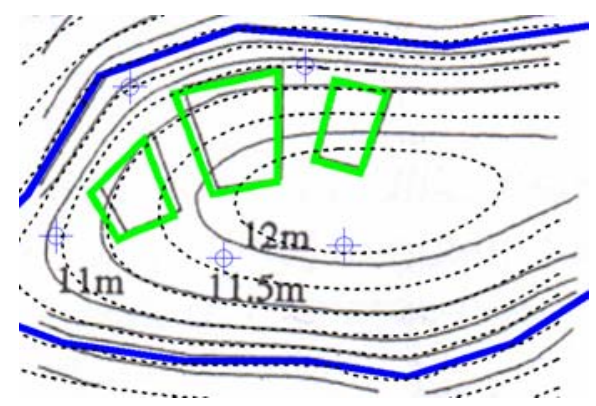

730 dias

Figura 3.6.4 - Distribuições de cargas piezométricas obtidas em diversos instantes da simulação (linhas pontilhadas) em comparação com aquelas obtidas de Zaadnoordijk e Strack (1993) (linhas contínuas) 


\section{Capítulo 4}

\section{ALTERNATIVAS AO USO DE ELEMENTOS EM MODELOS DE AQÜÍFEROS LONGOS}

As configurações geométricas dos elementos apresentados no capítulo 3 são convenientes para a representação das estruturas comumente encontradas em aqüíferos. No entanto a disposição de elementos analíticos ao longo de fronteiras externas de muito extensos pode acarretar algumas dúvidas: 1) quanto à extensão a ser considerada na borda e 2) quanto ao dimensionamento dos elementos ali posicionados. Neste capítulo, serão discutidas e utilizadas técnicas alternativas ao emprego de elementos analíticos em representações de contornos externos. Observa-se no decorrer da discussão que técnicas como o Método de Imagens e as transformações de Schwarz-Christoffel permitem dispensar o uso de elementos em determinadas configurações geométricas dessas fronteiras e portanto, as possíveis dúvidas mencionadas.

Como visto na seção 2.4.2, o Método de Imagens é baseado no princípio da sobreposição de efeitos e visa à especificação de fronteiras retilíneas a partir da simetria entre funções definidas em subdomínos. As transformações de Schwarz-Christoffel, por sua vez, são válidas para qualquer polígono de $n$ lados, embora suas soluções não sejam obtidas facilmente em domínios irregulares. Não obstante as geometrias observadas em aqüíferos serem irregulares, é possível fazer uso de idealizações em fronteiras externas. Neste trabalho, as simplificações geométricas de domínio são baseadas na seguinte premissa: uma vez definida uma região de interesse no modelo menor que o domínio do escoamento, a complexidade da geometria de fronteiras externas exercerá tanto menor influência no escoamento quanto mais distante estiverem da região de interesse. Quando a representação de Aqüíferos Longos por apenas duas linhas retas é possível, as transformações de SchwarzChristoffel são obtidas e o Método de Imagens é então empregado na fronteira de um semiplano (ver Figura 2.4.1). Nesta seção, Método de Imagens e transformações de SchwarzChristoffel são discutidos em termos de sua aplicabilidade em problemas de escoamentos subterrâneos. 


\subsection{Escopo do Método de Imagens}

O Método de Imagens é uma técnica clássica, baseada na sobreposição de efeitos, amplamente difundida em aplicações da Equação de Laplace (e.g. Águas Subterrâneas, Eletrostática e Eletromagnetismo). Maxwell (1873 apud Muskat 1937) ${ }^{12}$ atribui a origem do método a William Thomson em 1848. Com freqüência o Método de Imagens é empregado com o objetivo de obter expressões para escoamentos bi e tridimensionais sujeitos a fronteiras externas. Strack (1989), por exemplo, apresenta diversos modelos de escoamentos bidimensionais estacionários e transientes para poços mediante a aplicação do Método de Imagens. Em domínios tridimensionais, diversos autores (e.g. Muskat, 1937; Luther e Haitjema, 2000 e Steward, 2001) utilizam o método na abordagem de escoamentos produzidos por poços de perfuração parcial.

O Método de Imagens é definido, segundo Keller (1953), como um método para a construção de funções de Green em regiões delimitadas por planos. O objetivo do método é especificar condições de contorno mediante a sobreposição de funções de Green primitivas (Eq. 4.1.1). A partir dessa definição, o autor determinou regiões, equações diferenciais e condições de contorno para as quais o método é válido.

$$
\mathrm{G}\left(r, r^{\prime}\right)=\sum_{r^{\prime \prime} \in S(r)} \mathrm{G}^{*}\left(r^{\prime \prime}, r^{\prime}\right)
$$

A equação Eq. 4.1.1 descreve a função de Green para dois pontos no espaço, $r$ e sua imagem $r$ '. A função $G^{*}$ por sua vez é definida para "imagens de imagens" ( $r$ ") e seus pontos originários $\left(r^{\prime}\right)$. O enunciado da proposição de Keller (1953) pode ser apresentado da seguinte forma, considerando-se $D$ a região admissível para a qual o Método de Imagem é aplicável e a reflexão em um lado $i$ dessa região $(D)$ representada por $R_{i}$. Considere-se ainda que $S(r)$ é o conjunto de imagens de um ponto $r$ no domínio $D$ definido pelo conjunto de pontos obtidos pela aplicação de todas as reflexões do ponto $r$ (Figura 4.1.1). Se a região $D$ é admissível, o Método de Imagens tem o objetivo de obter um conjunto de imagens $S(r)$ de forma que este possua apenas um ponto correspondente para cada ponto da região $D$. Isso implica que o conjunto gerado pelas reflexões $R_{i}$ é discreto e que a região $D$ é o domínio fundamental desse grupo.

\footnotetext{
${ }^{12}$ Maxwell, J.C. (1873) A Treatise on Electricity and Magnetism. Clarendon Press, Oxford
} 


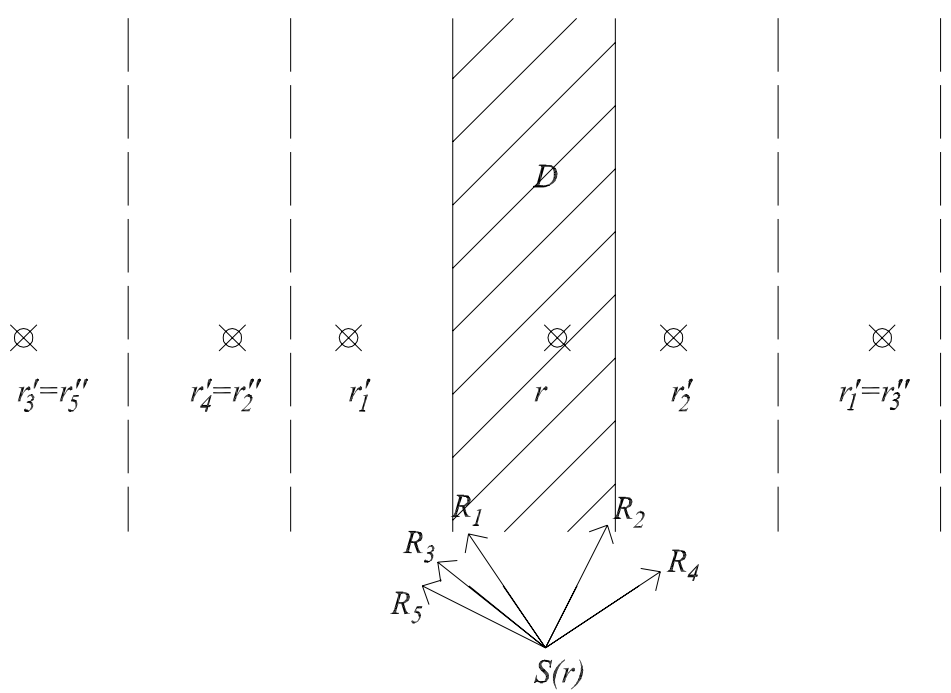

Figura 4.1.1 - Esboço esquemático dos termos definidos para o Método de Imagens

Para fronteiras não-paralelas, o ângulo deve ser uma divisão na forma $2 \pi / p$, onde $p$ é um inteiro. No entanto deve-se notar que se $p$ é ímpar, o conjunto $S(r)$ produz pelo menos dois pontos sobre a própria região $D$, deformando-se os pontos do domínio fundamental (Figura 4.1.2).

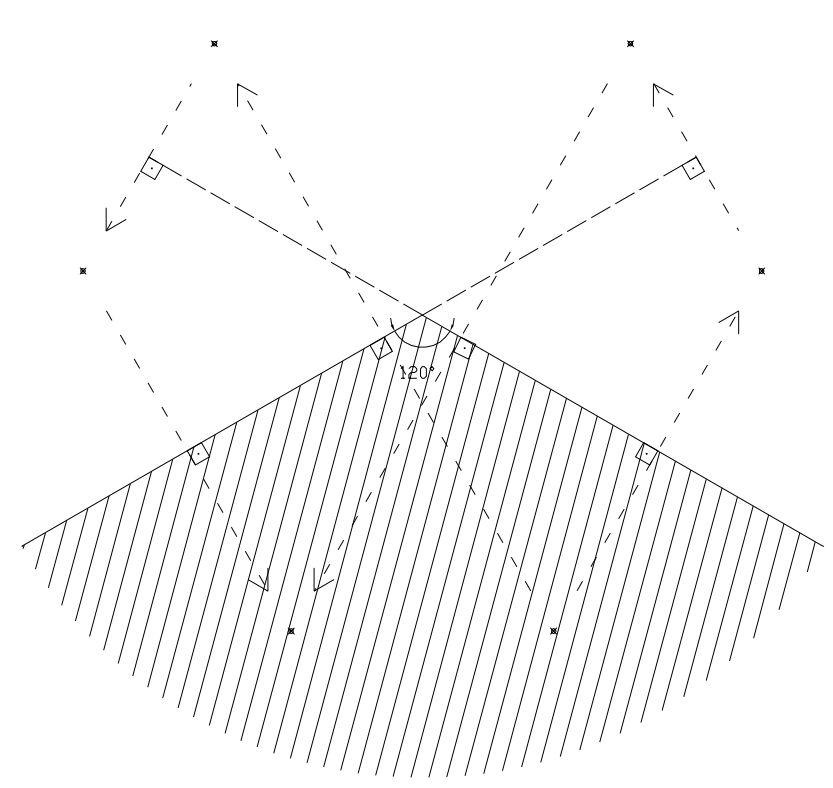

Figura 4.1.2 - Deslocamento não desejado de pontos do domínio fundamental

Portanto, apenas os domínios formados por planos unidos por ângulos da forma $\pi / p$ são constituídas regiões admissíveis para a aplicação do Método de Imagens. Keller (1953) 
concluiu que as geometrias das regiões admissíveis ao Método de Imagens para duas e três dimensões são encontradas para uma quantidade finita e determinada de planos. Assim, o número de imagens obtidas em torno desses contornos é igual à quantidade de setores circulares resultantes da abertura entre as linhas ou planos (Eq. 4.1.2). Em casos de geometrias fechadas (e.g. triângulos, retângulos, prismas e poliedros) é necessário um número infinito de imagens. No plano, a simetria é obtida a partir de um número finito de imagens somente para contornos formados por uma única reta ou por duas semi-retas concorrentes. Retas paralelas exigem o uso de infinitas reflexões.

$$
n=2 \pi / \alpha-1, \alpha=\pi / p
$$

Uma função de Green $\left(\mathrm{G}^{*}\right)$ sujeita a contornos do primeiro ou do segundo tipo deve ser especificada pelo Método de Imagens utilizando-se propriedades de funções pares e ímpares (ver seção 3.4.3). Assim, quando é imposto ao contorno que a função seja zero $(G=0)$ é especificada a simetria ímpar. Quando sua derivada em relação à normal da fronteira desejada é especificada com valor nulo $(\partial \mathrm{G} / \partial \eta=0)$ é verificada a simetria par. Observa-se nas expressões Eq. 2.4.1 e Eq. 2.4.2 que as componentes de suas derivadas relacionam-se por intermédio ora da igualdade, ora do sinal oposto. Da mesma forma, o somatório Eq. 4.1.1 especifica condições do primeiro ou do segundo tipo multiplicando-se a função imagem por menos um (-1) quando necessário, de modo a obter a simetria desejada. Deve-se observar ainda que, se a especificação das condições não é a mesma em todas as linhas ou planos fronteiriços, a verificação do ângulo de simetria entre os contornos é analisada de forma que o ângulo de simetria $(\alpha)$ é encontrado entre aquelas fronteiras de igual especificação.

O Método de Imagens pode ser aplicado em qualquer equação linear. No entanto, sua aplicação em diversas fronteiras simultaneamente depende da simetria do comportamento da equação na direção de cada fronteira. No caso de duas variáveis, uma equação diferencial homogênea pode ser representada de forma geral por:

$$
\omega_{1} \partial^{2} \phi / \partial x^{2}+\omega_{2} \partial^{2} \phi / \partial x \partial y+\omega_{3} \partial^{2} \phi / \partial y^{2}+\omega_{4} \partial \phi / \partial x+\omega_{5} \partial \phi / \partial y+\omega_{6} \phi=0 \quad \text { Eq. 4.1.3 }
$$

Tomando $x^{\prime}, y^{\prime}$ como as imagens de $x, y$ em torno de uma linha que faz um ângulo $\theta$ com o eixo $x$, Keller (1953) define que o Método de Imagens é aplicável quando a forma da equação (Eq. 4.1.3) é mantida mesmo após a transformação das variáveis. Reescrevendo a 
equação Eq. 4.1.3 para os eixos rotacionados $\left(x^{\prime}, y^{\prime}\right)$, Keller (1953) demonstra, por exemplo, que para valores de $\omega_{6}$ e $\omega_{2}$ nulos e $\omega_{1}=\omega_{3}$, a equação Eq. 4.1 .3 não depende do valor de $\theta$. Isso significa, por exemplo, que a Equação de Laplace ( $a=c$ na Eq. 4.1.3), por exemplo, não sofre variação para qualquer valor de $\theta$. Na Tabela 4.1.1 são reunidos os valores de coeficientes de Eq. 4.1.3 para os quais admite-se a utilização do Método de Imagens (Keller, 1953).

Tabela 4.1.1 - Valores de $\theta$ admissíveis para o Método de Imagens

\begin{tabular}{l|l|l}
\hline$\omega_{6}=0$ & $\omega_{2}=0$ AND $\omega_{1}-\omega_{3}=0$ & qualquer $\theta$ \\
\hline$\omega_{6}=0$ & $\omega_{5} \neq 0$ AND $\omega_{1}+\omega_{3}=0$ & $-\frac{\omega_{4}}{\omega_{5}}=\frac{\operatorname{sen} 2 \theta}{1+\cos 2 \theta}$ \\
\hline$\omega_{6}=0$ & $\omega_{5}=0$ AND $\omega_{1}+\omega_{3}=0$ & $\frac{2 \omega_{1}}{\omega_{2}}=\tan 2 \theta$, para $\theta$ e $\theta+\pi / 2$ \\
\hline$\omega_{6} \neq 0$ & $\omega_{5}=\omega_{4}=\omega_{2}=0$ AND $\omega_{1}=\omega_{3}$ & qualquer $\theta$ \\
\hline$\omega_{6} \neq 0$ & $\omega_{5}=0$ XOR $\omega_{4}=0$ AND $\omega_{2}=0$ AND $\omega_{1}=\omega_{3}$ & $\frac{\omega_{5}}{\omega_{4}}=\frac{\operatorname{sen} 2 \theta}{1-\cos 2 \theta}$ \\
\hline$\omega_{6} \neq 0$ & $\omega_{5}=0$ XOR $\omega_{4}=0$ AND $\omega_{2}=0$ XOR $\omega_{1}=\omega_{3}$ & $\frac{\omega_{5}}{\omega_{4}}=\frac{\operatorname{sen} 2 \theta}{1-\cos 2 \theta}$ e $\frac{\omega_{2}}{\omega_{1}-\omega_{3}}=\tan 2 \theta$ \\
\hline$\omega_{6} \neq 0$ & $\omega_{5}=\omega_{4}=0$ AND $\omega_{2}=0$ XOR $\omega_{1}=\omega_{3}$ & $\frac{\omega_{2}}{\omega_{1}-\omega_{3}}=\tan 2 \theta$, para $\theta$ e $\theta+\pi / 2$ \\
\hline
\end{tabular}

\subsection{Transformações de Schwarz-Christoffel para Duas Fronteiras Retilíneas}

Como já mencionado, a transformação de Schwarz-Christoffel expressa a relação entre um contorno poligonal e um semi-plano, onde a condição de contorno é, então, imposta por meio do Método de Imagens. Embora esta técnica seja válida apenas para domínios planos, sua aplicação constitui-se tão ampla quanto à representação de geometrias planas por intermédio de polígonos. A abordagem de contornos poligonais mediante transformações de Schwarz-Christoffel abrange uma ampla literatura especializada e sua apresentação foge ao escopo deste trabalho. São discutidos aqui dois casos cujas soluções são dadas em Strack (1989): domínios encontrados em duas fronteiras paralelas e em duas fronteiras concorrentes.

Considere-se o caso de uma faixa formada por duas fronteiras paralelas onde são especificadas as mesmas condições de contorno. Na Figura 4.2.1a é ilustrado o domínio da aplicação. Os pontos 1 e 3 são arbitrados nas extremidades da faixa, ou seja, em $+\infty$ e $-\infty$ respectivamente. 


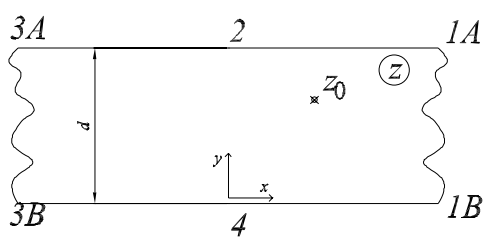

(a)

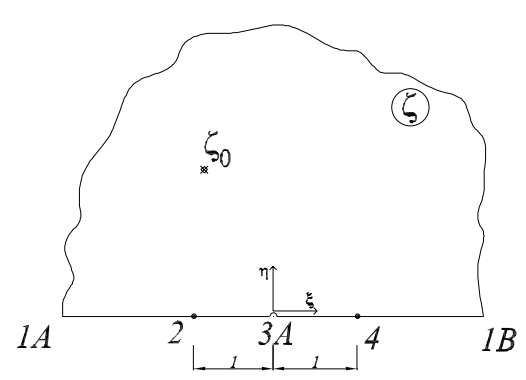

(b)

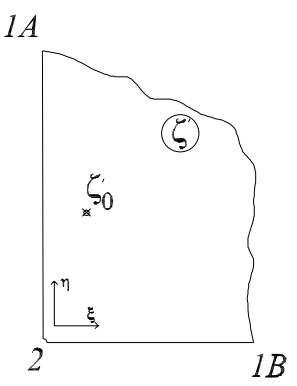

(c)

Figura 4.2.1 - Esboço para transformações em uma faixa infinita

O semi-plano $\zeta$, a partir do qual a faixa é mapeada, é especificado de forma que sua origem corresponda ao ponto 3 da faixa e os extremos do eixo correspondam ao ponto 1 (Figura 4.2.1b). Os termos $k_{v}$ e $\varphi$ da expressão geral da transformação (Eq. 2.4.12) são obtidos da seguinte forma: observando-se a equação Eq. 2.4.3 tem-se que $k_{1}$ e $k_{3}$ são avaliados fazendo:

$$
\arg \left(z_{v}-z_{v+1}\right)-\arg \left(z_{v}-z_{v-1}\right)=\pi k_{v}
$$

Tomando-se as medidas dos ângulos $\arg (\mathrm{z})$ no segmento $v=1$ e no segmento $v=3$, em sentido anti-horário, tem-se $k_{1} \pi=k_{3} \pi$ e conseqüentemente $k_{3}=k_{1}=1$. Ou seja, o argumento de $d z$ aumenta de $\pi$ comparando-se os segmentos $1 A-2$ e 4-1B e o mesmo para os segmentos 2-3A e 3B-4. O parâmetro $\varphi$ por sua vez, é obtido da equação Eq. 2.4.9 como $\varphi=\arg \frac{d z}{d \xi}=0$. Assim, a transformação assume a forma:

$$
Z=|A| \int \frac{d \zeta}{\zeta}+B=|A| \ln \zeta+B
$$

onde $B$ é uma constante complexa. Aplicando-se as condições do mapeamento nos seguintes contornos:

$$
\eta=0 \quad 0<\xi<\infty \quad \text { para } \quad y=0 \quad-\infty<x<\infty
$$




$$
\eta=0 \quad-\infty<\xi<0 \text { para } y=d \quad-\infty<x<\infty
$$

a expressão Eq. 4.2.2 produz, respectivamente:

$$
\begin{aligned}
& x=|A| \ln |\zeta|+B_{1} \\
& x+i d=|A| \ln |\zeta|+|A| i \pi+B_{1}+i B_{2}
\end{aligned}
$$

Ambas expressões estão definidas apenas no eixo $\xi$ onde as componentes imaginárias de $\zeta$ são nulas. Assim, aplicando-se $x=0$, na primeira expressão, tem-se $B_{1}=0$. Igualando-se as partes imaginárias na segunda expressão tem-se $|A|=d / \pi$, donde Eq. 4.2 .2 torna-se:

$$
z=\frac{d}{\pi} \ln \zeta
$$

e sua transformada inversa:

$$
\zeta=e^{\pi z / d}
$$

As expressões obtidas acima relacionam pontos de um semi-plano a pontos compreendidos entre duas retas paralelas. Assim, as condições físicas especificadas nos contornos da faixa devem ser iguais para que estas permaneçam constantes ao longo do eixo $\xi$. Em problemas onde são especificadas diferentes condições de escoamento, o mapeamento deve ser feito a partir de diferentes eixos para cada condição especificada. Considere-se o caso onde é imposta a condição do primeiro tipo sobre a linha entre os pontos $3 B$ e $1 B$ na Figura 4.2.1a e a condição do segundo tipo na linha entre $1 A$ e $3 A$. Tomando-se como ponto de partida o resultado obtido em (Eq. 4.2.5) observa-se que efetuando-se $\zeta^{1 / 2}$, define-se um novo semi-plano $\zeta$ simétrico em relação ao eixo $\xi=0$ (Figura 4.2.1c). Dessa forma, a condição observada sobre a linha 3B-1B será especificada sobre o eixo- $\xi$ ' e a condição dada em 1A-3A passa a ser especificada sobre o eixo $\eta^{\prime}$. Assim, pode-se dizer que o semi-plano $\zeta$ relaciona-se a $\zeta$ por meio de: 


$$
\zeta^{\prime}=\zeta^{\frac{1}{2}}
$$

Assim, o semi-plano $\zeta$ é mapeado no plano $z$ mediante:

$$
z=\frac{d}{\pi} \ln \left[\left(\zeta^{\prime}\right)^{2}\right]
$$

e sua transformada inversa dada por:

$$
\zeta^{\prime}=\sqrt{e^{\pi z / d}}
$$

Para o caso no qual o aqüífero é compreendido por duas fronteiras não paralelas, suas fronteiras podem ser representadas por meio de duas linhas retas concorrentes que formam um ângulo $\alpha$ qualquer entre si. Na Figura 4.2.2a os pontos 1A-2-1B representam a localização das fronteiras.

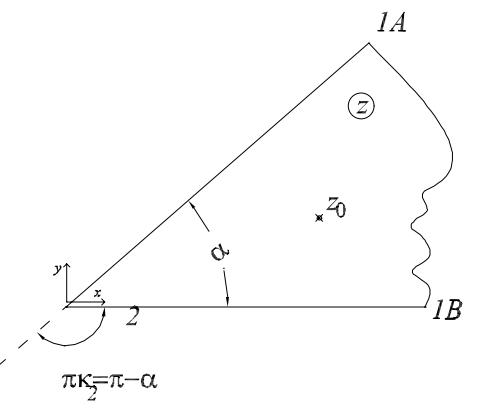

(a)

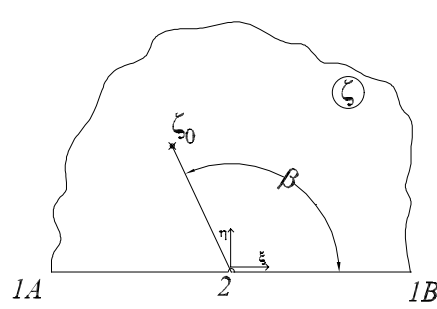

(b)

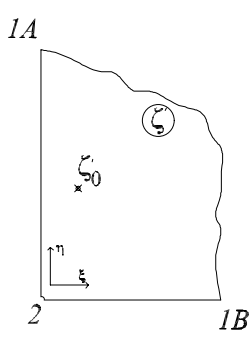

(c)

Figura 4.2.2 - Esboço para transformação em uma cunha infinita

Os pontos 1 e 2 no plano $z$ correspondem respectivamente a $z=\infty$ e à origem $z=0$. Obtendo-se os parâmetros $\varphi$ e $k_{v}$ num processo idêntico ao exemplo anterior, a relação entre ambos os planos obtida a partir da função Eq. 2.4.12 é:

$$
z=|A| \int \zeta^{-(1-\alpha / \pi)} d \zeta+B=\frac{\pi}{\alpha}|A| \zeta^{\alpha / \pi}+B
$$


De maneira que o ponto 2 faça correspondência a $\zeta=0$ (Figura 4.2.2b), obtém-se $B=0$, assim adotando-se um ponto de referência qualquer em $z\left(z=z_{0}\right) \operatorname{com} \zeta_{0}=e^{i \beta}$ :

$$
z_{0}=\frac{\pi}{\alpha}|A| e^{i \beta(\alpha / \pi)}
$$

Assim, a transformação do semi-plano $\zeta$ para o plano z é dada por:

$$
z=\left|z_{0}\right| \zeta^{\alpha / \pi}
$$

Em problemas onde são observadas condições do primeiro tipo sobre a fronteira 2-1B e do segundo tipo para a fronteira 1A-2, o semi-plano $\zeta$ é mapeado por $\zeta=\zeta^{1 / 2}$. Dessa forma, as condições dadas em cada fronteira são mapeadas a partir de cada eixo $\xi^{\prime}$ e $\eta^{\prime}$ como na Figura 4.2.2c. O mapeamento do semi-plano $\zeta$ para o domínio físico é dado então por:

$$
Z=\left|z_{0}\right| \zeta^{2 \alpha / \pi}
$$

e sua transformada inversa:

$$
\zeta=\left(\frac{Z}{\left|z_{0}\right|}\right)^{\frac{\pi}{2 \alpha}}
$$

\subsection{Uso de Elementos Analíticos em Domínios Alongados}

Nesta seção é discutida a utilização de elementos em modelos de domínios alongados. Primeiramente, funções que representam o potencial devido à infiltração uniforme de água em todo o domínio são apresentadas. Em seguida, o Método de Imagens é aplicado a ambos elementos analíticos, estacionários e transientes, apresentados no capítulo 3. Por fím, são discutidas as transformações de Schwarz-Christoffel sobre elementos estacionários.

Elementos transientes, como visto na seção 3.6, produzem bons resultados, porém, possuem aplicação restrita. Aplicações de transformações ou operações adicionais sobre esses 
elementos aumentam suas restrições de uso. Dessa maneira, apenas poucas operações adicionais, como no Método de Imagens, são aplicadas.

\subsubsection{Recarga Direta Compreendida por Duas Fronteiras Paralelas}

Aqüíferos $\operatorname{costeiros}^{13}$ e aqüíferos aluvionares, em geral, apresentam-se ao longo de extensas faixas de sedimentos contendo água à pressão atmosférica constituindo-se em Aqüíferos Longos. Nesses aqüíferos, as recargas provenientes de chuvas são infiltradas diretamente sobre o lençol freático e drenadas através do aqüífero até exutórios superficiais. Sendo assim, define-se um modelo para o efeito da recarga direta considerando de maneira idealizada a disposição das fronteiras do aqüífero paralelas à área de interesse abordada no modelo.

Pode-se, então, especificar as condições de escoamento ao longo do exutório principal (rio ou oceano) impondo-se que o potencial do aqǘffero seja igual ao potencial encontrado na superfície do corpo hídrico (Eq. 4.3.1). Por sua vez, as condições encontradas na fronteira de montante são, em geral, dadas pela formação vizinha. Considerando aqüíferos sedimentares, a formação encontrada na base do aqüífero comumente aflora constituindo-se também em bordas da formação sedimentar. Assim, em casos onde a base é constituída de rocha impermeável, a fronteira oposta é especificada de maneira que através dela não haja escoamento (Eq. 4.3.2).

Considerando que a linha de descarga principal esteja disposta paralelamente ao afloramento da base, o problema tem condições de contorno especificadas da seguinte maneira:

$$
\begin{array}{ll}
\text { para } \eta=0, & \Phi=\Phi_{0} \\
\text { para } \eta=L, & \partial \Phi / \partial \eta=0
\end{array}
$$

O escoamento assim descrito é dado pela Equação de Poisson cujo valor da fonte $(N)$ é dado positivo $(N>0)$ para extração e negativo $(N<0)$ para o aporte de água. Dispondo-se o sistema de coordenadas alinhando o eixo- $\xi$ sobre a linha de costa, a Equação de Poisson torna-se função de apenas uma variável independente $(\eta)$. Assim, a solução obtida para a

\footnotetext{
${ }^{13}$ No escopo deste trabalho, não é discutido o escoamento na interface entre água doce e água salgada.
} 
Equação de Poisson (Eq. 2.1.7) escrita na forma unidimensional, impostas as condições Eq. 4.3.1 e Eq. 4.3.2 é dada por:

$$
\Phi(\eta)=\frac{N}{2} \eta^{2}-N L \eta+\Phi_{0}
$$

Observe que a expressão não é válida em um domínio infinito como as expressões dos elementos discutidos no capítulo 3 , mas sim apenas na faixa que define o domínio do aqüífero. Neste sentido, vale ressaltar que as imagens e transformações utilizadas não compreendem a expressão acima (Eq. 4.3.3). Antes, o objetivo de tais operações sobre elementos utilizados em fronteiras internas do domínio é conformar seu comportamento à presença de fronteiras exteriores ao seu domínio.

O efeito produzido pela ocorrência de recargas intermitentes, ou não-permanentes, é representado sobre o mesmo domínio considerando um instante inicial $\left(t_{0}\right)$ e a intensidade $(N)$ para cada evento. A equação governante para o escoamento não-permanente é dada por uma Equação do Calor (Eq. 3.3.1) na forma unidimensional. Mantidas as condições especificadas para ambas fronteiras do aqǘfero, a solução para o problema pode ser encontrada, por exemplo, em Carslaw e Jaeger (1959), da seguinte forma:

$$
\Phi(\eta, t)=-\frac{4 N L^{2}}{\pi^{3}} \sum_{n=1}^{\infty} \frac{1}{n^{3}}\left\{1-\exp \left[-\left(t-t_{0}\right)\left(\frac{\pi n a}{L}\right)^{2}\right]\right\} \operatorname{sen}\left[\eta\left(\frac{\pi n a}{L}\right)^{2}\right]
$$

onde a difusividade hidráulica do aqüífero está definida como $a^{2}=K H / S$.

A aplicação da equação Eq. 4.3.4 segue as mesmas observações feitas para a equação (Eq. 4.3.3) em relação aos modelos de elementos analíticos. Entretanto, é interessante ainda mencionar o fato de que ambas equações Eq. 4.3.3 e Eq. 4.3.4 exibem a mesma estrutura onde um parâmetro de esforço multiplica uma função de posição (ver Eq. 3.2.4 e Eq. 3.3.10).

\subsubsection{Aplicação do Método de Imagens sobre Elementos Analíticos}

A especificação de condições de contorno via imagens constitui uma alternativa vantajosa em relação à distribuição de elementos analíticos ao longo de fronteiras externas. A princípio podem ser enumeradas três vantagens. Inicialmente, a alternativa apresenta 
vantagens do ponto de vista da construção do modelo, extinguindo-se a necessidade de se posicionar elementos ao longo de fronteiras extensas. Em segundo lugar, encontra-se uma vantagem maior no fato do Método de Imagens ser uma especificação exata. A especificação de condições de contorno por meio de elementos implica na sobreposição de uma quantidade finita de elementos constituindo-se uma aproximação para as condições esperadas. Por fim, deve-se considerar que os efeitos produzidos nos extremos da cadeia de elementos porventura utilizada sobre essas fronteiras podem estar presentes na área de interesse do modelo. Uma vez que os contornos obtidos pelo Método de Imagens são infinitos o efeito de borda é eliminado.

De acordo com a formulação apresentada no capítulo 3, um elemento analítico deve ser escrito na forma $\lambda_{(j)} \Lambda_{(j)}(\mathbf{r})$ onde $j$ é o ordinal referente a cada parâmetro de esforço da expressão do elemento. Sendo cada elemento dado por diversas parcelas de uma função harmônica ou soluções da Equação do Calor, cada parcela $j$ é também uma função, e todas estarão sujeitas individualmente a reflexões.

Seja $\lambda=\lambda_{j}^{e l}$ o esforço do elemento no domínio fundamental e $\lambda^{\prime}=\lambda_{e j}^{e l}$ o esforço no domínio atrás do "espelho". Assim, o elemento produzido em torno da fronteira do domínio será obtido definindo-se um termo $\Theta$ que relaciona ambos esforços $\lambda$ e $\lambda^{\prime}$ da seguinte forma:

$$
\lambda^{\prime}=\lambda_{e j}^{e l}=\underset{e l}{\stackrel{e}{e l}} \lambda_{j}
$$

onde o índice el refere-se ao elemento originário da imagem construída. O índice $e$ refere-se ao "espelho" (e) por intermédio do qual é construída a imagem de el, podendo el referir-se também a uma imagem se estiver ocorrendo recursão de imagens.

De acordo com Keller (1953), para encontrar a relação $\Theta$ entre uma imagem e sua origem, é necessário verificar a necessidade de multiplicar a imagem por menos um (-1) para obter a simetria desejada. Assim, especifica-se a condição de contorno determinando-se a direção dos esforços na imagem em função dos esforços no elemento originário simetricamente em relação à fronteira considerada. Utilizando-se a simbologia definida em Eq. 4.3.5, a condição do primeiro tipo é especificada para cada elemento definindo a relação $\underset{e l}{\stackrel{e}{e}}$ da seguinte forma: 


$$
\underset{\text { poço }}{\Theta}=-1
$$

$$
\left(\begin{array}{c}
I \\
\underset{\text { dipolo }}{\Theta}
\end{array}\right)_{\xi}=-1 \mathrm{e}\left(\begin{array}{c}
I \\
\Theta \\
\text { dipolo }
\end{array}\right)_{\eta}=1
$$

$$
\stackrel{I}{\Theta}=-1
$$

$$
\underset{\text { linedoublet }}{\stackrel{I}{\Theta}}=1
$$

$$
\stackrel{I}{\Theta}=-1
$$

onde os subscritos $\xi$ e $\eta$ referem-se às componentes do esforço em relação ao eixo da fronteira $(\xi)$ e à sua normal $(\eta)$. A condição do segundo tipo, por sua vez, é especificada para cada elemento da seguinte forma:

$$
\stackrel{I I}{\Theta}=1
$$

$$
\left(\begin{array}{c}
I I \\
\underset{\text { dipolo }}{\Theta}
\end{array}\right)_{\xi}=1 \text { e }\left(\begin{array}{c}
I I \\
\Theta \\
\text { dipolo }
\end{array}\right)_{\eta}=-1
$$

$$
\underset{\text { linesink }}{\stackrel{I I}{\Theta}}=1
$$

$$
\underset{\text { linedoublet }}{\stackrel{\text { II }}{\Theta}}=-1
$$

$$
\underset{\text { areasink }}{\stackrel{I I}{\Theta}}=1
$$


O sistema de equações para o cálculo dos esforços do modelo (Eq. 3.4.1) é construído relacionando-se os esforços na própria linha da matriz $\mathbf{A}$ dos coeficientes escrita para cada ponto de controle (ver seção 3.4). Assim, o termo $j$ de uma linha na matriz dos coeficientes leva em conta a relação especificada para sua imagem, a fim de impor a condição desejada nas fronteiras externas. Assim, cada coeficiente $a_{j}$ é expresso como:

$$
a_{j}=\Lambda_{j}^{e l}+\sum_{k, e=1}^{n, 2} \underset{e l}{e} \Lambda_{k}^{e l}
$$

O índice el refere-se ao elemento cujo esforço ocupa a posição $j$ na matriz. A construção das imagens do el-ésimo elemento empregado no modelo é feita de acordo com a fronteira (e) para a qual é feita a reflexão. Note-se que as imagens geradas poderão gerar novas imagens dependendo do ângulo entre os "espelhos" (ver Eq. 4.1.2). Em casos onde o número de “espelhos” é maior que 1 (um), $k$ será o número de iterações necessárias para a construção de todas as imagens, dado por $n=(2 \pi / \alpha) / 2=\pi / \alpha$, onde $\alpha$ é o ângulo entre os "espelhos". As

condições do terceiro tipo e a condição onde são impostos Dirichlet e Neuman simultaneamente (e.g. heterogeneidades), não são analisadas neste trabalho. No entanto, diversos autores discutem aplicações para poços sujeitos à presença de formações vizinhas e, recentemente, Anderson (2000) e Bakker e Anderson (2004) apresentam uma abordagem para problemas de poços sujeitos a fronteiras do terceiro tipo (ver Apêndice C).

\subsubsection{Aplicação de Transformações de Schwarz-Christoffel sobre Elementos Analíticos}

A formulação de elementos analíticos unifilares utilizados no Método de Elementos Analíticos é feita, originalmente, sobre o espaço contínuo e infinito. Nesta seção a formulação dos elementos utilizados no Método é modificada a fim de se considerar a modelagem de domínio alongado. Semelhantemente à formulação original, os elementos são baseados na integração de Cauchy para soluções da Equação de Laplace em problemas de efeitos pontuais no semi-plano que mapeia o domínio da aplicação. Devido ao mapeamento a geometria dos elementos no domínio da aplicação é diferente da geometria do elemento no semi-plano de mapeamento (ver Figura 4.3.1). 

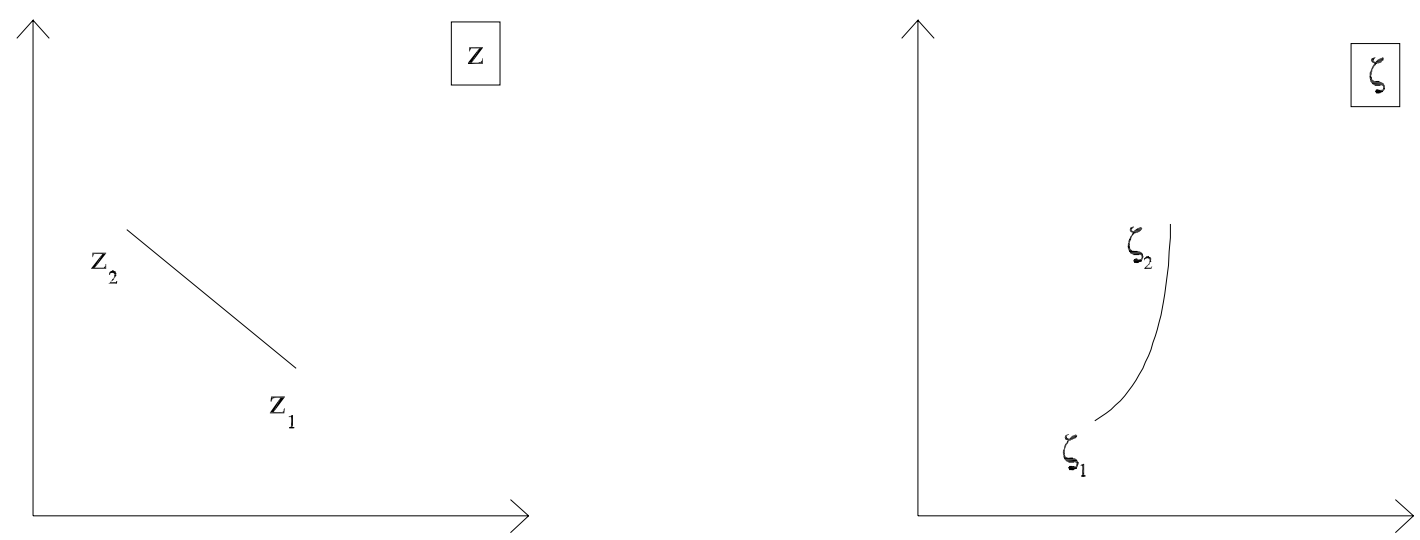

Figura 4.3.1 - Esboço do elemento no plano físico (esquerda) e no semi-plano de mapeamento (direita)

Devido a essa transformação, segmentos de reta no plano $z$ são mapeados por curvas espirais em z. Adimensionalizando-se o semi-plano $\zeta$ por meio da Eq. 4.3.17,

$$
Z=\frac{\zeta-\frac{1}{2}\left(\zeta_{1}+\zeta_{2}\right)}{\frac{1}{2}\left(\zeta_{2}-\zeta_{1}\right)}
$$

onde $\zeta_{1}$ e $\zeta_{2}$ são coordenadas complexas do elemento no semi-plano $\zeta$, tem-se uma nova configuração para o elemento conforme apresentado na Figura 4.3.2a.

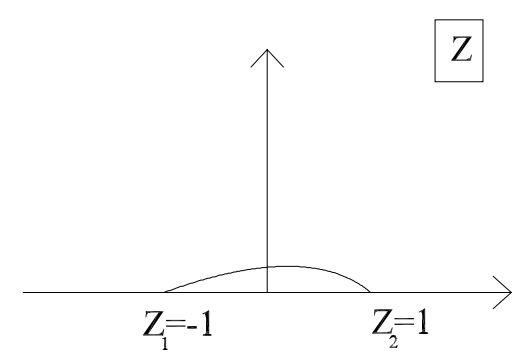

(a)

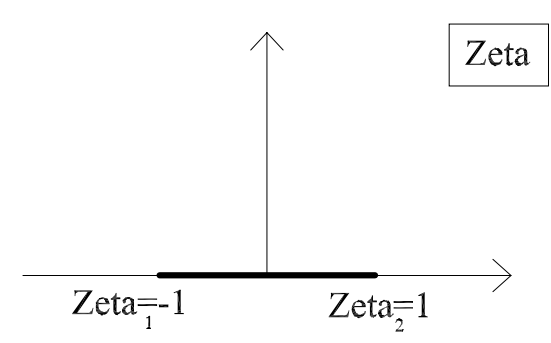

(b)

Figura 4.3.2 - Mapeamento do elemento (a) no plano Z e (b) no plano Zeta 
É possível mapear novamente o elemento para um novo plano (Figura 4.3.2b) que o representa sobre seu eixo horizontal. Aplicando-se a transformação de Schwarz-Christoffel (e.g. Eq. 4.2.5), sobre o semi-plano $\zeta$, tem-se:

$$
\text { Zeta }=\frac{\ln \left(\frac{1}{2}\left(\zeta_{2}-\zeta_{1}\right) Z+\frac{1}{2}\left(\zeta_{1}+\zeta_{2}\right)\right)-\frac{1}{2}\left(\ln \zeta_{1}+\ln \zeta_{2}\right)}{\frac{1}{2}\left(\ln \zeta_{2}-\ln \zeta_{1}\right)}
$$

Por sua vez a transformação inversa de Zeta para Z é dada pela Eq. 4.3.19.

$$
Z=\frac{\exp \left(\frac{1}{2}(1-\text { Zeta }) \ln \mathrm{z}_{1}\right) \exp \left(\frac{1}{2}(1+\text { Zeta }) \ln \mathrm{z}_{2}\right)-\frac{1}{2}\left(\mathrm{z}_{1}+\mathrm{Z}_{2}\right)}{\frac{1}{2}\left(\mathrm{z}_{2}-\mathrm{Z}_{1}\right)}
$$

Definidos os planos adimensionais que mapeiam o elemento sobre o eixo horizontal, a transformação de Schwarz-Christoffel (e.g. Eq. 4.2.5) pode ser aplicada a elementos estacionários de maneira bastante conveniente. Considere-se a definição de elementos lineares dada na Eq. 2.3.11, reescrita a seguir:

$$
\Omega=-\left(\frac{1}{i}\right) \frac{1}{2 \pi} \int_{-1}^{1} \frac{\mu(\Delta)}{Z-\Delta} d \Delta
$$

onde a presença do termo $\left(\frac{1}{i}\right)$ define um linedoublet e sua ausência, um linedipole. Duas condições devem ser observadas na construção de um elemento analítico unifilar: 1) a distribuição de esforços deve produzir valores reais e 2) a função obtida deve produzir uma descontinuidade no potencial $(\Omega)$ ao longo do elemento. Definindo-se a variável de integração $\Xi$ ao longo do eixo do plano Zeta, tem-se:

$$
\Omega=-\left(\frac{1}{i}\right) \frac{1}{2 \pi} \int_{-1}^{1} \frac{\mu(\Xi)}{Z-\Delta(\Xi)} \frac{d \Delta}{d \Xi} d \Xi
$$


Reescrevendo-se a integral acima conforme procedimento utilizado na seção 2.3.2 (Eq. 2.3.12), tem-se:

$$
\Omega=\left(\frac{1}{i}\right) \frac{\mu(\text { Zeta })}{2 \pi} \int_{-1}^{1} \frac{1}{Z-\Delta} d \Delta+\left(\frac{1}{i}\right) \frac{1}{2 \pi} \int_{-1}^{1} \frac{\mu(\text { Zeta })-\mu(\Xi)}{Z-\Delta(\Xi)} \frac{d \Delta}{d \Xi} d \Xi
$$

Novamente à semelhança do procedimento utilizado na seção 2.3 .2 a integral do primeiro termo da expressão é resolvida produzindo-se (ver Eq. 2.3.13):

$$
\Omega=\left(\frac{1}{i}\right) \frac{\mu(\text { Zeta })}{2 \pi} \ln \frac{Z-1}{Z+1}+\left(\frac{1}{i}\right) \frac{1}{2 \pi} \int_{-1}^{1} \frac{\mu(\text { Zeta })-\mu(\Xi)}{Z-\Delta(\Xi)}\left(\frac{d \Delta}{d \Xi}\right) d \Xi
$$

Diferentemente, entretanto, da Eq. 2.3.13, o núcleo da integral da Eq. 4.3.23, não produz polinômios, mas uma expressão transcendental de difícil manipulação. Alternativamente aos procedimentos de integração usuais, é recomendado o uso de fórmulas de quadraturas que fornecem valores aproximados para a integral (Wendland, 2005) ${ }^{14}$. Observe-se ainda que, embora o segundo termo da Eq. 4.3.23 não seja polinomial, o núcleo da integral utiliza o mesmo polinômio de esforços $\mu$. Assim, a o potencial fica expresso em termos de apenas um polinômio em vez de dois como em modelos de elementos retilíneos (ver Eq. 2.3.16).

A derivada $\frac{d \Delta}{d \Xi}$ (Eq. 4.3.23) é dada a partir do mapeamento dado na Eq. 4.3.19 pela seguinte expressão:

$$
\frac{d \Delta}{d \Xi}=\frac{\ln z_{2}-\ln z_{1}}{z_{2}-z_{1}} \exp \left(\frac{1}{2}(1-\Xi) \ln z_{1}+\frac{1}{2}(1+\Xi) \ln z_{2}\right)
$$

Definidos os termos que formam a integral da Eq. 4.3.23, a quadratura é aplicada na forma gaussiana:

\footnotetext{
${ }^{14}$ Informação fornecida por Edson Wendland durante discussões cotidianas
} 


$$
I_{n}=\int_{a}^{b} f(x) d x \approx \sum_{i=1}^{n} A_{i} f\left(x_{i}\right)
$$

onde $A_{i}$ são coeficientes de ponderação e $x_{i}$ são pontos onde a quadratura é calculada. Para polinômios de ordem menor ou igual a $2 n-1$, obtém-se os $n$ pontos e coeficientes para os quais a quadratura de maneira exata. Stroud e Secrest (1966, pág. 100) fornecem tabelas de pontos e coeficientes para a utilização da quadratura gaussiana. Assim, a Eq. 4.3.23 se torna expressa por:

$$
\Omega=\left(\frac{1}{i}\right) \frac{\mu(Z e t a)}{2 \pi} \ln \frac{Z-1}{Z+1}+I_{n}
$$

onde $I_{n}$ é a aproximação da quadratura gaussiana para a integral da Eq. 4.3.23.

Conforme já visto na equação Eq. 2.3.17, a expansão do termo logarítmico em parte real e imaginária produz uma descontinuidade do potencial sobre o eixo $X$ (parte real do plano $Z$ ). No caso de elementos retilíneos o elemento repousa sobre o eixo $X$ (Figura 4.3.3a) e a função $\mu(Z)$ é utilizada para ponderar a descontinuidade de acordo com o desejado. Elementos curvilíneos e sua distribuição dos esforços, por outro lado, não coincidem com o eixo $X$ (Figura 4.3.3b).

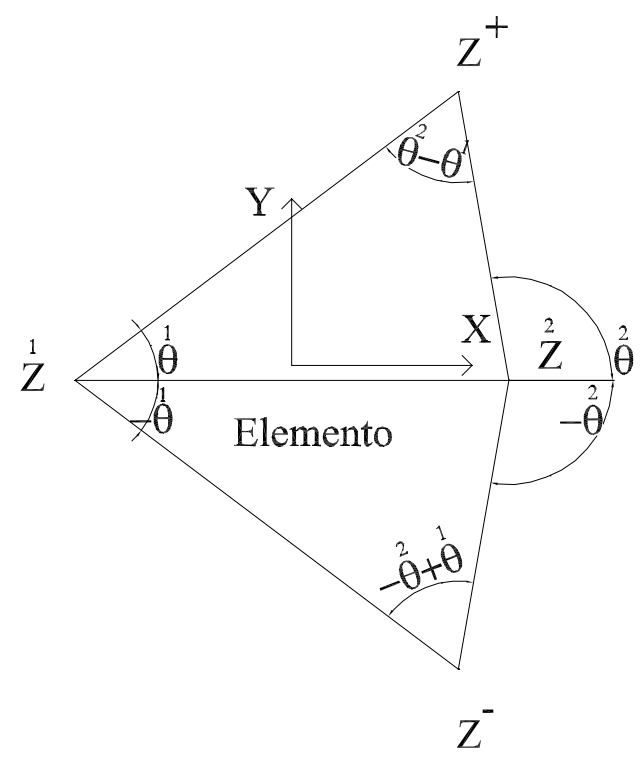

(a)

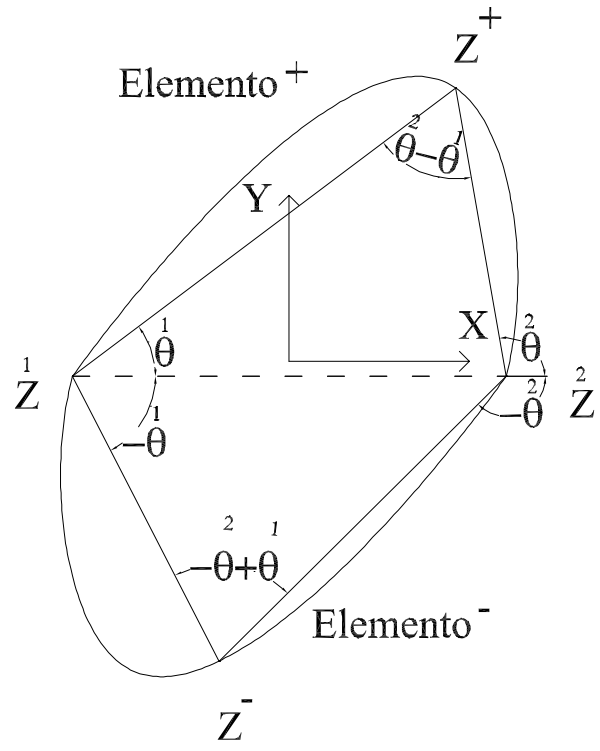

(b)

Figura 4.3.3 - Esquema de correção do ramo do elemento curvilíneo 
O ângulo $\theta$ é definido por $\theta=\arg \left(Z-{ }^{2}\right)-\arg (Z-\stackrel{1}{Z})$, assim, próximo ao eixo $X$ $\theta^{+}=\pi$ e $\theta^{-}=-\pi$. Dessa forma, o ângulo $\theta$ pode ser corrigido para que a descontinuidade ocorra entre os lados de cima e de baixo do elemento. Definindo-se $\mathfrak{I} Z<0 \cap \Im$ Zeta $>0$ a região abaixo do elemento e acima do eixo $X$ e $\mathfrak{I} Z>0 \cap \Im$ Zeta $<0$ a região abaixo do eixo $X$ e acima do elemento, tem-se:

$$
\begin{aligned}
& \mathfrak{I} Z<0 \cap \Im \text { Zeta }>0\left\{\begin{array}{l}
\Omega^{+}=-\left(\frac{1}{i}\right) \frac{\mu(X)}{2 \pi}\left[\ln \frac{X-1}{X+1}-i \theta+2 \pi i\right]+I_{n} \\
\Omega^{-}=-\left(\frac{1}{i}\right) \frac{\mu(X)}{2 \pi}\left[\ln \frac{X-1}{X+1}-i \theta\right]+I_{n}
\end{array}\right. \\
& \mathfrak{I} Z>0 \cap \mathfrak{I Z e t a}<0\left\{\begin{array}{l}
\Omega^{-}=-\left(\frac{1}{i}\right) \frac{\mu(X)}{2 \pi}\left[\ln \frac{X-1}{X+1}+i \theta-2 \pi i\right]+I_{n} \\
\Omega^{+}=-\left(\frac{1}{i}\right) \frac{\mu(X)}{2 \pi}\left[\ln \frac{X-1}{X+1}-i \theta\right]+I_{n}
\end{array}\right.
\end{aligned}
$$

Resultados obtidos da simulação de um único elemento linesink utilizando-se cinco pontos de quadratura tabelados em Stroud e Secrest (1966) são apresentados sobre o plano $\zeta$ na Figura 4.3.4.
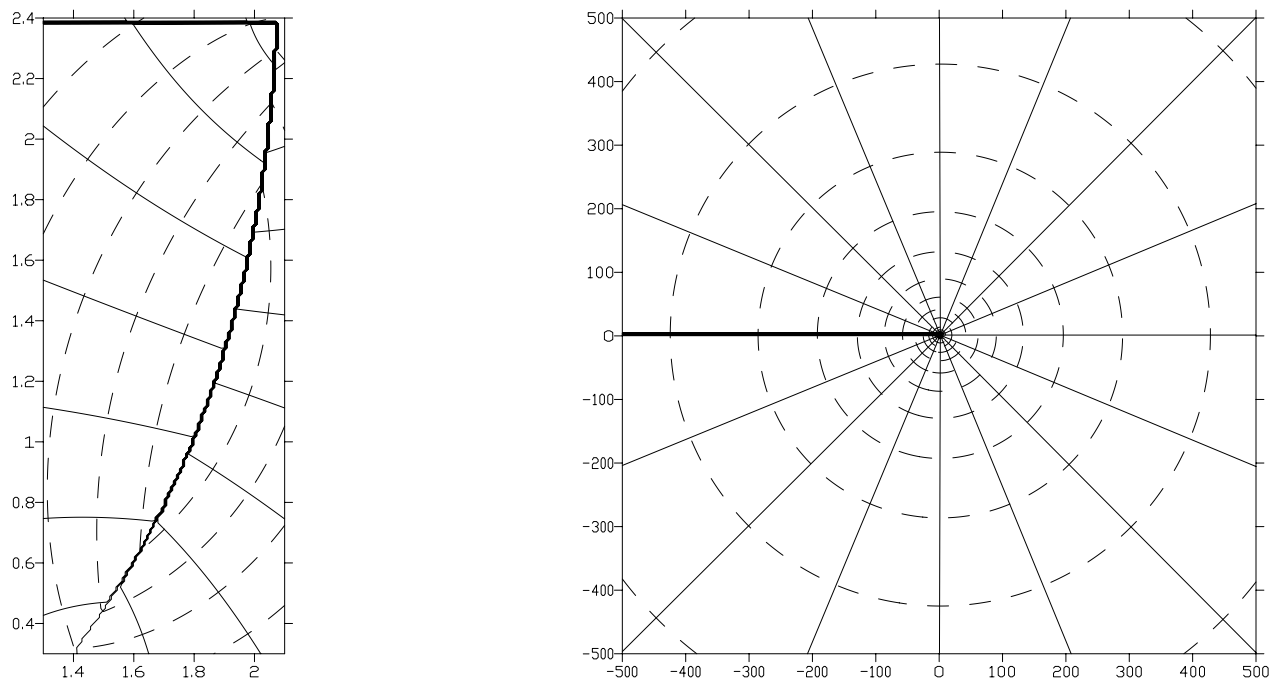

Figura 4.3.4 - Distribuição de potencial (linhas tracejadas) e de corrente (linhas contínuas) devido a um linesink. À esquerda, distribuição próxima ao elemento. À direita, distribuição distante do elemento. 


\subsection{Implementação Computacional}

Uma vez que diversas funções analíticas estão implementadas de forma a satisfazer condições de contorno em fronteiras internas de aqüíferos, são, por sua vez, implementados processos para a especificação de condições de contorno sobre suas fronteiras externas. São discutidas nesta seção as rotinas computacionais, escritas em Python, para a inclusão do efeito da recarga direta de Aqüíferos Longos, geração de Imagens e o mapeamento de SchwarzChristoffel. As rotinas do Método de Imagens e da transformação de Schwarz-Cristoffel (seção 4.2) foram desenvolvidas originalmente neste trabalho e encontram-se disponíveis no $\mathrm{CD}$ em anexo. A rotina de recarga de Aqüíferos Longos utiliza as fronteiras posicionadas no exterior de domínios como um argumento para a construção do objeto aqüífero. Se apenas uma fronteira é declarada, é utilizado apenas o Método de Imagens, se uma segunda fronteira é inclusa, os elementos são adicionados ao modelo mediante as transformações de SchwarzChristoffel.

\subsubsection{Classes CoastalAquifer}

A classe de aqüíferos CoastalAquifer é especificada a fim de gerenciar o modelo de acordo com as necessidades de ambos métodos (Método de Imagens e transformações de Schwarz-Christoffel). Seguindo a implementação de objetos analíticos descrita na seção 4.4, os objetos CoastalAquifer (ver Figura 4.4.1) podem ser estacionários ou transientes.

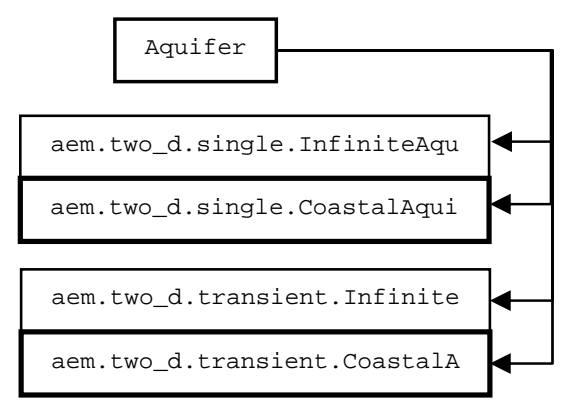

Figura 4.4.1 - Classes de aqüíferos adicionadas ao programa

A função de um objeto aqüífero, como já discutido, é introduzir parâmetros e efeitos regionais e gerenciar o modelo construído. Em aqüíferos cujas fronteiras estiverem dispostas paralelamente torna-se possível analisar o efeito da recarga direta sobre o modelo. Assim, além dos parâmetros especificados para os aqüíferos infinitos (InfiniteAquifer), é 
adicionado um quarto parâmetro para a especificação da recarga. A recarga especificada em um objeto aqüífero estacionário é, portanto, uniforme no espaço e ao longo do tempo. A recarga direta passada para o objeto aqǘfero transiente pode conter uma lista de pares de valores de instante e intensidade (ver seção 3.5.4).

A especificação de contornos externos (Método de Imagens) e os métodos de transformação de domínio (Schwarz-Christoffel) são inicializados no programa a partir do procedimento que preenche a lista de elementos do modelo (addElement). Dado o elemento que está sendo adicionado ao modelo e as fronteiras externas especificadas no objeto aqüífero, suas coordenadas podem ser transformadas, o que ocorre no caso de haver mais de um segmento de reta na fronteira. Em seguida, o elemento é submetido ao Método de Imagens considerando-se o semi-plano para o qual o elemento foi mapeado. Em casos com apenas uma fronteira, o elemento é submetido apenas ao Método de Imagens.

\subsubsection{Transformações de Domínio}

Conhecidas as expressões que relacionam um domínio qualquer a um semi-plano, sua implementação é especificada de modo a abranger cada caso. Assim, são definidas duas funções de transformação: map e mapback, responsáveis respectivamente pela transformação de um semi-plano no domínio aqüífero e a transformação inversa. São argumentos das funções as linhas do domínio considerado e os pontos a serem mapeados (Figura 4.4.2a). Em virtude de haver mais de uma possibilidade de semi-plano de mapeamento em função dos tipos de condições de contorno impostas, é necessário que a linha passada para a função de mapeamento seja um espelho. Assim, caso ambos "espelhos" sejam do mesmo tipo, o domínio é mapeado para o semi-plano horizontal. Caso contrário, o mapeamento é feito no semi-plano quadrante.

\subsubsection{Classes de Espelhos}

Para a implementação do Método de Imagens, é fundamental que: 1) cada ponto do setor originário encontre correspondência no setor imagem; 2) os elementos construídos como imagem expressem a relação dos esforços na imagem em função do tipo de "espelho". O processo é implementado por intermédio de classes para a construção de dois objetos: "espelhos" e imagens. 
A classe MirrorLine é especificada de forma bastante simples. A classe especifica, por meio de herança, as propriedades e procedimentos especificados na classe Line (ver Figura 4.4.2b).

\begin{tabular}{|c|c|c|}
\hline \multirow{3}{*}{ transform } & map & {$[$ geometry.two_d.Line],[geometry.two_d.Points] } \\
\cline { 2 - 3 } & & {$[$ geometry.two_d.Points] } \\
\cline { 2 - 3 } & mapback & {$[$ geometry.two_d.Line], [geometry.two_d.Points] } \\
\cline { 3 - 3 } & & {$[$ geometry.two_d.Points] } \\
\hline
\end{tabular}

a)

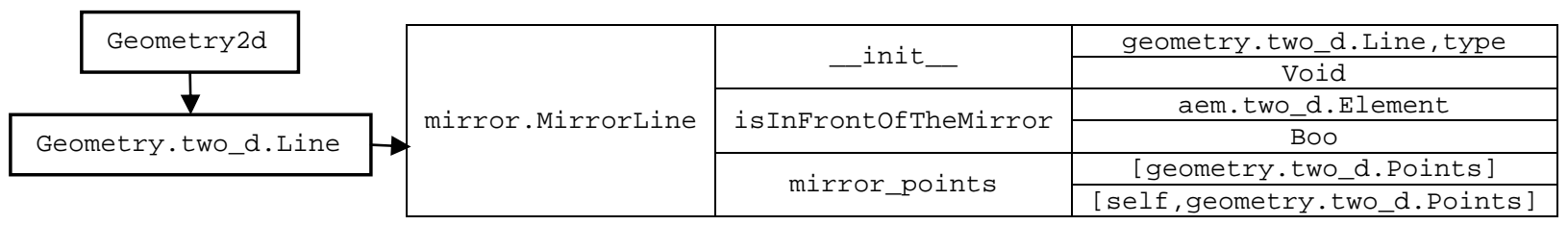

b)

\begin{tabular}{|c|c|c|}
\hline \multirow{4}{*}{ image } & \multirow{2}{*}{ image_method } & {$[$ aem.two_dElement], [mirror.MirrorLine] } \\
\cline { 2 - 3 } & \multirow{2}{*}{ image_model } & {$[$ aem.two_d.Elements],mirror.MirrorLine } \\
\cline { 2 - 3 } & & [image.Image] \\
\cline { 2 - 3 } & image_element & Element,MirrorLine \\
\cline { 3 - 3 } & & Image \\
\hline
\end{tabular}

c)

Figura 4.4.2 - Rotinas implementadas para as transformações de Schwarz-Christoffel e o método de imagens.

O objeto "espelho" é especificado, ainda, de modo a possuir uma propriedade designada para a sua identificação. Desta forma, as imagens podem ser geradas de acordo com a propriedade que caracteriza o tipo de fronteira requisitada no domínio do modelo. Dois procedimentos são especificados na classe MirrorLine: isInFrontofTheMirror e mirror_points. O primeiro destina-se a responder a seguinte pergunta: o ponto está em frente ao espelho? Retornando um valor booleano (TRUE ou FALSE). O segundo submete uma lista de pontos requisitados ao primeiro procedimento e em caso de obter o valor TRUE para todos os pontos, são obtidos os pontos localizados simetricamente do lado oposto ao espelho. O processo de construção de imagens é ilustrado no diagrama de blocos da Figura 4.4.3. As especificações para as classes de imagens são discutidas na seção a seguir. 


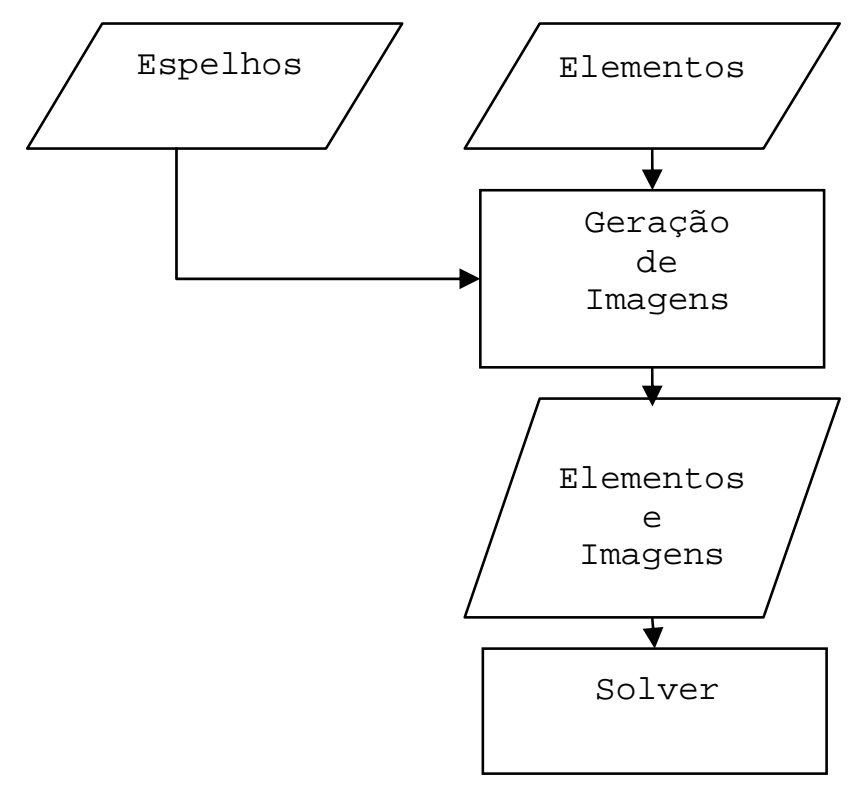

Figura 4.4.3 - Fluxograma de modelagem a partir de imagens

\subsubsection{Classes de Imagens}

Um objeto imagem especifica, por intermédio de herança, todas as propriedades e procedimentos do objeto analítico originário, tanto estacionário como transiente. Não obstante a herança obtida do objeto originário, um objeto imagem possui propriedades e procedimentos peculiares. Entre imagens e elementos, alguns procedimentos idênticos são os procedimentos de influência e os procedimentos de contribuição. A construção de uma imagem e os procedimentos de solução, no entanto, ocorrem de maneira diferenciada. Por exemplo, os objetos imagens não participam da lista de elementos do aqǘfero e sim do elemento posicionado à frente do "espelho".

Dadas as diferenças entre objetos imagens e objetos analíticos, é especificada uma classe Image fundamental, contendo os procedimentos e parâmetros necessários à construção de um objeto do gênero. Assim, conforme discutido na seção 4.3, objetos imagens são construídos a partir de um objeto analítico originário e dos objetos "espelhos" utilizados no modelo (Figura 4.4.3).

A expressão Eq. 4.3.5 define a obtenção de um elemento imagem em função do seu elemento de origem e de um espelho. Em função do tipo de "espelho" é requisitada a construção de objetos ImageDirect ou ImageInverse para cada elemento refletido. Uma vez que não é possível determinar a priori a classe de elemento a ser recebida pela imagem como herança, a especificação da classe é feita por intermédio de uma função denominada 
image_element no módulo image (ver Figura 4.4.2c). O módulo contém três funções destinadas a desempenhar tarefas preliminares à construção do objeto imagem: a) image_method,b) image_model e c) image_element.

a) Função image_method

A função image_method implementa a estrutura do método. Essa função é responsável pela verificação da simetria das fronteiras do domínio do modelo e pela utilização da função image_model para cada espelho, podendo repetir o processo de acordo com a necessidade de recursão e o número de imagens necessário.

b) Função image_model

A função image_model, por sua vez, expõe os elementos ao "espelho" solicitado e retorna as imagens produzidas por intermédio da função image_element. Submetido o elemento ao "espelho", o procedimento mirror_points invoca o procedimento isInFront0fTheMirror para a verificação da posição dos elementos em relação ao espelho. Caso os elementos estejam posicionados na frente do "espelho", ou seja, se as ordenadas em relação ao eixo definido pelo "espelho" forem positivas, são localizados os pontos eqüidistantes aos do elemento real na direção normal ao espelho.

c) Função image_element

Para construir um objeto imagem de maneira fiel ao objeto originário é necessário identificar a classe pela qual o objeto foi construído, de modo a obter desta classe sua herança (objetos não produzem herança). A classe do objeto originário é identificada pesquisando-se na lista de propriedades dir ( ) dos pacotes aem. single e aem.transient a classe de quem o objeto analítico é instância. As classes ImageDirect e ImageInverse encontram-se dentro do escopo da própria função image_element que instancia as imagens. Assim, a função image_element tem o objetivo de retornar objetos imagens para um dado objeto de origem e um "espelho" (objeto da classe MirrorLine). 


\subsubsection{Procedimento de Construção de Objetos Imagens}

Uma imagem pode ser implementada como todas as propriedades e métodos de um objeto analítico (ver seção 3.5.2). O procedimento de construção, compartilha (herda) todos os processos especificados para um objeto analítico, no entanto, adicionalmente especifica uma propriedade extra destinada para a identificação imediata de qual objeto "espelho" a originou. Imagens originadas de objetos analíticos de parâmetros conhecidos têm seus parâmetros pré-determinados pela relação $\underset{e l}{\stackrel{e}{\Theta}}$ (ver Eq. 4.3.6 a Eq. 4.3.15) durante o procedimento de construção.

A determinação de parâmetros desconhecidos, por sua vez, é também obtida por intermédio das relações $\underset{e l}{\stackrel{e}{\Theta}}$. No entanto neste caso, a relação não é empregada no procedimento de construção, mas nos procedimentos de solução.

\subsubsection{Procedimentos de Solução nos Objetos Imagens}

Os procedimentos de solução nas classes de imagem são herdados da classe de elementos. Assim eles são denominados da mesma forma que na seção 3.5.3e. No entanto, apenas os procedimentos de obtenção de termos da matriz de coeficientes (getMatrixCoefficients) e o procedimento de atribuição dos resultados obtidos do sistema para as devidas imagens (getParameters) são especificados como é descrito a seguir.

Nota-se que, por se tratar de relações lineares, a relação entre ambos $(\underset{e l}{\stackrel{e}{\Theta}})$ pode ser expressar apenas os termos de esforços (ver Eq. 3.2.4) de todas imagens produzidas em conseqüência de um elemento qualquer, exceto as imagens geradas para condições de contorno do terceiro tipo (ver Apêndice C). De acordo com a notação já utilizada nas Eq. 3.2.4 e Eq. 4.3.16 escreve-se o potencial devido a um elemento $i$ e suas $N$ imagens da seguinte forma:

$$
\Phi_{i}=\lambda_{i} \boldsymbol{\Lambda}_{i}+\sum_{k=1}^{n}\left(\Theta \lambda_{i}\right) \Lambda_{k}
$$


O termo entre parênteses representa o conjunto de parâmetros da imagem $k$ obtido da relação $\Theta$ e do conjunto de parâmetros de seu originário $(\lambda)$. Isso permite que os objetos imagens tenham seus procedimentos de influência especificados apenas por intermédio de herança a partir dos objetos originários e que seus parâmetros de esforços sejam determinados em função apenas dos parâmetros do objeto originário. Por outro lado, cria-se a necessidade de se especificar procedimentos de solução que expressem (implementem) a relação desejada entre os parâmetros da imagem e do elemento originário (Eq. 4.3.6 a Eq. 4.3.15).

A expressão Eq. 4.3.16 descreve um termo da matriz de coeficientes de maneira que o esforço sobre o elemento aparecem sempre em evidência. $O$ posicionamento de cada termo da expressão, por sua vez é realizado pelo procedimento getMatrixCoefficients especificado nos objetos analíticos (objetos originários das imagens) utilizando a lista de imagens geradas a partir dele próprio. Assim, cada função de influência passa a ser multiplicada pela relação $\Theta$ correspondente e somada constituindo-se no valor do coeficiente que multiplica um único termo desconhecido. Durante o processo de construção da matriz, o procedimento getMatrixCoefficients de cada objeto analítico passa a ser responsável pela aquisição e soma dos termos obtidos para cada imagem do objeto (contidos na sua lista de elementos). Portanto o procedimento getMatrixCoefficients de um objeto imagem retorna o valor obtido de seu procedimento de influência (seção 3.5.3b) multiplicado pela relação $\Theta$ (Eq. 4.3.16) que guarda com seu objeto originário.

Durante a simulação de escoamentos transientes, o procedimento getMatrixCoefficients dos objetos analíticos é especificado de forma semelhante aos objetos estacionários. Uma vez que cada objeto transiente possui objetos do tipo Unitstep, a lista de elementos elementList é percorrida duas vezes. Na primeira, como discutido na seção 4.5.4, é retornado o termo referente ao último elemento Unitstep da lista e incluído no somatório. No segundo laço, o procedimento reconhece somente os objetos imagens e invoca deles os procedimentos getMatrixCoefficients incluindo-os no somatório do coeficiente desejado.

A determinação dos parâmetros de esforços de uma imagem, por sua vez, é, da mesma forma que os objetos analíticos, baseada nas diferenças obtidas para o parâmetro (ver seção 3.5.3e). O procedimento de transmissão dos resultados aos objetos imagem, à semelhança do que ocorre nos objetos analíticos, é especificado de forma a realizar o processo inverso de getMatrixCoefficients. Ou seja, o elemento imagem recebe a solução do objeto parent e 
multiplica pela relação $\Theta$ obtendo a sua própria solução. Em seguida, a solução obtida é passada para as eventuais imagens deste elemento.

\subsection{Exemplo de Aplicação}

Batista et al. (2004) apresentam modelos obtidos mediante o método de imagens em domínios alongados segundo as diretrizes de Keller (1953). O método é aplicado em problemas hipotéticos bastante simples, produzindo resultados confiáveis. No entanto, a metodologia utilizada no trabalho oferece limitações computacionais para problemas realistas (Batista et al., 2004). Na metodologia utilizada por Batista et al. (2004), a representação dos contornos regionais do aqüífero é feita utilizando-se apenas o Método de Imagens, sendo necessária uma série infinita de imagens. No entanto, Batista et al. (2004) utilizam uma quantidade de quarenta reflexões recursivas, truncando a série (o artigo encontra-se no CD anexo). Segundo Bischoff (1981), em casos onde são observadas duas fronteiras paralelas do mesmo tipo, a série de imagens converge lentamente. Em problemas onde se apresentam diferentes tipos de contornos, a convergência ocorre é mais acelerada. Batista et al. (2004) utilizam para estes modelos apenas três imagens. Esta representação não satisfaz de maneira exata a condição de contorno. $\mathrm{O}$ erro, assim admitido, cresce à medida que a posição da fronteira se distancia dos elementos utilizados no modelo.

Transformações de Schwarz-Christoffel, por sua vez, exigem apenas uma imagem em casos de fronteiras paralelas de mesmo tipo (ver Figura 4.2.1b). Em casos onde são apresentados diferentes tipos de fronteiras são necessárias três imagens para uma representação exata do semi-plano (ver Figura 4.2.1c). A seguir é discutido um modelo hipotético de aqüífero constituído por fronteiras de tipos diferentes. O problema é simulado com o programa $\operatorname{Tim}^{\mathrm{SL}}$ e as rotinas da seção anterior. O arquivo de entrada utilizado para esta simulação encontra-se disponível no CD em anexo.

Considere-se a presença de um único lago retangular na escala local que possui interação direta com o aqüífero (Figura 4.5.1, à esquerda), extraindo deste uma vazão conhecida e constante. Considere-se, ainda, a presença de uma região onde o aqüífero apresenta uma condutividade hidráulica reduzida delimitada por uma região poligonal. $\mathrm{Na}$ Figura 4.5.1 (à esquerda) são ilustradas a localização e configuração do lago e as fronteiras do aqüífero onde ele está inserido. As fronteiras do aqüífero são caracterizadas por uma linha onde o potencial é conhecido, onde é imposta a condição do primeiro tipo (dreno) e outra linha onde não existe descarga transversal, onde é imposta a condição do segundo tipo 
(parede). Na Figura 4.5.1 (à direita) apresenta-se a disposição obtida para o lago e suas imagens no semi-plano de Schwarz-Christoffel.
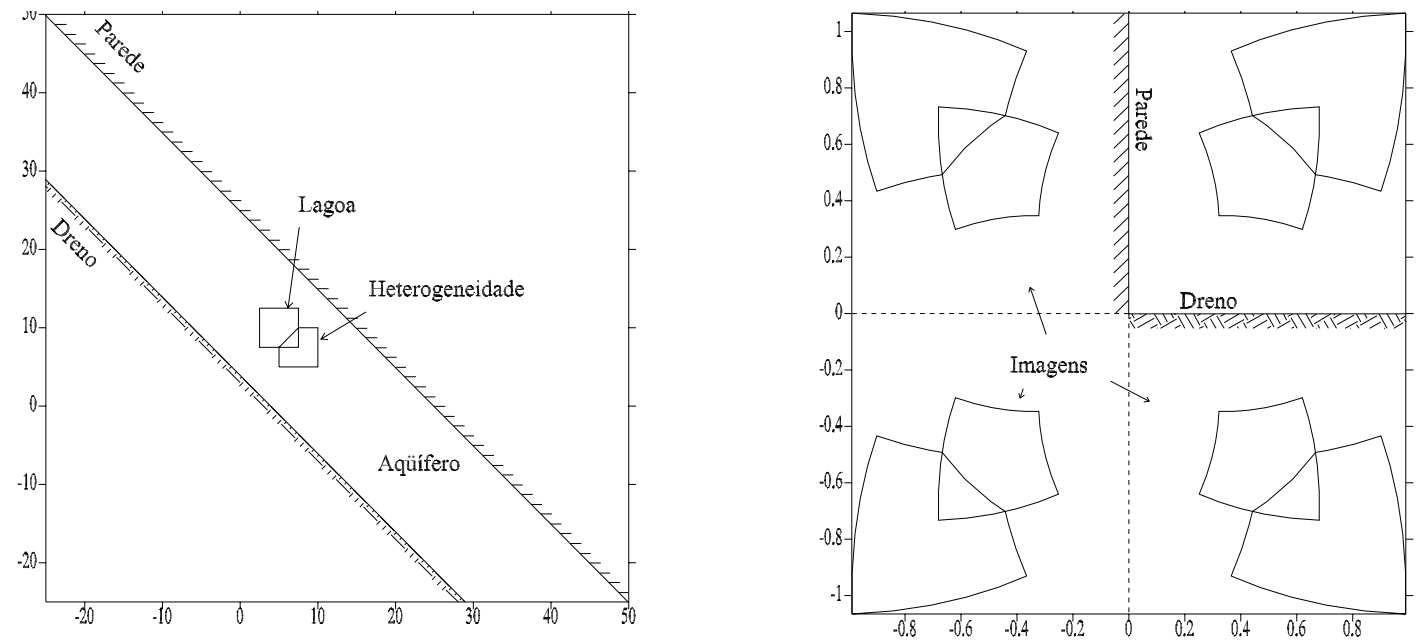

Figura 4.5.1 - Transformação de domínio para um Aqüífero Longo.

Considerando-se unidades arbitrárias de dimensão [L] e tempo [T], os dados do aqüífero são idealizados da seguinte forma: condutividade hidráulica observada na maior parte do aqüífero unitária $(1 \mathrm{~L} / \mathrm{T})$; recarga devido à precipitação no valor de $10^{-4} \mathrm{~L} / \mathrm{T}$; cota topográfica da base do aqǘfero no valor de $-1 \mathrm{~L}$ e a cota piezométrica observada ao longo do exutório do aqüífero igual a zero, tornando o potencial de descarga $(\Phi)$ também nulo (zero $\mathrm{L}^{3} / \mathrm{T}$ ). A condutividade da heterogeneidade encontrada no aqüífero é a centésima parte daquela observada na maior parte $(0,01 \mathrm{~L} / \mathrm{T})$.

Dois cenários são apresentados. No primeiro, o balanço da lagoa observado no aqüífero é nulo, onde a recarga é igual à descarga em direção à fronteira drenante, e da mesma forma a lagoa, onde a lâmina precipitada é igual à evaporada no espelho de água. No segundo, a chegada da estação seca é concebida de tal forma que a recarga direta do aqüífero é anulada e as perdas atmosféricas da lagoa superam as precipitações em $0,01 \mathrm{~L}^{3} / \mathrm{T}$, equivalente a uma lâmina de $4 \cdot 10^{-4} \mathrm{~L} / \mathrm{T}$.

A representação do escoamento esperado no aqüífero para essas condições é obtida utilizando-se a sobreposição dos elementos analíticos adequados para os contornos apresentados no interior do aqüífero (lagoa e heterogeneidade) e a representação de fronteiras externas é feita mediante as técnicas de transformação de domínio e de geração de imagens. A fim de se evitar a sobreposição de pontos de controle, a poligonal que define a heterogeneidade atravessa o interior da poligonal da lagoa. 
Os elementos são utilizados com o comprimento unitário $(L=1 \mathrm{~L})$ nas faces de cada polígono, totalizando-se trinta e nove elementos retilíneos mais a recarga regional (40 elementos). O matriz de solução do modelo possui uma quantidade de linhas igual à de colunas (sem sobrespecificação) atingindo-se ordem 119. Considerando-se o computador utilizado para a simulação (IBM PC, processador Celeron 1GHz, RAM 256MB, S.O. Microsoft Windows), embora uma matriz pequena, o tempo consumido pelo o interpretador Python 2.1 na montagem da matriz é de 34,802s e na solução do sistema linear é de $0,05386 \mathrm{~s}$. O tempo consumido para a tarefa não é pequeno e não representa uma vantagem em relação a implementações de outros métodos de simulação de escoamento subterrâneo. Entretanto, é possível acreditar em reduções $90 \%$ do tempo computacional utilizando-se linguagens de alto desempenho.

A distribuição de cargas piezométricas obtida para o primeiro cenário é ilustrada na Figura 4.5.2 em uma ampla faixa do domínio e detalhada próximo aos elementos na Figura 4.5.3a. Observe-se que as condições de escoamento impostas sobre ambas fronteiras do aqüífero são satisfeitas. 


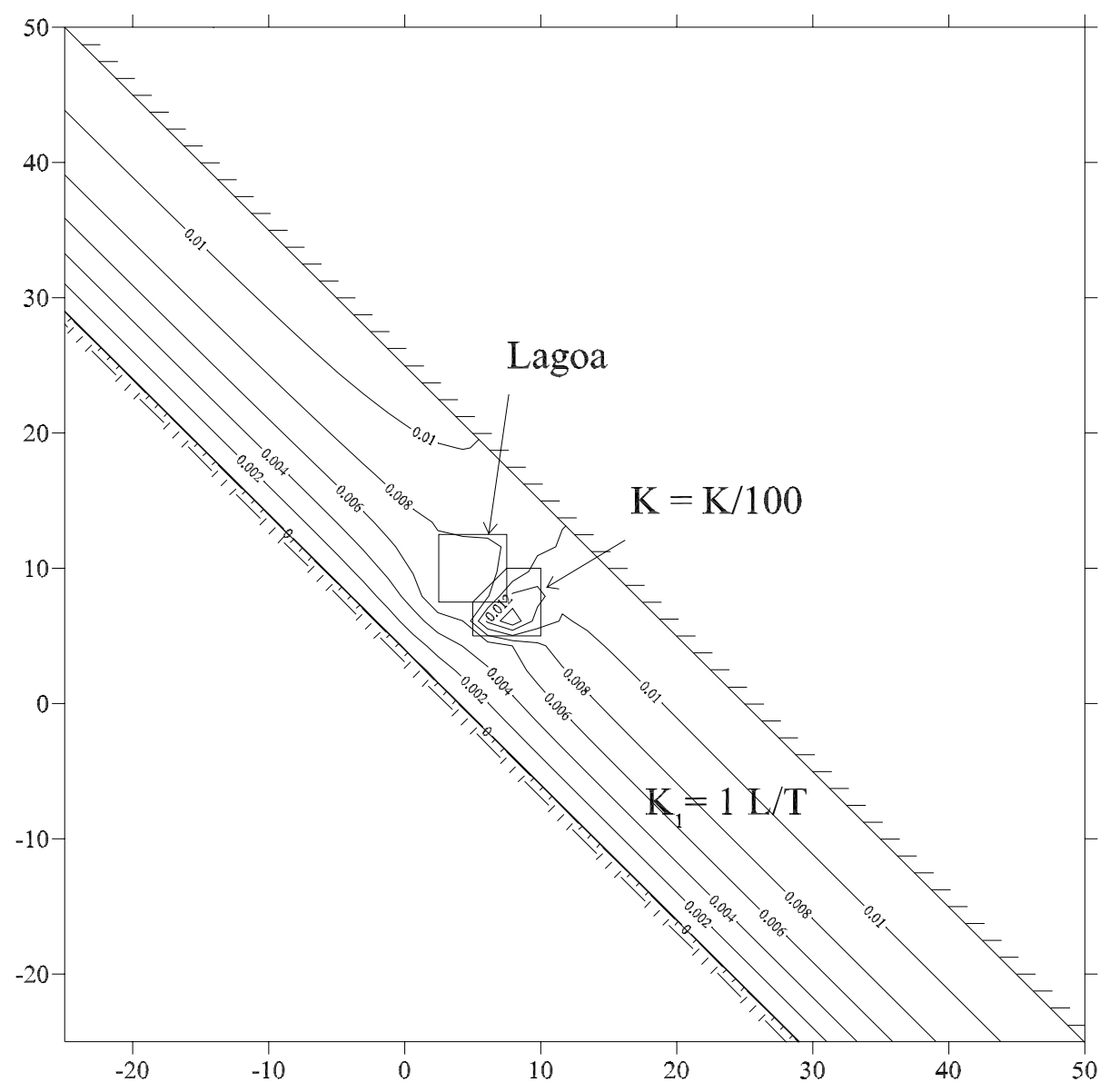

Figura 4.5.2 - Distribuição de equipotenciais para o aqüífero

O segundo cenário é modelado de forma semelhante. No entanto, os elementos transientes são empregados mediante apenas o Método de Imagens. O intervalo de tempo com o qual é feito o cálculo dos esforços é definido utilizando-se $\Delta \mathrm{t}=\frac{\mathrm{L}_{\min }^{2}}{\alpha}$ (devido a Zaadnoordijk e Strack, 1993), onde $L_{\min }$ é o comprimento mínimo dos elementos utilizados no modelo $(L=1 \mathrm{~L})$ e $\alpha$, a difusividade hidráulica do aqüífero. O tempo computacional é a soma do consumido na solução do modelo estacionário mais o tempo necessário para a sobreposição dos efeitos ao longo de 50 passos de $\Delta t$, totalizando-se $18.360 \mathrm{~s}$.

A evolução das cargas piezométricas é, então, obtida conforme ilustrado nas Figura 4.5.3. Observa-se que as condições de contorno são mantidas ao longo de ambas fronteiras do aqüífero. No entanto, ocorre o desvio dos potenciais em relação ao especificado à medida que se afasta da região dos elementos, devido ao truncamento da série de imagens dos elementos transientes. 

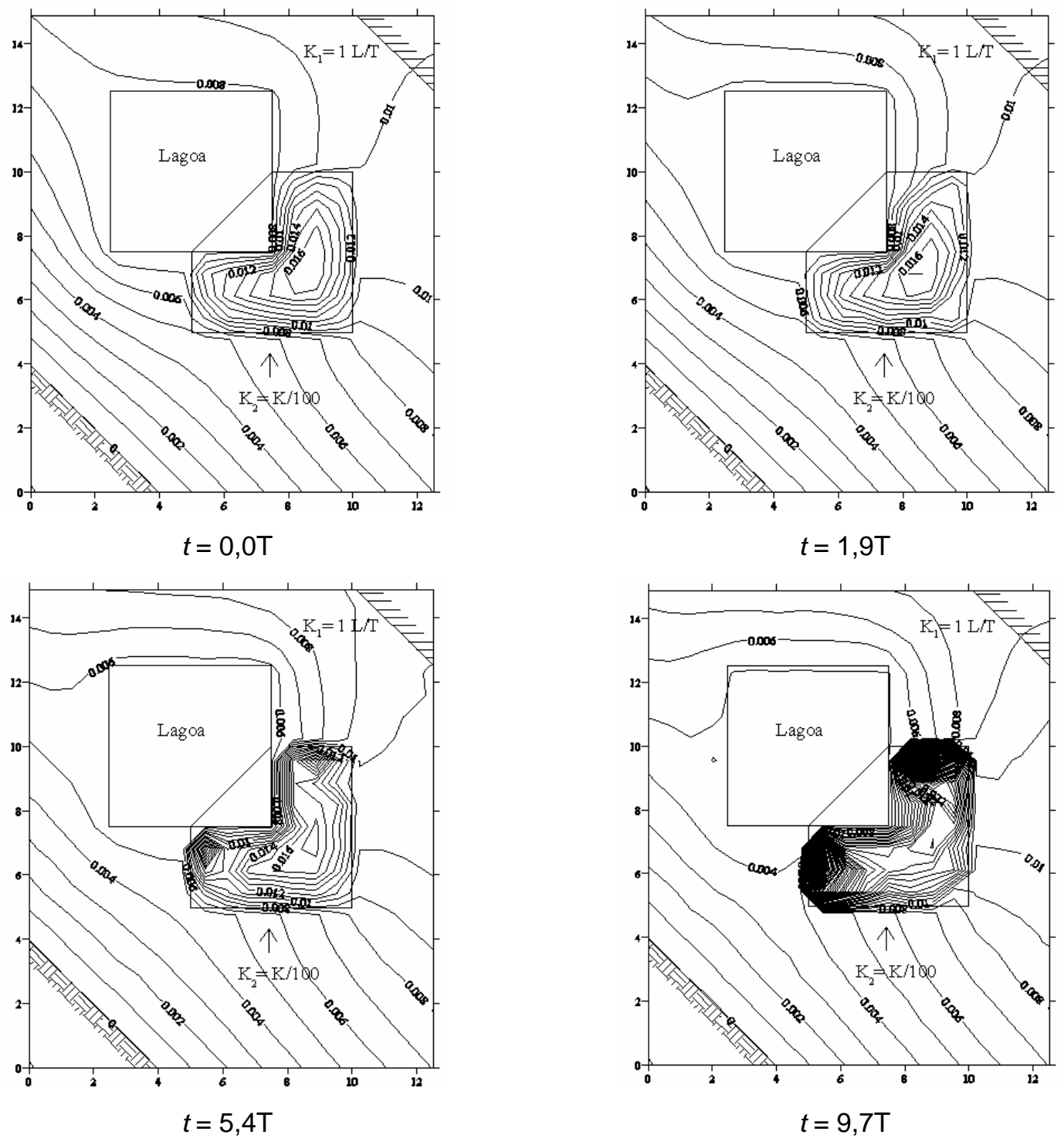

Figura 4.5.3 - Detalhe da distribuição de equipotenciais em torno do lago. Em cada instante de tempo $(t)$ as cargas piezométricas tornam-se menores.

A solução obtida nos elementos satisfaz as condições especificadas para seus respectivos contornos. Observe-se que a carga piezométrica é mantida constante ao longo da borda do lago. Ao longo das bordas da heterogeneidade são observadas, igualmente, a continuidade e a suavidade das cargas piezométricas. 


\section{Capítulo 5}

\section{IMPACTO DA CAPTAÇÃO DA ADUTORA AGRESTE/TRAIRI/POTENGI SOBRE A LAGOA DO BONFIM}

Neste capítulo, os elementos analíticos são aplicados ao estudo do escoamento subterrâneo em uma região utilizada como fonte de captação de água do Sistema Adutor Agreste/Trairi/Potengi, no Rio Grande do Norte. A captação do sistema é feita de forma mista sobre o Aqüífero Barreiras e sobre a Lagoa do Bonfim. O Aqüífero Barreiras é caracterizado como Aqüíferos Longos e seu escoamento, natural e induzido pela captação, são modelados tanto em regime permanente como transiente. Embora o impacto do sistema sobre a Lagoa tenha motivado diversos estudos, o presente estudo contribui para a questão fornecendo uma calibração dos parâmetros hidráulicos e simulando cenários de seca na região.

\section{1 Área de Estudo}

A área de estudo encontra-se sobre uma pequena faixa do Aqüífero Barreiras (parte superior da Figura 5.1.1), localizada na costa leste do Estado do Rio Grande do Norte. A Lagoa do Bonfim possui, aproximadamente, um volume igual a $83 \mathrm{~km}^{3}$ e uma área de espelho de água igual a $8 \mathrm{~km}^{2}$ e é a maior do Sistema Lacustre Bonfim (parte inferior da Figura 5.1.1), formado por seis lagoas localizadas na $24^{\mathrm{a}}$ zona UTM entre as coordenadas $252 \mathrm{kmE}$, $9325 \mathrm{kmN}$ e $263 \mathrm{kmE}, 9335 \mathrm{kmN}$.

O Aqüífero Barreiras constitui-se no principal manancial subterrâneo do Estado, fornecendo atualmente cerca de 12.803,60 $\mathrm{m}^{3} / \mathrm{h}$ para abastecimento humano. Até o ano de 2020, é estimado ainda um acréscimo de $4.550,54 \mathrm{~m}^{3} / \mathrm{h}$, ou seja de $35 \%$, sobre a atual demanda do aqǘffero (Hidroservice, 1998). O Sistema Adutor Agreste/Trairi/Potengi, por sua vez, foi projetado em 1996 para atender uma demanda de $1.628,35 \mathrm{~m}^{3} / \mathrm{h}$, até 2016 , captandose água, na região da Lagoa do Bonfim, por meio de poços tubulares e bombeamento direto da Lagoa.

No entanto, a operação do sistema tem ocasionado redução do volume de água nas lagoas da região (Pereira et al., 2002). Estudos atuais têm procurado avaliar qual é a capacidade de produção do sistema adutor, dado o nível mínimo do espelho de água da Lagoa 
do Bonfim, protegido por decisão judicial. Batista (2001) avalia que a capacidade do sistema é aproximadamente $50 \%$ abaixo do esperado para o horizonte de projeto. Com o iminente aumento de demanda previsto para a segunda fase do projeto, novos estudos têm sido feitos em busca de alternativas de locais para a instalação de novas baterias de poços (e.g. Manuel Filho e Castro, 2002).

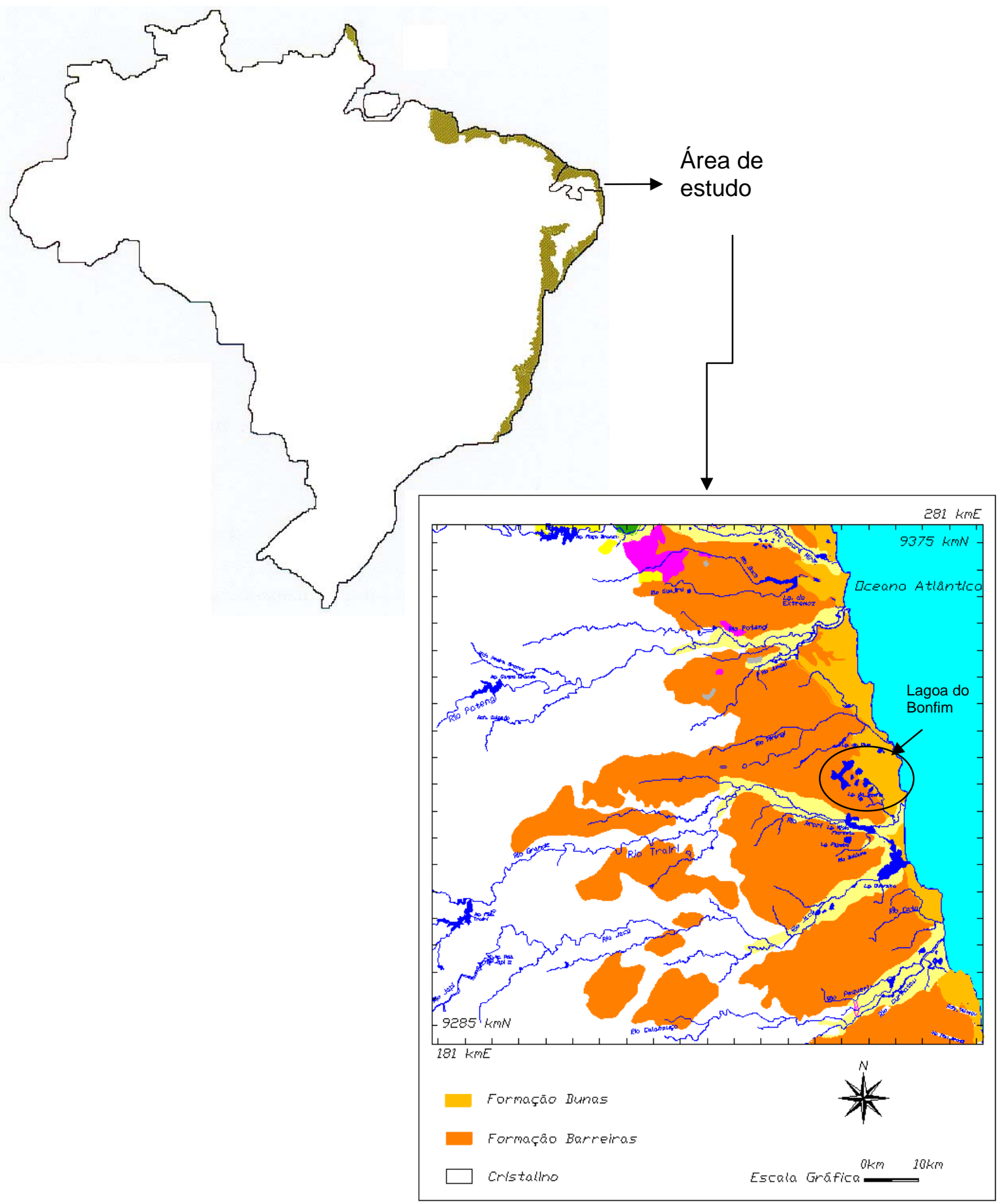

Figura 5.1.1 - Localização do Aqüífero Barreiras (acima) e da Área de Estudo (abaixo). (Modificado de FGV, 1998 e Hidroservice, 1998) 


\subsubsection{Clima e Pluviometria}

Predominam no Estado os climas muito quente e semi-árido (BSw'h', na classificação de Köppen), com estação chuvosa no final do verão e tropical chuvoso (As', na classificação de Köppen) com estação seca durante o verão e chuvosa durante o outono e inverno. A pluviometria anual em todo litoral leste tem médias entre 1200 a $1500 \mathrm{~mm}$ (decrescente na direção do interior do Estado) e a evapotranspiração potencial é estimada em 1600mm/ano.

\subsubsection{Recursos Hídricos}

O litoral do Rio Grande do Norte é marcado pela presença dos exutórios de bacias hidrográficas que nascem no sertão e agreste do Estado e diversas pequenas bacias de escoamento difuso que ocorrem nas proximidades de exutórios das bacias maiores (ver Figura 5.1.2). No litoral leste do Estado, oito sub-bacias de escoamento difuso formam a Bacia 16, com uma área total de $649,4 \mathrm{~km}^{2}$. O Sistema Adutor Agreste/Trairi/Potengi encontra-se na Sub-bacia 16-6, que possui uma área de apenas $187,7 \mathrm{~km}^{2}$. A Sub-bacia está localizada entre as bacias dos rios Pirangi (Bacia 9) e Trairi (Bacia 10), com áreas de 458,9 e 2.867,4 km², respectivamente (Hidroservice, 1998).

\subsubsection{Geologia}

A maior parte do litoral nordestino, portanto também a área de estudo, tem o seu embasamento rochoso formado por uma seqüência arqueozóica (cristalino), sendo constituída basicamente de gnaisses, granitos gnáissicos e calcários cristalinos. Sobre esse embasamento, toda a faixa central do Rio Grande do Norte apresenta o afloramento de uma seqüência geológica do período cretáceo composta de arenitos na parte inferior e calcário no topo, pertencentes ao Grupo Apodi (também conhecido como Jandaíra). No litoral, no entanto, o Grupo Apodi encontra-se encoberto por sedimentos recentes.

As formações sedimentares que ocorrem ao longo de todo litoral do Rio Grande do Norte pertencem ao Grupo Barreiras, formado por sedimentos terciários e quaternários (era cenozóica). Os sedimentos marinhos terciários recebem o nome de Formação Barreiras. Essa Formação recebe ainda uma cobertura de depósitos eólicos recentes (quaternário), chamados Dunas. 


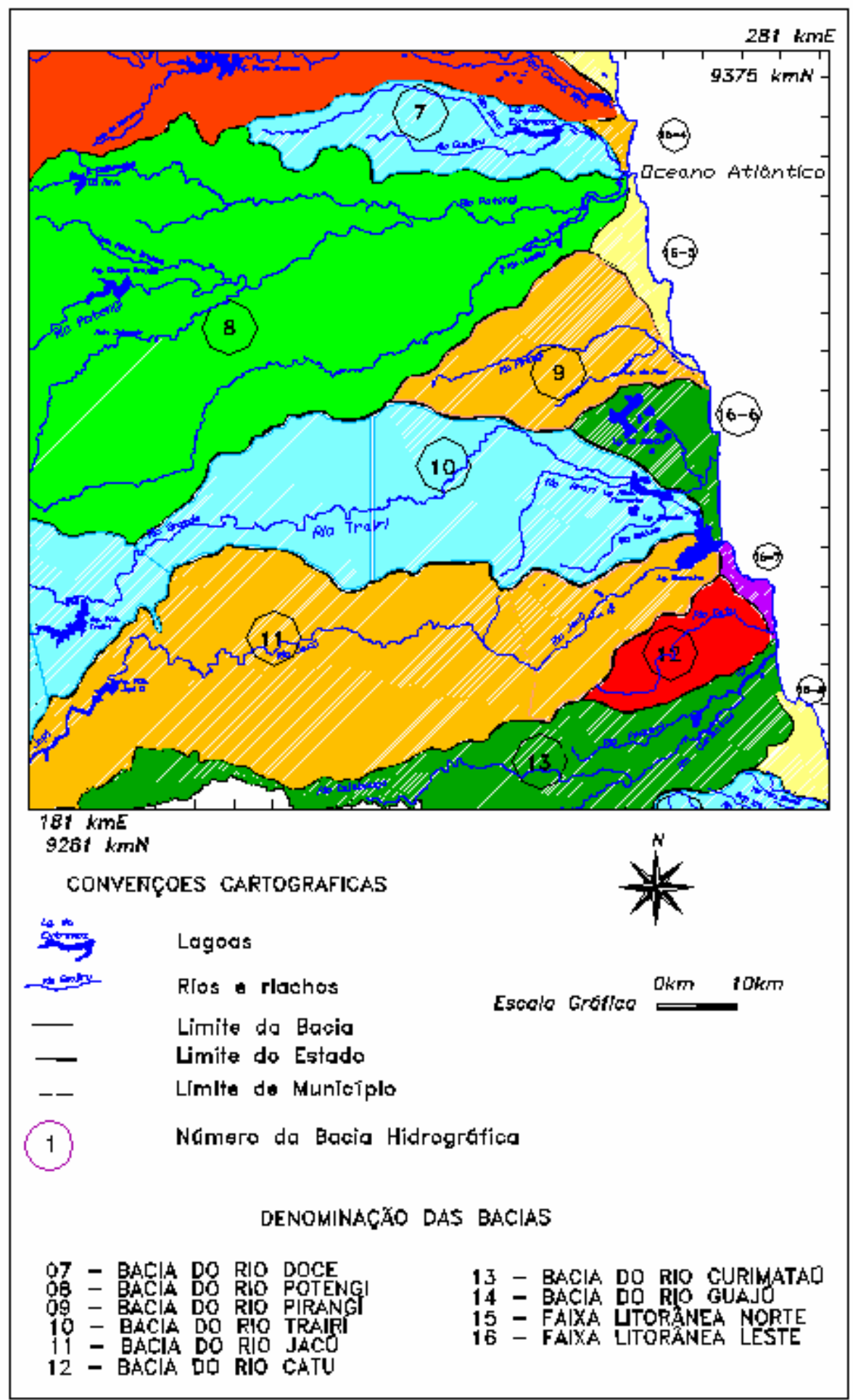

Figura 5.1.2 - Localização da Bacia Hidrográfica do Sistema Lacustre Bonfim (Bacia 16-6) e bacias vizinhas. (Modificado de Hidroservice, 1998). 
A presença das coberturas dunares da região é caracterizada, segundo Pereira (2001), por três camadas. A primeira, as neodunas, que se encontram próximas à linha de costa, constituídas de areias eólicas quartizosas brancas. A segunda é formada pelas paleodunas, dunas antigas compostas de areias eólicas bem selecionadas em cores avermelhadas e amareladas. Por fim, os vales dos rios, ocupados por aluviões de cascalho, limos, siltes e argilas. No domínio das pequenas lagoas do sistema Bonfim, porém, caracteriza-se uma interface arenosa entre os sedimentos eólicos e os não-eólicos, denominada lençóis eólicos, de cor branco-acinzentada com granulometria fina. Essas camadas ocorrem em depressões sobre as quais se instalaram algumas lagoas, entre elas o Sistema Lacustre Bonfim conforme apresentado na Figura 5.1.3 (Pereira et al., 1996).

O afloramento da Formação Barreiras aparece superficialmente à oeste da Formação Dunas e estende-se até sua fronteira com o afloramento da bacia sedimentar inferior, a cerca de $11 \mathrm{~km}$ da Lagoa do Bonfim conforme a legenda apresentada na Figura 5.1.3 (Lacerda, 1999 apud Pereira, 2001) ${ }^{15}$. A Formação Barreiras, nesta região, ocorre com sedimentos heterogêneos pouco consolidados, fracamente selecionados, caracterizando-a pela presença de arenitos conglomeráticos, a partir da base, seguidos de arenitos argilosos relativamente homogêneos com significativas e freqüentes intercalações de lentes argilosas (IPT, 1982 apud Pereira et al., 1996) ${ }^{16}$. Alguns estudos caracterizam o aqüífero como semi-confinado, uma vez que, em seu topo, ocorrem lentes de argila (e.g. IPT, 1982 e Melo, 1995 apud Pereira, $2001)^{17}$. No entanto, a ocorrência dessas lentes é esparsa e não configura a presença de uma camada contínua, configurando-se um sistema aqǘfero único denominado Dunas/Barreiras (Pereira, 2001).

${ }^{15}$ Lacerda, A.MA. (1999). Caracterização Geológica e Hidrogeológica da Região de Monte Alegre - RN. Monografia de Graduação. 83p. Universidade Federal do Rio Grande do Norte. Natal.

${ }^{16}$ Instituto Paulista de Tecnologia (1992). Estudo Hidrogeológico Regional Detalhado do Estado do Rio Grande do Norte. Secretaria da Indústria e Comércio do Rio Grande do Norte. Vol. 1.

${ }^{17}$ Melo, J.G. (1995). Impactos do Desenvolvimento Urbano nas Águas Subterrâneas de Natal - RN. Tese de Doutorado. 196p. Universidade de São Paulo. São Paulo. 


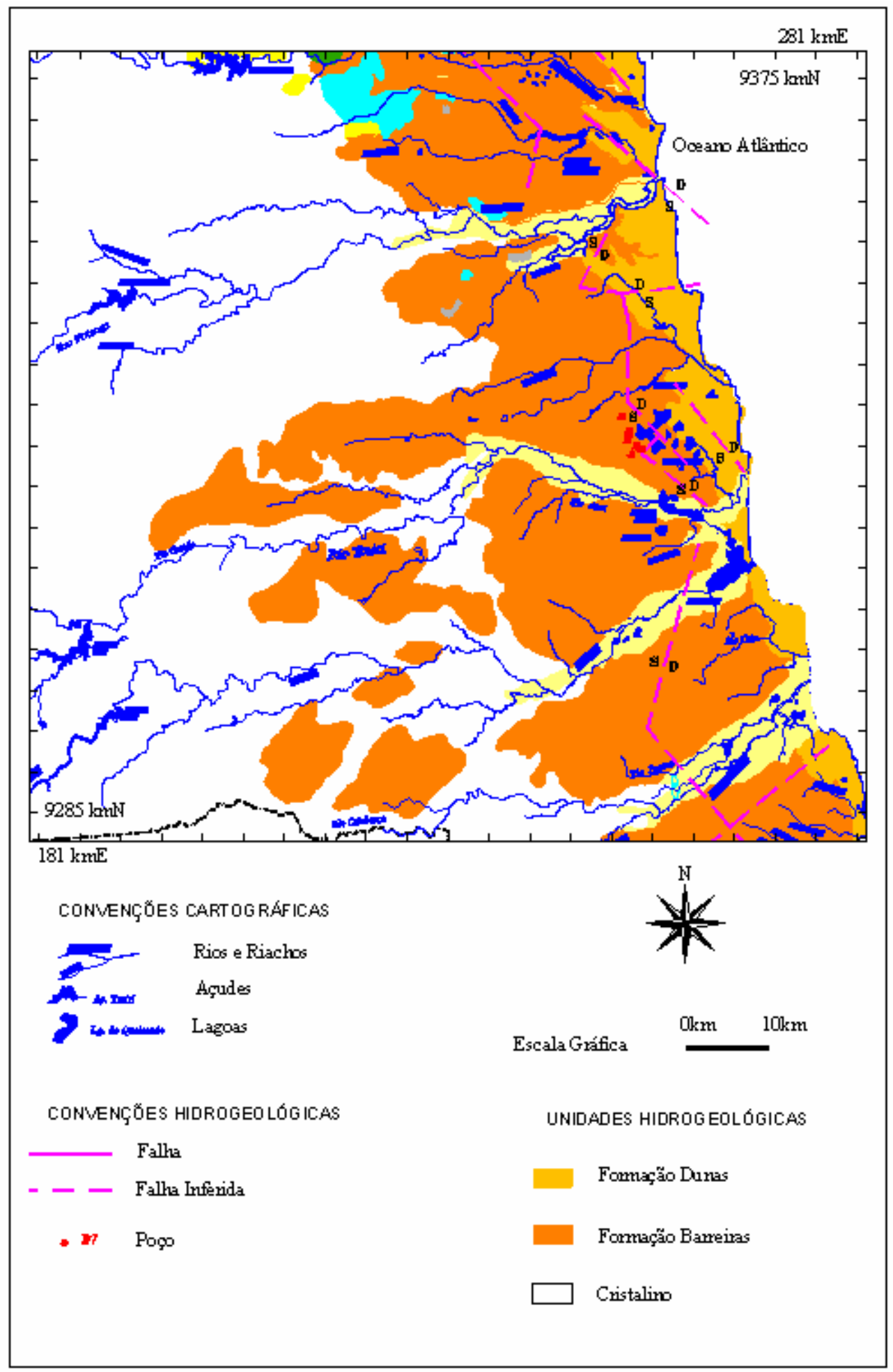

Figura 5.1.3 - Unidades Hidrogeológicas da Área de Estudo (Modificado de Hidroservice, 1998). 


\subsubsection{Uso e Ocupação do Solo}

A região do Sistema Lacustre Bonfim possui claramente uma distinção na sua cobertura de solos. Ocorrem dois tipos básicos de solo na região cuja distribuição, ou fronteiras seguem a orientação da linha costeira. Os solos que constituem a cobertura da parte leste da região são latossolos vermelho amarelo, originados do afloramento dos sedimentos areno-argilosos do Grupo Barreiras e por isso são solos profundos. Na parte leste da região a cobertura é dada pelas areias quartizosas também considerados profundos originados dos depósitos dunares (SUDENE, 1971, apud Pereira, 2001) ${ }^{18}$.

A ocupação vegetal ocorre basicamente sob a forma de Florestas Ombrófilas (Matas de Dunas e Mata Ciliar - rasas e densas) e Floresta Semicaducifólia (florestas de tabuleiros arbóreas). Essa denominação é referente às condições climáticas reinantes. A região do Sistema Lacustre Bonfim apresenta vegetação ombrófilas concentradas à leste, sobre as coberturas dunares e floresta de tabuleiros sobre os latossolos. Essa ocupação vegetal é caracterizada, portanto, pela presença de estratos arbóreos (como cajueiros e mangueiras) e herbáceos (arbustos). Na Figura 5.1.4 é ilustrada a ocupação encontrada na área de estudo.

Segundo o RIMA (Relatório de Impactos ao Meio Ambiente) do projeto do Sistema Adutor Agreste/Trairi/Potengi, a área de estudo compreende áreas rurais, com ocupações distribuídas pontualmente nas áreas cultivadas e duas sedes municipais: São José de Mipibu, atualmente com 20377 hab rurais e 14912mil hab urbanos e Nísia Floresta, com 8823 hab rurais e 8590 hab urbanos. A área cultivada na região abrange cerca de 1500 ha próximos à Lagoa Ferreira Grande e 600 ha nas proximidades da Lagoa do Bonfim dos quais cerca de 400 ha são irrigados contra menos de 1\% daqueles próximos à Lagoa Ferreira Grande. No entanto, as lagoas da região não têm um uso consuntivo local significativo. A população fixa nas áreas do entorno da Lagoa do Bonfim é de apenas 600 moradores que e próximo à Lagoa Boa Água um lugarejo com cerca de 150 moradores. A população flutuante é constituída pelos proprietários de chácaras e dos usuários de infraestruturas de lazer, como clubes e parques, instalados principalmente nas lagoas Carcará com 500 habitantes, Boa Água com 300 habitantes e a Lagoas do Bonfim com cerca de 340 habitantes. Todo abastecimento particular de água e feito a partir de poços e captação direta das lagoas.

18 Superintendência de Desenvolvimento do Nordeste (1971). Levantamento Exploratório: Reconhecimento de Solos do Estado do Rio Grande do Norte. Bol. Téc. no 21, 532p. Ministério da Agricultura. Recife. 


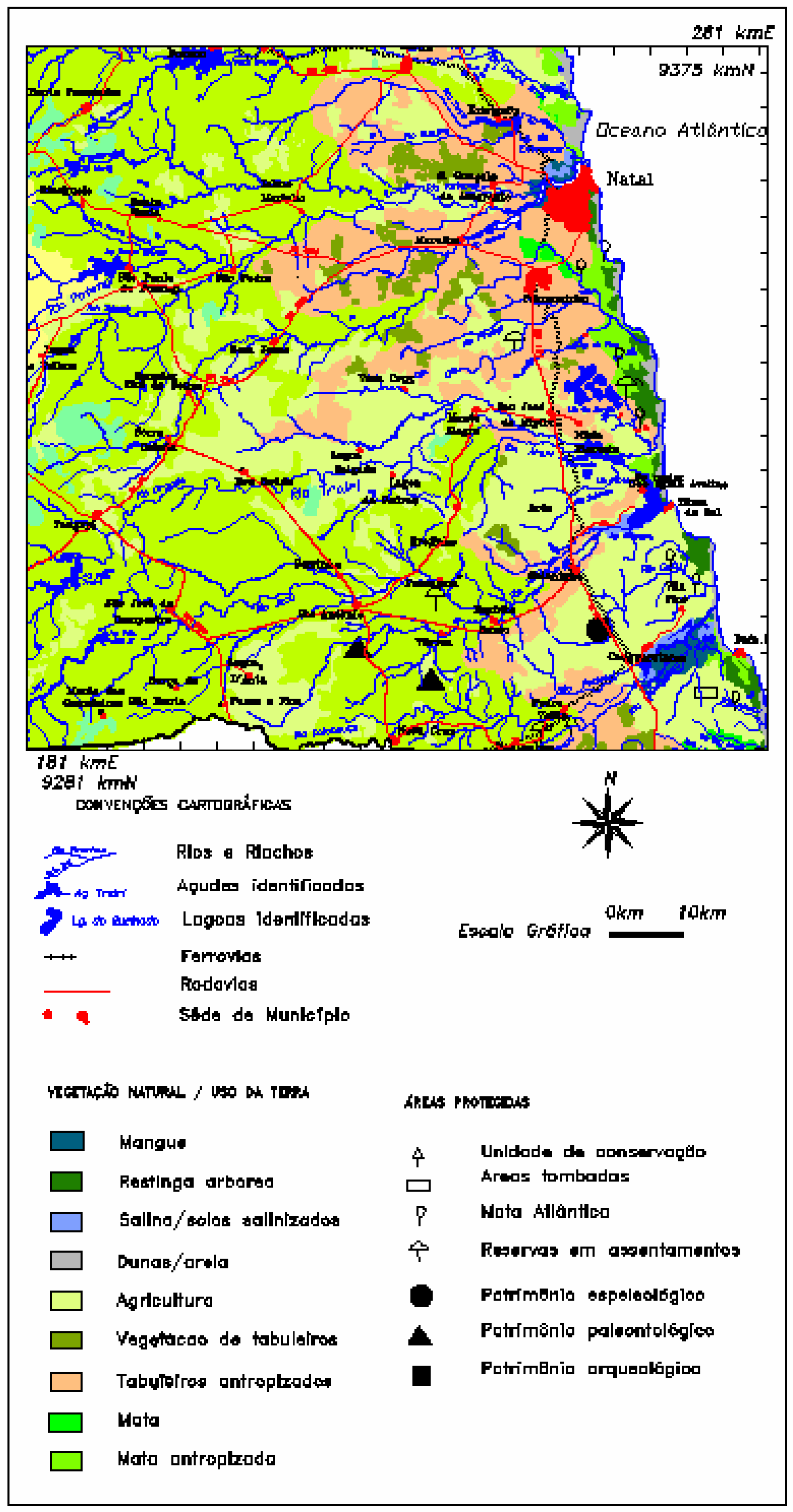

Figura 5.1.4 - Uso e Ocupação dos Solos da Área de Estudo (Modificado de Hidroservice, 1998) 


\subsubsection{Estudos Anteriores de Potenciometria na Área de Estudo}

Com o objetivo de caracterizar o comportamento hidráulico e hidrológico do Sistema Lacustre Bonfim, Pereira (2001) realizou uma intensiva campanha de monitoramento. A campanha foi realizada entre outubro de 1998 e dezembro de 2000 sob condições pluviométricas abaixo da média (ano de 1999, com $850 \mathrm{~mm}$ ) e acima da média (ano de 2000, com $2080 \mathrm{~mm})$.

A partir dos dados obtidos no final da estação chuvosa de 1999, Pereira (2001) confeccionou o seguinte mapa ilustrado na Figura 5.1.5. As linhas isopotenciométricas apresentadas no mapa foram obtidas manualmente pelo autor (Pereira, 2001). O trabalho identifica direções preferenciais do escoamento subterrâneo e divisores hidrogeológicos conforme representado na legenda. Foram identificadas as seguintes direções de escoamento: a entrada de água à oeste da Lagoa do Bonfim, saídas de água da Lagoa nos seus extremos norte e sul e, à leste da Lagoa, foram identificadas características sazonais do escoamento, em direção à Lagoa, na estação chuvosa, e contrária à Lagoa, durante a estiagem.

O estudo identifica, ainda, os seguintes divisores hidrogeológicos. No lado oeste da Lagoa, o escoamento dirige-se para três drenos: o Riacho Pium, o Rio Trairi e a Lagoa do Bonfim. No lado leste, o escoamento é direcionado pelas lagoas do sistema Bonfim, ainda pelos rios Pium e Trairi e principalmente pelos riachos Timbó e Boacica e pela costa. Os divisores e as principais direções de escoamento mencionadas podem ser encontrados na Figura 5.1.5. 


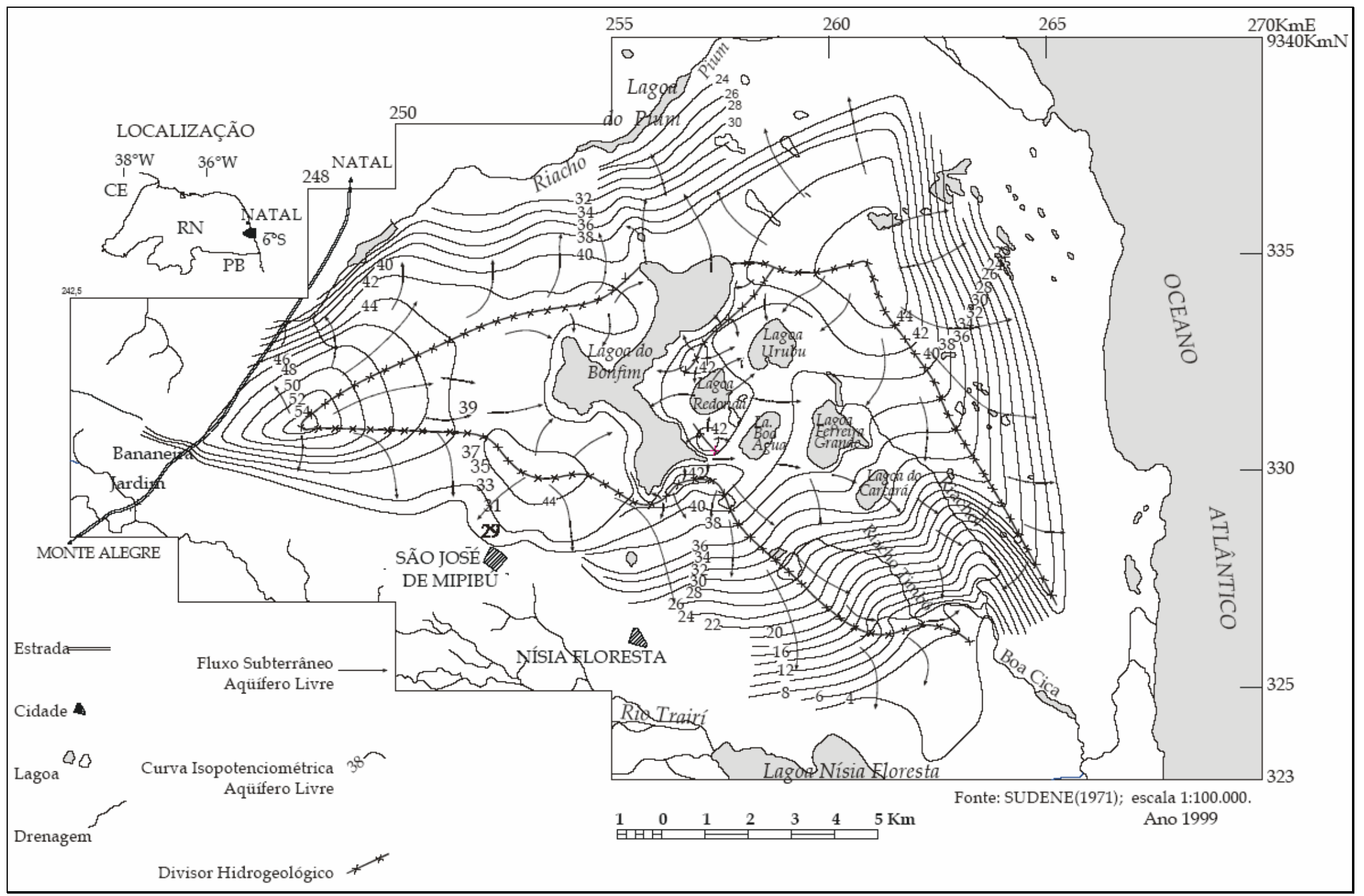

Figura 5.1.5 - Mapa potenciométrico do aqüífero livre da região do Bonfim - RN (Retirado de Pereira et al., 2003) 
Manuel Filho e Castro (2002) realizaram uma avaliação numérica das condições de exploração do aqüífero na área de estudo. A área simulada foi compreendida pela área entre o afloramento do cristalino (Sinal de Macaíba) à oeste, o Oceano Atlântico à leste, o Riacho Pium ao norte e o Rio Trairi ao sul. O modelo numérico de diferenças finitas é construindo considerando o aqüífero homogêneo e a interação do escoamento subterrâneo com os corpos hídricos da região. A abrangência do modelo é ilustrada na Figura 5.1.6.

As células utilizadas ao longo da linha de costa foram designadas a impor a condição do primeiro tipo (cota piezométrica igual a zero). A linha de afloramento do cristalino foi representada por condições do segundo tipo (fluxo através igual a zero). Riachos e lagoas foram modelados como contornos onde são impostas condições do terceiro tipo (cargas piezométricas e resistências transversais ao escoamento conhecidas). Por fim, os sete poços do sistema Agreste/Trairi/Potengi foram representados por células onde eram esgotadas vazões conhecidas.

Diversos autores avaliam condutividades diferentes na Formação Barreiras e na Formação Dunas (e.g. Melo e Feitosa, 1998; Pereira, 2001). No entanto, Manuel Filho e Castro (2002) homogeneízam a condutividade hidráulica em toda a área igual a $10,0 \mathrm{~m} / \mathrm{d}$, considerando o domínio homogêneo e isotrópico em todo o domínio da simulação. De modo semelhante, a cota do topo da formação cristalina ocorre, em média, com valores de $-30 \mathrm{~m}$ e $10 \mathrm{~m}$, respectivamente, sob as Dunas e sob as Barreiras. Porém, a variação deixa de ser significativa em relação às dimensões da área de estudo, sendo utilizado o valor de $-20 \mathrm{~m}$ em toda a área. A recarga direta do aqüífero devido às chuvas foi utilizada segundo estima Melo e Feitosa (1998) igual a $6,6.10^{-4} \mathrm{~m} /$ dia.

O sistema foi simulado considerando-se uma retirada de 130l/s dos poços mais 100l/s da Lagoa do Bonfim. Na Figura 5.1.6 é apresentado o resultado da simulação. Observa-se que a distribuição de cargas hidráulicas caracteriza a Lagoa do Bonfim influente para o aqüífero. $\mathrm{O}$ resultado diverge, ainda, do mapa potenciométrico obtido por Pereira (2001), não se identificando as características levantadas no trabalho anterior. 


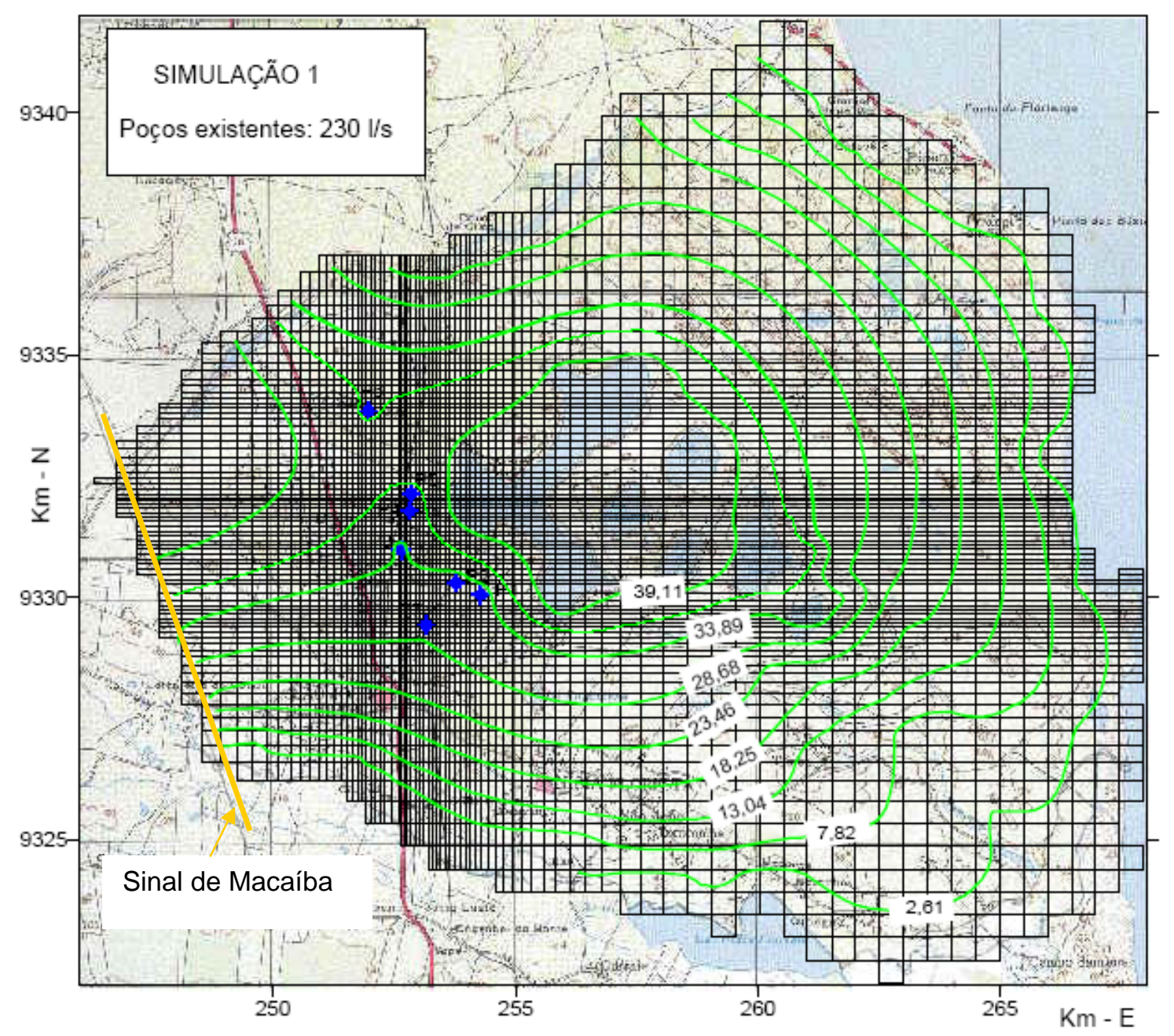

Figura 5.1.6 - Simulação da piezometria da região da Lagoa do Bonfim (Fonte: Manuel Filho e Castro, 2002)

\subsubsection{Estudos Anteriores de Impactos sobre a Lagoa do Bonfim}

A captação da adutora Agreste/Trairi/Potengi foi projetada inicialmente para retirar água apenas da Lagoa do Bonfim. Após a ocorrência do rebaixamento contínuo de 1,17m do nível de água em 9 meses (Nov/98 a Ago/99) de bombeamento da Lagoa (Pereira et al., 2002), foram investigadas alternativas para a mitigação dos impactos ambientais devidos à redução do volume de água na Lagoa, porém, ainda sem estudos que avaliem qual é sua eficácia. Estudos hidrogeológicos recentes concluíram pela perfuração de uma bateria de poços suplementares à oeste da Lagoa, entretanto, não foram encontrados estudos de avaliação da eventual mitigação dos impactos sobre a Lagoa do Bonfim. Atualmente, embora uma nova bateria de poços tenha sido instalada em uma região à leste do Sistema Lacustre 
Bonfim, estudos de avaliação de impactos se tornam necessários à medida que fornecem suporte ao processo de gestão dos recursos hídricos.

Pereira et al. (2002) desenvolveram um modelo de balanço hídrico de reservatórios com o objetivo de estudarem o impacto da captação da adutora sobre a Lagoa do Bonfim. Foram simulados dois cenários correspondentes às duas etapas de implantação do sistema. No primeiro cenário, o balanço da Lagoa é simulado sem a operação do sistema. No segundo, é simulada a operação prevista para o horizonte de projeto. Os autores demonstram que, no mesmo período observado (Nov/98 a Ago/99) o rebaixamento sem o bombeamento teria sido $48 \%$ menor e avaliam o projeto como "muito impactante". Por outro lado, o rebaixamento da Lagoa do Bonfim devido ao bombeamento da vazão final de projeto no mesmo período teria sofrido impacto ainda maior, com $71,3 \%$ a mais que o natural.

Embora a avaliação forneça o grau de participação do bombeamento no comportamento do nível de água da Lagoa, a metodologia utilizada no estudo permite apenas a avaliação de eventos passados. Batista (2001) estendeu a metodologia para ser utilizada em séries de dados sintéticos permitindo extrapolar a avaliação para cenários futuros. No entanto, a metodologia utiliza relações empíricas elaboradas para obter as variáveis envolvidas no balanço hídrico da Lagoa em função de variáveis hidrometeorológicas, não permitindo, por exemplo, a inclusão ou retirada de poços da região.

\subsection{Modelagem do Escoamento Subterrâneo e Calibração}

Modelos de escoamento permitem facilmente a inclusão ou retirada de poços, permitindo, portanto, a reconfiguração do sistema. Embora trabalhos anteriores tenham construído modelos de escoamento sobre a área de estudo (Manuel Filho e Castro, 2002), nesta seção é construído um novo modelo considerando-se diferenças de condutividade hidráulica no domínio. A interação do escoamento subterrâneo com águas superficiais, por sua vez, é representada por meio do balanço hídrico fornecendo uma avaliação da cota do espelho em função de diversos cenários. A modelagem a seguir utiliza, ainda, a metodologia de cálculo apresentada nos capítulos 3 e 4, contribuindo, assim, com mais um aspecto inédito no estudo dos impactos físicos do projeto.

\subsubsection{Modelo Conceitual}


O Aqüífero Barreiras estende-se por uma longa faixa que extrapola a área de estudo com largura aproximadamente constante e igual a $25 \mathrm{~km}$. As fronteiras panorâmicas do aqüífero, em toda sua extensão, são dados pela linha de costa e pelo afloramento do cristalino. O aqüífero é composto por duas formações (Barreiras e Dunas) depositadas homogeneamente sobre uma formação impermeável localizada aproximadamente à cota de $-20 \mathrm{~m}$ em relação ao nível do mar. O topo do aqüífero coincide com a topografia do terreno e a superfície potenciométrica aflora sobre o terreno através dos corpos de água.

Embora os diversos estudos de monitoramento apresentem variação espacial em suas estimativas de condutividades e espessuras no contexto da Formação Barreiras (e.g. Pereira, 2001), grande parte dos autores conclui pela homogeneidade hidráulica do meio. Por outro lado, diversos autores igualmente concluem pela homogeneidade do conjunto Dunas/Barreiras. Entretanto, dado que as variações espaciais citadas ocorrem em torno de valore diferentes nas Barreiras e nas Dunas, o modelo conceitual deste trabalho define, hidraulicamente, ambas formações de maneira distinta.

A entrada de água no sistema aqüífero, por sua vez, ocorre, predominantemente, por meio da infiltração direta de chuvas. A variabilidade da taxa de recarga ocorre em função dos diversos usos e ocupações praticados sobre o solo. No entanto, conforme características levantadas sobre a área, não são observadas presenças significativas de regiões impermeabilizadas, ou ainda de regiões irrigadas. Por outro lado, a presença de vegetação rasa e arbórea em áreas distintas, embora significativa, não possui estudos de evapotranspiração real. Assim, a recarga do sistema é definida uniformemente distribuída e será calibrada no modelo em função da potenciometria obtida em Pereira (2001).

\subsubsection{Base de Dados}

Estimativas de parâmetros hidráulicos do escoamento, das condições do escoamento na fronteira, etc. demandam atividades de monitoramento do aqüífero e seus contornos, bem como da hidrometeorologia e do comportamento de seus usuários (uso/ocupação dos solos). Os dados apresentados nesta seção constituem-se na base de dados utilizada na alimentação do modelo computacional propriamente dito.

No Rio Grande do Norte, diversos órgãos têm cumprido as tarefas de monitoramento dos seus sistemas hídricos. Por intermédio desses órgãos tem-se levantado a base de dados necessária para a construção do modelo computacional (Tabela 5.2.1). A base cartográfica deste trabalho foi obtida a partir do Plano Estadual de Recursos Hídricos (Hidroservice, 1998) 
e da Superintendência de Desenvolvimento do Nordeste (SUDENE, 1983a,b). O histórico dos volumes de água produzidos no sistema de captação foi obtido na Companhia de Águas e Esgotos do RN (CAERN, 2002) ${ }^{19}$, enquanto os dados de monitoramento foram obtidos na SERHID (2002) ${ }^{19}$. Os dados hidrometeorológico, por sua vez, foram coletados na Empresa de Pesquisa Agropecuária do Estado (EMPARN, 2002) ${ }^{19}$, na Universidade Federal do RN (Estação Climatológica da UFRN, 2002) ${ }^{19}$ e no Comando Aéreo de Treinamento de Parnamirim (CATRE, 2002) ${ }^{19}$.

Tabela 5.2.1 - Bases de dados utilizadas para o trabalho

\begin{tabular}{|c|c|c|c|c|}
\hline Tema & Base & Denominação & Autor & $\begin{array}{c}\text { Data/ } \\
\text { Período }\end{array}$ \\
\hline Hidrografia & Cartográfica & $\begin{array}{l}\text { Caracterização Hidrogeológica dos } \\
\text { Aqüíferos do RN. }\end{array}$ & Hidroservice & 1998 \\
\hline Topografia & Cartográfica & $\begin{array}{l}\text { Carta Topográfica Folhas Natal SB } \\
25-\text {-V-C-V e São José de Mipibu } \\
\text { SB 25-Y-A-II e SB 25-Y-A-III. }\end{array}$ & SUDENE & 1983a,b,c \\
\hline $\begin{array}{l}\text { Uso e Ocupação do } \\
\text { Solo }\end{array}$ & Cartográfica & Mapa de uso e ocupação do solo. & Hidroservice & 1998 \\
\hline $\begin{array}{l}\text { Formações } \\
\text { geológicas }\end{array}$ & Cartográfica & $\begin{array}{l}\text { Caracterização Hidrogeológica dos } \\
\text { Aqüíferos do RN. }\end{array}$ & Hidroservice & 1998 \\
\hline \multirow[t]{2}{*}{$\begin{array}{l}\text { Monitoramento } \\
\text { Hidrogeológico }\end{array}$} & Cartográfica & $\begin{array}{l}\text { Mapa potenciométrico do aqüífero } \\
\text { livre da região do Bonfim - RN. }\end{array}$ & Pereira & 2001 \\
\hline & Tabular & $\begin{array}{l}\text { Piezometria dos poços e do } \\
\text { sistema de lagoas. }\end{array}$ & SERHID & 1999-2002 \\
\hline \multirow[t]{3}{*}{$\begin{array}{l}\text { Monitoramento } \\
\text { Hidrometeorológico }\end{array}$} & Tabular & $\begin{array}{l}\text { Pluviometria diária de } \\
\text { Floresta. }\end{array}$ & EMPARN & 1992-2002 \\
\hline & & Climatologia diária de Natal. $^{*}$ & $\begin{array}{l}\text { Estação } \\
\text { Climatológica } \\
\text { da UFRN }\end{array}$ & $1992-2002$ \\
\hline & & $\begin{array}{l}\text { Climatologia mensal de } \\
\text { Parnamirim. }\end{array}$ & CATRE & 1960-1999 \\
\hline $\begin{array}{l}\text { Monitoramento } \\
\text { Operacional }\end{array}$ & Tabular & $\begin{array}{l}\text { Volumes mensais produzidos para } \\
\text { a adutora Trairi/Potengi. }{ }^{*}\end{array}$ & CAERN & $1999-2002$ \\
\hline
\end{tabular}

( ) Informações cedidas mediante solicitação por oficio.

\footnotetext{
${ }^{19}$ Informação cedida mediante solicitação por oficio.
} 


\subsubsection{Modelo Computacional}

A construção do modelo computacional de águas subterrânea da área de estudo é feita utilizando-se o Método de Elementos Analíticos (capítulo 3) adaptado a semi-planos (capítulo 4). Foram utilizados os programas GNU TimSL 0.3 estendido (seção 3.5) e o programa apresentado no capítulo anterior (seção 4.4).

Observando-se o fato de que as linhas da costa e do afloramento seguem a mesma tendência durante um trecho bastante longo, o domínio do modelo é caracterizado por duas linhas retas paralelas (Figura 5.2.1). Nesse primeiro momento, é feita uma abordagem panorâmica do aqüífero estudado, não sendo, ainda, abordada a escala da área de estudo (ver destaque feito pelas linhas tracejadas na Figura 5.2.1).

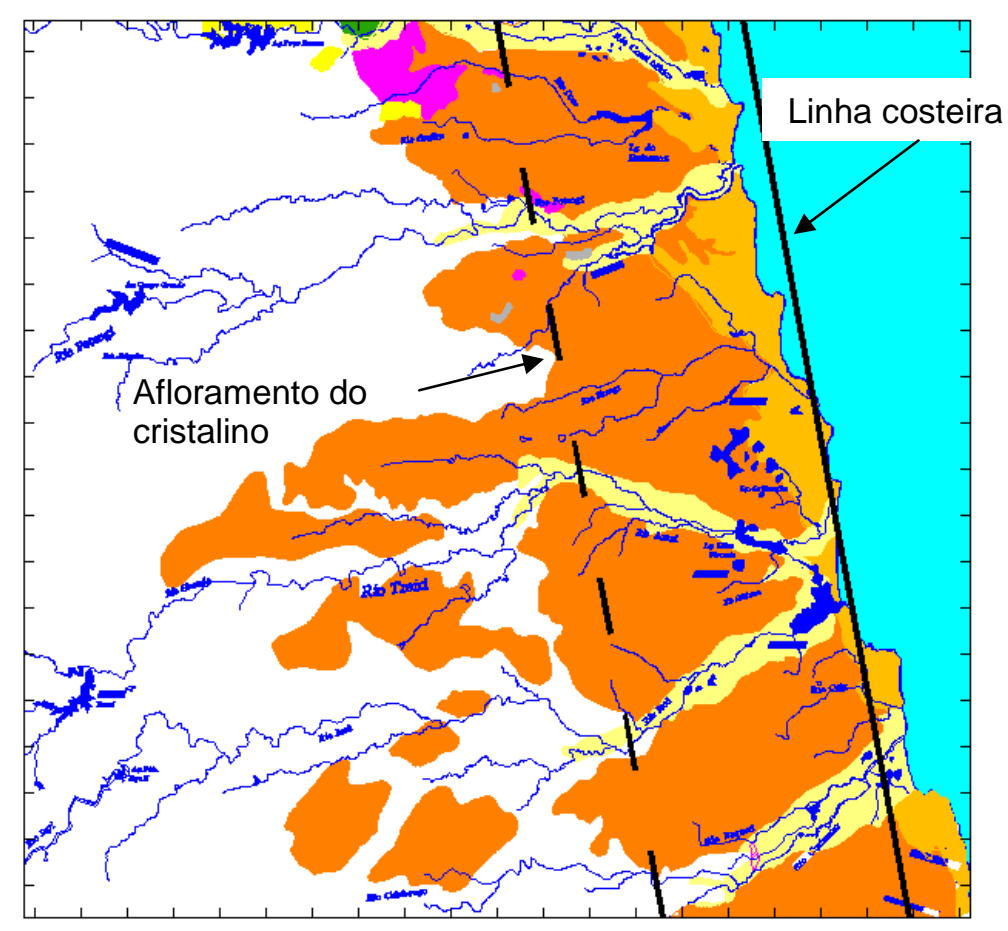

Figura 5.2.1 - Aproximação geométrica do Aqüífero Barreiras próximo à Área de Estudo

$\mathrm{Na}$ abordagem panorâmica, as propriedades do aqüífero e sua recarga são representadas uniformemente distribuídas. Pode-se observar que, nesta etapa da construção do modelo, o aqüífero é abordado de uma maneira bastante simplificada. Matematicamente, é representado apenas o efeito do escoamento devido à recarga panorâmica, expresso pela fórmula do escoamento unidimensional entre dois drenos (seção 4.3). Os parâmetros adotados nesta etapa 
da modelagem foram obtidos em estudos anteriores (e.g. Pereira, 2001 e Melo e Feitosa, 1998). A taxa de recarga, no entanto, é avaliada neste trabalho por meio da calibração da potenciometria anterior à implantação do sistema.

A representação dos efeitos produzidos na área de estudo constitui a segunda etapa da construção do modelo computacional. Nessa etapa é feita a aplicação de Elementos Analíticos ao modelo. A Formação Dunas e a hidrografia local são representadas por meio de elementos retilíneos, exceto as pequenas lagoas à leste da Lagoa do Bonfim, que são representadas por elementos dipolo e poço.

A Formação Dunas apresenta uma condutividade hidráulica média diferenciada da Formação Barreiras (e.g. Pereira, 2001 e Melo e Feitosa, 1998). Dessa forma, os contornos da Formação Dunas são representados por meio de elementos linedoublets.

A representação de riachos, lagoas e poços da região é feita utilizando-se elementos linesinks. São dispostos linesinks sobre a Lagoa do Bonfim e a hidrografia próxima à Lagoa até uma distância pragmaticamente arbitrada em $10 \mathrm{~km}$ na direção longitudinal do aqüífero. A condição de contorno utilizada nos riachos é do primeiro tipo, ou seja, cargas piezométricas conhecidas ao longo do corpo hídrico. Os valores utilizados são retirados das cotas topográficas das margens dos cursos de água. A disposição dos elementos utilizados é ilustrada na Figura 5.2.2.

Os elementos utilizados na representação da Lagoa do Bonfim, impõem ao escoamento a condição do segundo tipo. Dessa forma, o balanço hídrico da Lagoa, obtido dos dados hidrometeorológicos coletados neste trabalho (ver seção 5.2.2), é imposto sobre o escoamento. De maneira semelhante, é imposto o balanço hídrico atmosférico das demais lagoas do complexo, no entanto, os elementos utilizados são as funções dipolo e poço. Os poços de bombeamento, por sua vez, da mesma forma, impõem seu efeito sobre o modelo por meio da função poço utilizando-se valores de vazões de bombeamento conhecidas.

Foram utilizados neste modelo 576 elementos de segunda ordem. Assim, são resolvidas 1994 incógnitas correspondentes aos esforços naqueles elementos cujos parâmetros não são conhecidos a priori. Utiliza-se, ainda, três pontos de controle por elemento retilíneo, sobrespecificando os elementos e totalizando-se 2823 equações. O arquivo de entrada para as simulações feitas a seguir encontra-se no CD em anexo. 


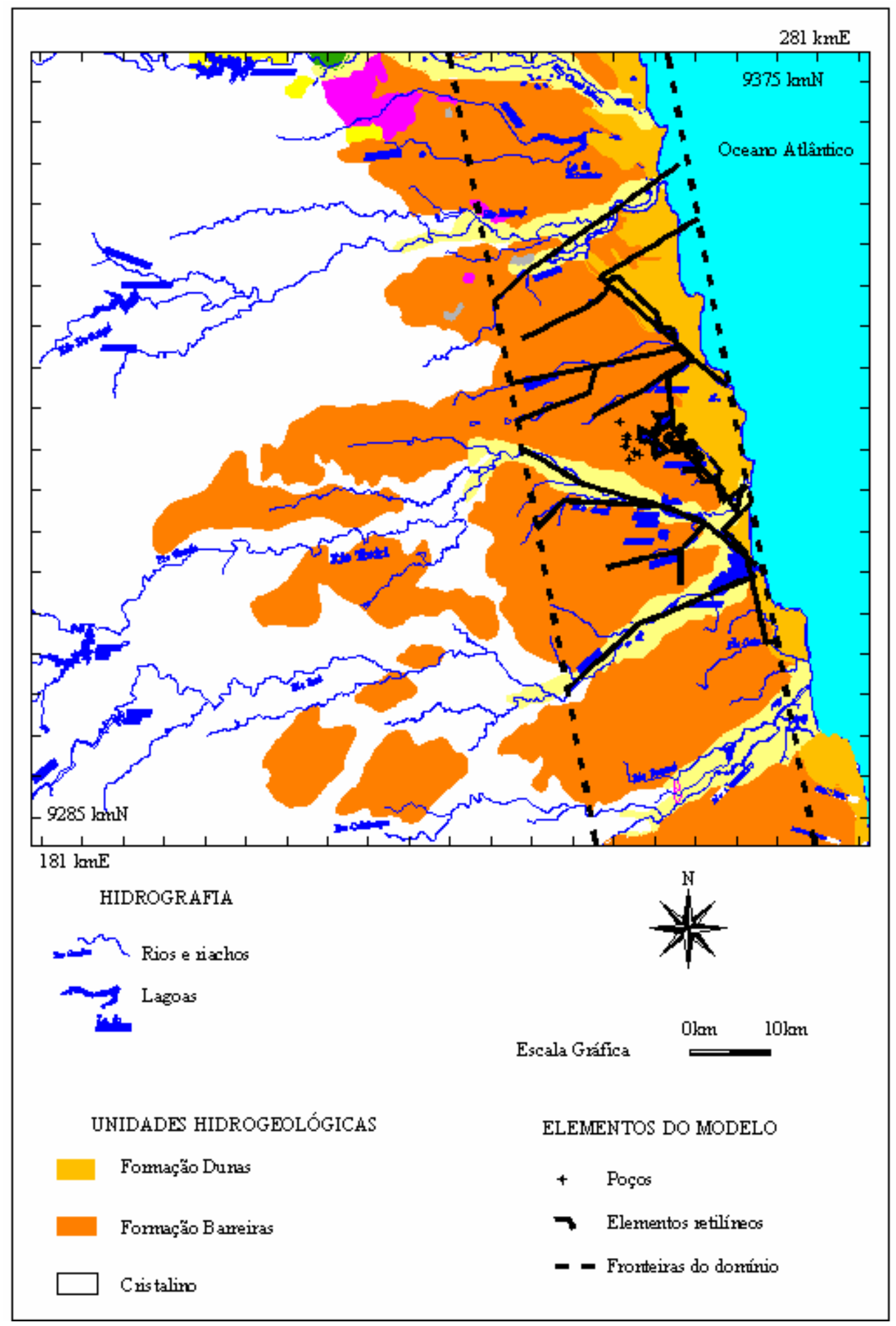

Figura 5.2.2 - Disposição de Elementos Analíticos sobre a Área de Estudo 


\subsubsection{Calibração}

O valor de recarga regional foi obtido de forma a calibrar o nível de água da Lagoa do Bonfim. Batista (2001), utilizando um modelo de balanço hídrico de reservatórios, simulou o comportamento do nível de água na Lagoa do Bonfim para a série histórica de precipitações da região. Em seu estudo foi encontrado um valor médio da cota do espelho de aproximadamente $41,65 \mathrm{~m}$, portanto, a cota a ser utilizada na calibração da recarga.

O modelo foi construído conforme descrito na seção 5.2.3 e os dados foram utilizados de acordo com os valores apresentados na Tabela 5.2.2, extraídos de trabalhos anteriores (e.g. Pereira, 2001 e Melo e Feitosa, 1998). É considerada, ainda, uma taxa de evapotranspiração potencial sobre o espelho de água das lagoas de 146,31 mm/mês. Dessa forma, a taxa de recarga foi calibrada em 24,0 mm/mês, fornecendo a cota de espelho desejada na Lagoa do Bonfim. Este valor é $25 \%$ superior ao utilizado dos trabalhos anteriores, no entanto, considerando-se a escassez de estudos acerca deste parâmetro, o valor aqui obtido pode ser utilizado como uma verificação de sua ordem de grandeza.

Tabela 5.2.2 - Valores de Parâmetros Hidrogeológicos Disponíveis na Literatura

\begin{tabular}{c|c|c|c|c}
\hline Meio & $\begin{array}{c}\text { Condutividade } \\
(\mathrm{m} / \mathrm{mês})\end{array}$ & $\begin{array}{c}\text { Cota da base } \\
(\mathrm{m})\end{array}$ & $\begin{array}{c}\text { Porosidade } \\
(\%)\end{array}$ & $\begin{array}{c}\text { Taxa de Recarga } \\
(\mathrm{mm} / \mathrm{mês})\end{array}$ \\
\hline Formação Barreiras & 300,0 & $-20,0$ & 10,0 & 18,0 \\
\hline Formação Dunas & 123,0 & $-20,0$ & 3,0 & 18,0 \\
\hline
\end{tabular}

Embora potenciometrias verificadas em campo configurem um instante particular de um escoamento em regime não-permanente, os dados hidrometeorológicos registrados durante o estudo de Pereira (2001) são próximos dos valores médios. Assim, a potenciometria obtida é confrontada com a potenciometria de Pereira (2001) na Figura 5.2.3.

Muito embora, a resposta obtida do modelo não reproduza a superfície potenciométrica em toda a área de estudo, a potenciometria simulada reproduz o sistema de fluxo identificado em campo. Regiões onde a simulação apresenta diferenças a partir do comportamento observado são representativas de heterogeneidades do meio não caracterizadas nas investigações anteriores e, portanto, não declaradas ao modelo. 


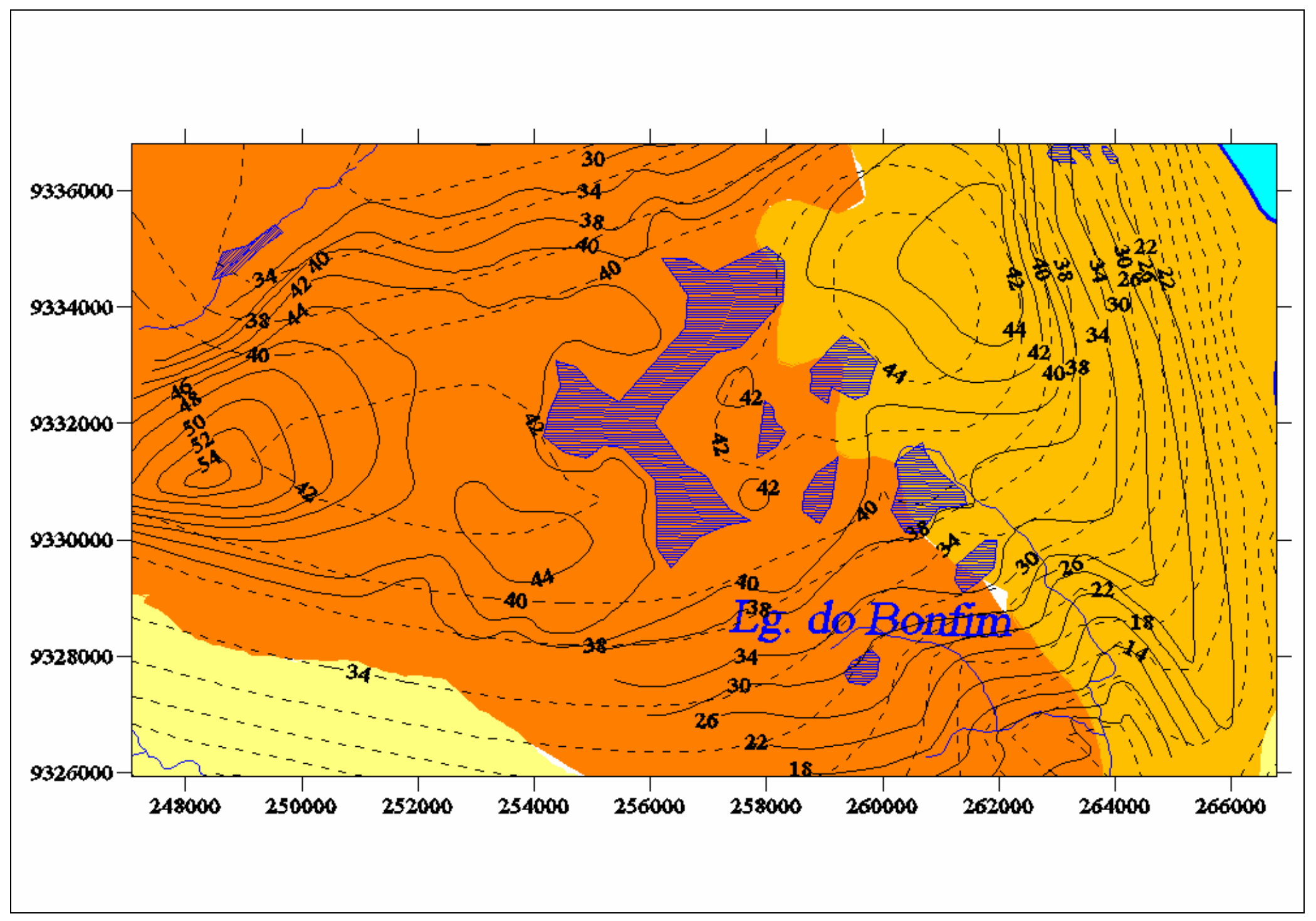

Figura 5.2.3 - Comparação entre a Potenciometria Simulada (linha tracejada) e a Monitorada (linha contínua) na Área de Estudo 


\subsubsection{Resultados e Discussões}

Esta seção encontra-se dividida em duas partes. Na primeira, a mitigação do impacto da captação sobre a Lagoa do Bonfim por meio de poços suplementares é avaliada. Em seguida, a ocorrência de um evento hidrológico extremo é discutida a fim de se identificar o impacto do projeto sob condições naturais extremas.

\subsubsection{Avaliação da Mitigação do Impacto sobre a Lagoa do Bonfim}

Visando uma avaliação da influência da implantação da primeira bateria de poços auxiliares do Sistema Adutor Agreste/Trairi/Potengi sobre o espelho de água da Lagoa do Bonfim, são considerados dois cenários. No primeiro cenário é simulada a potenciometria da região considerando-se a captação apenas na Lagoa. No segundo cenário é introduzido o bombeamento dos poços (ver Tabela 5.2.3) e utilizado o valor médio dos volumes bombeados na Lagoa após o início da operação dos poços. Assim, no primeiro cenário, é utilizado o volume médio produzido na Lagoa durante Nov/98 a Mai/00 ( $\left.\bar{Q}=500.000 \mathrm{~m}^{3} / \mathrm{mês}\right)$ e no segundo cenário, o volume produzido na Lagoa, entre Jul/00 e Mar/02 ( $\bar{Q}=281.016,0$ $\mathrm{m}^{3} /$ mês). Os volumes médios bombeados em cada poço, durante o mesmo período, são apresentados na Tabela 5.2.3.

Tabela 5.2.3 - Localização e Vazões de Bombeamento dos Poços da Adutora

\begin{tabular}{c|c|c|c}
\hline Poços & $\begin{array}{c}\text { UTM E } \\
(\mathrm{m})\end{array}$ & $\begin{array}{c}\text { UTM N } \\
(\mathrm{m})\end{array}$ & $\begin{array}{c}\text { Vazões } \\
\left(\mathrm{m}^{3} / \mathrm{mês}\right)\end{array}$ \\
\hline 1 & 254184,2 & 9329942,4 & 48200,0 \\
\hline 2 & 253673,7 & 9330168,1 & 44700,0 \\
\hline 3 & 252536,4 & 9330893,8 & 39600,0 \\
\hline 4 & 252707,2 & 9331760,6 & 23500,0 \\
\hline 5 & 252757,14 & 9332137,4 & 59700,0 \\
\hline 6 & 251867,75 & 9333917,16 & 25000,0 \\
\hline 7 & 253057,4 & 9329284,2 & 42000,0 \\
\hline
\end{tabular}




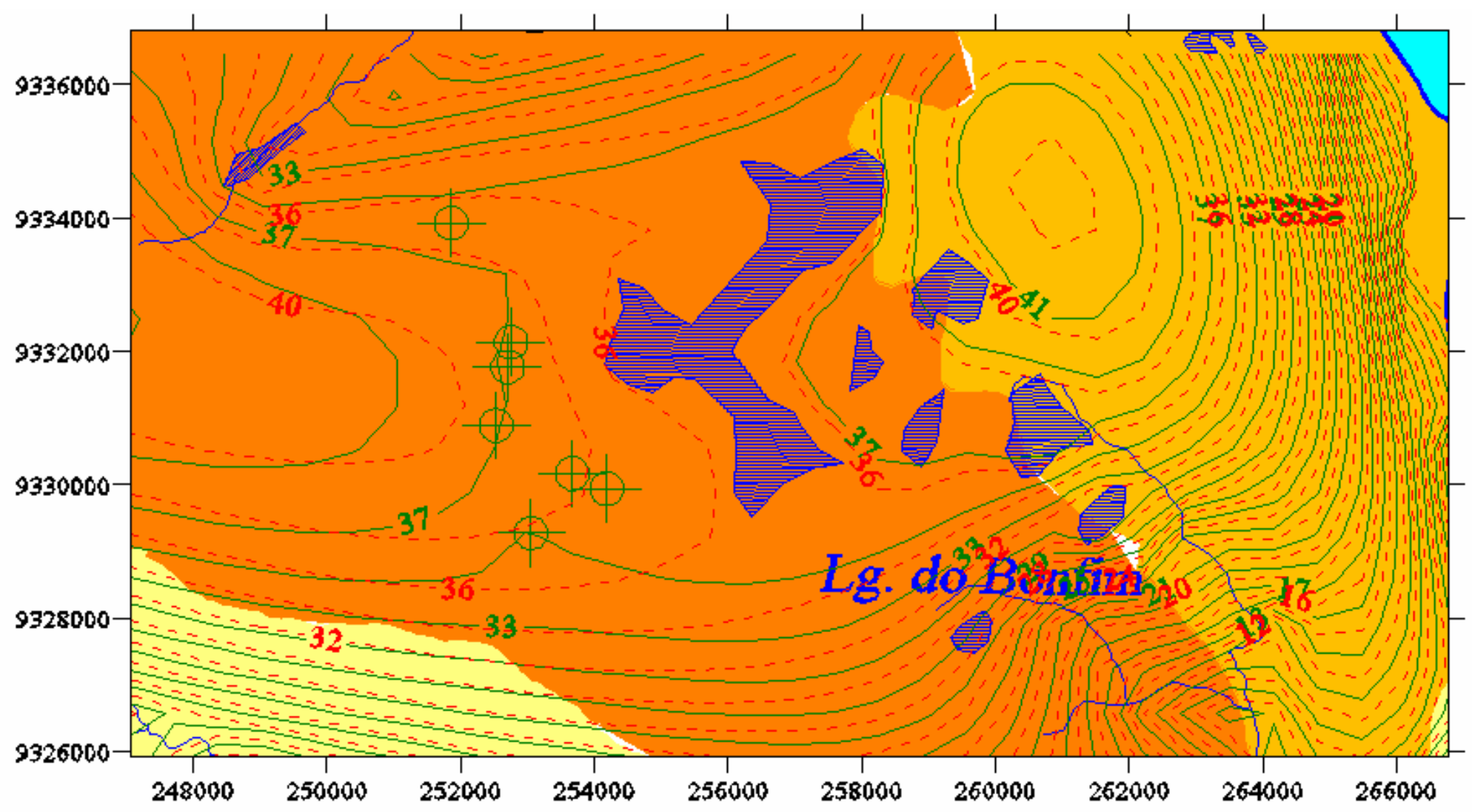

Figura 5.2.4 - Comparação entre a Potenciometria do Cenários Estacionários da Captação apenas na Lagoa (linhas tracejadas) e da Captação Mista (linhas contínuas). 
A distribuição obtida em ambos cenários é ilustrada na Figura 5.2.4. Em ambos cenários são encontrados valores ainda não confirmados pelo monitoramento atual da Lagoa. Somente uma calibração das porosidades das Dunas e das Barreiras permitiria uma avaliação do tempo necessário para a estabilização da potenciometria após a implantação do projeto, promovendo-se assim a verificação do modelo. Entretanto, embora a avaliação de cenários em regime estacionário não necessite de dados de porosidade, o resultado pode ser utilizado como avaliação tendo em vista a redução média do espelho de água da Lagoa observada de aproximadamente dois metros em seis anos de operação. Devido à extensão do escoamento, o tempo necessário para o estabelecimento do escoamento originado pela captação pode ser ainda maior.

Na Figura 5.2.4, apresenta-se, a simulação da potenciometria estacionária obtida da captação simples da Lagoa (linhas tracejadas) e da captação mista (linhas contínuas), introduzindo-se o bombeamento dos poços. Em ambos cenários, observa-se cotas potenciométricas inferiores àquelas simuladas com sem a presença da captação (Figura 5.2.3, linhas tracejadas). No primeiro cenário, representando a configuração original do projeto (captação simples), obtém-se uma diferença máxima de $6,18 \mathrm{~m}$, localizada sobre o espelho de água da Lagoa. No segundo cenário, configurando a ação mitigadora prevista no projeto (suplementação da captação por meio de poços), obtém-se uma leve recuperação do espelho de água de $0,82 \mathrm{~m}$ após atingir novamente o regime estacionário.

\subsubsection{Avaliação do Impacto Devido à Ausência de Recarga Direta na Região}

Considerando a ocorrência de secas na região, é avaliado agora o impacto da captação sob um cenário onde não ocorre recarga do aqüífero. A seca avaliada refere-se a um período de dois meses onde é simulada a ausência total de precipitação na área de estudo. Assim, a partir do regime estacionário do bombeamento do Sistema Lacustre, é simulada a ocorrência da seca. Embora a estação seca da região seja compreendida durante todo verão e início do outono (em média quatro meses), o período compreendido pela simulação encontra restrições devido ao grande tempo de processamento demandado pela formulação transiente do Método de Elementos Analíticos (ver seção 4.5).

Os resultados, ilustrados na Figura 5.2.6, permitem visualizar mudanças na potenciometria devido ao processo de reconfiguração do escoamento no instante que a seca atinge dois meses de duração. Nesse instante, observa-se um acréscimo no rebaixamento permanente do espelho de água da Lagoa o Bonfim de aproximadamente 0,5 $\mathrm{m}$. Com a 
continuação do evento, valores ainda maiores poderão ser encontrados, entretanto, o resultado apresenta-se com a mesma ordem de grandeza encontrada em monitoramentos limnimétricos entre janeiro e março de 2001 (ver Figura 5.2.5). As discrepâncias entre o resultado e a redução observada podem ser devidas à ocorrência de recargas ainda no início de janeiro.

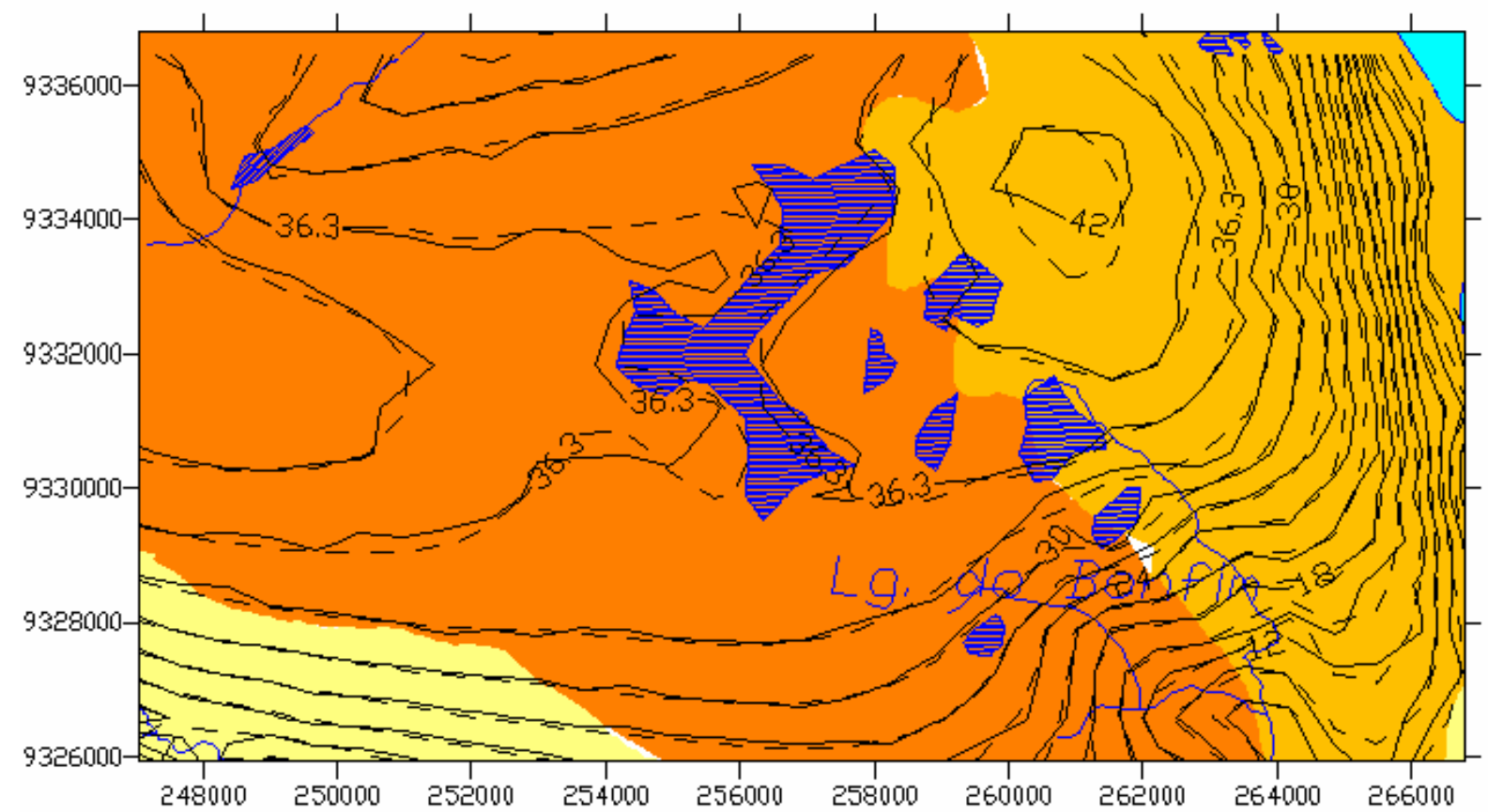

Figura 5.2.5 - Potenciometria Comparativa do Cenário Permanente (linhas tracejadas) e do Cenário Transiente Devido à Seca (linhas contínuas).

Período Jan/2001 a Mar/2001

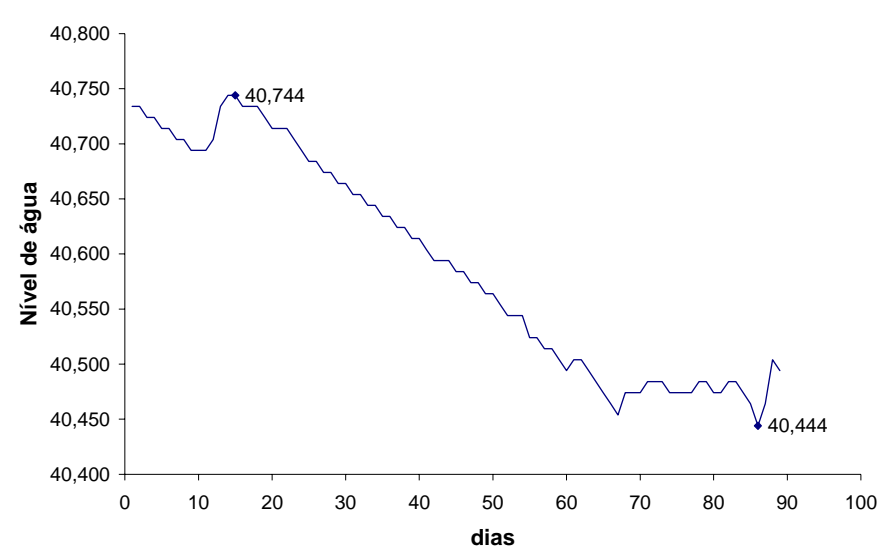

Figura 5.2.6 - Redução do Nível do Espelho de Água da Lagoa do Bonfim entre Janeiro e Março de 2001 (Fonte: SERHID) 


\section{Conclusões}

A representação de fronteiras de Aqüíferos Longos por meio de Elementos Analíticos pode introduzir dúvidas quanto ao posicionamento dos mesmos ou, ainda, efeitos de borda à simulação. Neste sentido, a hipótese de Aqüíferos Longos introduz realismo à modelagem uma vez que os contornos externos do aqüífero são mapeados convenientemente pelo eixo de um semi-plano.

O programa GNU TimSL 0.3 (Bakker et al., 2002), onde elementos estacionários e transientes de primeira ordem já haviam sido implementados, foi estendido de modo a compreender as correções e melhorias obtidas neste trabalho. Foram implementadas as expressões dos elementos estacionários de segunda ordem de Strack (1989) e as imposições de continuidade e suavidade dos polinômios obtidas na seção 3.2 .4 obtendo-se uma suavisação vantajosa para a representação do escoamento. As expressões de elementos transientes utilizadas na versão original do programa produziam resultados insatisfatórios para a utilização desses elementos em modelos. Os comportamentos indesejados foram identificados e corrigidos, obtendo-se, então, resultados mais regulares para o comportamento dos elementos. Além disso, foram formulados novos elementos transientes utilizando-se distribuição de esforços de segunda ordem, verificando-se maiores suavidade e precisão em relação aos elementos de primeira ordem.

O acoplamento do Método de Imagens para elementos estacionários e transientes é facilmente obtido (capítulo 4). O mapeamento de elementos mediante transformações de Schwarz-Christoffel, no entanto, exigem a reformulação dos mesmos. Os elementos estacionários estão reformulados para o mapeamento de Aqüífero Longos caracterizados por apenas duas retas. As integrais utilizadas, neste caso, necessitam ser resolvidas de forma híbrida, parte analiticamente e parte numericamente mediante quadraturas. A reformulação obtida não é válida para elementos transientes, assim, a utilização estes elementos em Aqüíferos Longos pode ser feita, mediante o Método de Imagens visando a representação das fronteiras do aqüífero. Neste caso o uso do Método de Imagens é feito assumindo-se um truncamento no número de imagens, introduzindo-se erros na simulação em relação às condições especificadas na fronteira do domínio à medida que os transitórios são propagados. A metodologia de Aqüíferos Longos também foi adicionada ao programa Tim ${ }^{\mathrm{SL}}$ estendendose a aplicabilidade do programa para a utilização de elementos em aqüíferos semi-infinitos. 
O modelo de Elementos Analíticos baseado na hipótese de Aqüífero Longo, apresentado no capítulo 5, por fim, obteve bons resultados de calibração em relação à potenciometria de uma região do Aqüífero Barreiras no Estado do Rio Grande do Norte. Ainda no mesmo capítulo o modelo é utilizado na avaliação de impactos permanentes (em regime estacionário) do Sistema Adutor Agreste/Trairi/Potengi sobre a Lagoa do Bonfim e impactos transientes (simulação da ausência de precipitações na região). Os resultados da calibração apontam uma recarga média de $24 \mathrm{~mm} /$ mês e condutividades hidráulicas de 10m/dia na Formação Barreiras e 4,1m/dia na Formação Dunas. A avaliação de impactos fornece uma redução permanente de 5,36m sobre o nível de água da Lagoa do Bonfim. Embora a redução ainda não tenha sido verificada in loco, É realizada, ainda, uma simulação da potenciometria devido à operação do Sistema sujeita a dois meses na ausência de recargas diretas. Durante a simulação do cenário de seca, o nível de água da Lagoa sofre um decréscimo de aproximadamente $0,5 \mathrm{~m}$. O período compreendido pela simulação sofre restrições neste trabalho devido ao grande tempo computacional exigido pela formulação dos elementos transientes. 


\section{Referências Bibliográficas}

Anderson, E. I. (2000) The method of images for leaky boundaries. Adv. Water Resour., 23, pp461-74.

Bakker M.; Kelson, V.; Zaadnoordijk, W.J. (2002) An Object-Oriented Analytic Element Program Written in Python In: http://www.engr.uga.edu/ mbakker/timsingle.html (acessado em dezembro de 2005).

Bakker, M. e Anderson, E. I. (2004) Steady Flow to a Well Near a Stream with a Leaky Bed. Ground Water. v41, n6, pp833-840.

Bakker, M.; Strack, O.D.L. (2003) Analytic Elements for Multiaquifer Flow. Journal Of Hydrology. v271 pp119-129.

Batista, J.A.N. (2001) Caracterização da Reserva Hídrica da Lagoa do Bonfim Frente à Captação da Adutora Monsenhor Expedito - RN. 143p. Dissertação de Mestrado. Universidade Federal do Rio Grande do Norte. Natal.

Batista, J.A.N., Schulz, H.E., Wendand, E.C. (2004) Método de Imagens para Representação de Contornos Regionais em Águas Subterrâneas. In: Proceedings of XXV Iberian Latin American Congress of Computational Methods for Engineering. Recife. CDROM.

Bear, J. (1972) Dynamics of Fluids in Porous Media, Elsevier Science, Nova York.

Bischoff, H. (1981) An integral equation method to solve three dimensional confined flow to drainage systems. Appl. Math. Modelling. v5. pp 399-404.

Carslaw, H.S. e Jaeger, J.C. (1959) Conduction of heat in solids, Oxford University Press, Oxford.

De Josselin de Jong, G. (1960) Singularity distributions for the analysis of multiple fluid flow through porous media, J. Geophys. Res. n.35, pp. 3739-3758.

De Lange, W. J. (1999) A Cauchy boundary condition for the lumped interaction between an arbitrary number of surface waters and a regional aquifer. J. Hydrology. v226, n34, pp 250-261.

Duschek, A. e Hochrainer, A. (1954) Grundzüge der Tensorrechnung in Analytischer Darstellung. In: Tensoranalysis vol. 2, Springer-Verlag, New York, 1954.

Fetter, C.W. (1994) Applyed Hydrologeology, Prentice-Hall, Englewood Cliffs.

Free Software Foundation (1999) GNU Lesser General Public License. In: http://www.fsf.org/licensing/licenses/lgpl.html (acessado em dezembro de 2005). 
Fundação Getúlio Vargas (1998). Plano Nacional de Recursos Hídricos. Brasília.

Furman, A. e Neuman, S.P. (2003). Laplace-transform analytic element solution of transient flow in porous media. Advances in Water Res. 26(12), 1229-1237.

Furman, A. e Neuman, S.P. (2004). Laplace transform analytic element method for transient flow problems. In Proceedings of the 14th Computational Methods in Water Resources Conference (CMWR), June, Chapel Hill.

Georghitza, St. I. (1969) On the Plane Steady Flow of Water through Inhomogeneous Porous Media. In: 1st Symp. The Fundamentals of Transport Phenomena in Porous Media. IAHR, Haifa.

Haitjema, H. M. (1985) Modeling three-dimensional flow in confined aquifers by superposition of both two- and three-dimensional analytic functions, Water Resour. Res., v.21, n. 10, pp 1557-1566.

Haitjema, H. M. (1991) An analytic element model for transient axi-symmetric interface flow, J. Hydrology, n129, pp. 215-244.

Hemker, C.J. (1984) Steady Groundwater Flow In Leaky Multiple-Aquifer Systems. J. Hydrology. v72, pp355-374.

Hidroservice Engenharia LTDA (1998) Plano Estadual de Recursos Hídricos do Rio Grande do Norte. Relatório Síntese (HE-1358-R30-1198). 265p. Secretaria de Estado dos Recursos Hídricos do Rio Grande do Norte. Natal.

Hubbert, M. K. (1956) Darcy's law and the field equation of the flow of underground fluids, Translactions of American Institut of Mine and Metal Engineering. n.207 pp. 222-239.

Hubbert, M.K. (1969) The theory of ground-water motion and related papers. Hafner. New York.

Janković, I. (1997) High Order Analytic Elements in Modeling Groundwater Flow. Tese de Doutorado. Universidade de Minnesota.

Keller, J.B. (1953) The scope of the Image Method, Comm. on Phys. and App. Math. v4, pp505-512.

Kellogg, O.D. (1953) Foundation of Potential Theory, Dover Publishing, New York.

Kobe, D.H. (1986) Helmholtz's theorem revisited, Am. J. Phys. v54, p552-554.

Luther, K.H e Haitjema, H.M. (2000) Approximate analytic solutions to 3D unconfined groundwater flow within regional 2D models, Journal of Hydrology, n. 229, pp. 101-117.

Luther, K.H. (1998) Analytic Solutions to Three-dimensional Unconfined Groundwater Flow Near Wells. Tese (PhD). Universidade de Minnesota. 
Manuel Filho, J. e Castro, V.L.L. (2002). Avaliação e Explotação do Aqüífero DunasBarreiras na Região do Subsistema Lacustre do Bonfim - Rn. In: CD dos Anais do XII Congresso Brasileiro de Águas Subterrâneas. Florianópolis.

Melo, J.G. e Feitosa, E.C. (1998). Pesquisa Hidrogeológica do Complexo Lagunar do Bonfim. SERHID. Natal.

Mitchell-Bruker, S. e Haitjema, H.M. (1996) Modeling Steady State Conjunctive Groundwater and Surface Water Flow with Analytic Elements, Water Resourc. Res., Vol. 32, No. 9, pp $2725-2732$.

Muskhelishvili, N.I. (1953) Some basic problems of the mathematical theory of elasticity. Wolters-Noordhoff, Groningen.

Pereira, R. (2001). Caracterização Hidrogeológica do Sistema Lacustre Bonfim - RN. Tese de Doutorado. Universidade Federal do Rio de Janeiro. Rio de Janeiro.

Pereira, R., Chiavenato, M.C., Freitas, J., Reis, L.M.M., Lima, M.M. (1996). Carta Geotécnica do Município de Nísia Floresta - RN. In: Simpósio Brasileiro de Cartogragia Geotécnica. Boletim n 2, p233-239. São Carlos.

Pereira, R., Guimarães Júnior, J.A., Silva Júnior, G.C. (2003). Estado da Arte da Bacia Hidrogeológica do Sistema Lacustre Bonfim, RN - Nordeste do Brasil. Rev. Águas Subterrâneas. n17, pp 41-47.

Pereira, R., Guimarães Júnior, J.A., Silva Júnior, G.C., Mattos, A. (2002). Avaliação do Impacto da Captação de Água na Lagoa do Bonfim, RN - Brasil. Rev. Águas Subterrâneas. n 16, pp 75-84.

Steward, D. R. (2001). A vector potential for a partly penetrating well and flux in an approximate method of images. Proceedings of The Royal Society of London, Series A 457(2013), 2093-2111.

Strack, O. D. L. (1981). Flow in aquifers with clay laminae: 1. The comprehensive potential. Water Resour. Res. 17 (4), 985-992.

Strack, O. D. L. (1981). Flow in aquifers with clay laminae: 2. Exact solutions. Water Resour. Res. 17 (4), 993-1004.

Strack, O. D. L. (1989) Groundwater mechanics, Prentice-Hall, Englewood Cliffs.

Strack, O.D.L. e Haitjema, H.M., (1981) Modeling double aquifer flow using a comprehensive potential and distributed singularities, 2. Solution for inhomogeneous permeabilities, Water Resour. Res. v17, n5, pp. 1551-1560.

Stroud, A.H. e Secrest, D. (1966) Gaussian quadrature formulas. Prentice-Hall. Englewood Cliffs. 
Superintendência de Desenvolvimento do Nordeste (1983). Mapa Topográfico Folha Natal (SB 25-V-C-V). Recife.

Superintendência de Desenvolvimento do Nordeste (1983). Mapa Topográfico Folha São José de Mipibu (SB 25-Y-A-II). Recife.

Superintendência de Desenvolvimento do Nordeste (1983). Mapa Topográfico Folha São José de Mipibu (SB 25-Y-A-III). Recife.

Villela, S.M. e Mattos, A. (1975) Hidrologia Aplicada. McGraw-Hill do Brasil. São Paulo.

Zaadnoordijk, W.J. (1988) Analytic Elements for Transient Groundwater Flow. Tese de Doutorado. Universidade de Minnesota.

Zaadnoordijk, W.J. e Strack, O.D.L. (1993) Area sinks in the analytic element method for transient groundwater flow, Water Resour. Res. v29, n12, pp4121-4129. 


\section{Apêndice A}

\section{DETERMINAÇÃO DO POTENCIAL COMPLEXO PARA ELEMENTOS ESTACIONÁRIOS}

\section{A.1 Elementos Circulares}

Os elementos circulares foram discutidos e utilizados neste trabalho conforme Strack (1981) a partir de funções de dipolo posicionadas no centro de cada elemento desejado. Embora a função dipolo tenha sido discutida apenas para a aplicação de um lago circular, a função pode ser empregada semelhantemente na representação de regiões circulares de diferentes condutividades especificando-se a condição de contorno da Eq. 3.4.15. As aplicações de regiões heterogêneas circulares em escoamentos subterrâneos são diversas e têm crescido significativamente na caracterização de aqüíferos com grau de heterogeneidade elevado (e.g. Dagan et al. 2003, Fiori et al. 2003, I. Janković et al. 2003). Elementos circulares podem ser apresentados a partir de uma distribuição de densidade assim como nos elementos unifilares em Salisbury (1992 apud Janković,1997, pág. 56) ${ }^{20}$ como:

$$
\Omega= \begin{cases}\sum_{i=0}^{\infty} \lambda_{i} Z^{i} & , \text { para }|Z|<1 \\ \sum_{i=1}^{\infty} \bar{\lambda}_{i} Z^{-i} & , \\ \text { para }|Z| \geq 1\end{cases}
$$

onde $\lambda_{i}$ é o número complexo que denota intensidade e orientação do dipolo e $Z_{i}$ é a coordenada adimensional definida em função da coordenada do centro $\mathrm{Z}$ e do raio $R$,como:

$$
Z=\left(Z-{ }^{c}\right) / R
$$

\section{A.2 Elementos unifilares}

${ }^{20}$ Salisbury, M.L. (1992) Control equation formulation for circular inhomogeneities in the analytical element method. Dissertação (Mestrado). University of Minnesota. 


\section{A.2.1 Linedipole e linedoublet}

A solução da integral linedipole é dada no capítulo 2 (Eq. 2.3.16) por:

$$
\Omega=-\frac{\mu(Z)}{2 \pi} \ln \frac{Z-1}{Z+1}+q(Z)
$$

onde $\mu(Z)$ é expresso na forma de Eq. 2.3.14:

$$
\mu(Z)=\sum_{j=0}^{n} a_{j} Z^{j}
$$

sendo $a_{i}$ é um coeficiente real e $p(Z)$ um polinômio de ordem $n-1$ escrito na forma Eq. 2.3.15:

$$
p(Z)=\sum_{m=0}^{n-1} b_{m} Z^{m}
$$

Uma linedoublet tem a mesma definição que uma linedipole a menos da orientação do dipolo que é girado de um ângulo $\pi / 2(=\arg (i))$, portanto:

$$
\Omega=-\frac{\lambda(Z)}{2 \pi i} \ln \frac{Z-1}{Z+1}+i p(Z)
$$

Os termos $p$ e $\lambda$ podem ser determinados mais uma vez sujeitos às condições prescritas na seção 2.3.2.

\section{A.2.2 Linesink}

No capítulo 2, apresenta-se a definição de uma linha poço pela expressão Eq. 2.3.1 como: 


$$
\Omega=\int_{\substack{1 \\ z}}^{2} \frac{\sigma}{2 \pi} \ln (z-\delta) d \xi
$$

A integração por partes da expressão Eq. 2.3.10

$$
\Omega=-\frac{1}{2 \pi} \int_{\substack{1 \\ z}}^{\substack{z \\ z-\delta}} d \delta=\frac{1}{2 \pi} \int_{\substack{1 \\ z}}^{2} \mu d \ln (z-\delta)
$$

conduz a:

$$
\Omega=\frac{1}{2 \pi}\left[\mu\left(z^{2}\right) \ln \left(z-z^{2}\right)-\mu\left(z^{1}\right) \ln \left(z-z^{1}\right)\right]-\frac{1}{2 \pi} \int_{\substack{1 \\ z}}^{z} \frac{d \mu}{d \delta} \ln (z-\delta) d \delta
$$

Utilizando-se a definição Eq. 2.3.2:

$$
\frac{d \mu}{d \delta}=\frac{d \mu}{d \xi} e^{-i \alpha}
$$

Observando-se que $\mu=\Psi^{+}-\Psi^{-}$ao longo do elemento e que

$$
\partial\left(\Psi^{+}-\Psi^{-}\right) / \partial \xi=-\sigma=d \mu / d \xi
$$

obtém-se na expressão do linedipole um termo idêntico à definição Eq. 2.3.1:

$$
\Omega=\frac{1}{2 \pi}\left[\mu\left(z^{2}\right) \ln \left(z^{2}-z^{2}\right)-\mu\left(z^{1}\right) \ln \left(z^{-}-z^{1}\right)\right]-\frac{1}{2 \pi} \int_{\substack{z \\ z}}^{2} \sigma \ln (z-\delta) d \xi
$$

de forma que uma linesink pode também ser definida pela expressão: 


$$
\Omega_{l s}=-\frac{1}{2 \pi} \int_{\substack{1 \\ z}}^{2} \frac{\mu}{z-\delta} d \delta-\frac{1}{2 \pi}\left[\mu\left(z^{2}\right) \ln \left(z-z^{2}\right)-\mu\left(z^{1}\right) \ln (z-z)\right]
$$

A expressão acima pode ser utilizada com vantagem sobre a definição Eq. 2.3.1 face ao comportamento do ramo. Considerando-se uma cadeia formada por elementos linesink, ocorrerá ramo no prolongamento de cada elemento em direção a $-\infty$. O ramo de uma cadeia de linesinks escritos de acordo com a definição Eq. A.12 combina o ramo de cada dipolo, notado apenas sobre cada elemento, mais o ramo do poço localizado no primeiro ponto da cadeia. Como mencionado na seção 2.3.1, o ramo pode ser utilizado como uma avaliação do aporte de vazão no elemento. No caso de elementos modelados segundo a definição Eq. A.12, o ramo fornece o aporte para toda a cadeia de elementos (e.g. rios e lagos) e ser utilizado, por exemplo, como uma condição de contorno.

\section{A.2.3 Areasink}

As descontinuidades de potencial $\Delta \Phi$ e descarga $\Delta Q$ são eliminadas para uma areasink somando-se as correções para ambas descontinuidades dadas respectivamente pelas funções linedoublet e linesink (ver Eq. 3.2.20 e Eq. 2.3.1) na forma de:

$$
\stackrel{b}{\Omega}=-\frac{1}{2 \pi i} \oint_{C} \frac{\lambda^{b}(\delta)}{Z-\delta} d \delta+\frac{1}{2 \pi} \oint_{C}{ }^{b} \sigma \ln (z-\delta) d s
$$

Utilizando-se a relação Eq. A.9 e integração por partes para a segunda integral, resulta:

$$
\stackrel{b}{\Omega}=-\frac{1}{2 \pi i} \oint_{C} \frac{{ }^{b}(\delta)}{z-\delta} d \delta-\frac{1}{2 \pi} \oint_{C} \frac{\stackrel{b}{\mu}(\delta)}{z-\delta} d \delta-\left.\frac{\stackrel{b}{\mu}}{2 \pi} \ln (z-\delta)\right|_{C}
$$

$$
\begin{aligned}
& \text { A função } \stackrel{b}{\mu} \text { define o ramo ao longo do contorno } C \text {, logo: } \\
& \oint_{C}^{b} \sigma d s=-\oint_{C} \frac{d \mu}{d s} d s=\stackrel{b}{Q}
\end{aligned}
$$


Assim, a expressão do areasink é definida como:

$$
\stackrel{b}{\Omega}=-\frac{1}{2 \pi i} \oint_{C} \frac{\lambda^{b}(\delta)}{Z-\delta} d \delta-\frac{1}{2 \pi} \oint_{C} \frac{\stackrel{b}{\mu}(\delta)}{Z-\delta} d \delta+\frac{\stackrel{b}{Q}}{2 \pi} \ln \left(z-z_{1}\right)
$$

Reunindo-se as duas primeiras integrais da expressão Eq. A.16 em uma apenas, tem-se:

$$
-\frac{1}{2 \pi i} \oint_{C} \frac{\stackrel{b}{\lambda}(\delta)+i^{b} \mu(\delta)}{Z-\delta} d \delta=-\frac{1}{2 \pi i} \sum_{j=1}^{n}[\underset{j}{b}(\delta)+i \underset{j}{i} \mu(\delta)] \ln \frac{\stackrel{Z}{Z}_{j}-1}{Z+1}+\underset{j}{P}(\stackrel{j}{Z})
$$

e o potencial torna-se:

$$
\stackrel{b}{\Omega}=-\frac{1}{2 \pi i} \sum_{j=1}^{n}\left\{[\underset{j}{b}(\delta)+\underset{j}{i} \underset{j}{b}(\delta)] \ln \frac{Z^{j}-1}{Z+1}+\underset{j}{P}(Z)\right\}+\frac{\stackrel{b}{Q}^{j}}{2 \pi} \ln \left(z-Z_{1}\right)
$$

Observe que a expressão Eq. A.18 contém $n$ mudanças de coordenadas do tipo $Z^{j}$. Strack (1989) reescreve o potencial $\Omega^{b}$ de forma que apenas uma mudança de coordenadas $(\zeta)$ torna-se necessária. Substituindo-se em Eq. A.18 as seguintes expressões:

$$
Z_{j}+1=\frac{\zeta-\zeta_{j}}{\frac{1}{2}\left(\zeta_{j+1}-\zeta_{j}\right)}, Z_{j}-1=\frac{\zeta-\zeta_{j+1}}{\frac{1}{2}\left(\zeta_{j+1}-\zeta_{j}\right)} \text { e } Z_{j}^{2}-1=\frac{\left(\zeta-\zeta_{j}\right)\left(\zeta-\zeta_{j+1}\right)}{\frac{1}{2}\left(\zeta_{j+1}-\zeta_{j}\right)^{2}}
$$

o potencial $\stackrel{b}{\Omega}$ é encontrado como:

$$
\Omega_{j}^{b}=\frac{\gamma}{2 \pi i}\left[{ }_{j}^{b} \ln \frac{\zeta-\zeta_{j+1}}{\zeta-\zeta_{j}}+p_{j}(\zeta)\right]
$$

$P(Z)$ é determinado reescrevendo-se Eq. 3.2.59 como:

$$
\left.\underset{j}{\chi}=\frac{1}{\gamma} \underset{j}{\stackrel{b}{\lambda}} \underset{j}{\stackrel{b}{\mu}}\right)=\underset{j}{c_{2}} \zeta^{2}+\underset{j}{c_{1}} \zeta+\underset{j}{c_{0}}
$$


Substituindo-se as relações Eq. A.19 em Eq. 3.2.60, tem-se:

$$
\begin{aligned}
& c_{j}=-\frac{1}{2} \frac{\xi_{j+1}-\xi_{j}}{\zeta_{j+1}-\zeta_{j}}, c_{j}=\frac{\xi_{j+1}-\xi_{j}}{\zeta_{j+1}-\zeta_{j}} \zeta_{j}-\xi_{j} \\
& c_{0}=-\frac{1}{2} \frac{\xi_{j+1}-\xi_{j}}{\zeta_{j+1}-\zeta_{j}} \zeta_{j} \zeta_{j+1}-\frac{1}{2}\left(\xi_{j}+\xi_{j}\right) \zeta_{j}-\frac{1}{2} \xi_{j}^{2}+i v_{j}
\end{aligned}
$$

e fazendo $\stackrel{b}{\Omega} \rightarrow \zeta^{-1}$ quando $\zeta \rightarrow \infty$ na equação Eq. A.20, pode-se escrever:

$$
\ln \frac{\zeta-\zeta_{j+1}}{\zeta-\zeta_{j}}=\ln \frac{1-\zeta_{j+1} / \zeta}{1-\zeta_{j} / \zeta}=\ln \left(1-\frac{\zeta_{j+1}}{\zeta}\right)-\ln \left(1-\frac{\zeta_{j}}{\zeta}\right)
$$

A expansão dos últimos termos em série de Taylor fornece:

$$
\ln \frac{\zeta-\zeta_{j+1}}{\zeta-\zeta_{j}}=\sum_{m=1}^{\infty} \frac{1}{m}\left(\frac{\zeta_{j+1}}{\zeta}\right)^{m}-\sum_{m=1}^{\infty} \frac{1}{m}\left(\frac{\zeta_{j}}{\zeta}\right)^{m}=-\sum_{m=1}^{\infty} \frac{\zeta_{j+1}^{m}-\zeta_{j}^{m}}{m \zeta^{m}}
$$

Retornando-se Eq. A.20, tem-se:

$$
\underset{j}{\Omega}=-\frac{\gamma}{2 \pi i}\left(c_{j} \zeta^{2}+c_{j} \varsigma+\underset{j}{c_{0}}\right) \sum_{m=1}^{\infty} \frac{\zeta_{j+1}^{m}-\zeta_{j}^{m}}{m_{j} \zeta^{m}}+\frac{\gamma}{2 \pi i} p_{j}(\zeta)
$$

Escrevendo o primeiro termo da expressão como a soma de um polinômio de ordem um e o segundo como uma série que converge para zero quando $\zeta^{-1}=0$, Strack (1989) apresenta o seguinte resultado:

$$
\stackrel{b}{\Omega_{j}}=-\frac{\gamma}{2 \pi i}\left[c_{2}\left(\zeta_{j+1}-\zeta_{j}\right) \zeta+\frac{1}{2} c_{j}\left(\zeta_{j+1}^{2}-\zeta_{j}^{2}\right)+c_{j}\left(\zeta_{j+1}-\zeta_{j}\right)\right]+\frac{\gamma}{2 \pi i} p_{j}+\frac{\gamma}{2 \pi i} \sum_{m=0}^{\infty} b_{m} \zeta^{-1}
$$


onde $b_{m}=\frac{1}{m+2} c_{j}\left(\zeta_{j+1}^{m+2}-\zeta_{j}^{m+2}\right)-\frac{1}{m+1} c_{j}\left(\zeta_{j+1}^{m+1}-\zeta_{j}^{m+1}\right)-\frac{1}{m} c_{j}\left(\zeta_{j+1}^{m}-\zeta_{j}^{m}\right)$

Como o segundo termo na Eq. A.27 é nulo $\left(\zeta^{-1}=0\right)$ e o potencial também deve ser nulo $\left(\Omega \rightarrow \zeta^{-1}\right.$ quando $\left.\zeta \rightarrow \infty\right)$ o polinômio $p_{j}$ é dado pelo termo entre colchetes na Eq. A.27 com ordem $n-1, n=2$ (ver Eq. 2.3.15). Utilizando-se as definições Eq. A.22, tem-se:

$$
\underset{j}{a_{1}}=\underset{j}{c_{2}}\left(\zeta_{j+1}-\zeta_{j}\right)=-\frac{1}{2}\left(\xi_{j+1}-\xi_{j}\right)
$$

$\mathrm{e}$

$$
\begin{aligned}
& \underset{j}{a_{0}}=\underset{j}{\frac{1}{2}} c_{2}\left(\zeta_{j+1}^{2}-\zeta_{j}\right)+\underset{j}{c_{1}}\left(\zeta_{j+1}-\zeta_{j}\right) \\
& =-\frac{1}{4}\left(\xi_{j+1}^{2}-\xi_{j}^{2}\right)-\frac{i}{4}\left(\xi_{j+1} \eta_{j+1}-\xi_{j} \eta_{j}+3 \xi_{j} \eta_{j+1}-3 \xi_{j+1} \eta_{j}\right)
\end{aligned}
$$

Substituindo-se $\underset{j}{a_{1}}$ e $a_{j}$ no polinômio $p_{j}$ na expressão Eq. A.26, o somatório no primeiro termo de Eq. 3.2.61 produz:

$$
\sum_{j=1}^{n} a_{1}=\frac{1}{2} \sum_{j=1}^{n}\left(\xi_{j+1}-\xi_{j}\right)=0
$$

$$
\text { e }
$$

$$
\sum_{j=1}^{n} a_{0}=-\frac{1}{4} \sum_{j=1}^{n}\left[\left(\xi_{j+1}^{2}-\xi_{j}^{2}\right)-i\left(\xi_{j+1} \eta_{j+1}-\xi_{j} \eta_{j}+3\left(\xi_{j} \eta_{j+1}-\xi_{j+1} \eta_{j}\right)\right)\right]
$$

Duas propriedades podem ser extraídas da expressão acima: a somatória do primeiro termo entre parênteses é nula e a somatória do último termo entre parênteses dividida por dois fornece a área da poligonal. Assim: 


$$
\stackrel{b}{\Omega}=\frac{\gamma}{2 \pi i} \sum_{j=1}^{n}\left(c_{j}^{2} \zeta^{2}+\underset{j}{1} \zeta+\underset{j}{c}\right) \ln \frac{\zeta-\zeta_{j+1}}{\zeta-\zeta_{j-1}}+\frac{\gamma A}{2 \pi} \ln \left(z-z_{1}\right)-\frac{3 \gamma A}{4 \pi}
$$

Para $\stackrel{a}{\Phi}$, é arbitrada a seguinte função de posição:

$$
\stackrel{a}{\Phi}=\frac{\gamma}{4 \pi i} \xi^{2} \sum_{j=1}^{n} \ln \frac{\varsigma-\varsigma_{j+1}}{\varsigma-\varsigma_{j-1}}
$$

Por fim, somando-se $\stackrel{a}{\Phi}$ a $\stackrel{b}{\Phi}$, tem-se a expressão completa do areasink:

$$
\stackrel{b}{\Omega}=\frac{\gamma}{2 \pi i} \sum_{j=1}^{n}\left(c_{j}^{2} \zeta^{2}+\underset{j}{1} \zeta+{ }_{j}^{0}+\frac{1}{2} \xi^{2}\right) \ln \frac{\zeta-\zeta_{j+1}}{\zeta-\zeta_{j-1}}+\frac{\gamma A}{2 \pi} \ln \left(z-z_{1}\right)-\frac{3 \gamma A}{4 \pi}
$$

\section{Referencias do Apêndice A}

Dagan, G., Fiori, A., Janković, I (2003) Flow and transport in highly heterogeneous formations: 1. Conceptual framework and validity of first-order approximations. Water Resour. Res. v39, n9, doi:10.1029/2002WR001717.

Fiori, A., Janković, I., Dagan, G. (2003) Flow and transport in highly heterogeneous formations: 2. Semianalytical results for isotropic media. Water Resour. Res. v39 n9. doi:10.1029/2002WR001719.

Janković, I. (1997) High Order Analytic Elements in Modeling Groundwater Flow. Tese de Doutorado. Universidade de Minnesota.

Janković, I., Fiori, A., Dagan, G. (2003) Flow and transport in highly heterogeneous formations: 3. Numerical simulations and comparison with theoretical results. Water Resour. Res. V39 N9. doi:10.1029/2002WR001721.

Strack, O. D. L. (1981) Flow in aquifers with clay laminae: 2. Exact solutions. Water Resour. Res. 17 (4), 993-1004.

Strack, O. D. L. (1989) Groundwater mechanics, Prentice-Hall, Englewood Cliffs. 


\section{Apêndice B}

\section{METODOLOGIA DE ZAADNOORDIJK PARA DETERMINAÇÃO DO POTENCIAL REAL PARA ELEMENTOS TRANSIENTES}

A metodologia apresentada por Zaadnoordijk (1988) para a formulação de elementos de escoamento transiente consiste de integrar soluções fundamentais, "He", da equação de calor (e.g. Carslaw e Jaeger, 1959) utilizando distribuições de densidade de esforços, “ $\lambda$ ”, na forma:

$$
\Phi=\int_{0}^{t} \int_{\zeta}^{t} \lambda \operatorname{Hed} \zeta d \tau
$$

Assim, é importante notar que a escolha da distribuição $\lambda$ desempenha um papel fundamental na formulação do elemento. As distribuições de esforços em Zaadnoordijk (1988) são feitas uniformemente e por polinômios de primeira ordem no espaço $\left(\zeta=\left.\xi\right|_{\xi} ^{\xi}\right)$ e no tempo $(\tau)$. A seguir, o elemento linesink é deduzido a título de ilustração da aplicação da metodologia de Zaadnoordijk para uma distribuição espacial de esforços uniforme e arbitrária no tempo.

Integrando uma distribuição uniforme de $\lambda$ em $\left.\xi\right|_{\xi} ^{\frac{2}{\xi}}$ como na equação Eq. 3.3.14, podese generalizar uma evolução polinomial de esforços fazendo $\lambda=\sigma_{n} \tau^{n}$ fazendo:

$$
\Phi=\int_{l s n}^{t}-\frac{\sigma \tau^{n} \sqrt{\alpha}}{4 \sqrt{\pi(t-\tau)}} e^{-\frac{\eta^{2}}{4 \alpha(t-\tau)}}\left\{\operatorname{erfc}\left(\frac{\xi-\stackrel{2}{\xi}^{-}}{2 \sqrt{\alpha(t-\tau)}}\right)-\operatorname{erfc}\left(\frac{\xi-\xi}{2 \sqrt{\alpha(t-\tau)}}\right)\right\} d \tau \quad \text { Eq. B.2 }
$$

Expandindo $\tau^{n}$ na forma binomial:

$$
\tau^{n}=\left\{t-(t-\tau)^{n}\right\}=\sum_{k=0}^{n}\left\{\left(\begin{array}{l}
n \\
k
\end{array}\right)(-1)^{k} t^{n-k}(t-\tau)^{k}\right\}
$$


onde $\left(\begin{array}{l}n \\ k\end{array}\right)=\frac{n !}{k !(n-k) !}$, qualquer expressão utilizada em elementos linesink será dada por uma somatória de integrais na forma:

$$
\underset{l s n}{\Phi}=\sum_{k=0}^{n}\left[\frac{\sigma\left(\begin{array}{l}
n \\
n
\end{array}\right)(-1)^{k+1} \tau^{n-k} \sqrt{\alpha}}{4 \sqrt{\pi}} \int_{0}^{t}(t-\tau)^{k-\frac{1}{2}} e^{-\frac{\eta^{2}}{4 \alpha(t-\tau)}}\left\{\operatorname{erfc}\left(\frac{\xi-\stackrel{2}{\xi}^{2}}{2 \sqrt{\alpha(t-\tau)}}\right) \operatorname{erfc}\left(\frac{\xi-\stackrel{1}{\xi}^{2}}{2 \sqrt{\alpha(t-\tau)}}\right)\right] d \tau\right]
$$

Eq. B. 4

As integrais acima são resolvidas por partes fazendo a seguinte mudança de variáveis, assim como em Theis (1935, Zaadnoordijk, 1988):

$$
u=\sqrt{\frac{\eta^{2}}{4 \alpha(t-\tau)}}, t-\tau=\frac{\eta^{2}}{4 \alpha u^{2}}, d \tau=\frac{\eta^{2}}{4 \alpha u^{3}} d u
$$

Utilizando-se ainda a definição:

$$
\stackrel{k}{I}_{1}=\int_{\sqrt{\frac{\eta^{2}}{4 \alpha t}}}^{\infty} \frac{1}{u^{2 k+2}} e^{-u^{2}}\left\{\operatorname{erfc}\left(u \frac{\xi-\stackrel{2}{\xi}}{\eta}\right)-\operatorname{erfc}\left(u \frac{\xi-\stackrel{1}{\xi}}{\eta}\right)\right\} d u \quad \text { para } k \geq 0
$$

o potencial devido a uma linesink torna-se expresso por:

$$
\Phi=2\left(\frac{\eta^{2}}{4 \alpha}\right)^{k+\frac{1}{2}} \stackrel{k}{I_{1}}
$$

Aplicando-se integração por partes à equação Eq. B.7 tem-se, para $k \geq 0$ : 


$$
\begin{aligned}
& I_{1}=\int_{\sqrt{\frac{\eta^{2}}{4 \alpha t}}}^{\infty} \frac{1}{2 k+1}\left(\sqrt{\frac{4 \alpha t}{\eta^{2}}}\right)^{2 k+1} e^{-\frac{\eta^{2}}{4 \alpha t}}\left\{\operatorname{erfc}\left(\frac{\xi-\xi}{\eta} \sqrt{\frac{\eta^{2}}{4 \alpha t}}\right)-\operatorname{erfc}\left(\frac{\xi-\xi}{\eta} \sqrt{\frac{\eta^{2}}{4 \alpha t}}\right)\right\}+ \\
& -\frac{1}{2 k+1} \frac{2}{\sqrt{\pi}} \int_{\sqrt{\frac{\eta^{2}}{4 \alpha t}}}^{\infty} \frac{1}{u^{2 k+1}}\left\{\frac{\xi-\xi^{2}}{\eta} e^{-u^{2} \frac{\left(\xi-\xi^{2}\right)^{2}+\eta^{2}}{\eta^{2}}}-\frac{\xi-\xi}{\eta} e^{-u^{2} \frac{(\xi-\xi)^{2}+\eta^{2}}{\eta^{2}}}\right\} d u+ \\
& -\frac{2}{2 k+1} \int_{\sqrt{\frac{\eta^{2}}{4 \alpha t}} \frac{1}{u^{2 k}}}^{\infty} e^{-u^{2}}\left\{\operatorname{erfc}\left(u \frac{\xi-\frac{2}{\xi}}{\eta}\right)-\operatorname{erfc}\left(u \frac{\xi-\xi}{\eta}\right)\right\} d u
\end{aligned}
$$

Atentando-se para o último termo da expressão acima, observa-se uma relação recursiva na definição Eq. B.7 e o segundo termo da expressão aparece como uma integral da qual será definida uma função referida por $I_{2}^{k}$ como:

$$
I_{j}^{k}=\int_{\sqrt{\frac{\eta^{2}}{4 \alpha t}}}^{\infty} \frac{1}{u^{2 k+1}} e^{-u^{2} \frac{(\xi-\xi)^{2}+\eta^{2}}{\eta^{2}}} d u \quad j=1,2 \quad k \geq 0
$$

O último termo da expressão Eq. B.8 é então escrito como $I_{1}^{k-1}$ e a solução de Eq. B.7 é reescrita para $k \geq 0$ como:

$$
\begin{aligned}
& \stackrel{k}{I}_{1}=\int_{\sqrt{\frac{\eta^{2}}{4 \alpha t}}}^{\infty} \frac{1}{2 k+1}\left(\sqrt{\frac{4 \alpha t}{\eta^{2}}}\right)^{2 k+1} e^{-\frac{\eta^{2}}{4 \alpha t}}\left\{\operatorname{erfc}\left(\frac{\xi-\stackrel{2}{\xi}^{2}}{\eta} \sqrt{\frac{\eta^{2}}{4 \alpha t}}\right)-\operatorname{erfc}\left(\frac{\xi-\frac{1}{\xi}}{\eta} \sqrt{\frac{\eta^{2}}{4 \alpha t}}\right)\right\}+ \\
& -\frac{1}{2 k+1} \frac{2}{\sqrt{\pi}}\left\{\frac{\xi-\xi_{\xi}^{k}}{I_{2}}-\frac{\xi-\stackrel{1}{\xi}_{2}^{k}}{\eta} I_{2}\right\}-\frac{2}{2 k+1} \stackrel{k-1}{I}_{1}
\end{aligned}
$$

A solução da integral $I_{2}^{k}$ é da mesma forma obtida aplicando-se integração por partes, obtendo-se para $\mathrm{k}>0$ : 


$$
\underset{j}{I_{2}}=\frac{1}{2 k}\left(\frac{4 \alpha t}{\eta^{2}}\right)^{k} e^{-\frac{(\xi-\xi)^{2}+\eta^{2}}{\eta^{2}}}-\frac{1}{k} \frac{(\xi-\xi)^{2}+\eta^{2}}{\eta^{2}} \int_{\sqrt{\frac{\eta^{2}}{4 \alpha t}}}^{\infty} \frac{1}{u^{2 k-1}} e^{-u^{2} \frac{(\xi-\xi)^{2}+\eta^{2}}{\eta^{2}}} d u \quad j=1,2 \text { Eq. B. } 11
$$

Da mesma forma que na equação Eq. B.7, a solução de $I_{2}^{k}$ exibe uma relação de recursividade que pode ser expressa como:

$$
\underset{j}{I_{2}}=\frac{1}{2 k}\left(\frac{4 \alpha t}{\eta^{2}}\right)^{k} e^{-\frac{(\xi-\xi)^{2}+\eta^{2}}{\eta^{2}}}-\frac{1}{k} \frac{\left(\xi-\xi^{j}\right)^{2}+\eta^{2}}{\eta^{2}}{\underset{j}{j}}_{j}^{k-1} \quad \mathrm{j}=1,2 \quad \mathrm{k}>0
$$

Determinando-se então as expressões para ambas integrais $\stackrel{k}{I_{1}}$ e $\stackrel{k}{I_{2}}$ para $k=0$, tem-se:

$$
\begin{aligned}
& I_{1}=\sqrt{\frac{4 \alpha t}{\eta^{2}}} e^{-\frac{\eta^{2}}{4 \alpha t}}\left\{\operatorname{erfc}\left(\frac{\xi-\xi^{2}}{\eta} \sqrt{\frac{\eta^{2}}{4 \alpha t}}\right)-\operatorname{erfc}\left(\frac{\xi-\xi}{\eta} \sqrt{\frac{\eta^{2}}{4 \alpha t}}\right)\right\}+ \\
& -\frac{2}{\sqrt{\pi}} \int_{\sqrt{\frac{\eta^{2}}{4 \alpha t}}}^{\infty} \frac{1}{u}\left\{\frac{\xi-\xi}{\eta} e^{-u^{2} \frac{(\xi-\xi)^{2}+\eta^{2}}{\eta^{2}}}-\frac{\xi-\xi}{\eta} e^{-u^{2} \frac{(\xi-\xi)^{2}+\eta^{2}}{\eta^{2}}}\right\} d u+ \\
& -2 \int_{\sqrt{\frac{\eta^{2}}{4 \alpha t}}}^{\infty} e^{-u^{2}}\left\{\operatorname{erfc}\left(u \frac{\xi-\stackrel{2}{\xi}^{2}}{\eta}\right)-\operatorname{erfc}\left(u \frac{\xi-\xi}{\eta}\right)\right\} d u
\end{aligned}
$$

onde o segundo termo é a "função poço" E1 escrita na forma:

$$
\int_{\sqrt{\frac{\eta^{2}}{4 \alpha t}}}^{\infty} \frac{1}{u} e^{-u^{2} \frac{(\xi-\xi)^{2}+\eta^{2}}{\eta^{2}}} d u=\left.\mathrm{E} 1(w)\right|_{w=\frac{(\xi-\xi)^{2}+\eta^{2}}{4 \alpha t}}
$$

Assim, tem-se: 


$$
\begin{aligned}
& \stackrel{0}{I}_{1}=\sqrt{\frac{4 \alpha t}{\eta^{2}}} e^{-\frac{\eta^{2}}{4 \alpha t}}\left\{\operatorname{erfc}\left(\frac{\xi-\xi}{\eta} \sqrt{\frac{\eta^{2}}{4 \alpha t}}\right)-\operatorname{erfc}\left(\frac{\xi-\xi}{\eta} \sqrt{\frac{\eta^{2}}{4 \alpha t}}\right)\right\}+ \\
& -\frac{1}{\sqrt{\pi}}\left\{\frac{\xi-\stackrel{2}{\xi}^{\prime}}{\eta} \mathrm{E} 1\left(\frac{\left(\xi-\stackrel{2}{\xi}^{2}+\eta^{2}\right.}{4 \alpha t}\right)-\frac{\xi-\stackrel{1}{\xi}^{\prime}}{\eta} \mathrm{E} 1\left(\frac{\left(\xi-\stackrel{1}{\xi}^{2}+\eta^{2}\right.}{4 \alpha t}\right)\right\}+\quad k \geq 0 \quad \text { Eq. B.15 } \\
& -2 \int_{\sqrt{\frac{\eta^{2}}{4 \alpha t}}}^{\infty} e^{-u^{2}}\left\{\operatorname{erfc}\left(u \frac{\xi-\stackrel{2}{\xi}^{2}}{\eta}\right)-\operatorname{erfc}\left(u \frac{\xi-\xi}{\eta}\right)\right\} d u
\end{aligned}
$$

Utilizando-se agora a função E1 na expressão Eq. B.11, pode-se escrever:

$$
\underset{j}{I_{2}}=\frac{1}{2} \mathrm{E} 1\left(\frac{\left(\xi-{\stackrel{j}{\xi})^{2}+\eta^{2}}^{2}\right.}{4 \alpha t}\right) \quad \mathrm{j}=1,2 \quad k=0
$$

Assim, as integrais $\stackrel{k}{I_{1}}$ e $\stackrel{k}{I}_{2}$ para $k>0$ podem ser obtidas substituindo-se termos de índices $k$ inferiores recursivamente até o $k$ desejado. 


\section{Apêndice C}

\section{MÉTODO DE IMAGENS PARA CONTORNOS DO TERCEIRO TIPO}

\section{C.1Fórmula de Keller}

Segundo Keller (1953) o método de imagens pode ser aplicado a contornos de condição mista (terceiro tipo), onde é especificado que:

$$
\partial \mathrm{G} / \partial \eta+\lambda \mathrm{G}=0
$$

onde $\lambda$ é uma constante definida para cada fronteira onde a condição é especificada. Quando tal condição é imposta, é necessário obter a expressão de "G" que satisfaz a equação Eq. C.1 para $n$ dimensões consideradas, definindo o operador reflexão para este caso. Para o caso do semi-plano horizontal $x \geq 0$, a função $\mathrm{G}$ é dada pela seguinte expressão:

$$
G\left(x, x^{\prime}\right)=G^{*}\left(x, x^{\prime}\right)+G^{*}\left(-x, x^{\prime}\right)+2 \lambda \int_{-\infty}^{-x} e^{\lambda(x+u)} G^{*}\left(u, x^{\prime}\right) d u
$$

onde assume-se que $\mathrm{G}^{*}$ é uma função par em relação a $x^{\prime}=0$. Observe que o terceiro termo da expressão inclui infinitas imagens infinitesimalmente próximas de forma assimétrica sobre o semi-plano $-x \leq 0$, cujos parâmetros de esforços são dados por $2 \lambda e^{\lambda(x+u)}$. Assim, pode-se verificar que a função Eq. C.2 satisfaz a expressão Eq. 4.1.1, já que o terceiro termo da expressão também define uma função de Green. A definição de condições de contorno por meio da sobreposição de efeitos simétricos a cada fronteira é algo intuitivo. A inclusão de um termo assimétrico ao somatório Eq. 4.1.1, por outro lado, torna-se então algo que vem contra essa intuição. No entanto, permanece a definição do método segundo Keller (1953) de que uma função de Green pode ser obtida a partir da especificação de condições de contorno por meio da sobreposição de funções de Green primitivas. Isto posto, Keller (1953) particulariza a condição dos ângulos entre planos $(\alpha)$ para que nenhuma imagem caia em $D$ (Eq. C.3) e reescreve Eq. 4.1.1 como: 


$$
\begin{aligned}
& \alpha=\pi / p, p=1,2 \\
& \mathrm{G}\left(r, r^{\prime}\right)=\sum_{r^{\prime \prime} \in S(r)} A\left(r^{\prime \prime}\right) \mathrm{G}^{*}\left(r^{\prime \prime}, r^{\prime}\right)
\end{aligned}
$$

onde $A$ é o parâmetro de esforço da função $\mathrm{G}^{*}\left(r^{\prime \prime}, r^{\prime}\right)$.

\section{C.2Fórmula de Anderson}

Diversos autores (e.g. Bear, 1972, pág. 312 e Polubarinova-Kochina, 1962, pág 372) mostram ainda aplicações onde o método de imagens é aplicado em problemas de sobreposição de poços sujeitos a fronteiras entre formações de diferentes condutividades. Dessa forma, especifica-se ambas condições, Dirichlet e Neuman, simultaneamente sobre a fronteira. As expressões que definem a reflexão dos poços neste caso são obtidas impondo-se o posicionamento do poço e sua imagem e a expressão Eq. 3.4.15 no contorno. Assim, seguindo a simbologia definida na expressão Eq. 4.3.5, tem-se:

$$
\begin{aligned}
& \stackrel{\text { IeII }}{\Theta}=1+2 \beta \quad \text { para } \eta^{+} \\
& \stackrel{\text { IeII }}{\Theta}=(1-2 \beta) \text { para } \eta^{-},
\end{aligned}
$$

onde $\beta=\left[\frac{K^{+}-K^{-}}{K^{+}+K^{-}}\right]$. A expressão Eq. C.5 é válida para o domínio à frente do "espelho" e a expressão Eq. C.6 para o domínio atrás do "espelho" e invertendo-se o posicionamento de $\stackrel{p o s o, e}{\lambda}$ e $\stackrel{\text { poço }}{\lambda}$ na expressão Eq. 4.3.5 para pontos localizados atrás do "espelho".

Anderson (2000) apresenta uma abordagem possível para contornos do terceiro tipo. No artigo é abordado o problema do escoamento no plano vertical de um aqüífero sobre uma base impermeável e semiconfinado por uma camada drenante fina e pouco permeável o suficiente para que o escoamento nela tenha somente uma componente na direção vertical. A camada drenante representa, portanto, o contorno do topo do domínio e a base, por sua vez, o contorno inferior. Na abordagem de Anderson (2000), a especificação do contorno superior é feita, em um primeiro momento, considerando-se uma espessura $H^{*}$ e uma condutividade $K^{*}$ 
para a formação superior. No segundo momento, a espessura e a condutividade da formação superior são levadas ao limite tendendo a zero, de maneira que a camada passa a ser considerada como uma lente de condutância $c=H^{*} / K^{*}$.

Dessa forma, o modelo é concebido, no primeiro momento, por duas faixas dominiais: o aqüífero sobre a base impermeável e a formação superior cujo topo apresenta um único valor de carga piezométrica. O método de imagens é aplicado então a ambas as faixas de domínio. A fronteira entre as formações é especificada pelas expressões Eq. C.5 e Eq. C.6. A expressão Eq. 4.3.11 é empregada na fronteira que define a base do aqǘfero e a expressão Eq. 4.3.6 no topo.

Obtida a expressão para o modelo de imagens para a primeira abordagem, o resultado é levado ao limite de $H^{*}, K^{*} \rightarrow 0$ produzindo-se (Anderson 2000, Eq. 27):

$$
\Omega=\frac{Q}{2 \pi} \ln \left(z-z_{p}\right)\left(z-\overline{z_{p}}\right)+\frac{Q}{\pi} \frac{i}{c K} \int_{\frac{z_{p}}{z_{p}}+i \infty} e^{\frac{i}{c K}\left(\delta-\overline{z_{p}}\right)} \ln (z-\delta) d \delta
$$

Note-se que a expressão obtida por Anderson (2000) é idêntica à expressão declarada por Keller (1953) para a representação de contornos do terceiro tipo em termos de sobreposições (imagens) de funções de Green, $G^{*}\left(x, x^{\prime}\right)=\ln \left(z-z_{p}\right)$, (cf. Eq. C.2, fazendo $\lambda=\frac{i}{c K},-x=\overline{z_{p}}, x=z_{p}, x^{\prime}=z-\delta$ e $\left.u=\delta\right)$.

Após algebrismos, a expressão Eq. C.7 pode ser reescrita de forma que o terceiro termo apresente uma linha dipolo semi-infinita estendida a partir do poço imagem até o infinito na direção da projeção sobre o "espelho" com esforço de sinal negativo (ver Anderson, 2000, pág. 466). Assim, a expressão Eq. C.7 passa a ser dada para o caso de um poço por:

$$
\Omega=\frac{Q}{2 \pi} \frac{\ln \left(z-z_{p}\right)}{\ln \left(z-\overline{z_{p}}\right)}-\frac{Q}{\pi} \exp \left[\frac{i}{c K}\left(z-\overline{z_{p}}\right)\right] \mathrm{E} 1\left[\frac{i}{c K}\left(z-\overline{z_{p}}\right)\right]
$$

Observe que o primeiro termo da expressão apresenta a soma do efeito de um poço real e o poço que é sua imagem invertida. O segundo termo representa um dipolo semi-infinito originado em $z=\overline{z_{p}}$ com o módulo do esforço exponencialmente decrescente.

A representação obtida por Anderson (2000), por sua vez, para a imagem de uma linha dipolo reproduz a mesma estrutura da expressão Eq. C.8 onde o último termo é dado 
novamente por uma linha dipolo estendida na direção oposta à projeção sobre o espelho. Uma vez observado que o último termo é comum para poços e linhas dipolos, as imagens de elementos dados por funções derivadas de ambas exibem também a mesma estrutura apresentada por Anderson (2000, Eq. 39):

$$
\stackrel{e l}{\Omega}=\stackrel{e l}{\lambda}_{(j)} \stackrel{e l}{\Lambda}_{(j)}\left(z_{p}\right)-\stackrel{e l}{\lambda}_{(j)} \stackrel{e l}{\Lambda}_{(j)}\left(\overline{z_{p}}\right)-\frac{\mu}{\pi} \exp \left[\frac{i}{c K}\left(z-\overline{z_{p}}\right)\right] \mathrm{E} 1\left[\frac{i}{c K}\left(z-\overline{z_{p}}\right)\right] \quad \text { Eq. C.9 }
$$

onde o parâmetro $\mu$ generaliza a vazão $Q$ definida para o caso de poços (Eq. C.7) e os termos $\stackrel{e l}{\lambda}_{(j)} \stackrel{e l}{(j)}_{(}$podem conter elementos analíticos apresentados no capítulo 3 . No processo de construção de imagens de imagens, o termo $\stackrel{e l}{\lambda}_{(j)}^{e l} \Lambda_{(j)}$ também inclui a linha dipolo semiinfinita. Pode-se, então, concluir da expressão Eq. C.9, que o método de imagens pode ser utilizado em um modelo de elementos analíticos para a especificação de condições de contorno do terceiro tipo.

Observa-se, no entanto, que no modelo da expressão Eq. C.9 são introduzidos dois novos parâmetros, diferentemente das demais aplicações do método de imagens, onde apenas um parâmetro do elemento imagem está associado a cada parâmetro do elemento original. Observa-se, ainda, que os parâmetros $j$ do elemento imagem $\left.\stackrel{e l}{\left(\lambda_{(j)}\right.} \stackrel{e l}{\Lambda} \Lambda_{(j)}\left(\overline{z_{p}}\right)\right)$ estão relacionados com os parâmetros do elemento original por um sinal oposto, como acontece na especificação de contornos do primeiro tipo para poços (ver Eq. 4.3.6). É necessário, portanto, especificar uma expressão para o parâmetro $\mu$ de forma que seja possível obter a solução dos parâmetros de todos os elementos do modelo.

O esforço sobre a linha dipolo é definido por $\frac{\mu}{\pi} \exp \left[\frac{i}{c K}\left(z-\overline{z_{p}}\right)\right]$ e define o ramo das linhas de corrente ao longo do dipolo (ver seção 2.3.2). Pode-se observar que a linha dipolo adicionada à imagem do elemento em Eq. C.9 define o decaimento do ramo em relação ao valor $\mu$ em $z=\overline{z_{p}}$, necessário para obter a especificação da condição do terceiro tipo no contorno. Assumindo um valor constante para $\mu$, o ramo do elemento original é refletido para trás do "espelho" e, então, é especificada a condição de primeiro tipo sobre o contorno. Observe que na função Eq. C.8, o valor de $-\mu$ é igual a $-Q$, que por sua vez define o ramo de um poço imagem (ver seção 2.2.3). Assim, o parâmetro $\mu$ poderá ser especificado pelo valor do ramo do elemento imagem. 


\section{Referências do Apêndice C}

Steward, D. R. (2001). A vector potential for a partly penetrating well and flux in an approximate method of images. Proceedings of The Royal Society of London, Series A 457(2013), 2093-2111.

Bakker, M. e Anderson, E. I. (2004) Steady Flow to a Well Near a Stream with a Leaky Bed. Ground Water. v41, n6, pp833-840.

Luther, K. e Haitjema, H.M. (2000) Approximate analytic solutions to 3D unconfined groundwater flow within regional 2D models, Journal of Hydrology, n. 229, pp. 101-117.

Anderson, E. I. (2000) The method of images for leaky boundaries. Adv. Water Resour., 23,461-74.

Keller, J.B. (1953) The scope of the Image Method, Comm. on Phys. and App. Math. v4, pp505-512. 


\section{Índice de Autores}

Casos

$\begin{array}{ll}\text { Anderson } & 141,203,204 \\ \text { Bakker } & 58,107,108,141 \\ \text { Barnes } & 83,88,92,119,120 \\ \text { Batista } & 154,160,171,177 \\ \text { Bear } & 21,22,23,28,29,30,31,32,33,34,43,53,203 \\ \text { Bischoff } & 154 \\ \text { Carslaw } & 44,80,82,90,122,138,197 \\ \text { Castro } & 15,160,169,170,171 \\ \text { Dagan } & 189 \\ \text { Feitosa } & 169,175,177 \\ \text { Fetter } & 17 \\ \text { FGV } & 160 \\ \text { Fiori } & 189 \\ \text { Furman } & 79 \\ \text { Georghitza } & 26 \\ \text { Haitjema } & 61,106,129 \\ \text { Hidroservice } & 159,160,161,162,164,166,172,173 \\ \text { Jaeger } & 44,80,82,90,122,138,197 \\ \text { Keller } & 129,130,131,132,139,154,202,204 \\ \text { Kobe } & 34 \\ \text { Manuel Filho } & 15,160,169,170,171 \\ \text { Mattos } & 17 \\ \text { Melo } & 169,175,177 \\ \text { Mitchell-Bruker } & 106 \\ \text { Muskhelishvili } & 44 \\ \text { Neuman } & 79 \\ \text { Secrest } & 145,146 \\ \text { Steward } & 129 \\ \text { Strack } & 15,31,35,36,40,42,44,46,47,48,56,58,59,61,64,66,69,70,72, \\ \quad 73,75,78,79, & 80,81,83,88,92,100,102,105,106,108,119,120,121,122,124,125, \\ \quad 126,127,129,132,157,189,193,194 \\ \text { Stroud } & 145,146 \\ \text { Villela } & 17 \\ \text { Zaadnoordijk } & 58,78,80,81,82,83,85,87,88,90,91,92,94,95,96,97,98,119,121, \\ \quad 122,124,125,126,127,157,197,198 \\ \end{array}$




\section{Índice Remissivo}

Água Subterrânea, 17

Aqüífero

Barreiras, 7, 15, 159, 160, 172, 174, 184

aqüíferos, 17, 18

aluvionares, 137

anisotrópicos, 20, 31

bordas dos, 128

confinados, 22, 24, 26, 50, 59, 60, 78, 104,106

costeiros, 137

heterogêneos, 21, 31

homogêneos, 24

homogêneos, 25

infinitos, 147

isotrópicos, 20, 25

livres, 22, 59, 60, 78, 104, 106, 115

semiconfinados, 25

Aqüíferos

Longos, 7, 15, 16, 128, 137, 159, 183

areasink, 72, 77, 78, 95, 96, 98

balanço hídrico, 62, 63, 65, 66, 67, 89, 112

campo

conservativo, 28

de escoamento, 21, 27, 28, 30, 32, 34, 35,115

gravitacional, 27

irrotacional, 29, 34

rotacional, 31,34

solenóide, 29, 32

campos

de escoamento, 59 campos vetoriais, 34,37

Cauchy-Riemann, 31, 36, 40, 41

ciclo de vida, 108

classe, 108

coeficiente de armazenamento, 22

coeficiente de armazenamento específico, 21

complexas

coordenadas c., $35,39,45$

funções c., 36, 37, 55

Funções c., 36

variáveis c., 38,44

condição de contorno, 113

condições de contorno

condições transientes, 106

diferentes transmissividades, 103

dreno ideal, 102

especificando carga, 100

impermeável, 137

leitos colmatados, 106

objetos drenantes, 100

obstáculos semi-impermeáveis, 105

paredes impermeáveis, 102

primeiro tipo, 100, 131, 134, 136, 139

segundo tipo, 100, 102, 103, 131, 134,

136,140

terceiro tipo, 105, 141

condutividade hidráulica, 19, 22, 28

Darcy, 19

decomposição de Helmholtz

ver teorema de Helmholtz, 34 
densidade de esforços, 61, 82, 83, 87, 93, 98

distribuição de esforços, 62, Consulte

elementos analíticos, 16, 46, 58, 99, 107, 128,138

elementos estacionários, 61,110

elementos transientes, 115

Equação

da Continuidade, 21, 23, 27, 29, 31

de Boussinesq, 23, 78

de Helmholtz, 25, 79

de Laplace, 24, 29, 37, 43, 72, 129, 132

de Poisson, 24, 32, 37, 72, 73, 75, 137 ,

138

do Calor, 24, 81, 138, 139

escoamento

horizontal, 38

regional, 61, 63, 78, 79

rotacional, 41

superficial, 18

transiente, 23

uniforme, 37, 38, 40, 114

escoamento de águas subterrâneas

ver escoamentos subterrâneos, 21, 27

escoamento de interface

ver escoamentos multifásicos, 52

escoamento em meios porosos

ver escoamentos subterrâneos, 30

escoamento não-permanente

ver escoamentos transientes, 138

escoamentos

estacionários, 23

multifásicos, 42 subterrâneos, 22, 25, 34, 37, 44, 51, 52, $59,99,128$

transientes, 79, 106, 153, 197

escoamentos em meios porosos

ver escoamentos subterrâneos, 16, 27, 28

Fraturas

ver objetos drenantes, 100

fronteiras

externas, 128, 129, 138, 141, 147, 148

internas, 138

fronteiras externas, 53

função

de Green, 129, 131

de poço, 61, 62

de Zhukovski, 43

dipolo, 40, 47, 61, 62, 63, 80, 189

dipolo instantâneo, 92

linha poço, 47

poço, 80,200

vórtice, 41,42

funções

de influência, 62, 66, 82, 90, 99, 106, $107,114,153$

de influência, 63

harmônicas, 37, 42, 62, 139

grau

um, 96, 97, 98

zero, 89, 91, 95

heranças, 108

instanciação, 108

integrais complexas

ver integrais de Cauchy, 44

integrais de Cauchy 
ver integrais singulares, 44

integrais singulares, 44

Lagoa

do Bonfim, 159, 160, 163, 165, 167, $169,170,171,175,177,179$

lei de Darcy, 20, 23, 27, 28, 30, 59, 60

linedipoles, 101, 102

linedoublet, 67, 69, 73, 76, 90, 95, 96, 97, 104

linesink, 64, 65, 73, 77, 82, 83, 85, 86, 87, $89,90,96,100$

linguagem de programação, 107

linguagens orientadas com objetos, 108

linha de corrente, 30, 31, 32, 38, 41

mapeamento

de Schwarz-Christoffel, 54

de Zhukovski, 43

matriz

cheia, 99

construção da, 113

de coeficientes, 99, 113, 114

solução da, 113

meio ambiente, 18

meio poroso, 20

Método de Imagens, 53, 54, 128, 129, 130,

$131,139,147,148,154$

modelo, 58

modelos realistas, 58

Navier-Stokes, 19

objetos drenantes

ver condições de contorno, 100

ordem

do elemento, 64

do polinômio, 82,90 dois, $64,65,69,89,95$

elevada, 61

primeira, 69, 97

segunda, 61, 69

um, 65, 82, 83, 85, 91, 96, 98

permeabilidade intrínseca, 19

polinômios de Chebyshev, 61

ponto de referência, 63, 99, 111

pontos de controle, 98

porosidade efetiva, 20

potencial

complexo, 36, 37, 38, 41, 45, 46, 64

de descarga, 28, 30, 59

de Newton, 42, 43, 51

de referência, 38, 63, 99, 111, 114

de Zhukovski, 44

logarítmico, 39

unificado, 60

potencial de referência, 110

princípio de Duhamel, 34

procedimento, 108

propriedade, 108

ramo, 39, 42, 46

recarga, 22, 25, 44, 62, 72, 75, 137, 138,

147,155

região de interesse, 128

regime transiente, 23

regra de Leibnitz, 59

Schwarz-Christoffel

transformações de, 128, 132, 147, 154

Sistema

Adutor Agreste/Trairi/Potengi, 15, 159, $161,165,179$ 
Lacustre Bonfim, 15, 159, 162, 163, $165,167,171$

sistemas multicamada, 25

sobre-especificação, 113

sobreposição de efeitos, 32, 54, 58

suposições de Dupuit, 22

teorema de Duhamel, 34

de Helmholtz

ver decomposição de Helmholtz, 34

de Stokes, 29

$\operatorname{Tim}^{\mathrm{SL}}, 107,112,114$

transformações de coordenadas, 108 